Academy of Sciences, Russian Federation Institute for Dynamics of the Geospheres

\title{
Generation of Low-Frequency Electric and Magnetic Fields During Large-Scale Chemical and Nuclear Explosions
}

V.V. Adushkin

Director of IDG RAS

V.A. Dubinya

V.A. Karaseva

S.P. Soloviev

V.V. Surkov 


\section{DISCLAIMER}

This report was prepared as an account of work sponsored by an agency of the United States Government. Neither the United States Government nor any agency Thereof, nor any of their employees, makes any warranty, express or implied, or assumes any legal liability or responsibility for the accuracy, completeness, or usefulness of any information, apparatus, product, or process disclosed, or represents that its use would not infringe privately owned rights. Reference herein to any specific commercial product, process, or service by trade name, trademark, manufacturer, or otherwise does not necessarily constitute or imply its endorsement, recommendation, or favoring by the United States Government or any agency thereof. The views and opinions of authors expressed herein do not necessarily state or reflect those of the United States Government or any agency thereof. 


\section{DISCLAIMER}

Portions of this document may be illegible in electronic image products. Images are produced from the best available original document. 


\section{DISCLAIMER}

This document was prepared as an account of work sponsored by an agency of the United States Government. Neither the United States Government nor the University of California nor any of their employees, makes any warranty, express or implied, or assumes any legal liability or responsibility for the accuracy, completeness, or usefulness of any information, apparatus, product, or process disclused, or represents that its use wuuld not infringe privately owned rights. Reference herein to any specific commercial products. process, or service by trade name, trademark, manufacturer, or otherwise, does not necessarily constitute or imply its endorsement, recommendation, or favoring by the United States Government or the University of California. The views and opinions of authors expressed herein do not necessarily state or reflect those of the United States Government or the University of California. and shall not be used for advertising or product endorsement purposes. 


\section{DISCLAIMER}

Portions of this document may be illegible in electronic image products. Images are produced from the best available original document. 


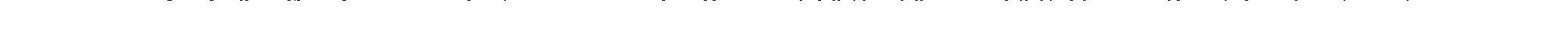




\section{INSTITUTE FOR DYNAMICS OF GEOSPHERES RUSSIAN ACADEMY OF SCIENCES \\ $* * *$ \\ UNIVERSITY OF CALIFORNIA \\ LAWRENCE IIVERMORE NATIONAL IABORATORY}

GENERATION OF LOW-FREQUENCY ELECTRIC AND MAGNETIC FIELDS DURING IAARGE-SCALE CHEMICAL AND NUCLEAR EXPLOSIONS

May 1995

Technical Report

CONTRACT No. B239748

Moscow-1995 
Project executors:

V.V.Adushkin

S.P.Soloviev

V.V.Surkov

V.A. Dubinya

V.A.Karaseva 
Contents

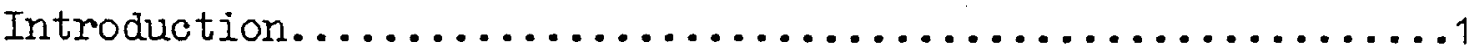

1. Natural low-frequency electromagnetic field of the Earth

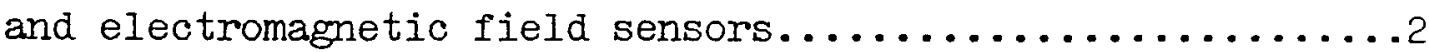

1.1. Electric processes in the surface layer of the

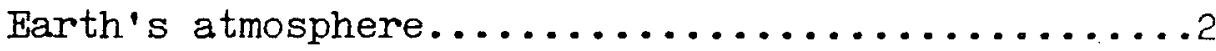

1.2. Measurement techniques of electric field in the surface layer of the atmosphere.............

1.3. Observations of the time variations of the electric

field in the surface layer of the atmosphere.....20

1.4.Disturbances of the atmospheric electric field

from the sources of man-made and natural origin...26

2. Electromagnetic signals generated by detonation of high

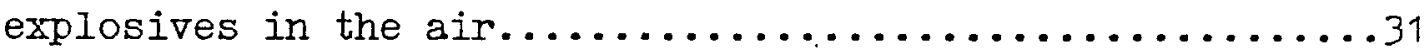

3. Electromagnetic signals generated by underground chemical

and nuclear explosions........................43

3.1. Electromagnetic emission generated by the nuclear explosions...............................44

3.2. Source modeling for low-frequency electromagnetic

signals in the soil........................ . 47 .

3.2.1.Effects of shock polarization and depolarization of solid dielectrics.................44

3.2.2. Flectric field in the growing crack and emission of the particles during the fragmentation of the matter........................64

3.2.3. Electromagnetic field of the shock wave during the underground explosion...............71

3.3. Electromagnetic signals generated by the seismic waves................................ 86

3.3.1. Instrumentation and experimental results........91

3.3.2.Equations of the motion of the elastic conductive medium in the magnetic field..............98

3.3.3. Approximate calculations of the seismoinductive effect of the underground explosions..........106

3.4.Disturbance of the atmosphere's electric field during the excavation explosions and underground

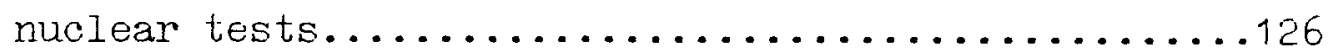


3.4.1. Shape of the electric field signals and the dependence of the signals amplitude on the explosion depth..........................127

3.4.2. Influence of the soil properties on the electric

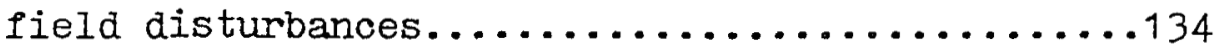

3.4.3. Dependence of the amplitude of the electric field signals from the distance to the

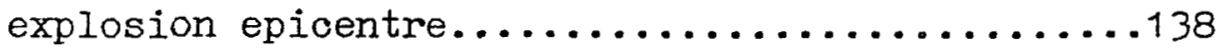

3.4.4. Influence of the explosive charge mass on the electric field disturbances..................147

3.4.5. Electrio field generated by the underground

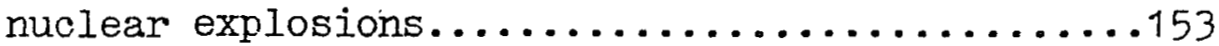

4.Iong-term anomalies of the earth's electromagnetic field in the vicinity of underground explosions...............159 4.1. Effects of the shock magnetization of heterogeneous media.................................159

4.2. Effects of the currents redistribution and change of the conductivity in fragmented media..............165 Conclusions....................................171 References........................................172 
Introduction

This report consists of four chapters. In the first chapter, we discuss the main parameters of the electric field in the surface layer of the atmosphere and the results of the investigations of the natural electric field variations. Moreover, the measurement techniques of the electric field and magnetic field variations are considered in this chapter.

The description of the experimental investigations of the electromagnetic field for the explosions in the air is represented in the second chapter.

The third chapter is concerned with the electromagnetic signals generated by underground nuclear and chemical explosions. The large-scale explosions that was monitored for electromagnetic signals from 1976 through 1991 are listed.

The effect of the shock polarization and its manifestation for the large-scale explosions considered there. The physical mechanism of the polarization of the heterogeneous medium at the shock wave front is conditioned by the formation of the dipole moments near the shear cracks and emptiness and inclusions, which are compressed asymmetrically. The effect of the shock polarization in the rock results in the arise of own electromagnetic field of the shock wave. On the surface of the weakly conducive medium in the nearest zone the field decreases in agreement with power law, and its value mainly depends on the horizontal component of the effective dipole, which is formed by the matter polarized in the shook wave. Formulas, which allow to determine the field magnitude at different distances in relation to the parameters of the medium and the shock wave, were founded too.

Furthermore, we considered the electromagnetic signals generated by the seismic waves after the underground explosions. These electromagnetic disturbances are connected with the motion of the conductive soil in the natural magnetic field. The relations between the values of the magnetic disturbances and the mass velocity in the wave were established here.

In the next chapter we consider the disturbances of the electric field in the surface layer of the atmosphere generated by the underground chemical and nuclear explosions. Our results indicates that explosions are capable of creating large electric charge separations in the atmosphere. A simple model of field generation is proposed. Furthermore, a correlation between the magnitude of the explosion and the electric charge of dust cloud is established.

Long-term anomalies of the Earth's electromagnetic field in the vioinity of underground explosions were investigated in the fourth chapter. Study of the phenomenon of the irreversible shock magnetization showed that in the zone nearest to the explosion the quasistatical magnetic field decreases in inverse proportion to the distance.

We considered the effect of the distortion of the local electromagnetic field by the currents, generated in the ground because of the electrochemical processes and contact potential difference on the surface of the up-hole emplacement pipe. Variations in conductivity of the medium in the vicinity of the underground cavity was analyzed too. We obtained the relation between the anomalies of local geomagnetic and geoelectric fields on the surface of the medium and the conductivity and dimensions of the destruction zone. 
1. Natural electromagnetic field of the Earth and methods of its registration.

1.1. Electric processes in the surface layers of the Earth's atmosphere.

Electric field in the atmosphere, as well as any electric field, in each point might be characterized by the value of the potential. This potential is caused by all electric charges, which are present on the Earth's surface and in the atmosphere. Knowledge about the potential distribution in the atmosphere allows to find electric field strength for each point (potential gradient with negative sign). Besides the electric field strength, the conductivity, spectrum of the atmospheric ions, space electric charges and currents are electric characteristics of the atmosphere. These characteristics of the atmospheric electric field have a local character and are connected with each other. So, electric charge density $\rho$ is connected with the eleotric field strength $E$ by the Poisson's equation, and current density $f$ is connected with $E$ by $\mathrm{Ohm}^{\prime} \mathrm{s}$ law.

Let's briefly consider the properties of the atmospheric layer, the nearest to the Earth, taking into account the specificity of the discussed problems. The surface layer of the atmosphere - the troposphere - spreads from the Earth's surface to the altitudes of $10-12 \mathrm{~km}$ over the middle latitudes. There are $80 \%$ of total mass of the atmosphere in the troposphere. It should be noted, that the discussed changes of the elements of the atmospheric electricity are mainly conneoted with the change of the troposphere state.

Historically, basic views in atmospheric electricity were characterized by the different answers to the question whether here does or does not exist a close interrelationship between atmospheric electricity and meteorology. It was founded that atmospheric electric field has a periodic diurnal variations and this variations is more clearly marked in "fair weather". This led to distinction between "undisturbed" and "disturbed" periods. In the practice of atmospheric electricity the 
periodic variations of the respective elements have always been closely studied. For the geophysical elements four preferred types of periodically reourring fluctuations are possible: a) diurnal periods; b) 27-day periods; c) annual periods; d) 11-year periods. The above are associated with the movements of the earth relative to the sun ( $a$ and $c$ ), or with the solar periods of the sun revolution (b) and sunspot activity (d). In the atmospherio electricity the "terrestrial periods" $a$ and $c$ are very strongly pronounced, whereas the "cosmic periods" 0 and $d$ only weakly or not at all.

Till recently there was the notion, that troposphere is practically isolated from the higher layers, i.e. from the ionosphere, where the air ionization is determined by ultra-violet rays from the sun and its diurnal change is immediately conditioned by the solar time. But lately, it was founded some experimental lacts [Israel, 1973; Krasnogorskaya and Remizov, 1973; Zybin et al., 1974; Sheftel and Chermyshov, 1992] pointing out the relation between some elements of the atmospheric electricity, such as pulsation of the gradient of the potential of the tropospheric eleotrio lieid, and geomagnetic pulsations as well as mentioned above periodic variations.

Conductivity of the atmosphere is determined by the presence of the mobile charged particles. These particles are ions, which are generated under the influence of the cosmic radiation and radioactive materials, presented in the soil and air. Usually, the ions are classified on the base of their mobility. So, all atmospheric ions might be divided into five basic groups. Radii of the ions for all mobile groups are classified as condensation nuclei and aerosol particles. As a rule, these particles are charged due to the diffusion processes, selective adsorption of the ions, electrification because of the particles destruction and some other causes [Fux, 1955, Frencel, 1949, Leb, 1963]. In the case of the air moistening or appearance of particulate matter (such as dust, smoke, exhaust gases and so "on) in the air, initial concentration of the small ions reduces because of the attachment of such ions to the particulate matter [Tammet, 1975]. 
In the theory of the atmospherio eleotricity the downwards direction is considered as a positive direction of the vector of the eleotric field strength. So, the Earth's surface has a negative electric charge, whereas the positive charge, which compensates this negative electric charge, is in the atmosphere. To describe a large part of processes in the surface layers of the atmosphere it is conveniently to consider that the electric field has only vertical component $E_{z}$, whereas its horizontal components $E_{\mathrm{x}}$ and $E_{\mathrm{y}}$ are negligible.

The mean value of the electric field strength near the ground varies greatly from one point to another. The main reason for the variability of the mean field strength near the ground is that the atmospheric electric lield is a complex quantity simultaneously subjects to universal and local influences. The mean value of the electric field strength near the ground is about of $130 \mathrm{~V} / \mathrm{m}$ and depends on geographical latitude of the point of observations and the orographic location of the station.

Atmospheric electric studies carried out by the Carnegie Institute at sea indioate a clear dependence of potential gradient on geographical latitude. This effect above the sea can be understood as the consequence of the earth's magnetic field [Israel, 1973]. The largest values of $E_{z}$ are observed at the high latitudes and these values decrease in the direction of the poles and the equator [Tverskoy, 1949, Israel, 1973]. A corresponding latitude effect of the potential gradient must also be expected above the land, al though it is probably masked by stronger influences of the local factors.

Moving away from the Earth's surface the value of $E_{\mathrm{z}}$ decreases and at the altitudes of $5-10 \mathrm{~km}$ it is not greater a few $\mathrm{V} / \mathrm{m}$.

Electric field above the given point of the Earth's surface isn't constant. It changes in time depending on the cloudiness, presence of aerosols and other disturbing factors, day time (diurnal variations) and seasons (annual variations). These periodical variations obviously stands out against a background of more significant, but non-regular, changes of $E_{z}$, which are conditioned by change of meteorological conditions and some other disturbing factors. 
Currents in the atmosphere are determined by the transfer of the electric charges, presented in the atmosphere, under the influence of eleotric as well as mechanical forces. In atmospheric electric undisturbed precipitation-free weather we must distinguish between the air-earth conduction current (density $j_{L}$ ), which is mainly carried by small ions, and the air-earth convection current (density $j_{c}$ ). The current density $f_{L}$ is given by

$f_{L}=E_{z} \cdot \lambda$,
where $E_{2}$ is the field strength, $\lambda$ is the conductivity.

The total air-earth current (density $j$ ) is additively composed of two fractions:

$$
j=j_{L}+j_{c}=E \cdot \lambda+j_{c}
$$

During precipitation $j_{c}$ in addition contains the precipitation current.

Conduction current conditioned by the motion of the ions under the action of the electric field forces are on an average about $2 \cdot 10^{-12} \mathrm{~A} / \mathrm{m}^{2}$. Under undisturbed weather oonditions the conduction current seeks to keep the constant value. So, usually, the character of the diurmal variations of the conductivity $\lambda$ is opposite to the change of the eleotric field [Tverskoy P.M., 1949]. The follow equation:

$$
j_{L}=\lambda \cdot E_{z}=\text { const }
$$

allows in this special case to reduce all changes of the atmospheric electricity elements to the change of single value, $E$ for example. But the condition of the current $f_{L}$ constancy isn't exaot due to great number of the factors, whose aotion results in the disturbanoe of the stationary conditions. The presence of space charges in the atmospheric layer, nearest to the Earth, is one of these causes.

During consideration of the electric field in the atmospheric layer, nearest to the Earth, it's necessary to take into account the electrode effect [Chalmers, 1967, Krasnogorskaya, 1972], whioh is conditioned by changes in the mechanism of the current near the conductor surface, because only ions with opposite sign oan mu down on this surface (i.e. only those ions, which are attracted by the surface). For example, if the Earth's surface in cloudless weather has negative charge, the current on this surface might be 
transferred only by positive ions. So, the current density near the surface is described by the multiplication of $E_{2}$ and $\lambda_{+}$, rather than by the convenient expression $\lambda \cdot E_{z}$. In result, the space electric charges forms in the layer, nearest to the Earth.

More full theory of the electrode effects might be received from the expression:

$$
J=\left(\lambda_{+}+\lambda_{-}\right) E_{z}+D \frac{\partial \rho}{\partial z}
$$

The first term in the right part of this expression represents ordinary conduction current, and the second term is the current of the diffusive type, conditioned by the turbulent diffusion in the atmospheric layer, nearest to the Earth. Quantitative estimations [Morozov, 1984, Willet, 1978] illustrate the influence of the turbulent diffusion coefficient and the thickness of the atmospheric layer, nearest to the Earth, on the current density $g$ and electric field strength $E_{z}$. These estimations show, that the value of $E_{z}$ near the Earth's surface changes generally because of the change of the electric charge density $\rho(z)$.

1.2. Measurement techniques of electric field in the surface layer of the atmosphere.

Numerous devices have been developed to measure the atmospherio electric field or potential gradient. With few exoeptions these can be divided in two groups, distinguished by the fact that the first measures potential differences and the second the field strength [Tverskoi, 1949, Imyanitov, 1958, Chalmers, 1967]

Early in the development of measuring techniques, researchers used a potential probes to measure the atmospheric electric potential gradient. This method works with the aid of the so-called "potential equalizer" (potentialsondes, collectors), which is required to fix the "neutral line" of the instrument casing at a certain point. If the conducting body is brought into the field in insulated fashion, than as a mle a certain surface charge will appear at its "equalization point". This charge is removed by the equalization process of the collector [Israel, 1973]. 
In practice the following collector types have been or are in use: Needle oolleotors or point colleotors; Dropper and atomizer collectors; Flame collectors (fuses), and radioactive collectors.

We outline the radioactive collectors. The method of operation is based on their ionizing the surrounding air. When this happens, the ions one charge sign displace themselves away from the collector due to effect of the electric field, while the ions of opposite charge sign move toward it. The movement of charge occurs until the neutral line passes through the equalization point [Israel, 1973].

In the case of radioactive collector the generation of ions in the surrounding air is effected by radioactive rays. An alpha-particle radiating substance is electrolytically deposited on a small metal plate and may be covered with a thin coating of lacquer for protection against weather influences, and particular against hydrometeors. Most widely employed are pure alpha sources (e.g., polonium with a half-life of 138 days or americium 241, nearly 8000 days) or substances, in which the alpha radiation dominates the other radiation components.

Experience with various collector types has shown that only the water dropper collector strictly behaves in accordance with the theoretical considerations and deviations. All potential probes operation on the principle of artificial ionization possess properties produce deviations from the expected behavior and render them dependent on certain precautionary measures. The errors or disturbances are in all cases a result of influences of the air movement on the ionization generated by the collector.

The application of the potential probes with absent radioactive radiation near the ground requires very large values for resistance between measuring element and the ground. Crozier [1963, 1965] has developed a method to measure the atmospheric electric potential: with electronic feedback and the application of the guard-ring principle he obtains an extremely high insulation between his antenna and ground. He used a long wire as a passive antenna connected to an electrometer and amplifier.

Yerg and Johnson [1974] have developed an electrometer to 
measure the voltage on a copper screen used as a passive antenna $1 \mathrm{~m}$ above the ground. The field measuring system provided data concerning the time variation of the atmospheric potential gradient in the range of frequencies between 0.004 and $0.06 \mathrm{~Hz}$. The electrometer is considered to be an ultra-high-impedance voltmeter with a unity gain appropriate for measurement of voltages where source impedance exceeds $10^{9}$ 8. The electrometer has a range of about $0-300 \mathrm{~V}$; the input resistance is about 100 times the resistance of air $\left(2 \cdot 10^{15} \Omega\right)$, and the input capacitance is less than $10^{-14} \mathrm{~F}$.

The measurement techniques of electric field and currents in the atmosphere have been analyzed by Ogawa [1973]. Practical uses of these techniques as well as various sources of atmospheric electric fields and their characteristics are briefly described.

Here we consider in detail the antenna method to measure the atmospheric electric field.

In the antenna method we measure a potential difference between two conductors in the electric field. When it is applied near the ground surface, one side of the conductors may be the ground. For the convenience of analysis, let a pair of parallel plates be insulated, and placed separately in the direction of electric field in the air of conductivity $\sigma$ and of electric field $E_{0}$. In the following analysis the plates are assumed to be so large that the edge effect is neglected. The potential applied between the plates is

$$
V_{0}=E_{0} \cdot n
$$

where $h$ is called an effective height, and in this case is equals to the true separation $d$, i.e.,

$$
n=d \text {. }
$$

Between the two plates is connected a parallel $R C$ circuit which is an input of the amplifier. The charges induced on the antenna capacitance $C_{\text {。 }}$ and the amplifier input capacitance $C$ after enough time elapsed since the connection, are shown in Figure 1.1.

The relation of the antenna effective height $h$ to the true separation $d$ is given by

$$
n<d \text {. }
$$

In order to minimize the difference between $h$ and $d$, and the 


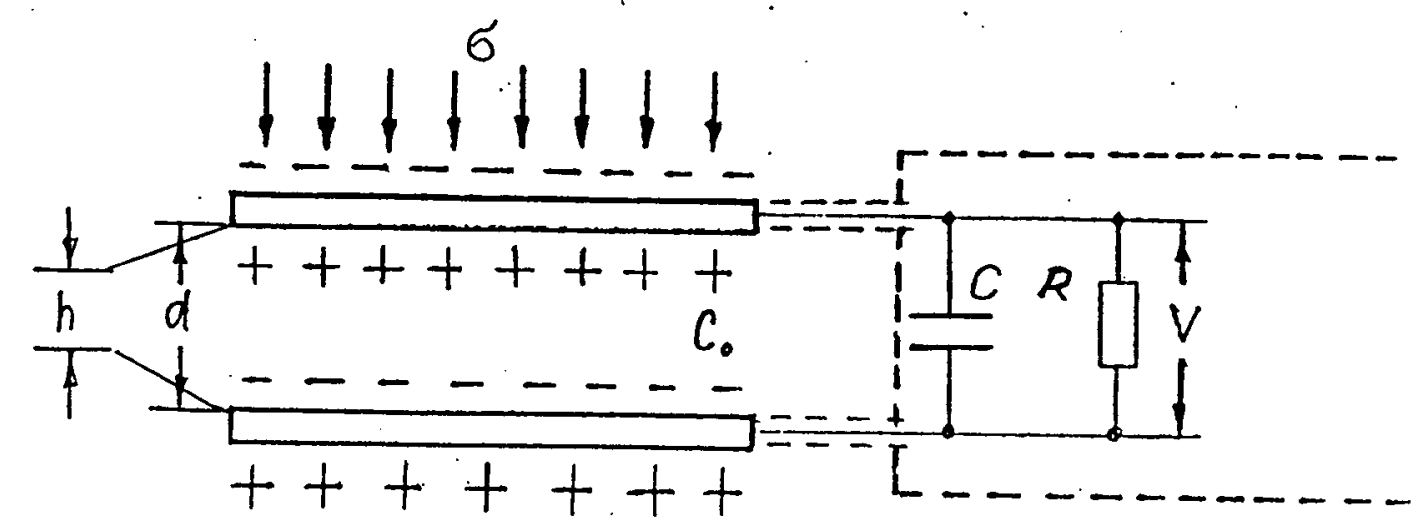

Figure 1.1. Schematio diagram of the parallel plate antenna.

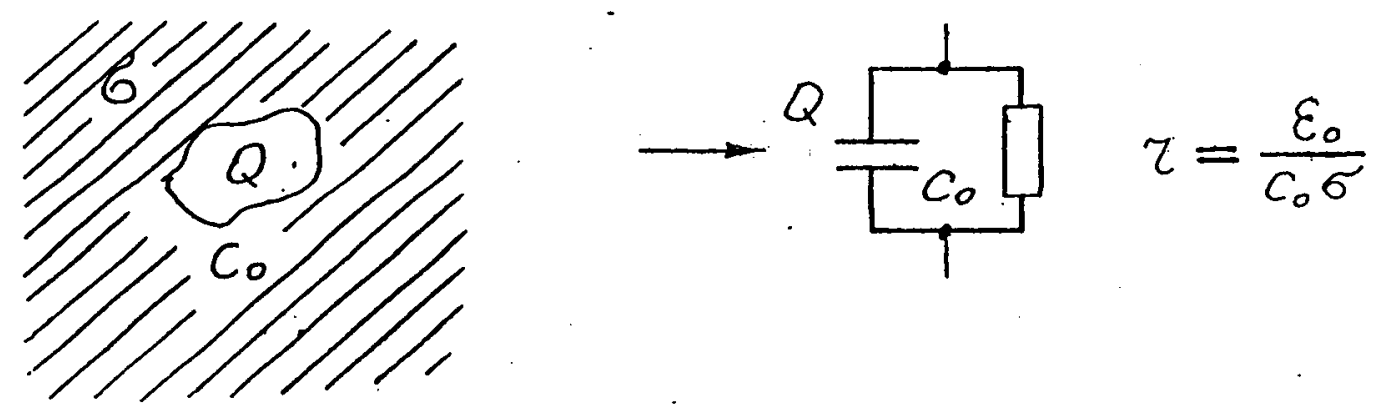

Figure 1.2. Equivalent curcuit of a passive antenna. 
electric field deformation due to adding of $C$, it is intuitively recognized in Figure 1.1 that $C$ is required to be much smaller than the antenna capacitance $C_{0}$. Then

$$
c \text {. } \gg C
$$

This condition will finally be derived from the following analysis. Therefore the antenna effective height is assumed to be equal to the antenna true separation. Let the charge on $C$ be $Q_{t=0^{-}}$, then the charge on $C_{0}, Q_{t=0^{-}}$is given by

$$
Q_{t=0^{-}}^{\cdot}=\frac{C_{0}}{C} Q_{t=0^{-}}
$$

Now let the extermal electric field change suddenly from $E_{0}$ to $E$ by step function. The charge on $C$ at this instance $\left(t=0^{+}\right)$ $Q$ is given by

$$
Q_{t=0^{+}}=Q_{t=0^{-}}+\frac{C}{C+C_{0}^{-} \varepsilon_{0}}\left(E-E_{0}\right) S,
$$

where $S$ is the area of the plates. The charge changes with time depending on (1) the conduction current $\sigma E$ flowing into the plates from the extermal atmosphere, (2) the conduction current between the antenna plates $\sigma q / \varepsilon_{0}$ ( $q$ is the charge density on the inner surfaces of the antenna), and (3) the current through the resistance, $R, V / R$, where $V$ is the amplifier input voltage. In the above consideration an effect of displacement current is neglected. Thus the time change of the charge on $C$ is expressed by the differential equation

$$
\frac{d Q}{d t}=\frac{C}{C+C_{0}}\left(\sigma E S-\frac{\sigma C_{0} Q}{\varepsilon_{0} C}-\frac{Q}{C R}\right)
$$

The current flowing into the plates $I$ is given by

$$
I=\sigma \iint E_{n} d S=\frac{\sigma}{\sigma_{0}} C_{0} V
$$

and the equivalent antenna resistance $V / I$ is given by

$$
r=\frac{V}{I}=\frac{\varepsilon_{0}}{C_{0} \sigma}
$$

Using Eq. (1.7); the capacitance of the parallel plate antenna

$$
C_{0}=\varepsilon_{0} S / d
$$

and the relation $Q=C V, E q . ~(1.5)$ can be transformed to the relation of electric potential. The first term of the right of 
Eq. (1.5) can be rewritten as

$$
\sigma E S=\left(C_{0} \sigma / \varepsilon_{0}\right)\left(\varepsilon_{0} S / d\right)\left(E d / C_{0}\right)=E d / r,
$$

The second term is $-\frac{\sigma C_{0} Q}{\varepsilon_{0} C}=-\frac{V}{r}$.

The third term is $-\frac{Q}{C R}=-\frac{V}{R}$. Then Eq. (1.5) becomes

$$
\frac{d V}{d t}+\frac{(r+R) V}{\left(C_{0}+C\right) r R}-\frac{E d}{(C+C) r}=0
$$

The general solution to Eq. (1.9) is $V=\exp \left(-\int \frac{1}{C_{0}+C} \frac{r+R}{r R} d t\right) \cdot\left\{\int \exp \left(\int \frac{r+R}{C_{0}+C} d t\right) \cdot \frac{E d}{\left(C_{0}+C\right) r} d t+\operatorname{const}\right\}$

The integrations on the right of Eq. (1.10) are carried on, then

$$
V=\frac{R}{r+R} E d+\text { const } \cdot \exp \left(-\frac{r+R}{r R\left(C_{0}+C\right)}-t\right)
$$

From the initial condition Const. in Eq. (1.11) is at $t=0^{+}$

$$
\text { const }=V_{t=0^{+}}-\frac{R}{r+R} E d=\frac{Q_{t=0^{-}}}{C}-\frac{R}{r+R} E d
$$

Substituting Eq. (1.4) into Eq. (1.12)

$$
\text { const }=\frac{Q_{t=0^{-}}}{C}+\frac{\varepsilon_{0} S\left(E-E_{0}\right)}{C_{0}+C}-\frac{R}{r+R} E d
$$

The first term on the right of Eq. (1.13) is obtained from Eq. (1.11) when $t \Rightarrow \infty$, and then Eq. (1.13) becomes

$$
\text { const }=\frac{R}{r+R} E_{0} d+\frac{C_{0} d\left(E-E_{0}\right)}{C_{0}+C}-\frac{R}{r+R} E d
$$

Equation (1.14) is rewritten

$$
\text { const }=\left(\frac{C \cdot C_{0}+C}{C_{0}}-\frac{R}{r+R}\right) d\left(E-E_{0}\right)
$$

Substituting Eq. (1.15) into Eq. (1.11),

$$
\begin{aligned}
& \qquad V=\frac{R}{r+R} E d+\left(\frac{C \cdot 0}{C_{0}+C}-\frac{R}{r+R}\right) d\left(E-E_{0}\right) \exp \left(-\frac{t}{\tau}\right) \\
& \text { where } \tau=\frac{r R\left(C_{0}+C\right)}{r+R}
\end{aligned}
$$


The first term on the right of Eq. (1.16) gives the sensitivity, and the second term is a transient term giving the time response of this antenna system. The time constant of the antenna system is given by Eq. (1.17).

For a practical use of this antenna method the second term on the right of Eq. (1.16) is designed to be neglected, so that

$$
V=\frac{R}{r+R} E(t) d
$$

Equation (1.18) indicates that the time varying electric field can be measured without any time delay or time advance. In this case

$$
\frac{C \cdot c_{0}}{c_{0}+C}-\frac{R}{r+R}=0
$$

From Eq. (1.19)

$$
C_{0} r=C R
$$

This is called by Kasemir and Ruhnke (1958) the condition of phase matching. If $r \ll R$ in Eq. (1.18) then

$$
E=\frac{V}{d}
$$

The electric field is obtained simply and directly from the measurement of $V$. Equation (1.19) can be combined with the Inequality: $r \ll R$ to yield the condition (1.2)

$$
C \text {. } \gg \mathrm{C} \text {. }
$$

This was obtained at the beginning of this analysis. From the conditions (1.2) and $r \ll R$ it becomes apparent that the antenna of relatively large capacitance with the small capacitance and large resistance at the amplifier input must be used for the measurement of the eleotric field. Making the antenna capacitance larger is an opposite condition for making the antenna separation $d$ larger. If we let the second term on the right of Eq. (1.16) be close to zero, then there is no need to consider the antenna time constant.

Although Eq. (1.16) is derived from the analysis of a pair of parallel plates, the antenna capacitance $C_{0}$ is the only coefficient relating to the antenna form. Therefore the result of this analysis can be applied to any form of antenna. Even if the antenna form is complex and the antenna capacitance cannot be estimated, the antenna effective height can be close to the antenna true separation when only conditions (1.2) and $r$ « $R$ are satisfied, and thus the electric field can be measured 
from Eq. (1.21).

The equivalent c1rcuit of the antenna. Let a charged spherical conductor be placed in the atmosphere of conductivity $\sigma$, then the initial charge $Q_{0}$ is dissipated by conduction current in the direction of electric field on the surface of the conductor. The charge on the conductor at any time is given by

$$
Q=Q_{0} \exp \left(-t \frac{\sigma}{\varepsilon_{0}}\right)
$$

On the other hand when the initial charge $Q_{0}$ on $a$ capacitance $C o$ is discharged through a resistance $r$, the charge on the capacitance $C_{\text {。 }}$ at any time is given by

$$
Q=Q_{0} \exp \left(-\frac{t}{C_{0} r}\right) \text {. }
$$

Equations (1.22) and (1.23) have the same form. From this comparison a charged spherical conductor may be equivalent to the circuit in Figure 1.2. Let Eq. (1.22) be equal to Eq. $(1.23)$

$$
\frac{\varepsilon_{0}}{\sigma}=C_{0} r \text {. }
$$

The left hand term is called atmospheric relaxation time, and the right hand term is the time constant of the equivalent circuit. In Eq. (1.23) there is no need to be spherical for the conductor, any form of conductor in the atmosphere can be transferred to the equivalent circuit in Figure 1.2.

It can be proved that $r$ on the right of Eq. (1.24) is obtained by integrating the resistance over the conducting sphere from its radius a to infinity. Let a distance from the center of the sphere be $\rho$, then

$$
r=\int_{a}^{\infty} \frac{d \rho}{4 \pi \rho^{2} \sigma}=\frac{1}{4 \pi a \sigma} .
$$

Considering that the capacitance of the spherical conductor is given by

$$
C_{0}=4 \pi \varepsilon_{0} a,
$$

Eq. (1.24) is derived from Eq. (1.25).

A passive antenna that was constructed on the base of above analysis, were made by OKB of Schmidt Institute of the Physics of the Earth. This antenna have a frequency response of $10^{-3}$ to $20 \cdot 10^{4} \mathrm{~Hz}$. 
Figure 1.3 shows a view of passive antenna with built-in electronics including the amplifier and filter circuits.

A calibration of passive antenna as well as "field mill" will be described below. A typical equivalent out put of the antenna was $\approx 0.026 \mathrm{~V}$ per $\mathrm{V} / \mathrm{m}$ of the applied electric field.

The second method of the electric field strength $E$ measurements is based on the use of the electrostatic induction phenomenon (electrostatic induction method). This phenomenon consists in the formation of the electric charges under the influence of the electric field in the conductor. The surface charge density $\sigma$ and the field strength $E$ at the surface of conductor are related by the expression (in the two system of units )

$$
E=4 \pi \sigma=\frac{\sigma}{\varepsilon_{0}} .
$$

Arrangements in which this principle is used for measurement $E$ form the basis for the various field-measuring machines ("field mill" or "electrostatic fluxmeter").

In the "field mill", there is a fixed test plate that is altemately exposed to and then shielded from the lines of force of the earth's field by a moving grounded cover. The plate is connected to the earth through a resistance $R$ and capacitance $C$ in parallel and the alternating current $i$ to earth is amplified, usually with a circuit tuned to the frequency of the shielding, reotified and measured. The average value of the alternating current $i$ during the exposition or the shielding time period $T$ is determined as:

$$
i=\frac{Q_{0}}{T}=\frac{\sigma S}{T}=\frac{E \varepsilon_{0} S}{T},
$$

where $Q_{0}$ is the surface charge. It's evident, that the current value $i$ is defined by the field strength and depends on the frequency of the test plate shielding from the lines of force of the earth's field.

There are two extreme regimes of the measurements: the resistance regime, when the whole charge from the test plate has time to stream down to the Earth within time $T$, and the capacitance regime, when the charge hasn't time to stream down to the Earth and streams down to the capacitance $C$ with the shielding period and returns to the plate within the exposition 


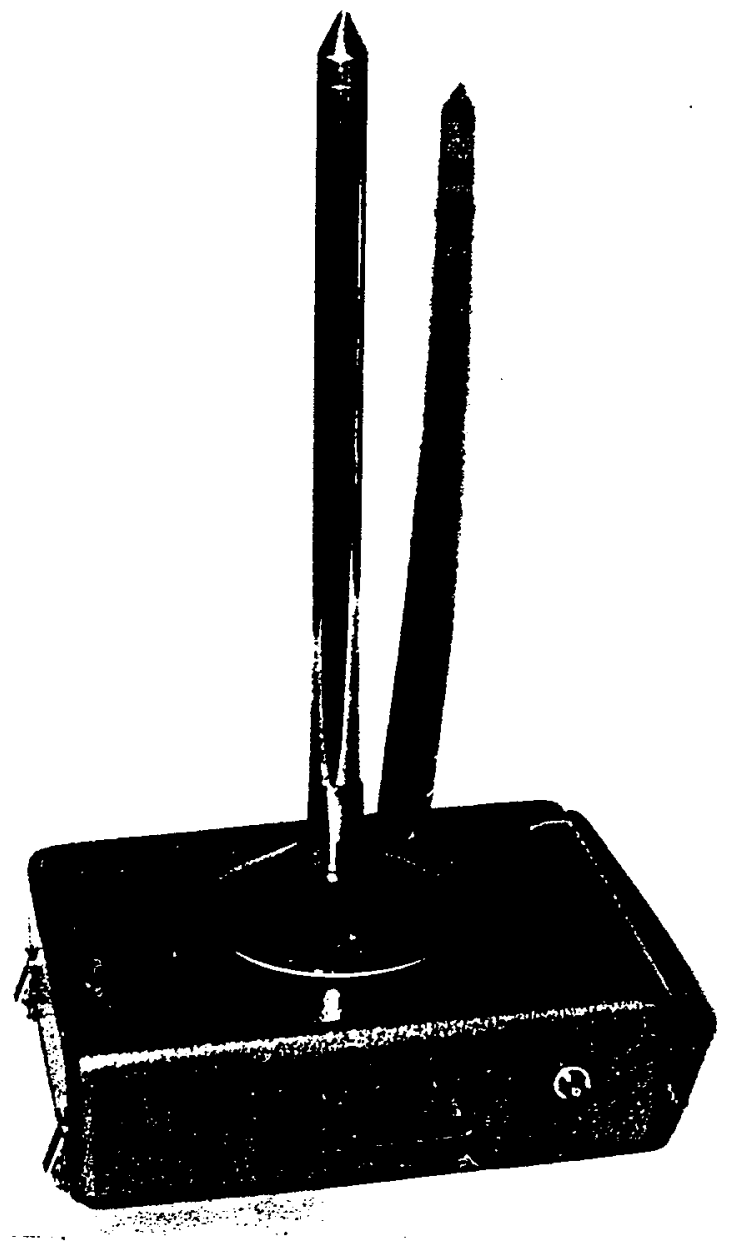

Figure 1.3. A view of passive antenna. 
time. The first regime is realized if $R \cdot C \ll T$, and the second comes true when $R \cdot C \gg T$.

A sensor of the electric field strength that was constructed on the base of above principle, were made by OKB of Schmidt Institute of the Phisics of the Earth. The sensor have a flat rotating cover connected to the ground and two fixed test plates. To increase. the modulation erequency the plates are made as multisectorial. The availability of two, not one, test plates allows to use the symmetrical structure of the entrance amplifier and reduce the synphase noise.

It is convenient to carry out the analysis of the working principle of the this device on the base of the scheme, represented at Figure 1.4. The flat test plate 1 with area $S_{0}$ is connected to the Earth by the resistance $R$. These plate might be exposed in the field $E$, normal to the plate (Fig.1.4a.) or be shielded from it by a moving grounded cover. If the cover rotation is continuous, the current $i$ will run thought $R$ all the time. Let's designate a part of the measuring plate area, which is open for the field influence at the time moment $t$, as $S$, and the oapacitance of the measuring plate with respect to the Earth as $C$. The current through the resistance $R$ creates the voltage decrease $R \cdot t=R \cdot \frac{d Q}{d t}$, where $Q$ is the plate charge. From the other side, the current thought $R$ is created at the expense of the potential difference between the measuring plate and the Farth.

The test plate potential at the moment $t$ is composed from two parts: from the potential $\nabla_{1}=E \cdot S / 4 \pi C$ of the plate in the electric field $E$, and the potential $V_{2}$, which is created by the charge $Q$, formed by the current, running thought the resistance $R$ to the plate. This potential is equal to $V_{2}=-\frac{Q}{C}$. The potential, created by the charge $Q$, has an opposite sign with respect to the potential, created by the field. Then, it's possible to write that $V_{1}-V_{2}=i \cdot R$ or

$$
\frac{E S}{4 \pi C}-\frac{Q}{C}=R \frac{d Q}{d t} \text {. }
$$

Equation (1.27) describes the work of the electrostatic generator, such as "field mill". Its solution shows, that the method, used in this generator, allows to measure the alternating as well as direct fields [Imyanitov, 1957]. 

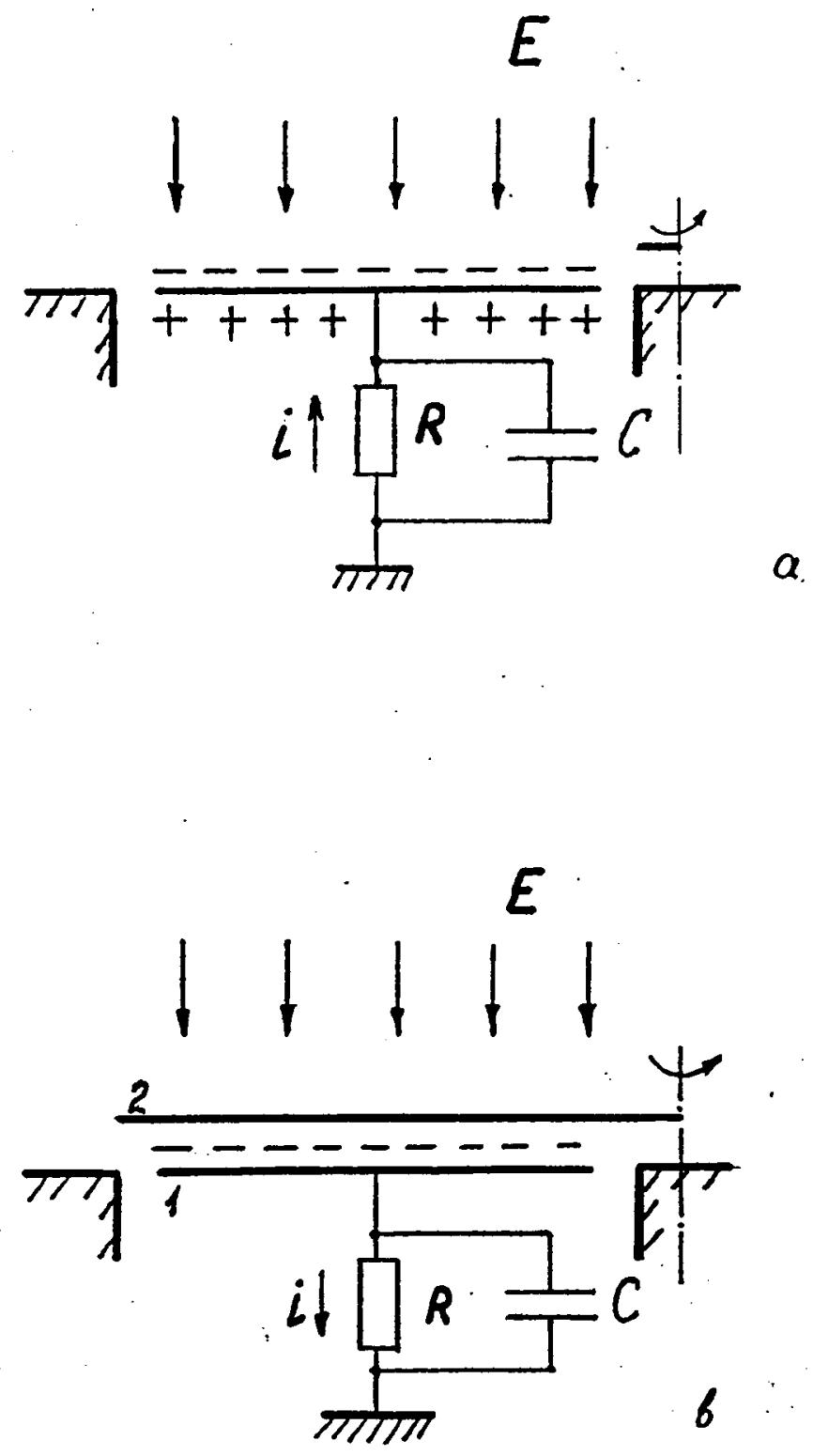

Figure 1.4. Schematic diagram of the "field mill". 1 - test plate; 2- moving grounded cover. 
As a mule the "field mills" are used to measure the electric fields in frequency band from 0 to $10 \mathrm{~Hz}$ [Imyanitov, 1957 Afinogenov, 1977]. During the investigations of the electric field disturbances, generated by the explosion, it's interesting to carry out the registration of the electric field strength in more wider frequency band. The "field mill" as adopted especially for our experiments had a frequency response of $\mathrm{O}$ to $200 \mathrm{~Hz}$. This was achieved by using of multisectorial test plate and moving grounded cover.

The "field mill" was calibrated by putting it beneath a set of aluminum plates. In this configuration, the sensor's plate is placed between the plates of a large capacitor. A known voltage (voltage may be a constant here) is applied across the plates of the capacitor. The field strength at the sensor's plate is easily determined by measuring the output from the "field mill".

Figure 1.5 shows a view of "field mill" with built-in electronics, electrical control unit with amplifiers and filter circuits, and tape recorder.

For the measurement in the atmosphere during the period of fair-weather, the typical equivalent output of "field mill" was $5 \cdot 10^{-3} \mathrm{~V}$ per $\mathrm{V} \cdot \mathrm{m}^{-1}$ of the applied electric field strength.

The eleotrical control unit contained lowpass filtering to pass 0 to $20 \mathrm{~Hz}$, bandpass filtering to pass 0.1 to $20 \mathrm{~Hz}$, and to pass 1 to $20 \mathrm{~Hz}$. These filter circuits helped reduce the noise from the 50-Hz power-line. Total system gain ranged from 1 to 100. In order to measure electric field strength up to 15 $\mathrm{kV} / \mathrm{m}$ we used the built-in amplifier of the "field mill" with adjusted gain.

For the measurement of the electric field in the wide irequency band, we need to make records in relatively narrow band with different gains because of power spectrum decreases with frequency (see below) as $f^{-n}$.

The magnetic sensors that we used during our experiments were made by OKB of Schmidt Institute of the Phisics of the Earth. This sensors consisted of a coil with $\mu$-metal core. The core is composed from a few sections with $400 \mathrm{~mm}$ in length and $15 \mathrm{~mm}$ in diameter. The coil includes four sections with 30000 tums in each of them. So, total number of the tums is 120000 . 


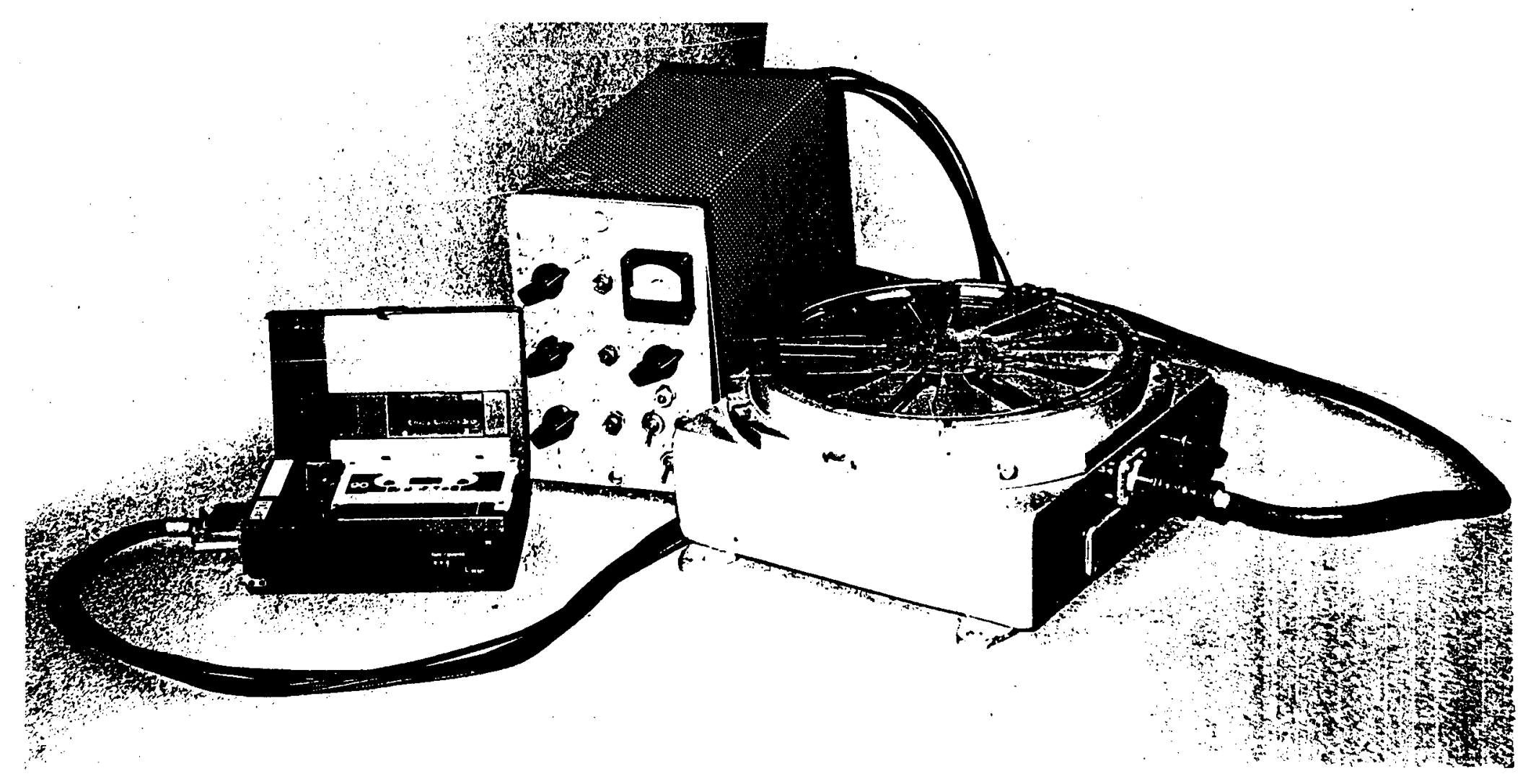

Figure 1.5. A view of "field mill", control unit and tape recorder. 
The sensor has a built-in amplifier. The gain of this amplifier is about 200. The coil and its amplifier were sealed in $a$ water-proof case.

The range of the measured frequencies was chosen from 0.1 to $20 \mathrm{~Hz}$. The upper limit was determined by the necessity to be away from the industrial frequency of $50 \mathrm{~Hz}$.

The typical equivalent output of the inductive magnetometers was $2 \mathrm{~V}$ per $\gamma$ of the applied magnetic field.

We used compact 7 -channel data recorder (Figure.1.5) to acquire data during our observations. TEAC's compact. HR-30 cassette tape recorders are record-only recorders. The recorders are lightweight, portable when battery operated, and have precise drive mechanism. They can record the time and have remote control. A cassette tape recorded by the $H R-30$ can be reproduced by TEAC's MR-30 data recorders.

1.3. Observations of the time variations of the electric field in the surface layer of the atmosphere.

Iike every geophysical quantity the atmospheric electric field is marked by continuous variability. It follows from discussion above that the fair weather electric field above sea, in the Arctic regions and at the continental points remote from the pollution sources usually is positive and has a periodic and nonperiodic variations. In this case a diurnal variations of $E$ have a character of simple wave (single-oscillation type), i.e. there are one maximum, observed about 6-7 p.m., and one minimum about 3-8 a.m. GMT.

We registered the diurnal variations of the electric field strength in some parts of our country with different geographical latitudes. Figure 1.6. represents the diumal variations in Yalta (curve 1, august, 1980, in latitude $45^{\circ}$ North), at Novaya Zemlya (curve 2, September, 1982, in latitude $73^{\circ}$ North) and above oceans (Tverskoy, 1949; Israel, 1973]. The character of the curves 1 and 2 is apparently explained by local effects, which depend on the local time. The diumal variation above the sea is govermed by universal time and it occur simultaneously over the entire earth.

From the observations of the atmospheric electric field 


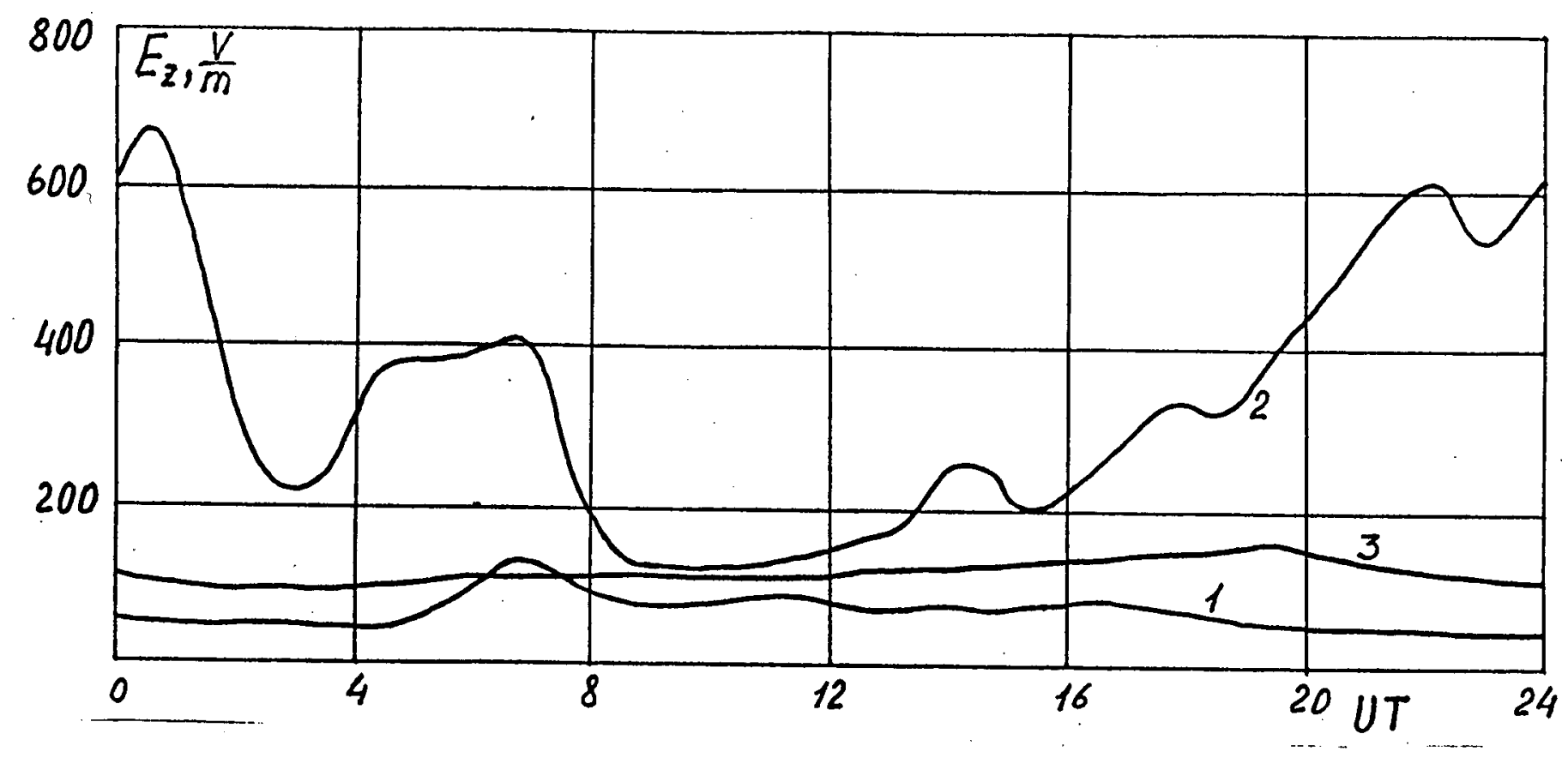

Figure 1.6. Diumal variations of the electric field in the surface layer of the atmosphere. 1- Yalta;

2- Novaya Zemlya; 3- above oceans [Tverskoy, 1949, Israel, 1973]. 
[Chalmers, 1967, Krasnogorskaya, 1972] it's known, that the amplitude and the frequency of the electric field variations depend on the weather conditions, which determines the disturbances of the electric field at the point of the observations. Cloudiness, precipitation, space charge in the air, atmosphere pollution by aerosol and so on might be the sources of the local disturbances of the atmospheric electric field. So, during the measurement of $E_{z}$ it's necessary to carry out simultaneously the observations of the cloudiness, the wind velocity, the presence of a mist or precipitations. Each of these factors is the cause of typical disturbances. Cumulus cloudiness results in the variations with a characteristic period of a few minutes. When the wind velocity increases up to $5-10 \mathrm{~m} / \mathrm{s}$ it's observed the rise of the intensity of the spectral components in the frequency band from 0.1 to $5 \mathrm{~Hz}$.

It's very difficult to carry out the observations in clouds or precipitation [Chalmers, 1967, Krasnogorskaya, 1972]. The influence of mists on the electric field strength consists in the change of the conductivity in the mist in comparison with the conductivity of the clear air. In the atmosphere the attachment of the small ions to the mist particles reduces their mobility enormously and makes them essentially ineffective in transporting charge. Evidence of the importance of particulate matter in reducing atmospheric conductivity is found in the long-term records at Kew (Iondon) [Chalmers, 1967, Israel, 1973]. The average conductivity in the mist is lower by a factor of 3-10 than its value in very clear air. It results in increase of $E$ in the mist in comparison with the value of $E_{z}$ in the clear air. So, it's desirable to study the electric field and its variations under the fair weather conditions, which include the next criteria: the absence of thunder-storms, a strong wind, any types of precipitation, a hour-frost, a mist, a drizzle, a cloudiness, especially cumulus cloudiness.

Even in fair weather the short-periodical variations of the electric field are put on the unitary variation [Chalmers, 1967]. Simultaneously with the registration of the diurnal variations of the electric field strength we registered an 
aperiodic variations (fluctuations) of $E_{z}$ at the stated points. On the base of these records we studied the spectral characteristics of the fluctuations of the electric field $E_{2}$ in the frequency range from $0.6 \cdot 10^{-3}$ to $0.4 \mathrm{~Hz}$ [Krasnogorskaya and Soloviev, 1984].

In order to estimate the correlation function and power spectrum the outputs of the electric field sensors were recorded for 1-8 hours. Analog to digital conversion with interval $\Delta t$ of $2.4 \mathrm{~s}$ and $1.25 \mathrm{~s}$ was carried out during the treatment of these records. Smooth spectral estimation of $S(f)$ were received by means of using of the Tukey correlation window with the width of the frequency band of $b=1.33 / m \cdot \Delta t$, where $m$ is the number of the correlation function points [Jenkins and Watts, 1969]. In spite of the fact, that the estimation of the spectral density were received with interval $\Delta f=(2 \cdot m \cdot \Delta t)^{-1}$, the frequency resolution is determined by the distance between two non-correlated estimations of $S(f)$ due to the smoothing. The resolution is equal to the width of the frequency band of the spectral window. So, all features of the frequency spectra of the electric fluctuations might be considered as statistically substantiated when their width is greater or equal to the width of the spectral window.

Initial records were preliminary subjected to the low-frequency filtration in order to remove low-frequency trends and spectrum distortions, connected with these trends. Smooth spectral estimations are random values. Their probability density has $\chi^{2}$-distribution with $v$ degrees of freedom and they demand to determine the confidential intervals. For Tukey window, the number of $v$ of the smooth spectral estimation is equal to $\nu=2.667 \mathrm{~N} / \mathrm{m}$. The confidential intervals with the demanded probability and known $v$ might be found from $\chi^{2}$-distribution tables (the confidential intervals with 95\%-probability are shown at Figure 1.7).

During the treatment the record length was equal to 1500 points, and the length of the correlation function was given in the interval from 100 to 200 points. The width of the spectral window was changing from 0.0056 to $0.0026 \mathrm{~Hz}$, and the number of the freedom degrees was changing from 20 to 40 , correspondingly. 


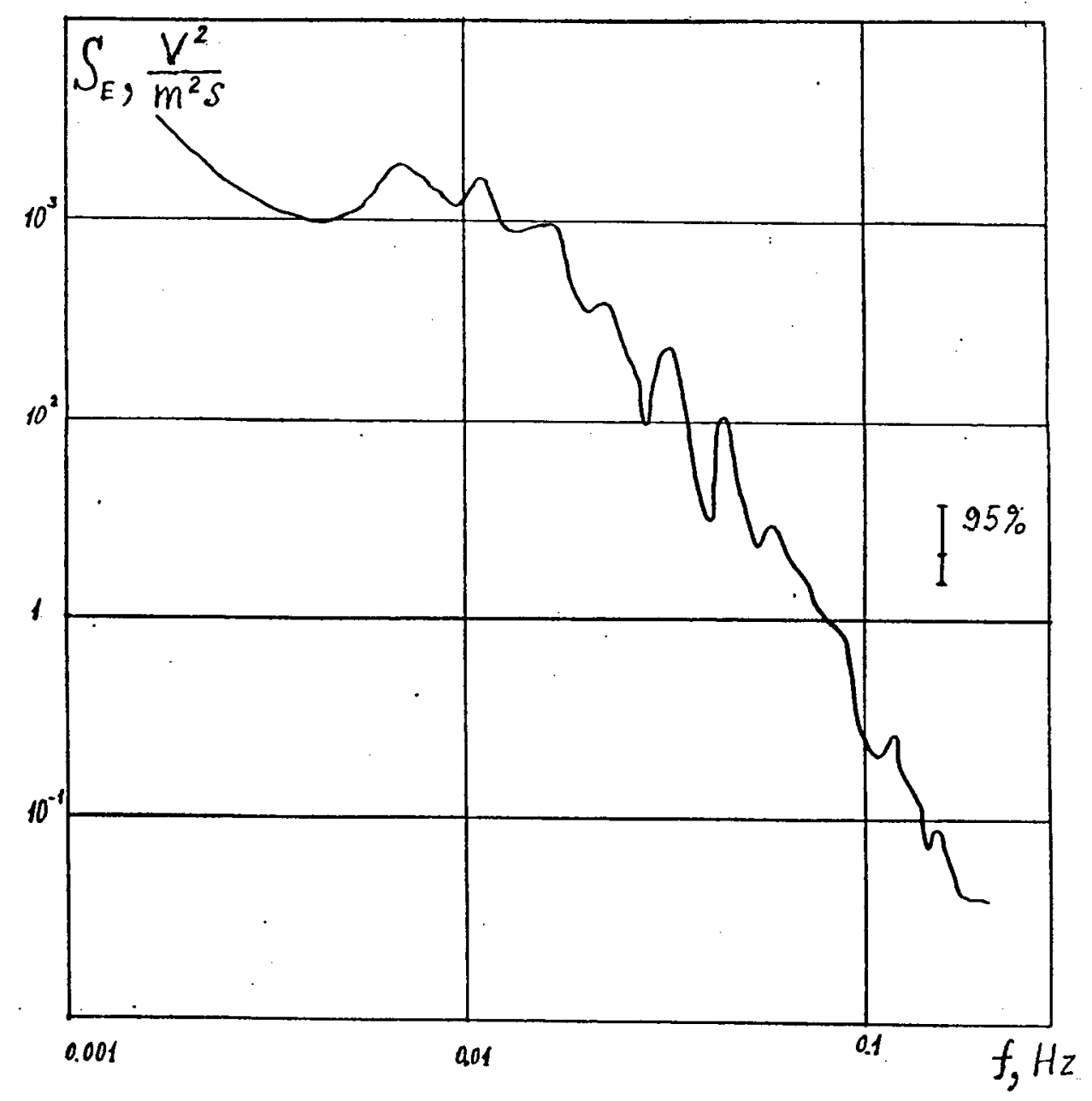

Figure 1.7. Typical power spectrum of the electric field variations in the surface layer of the atmosphere. 


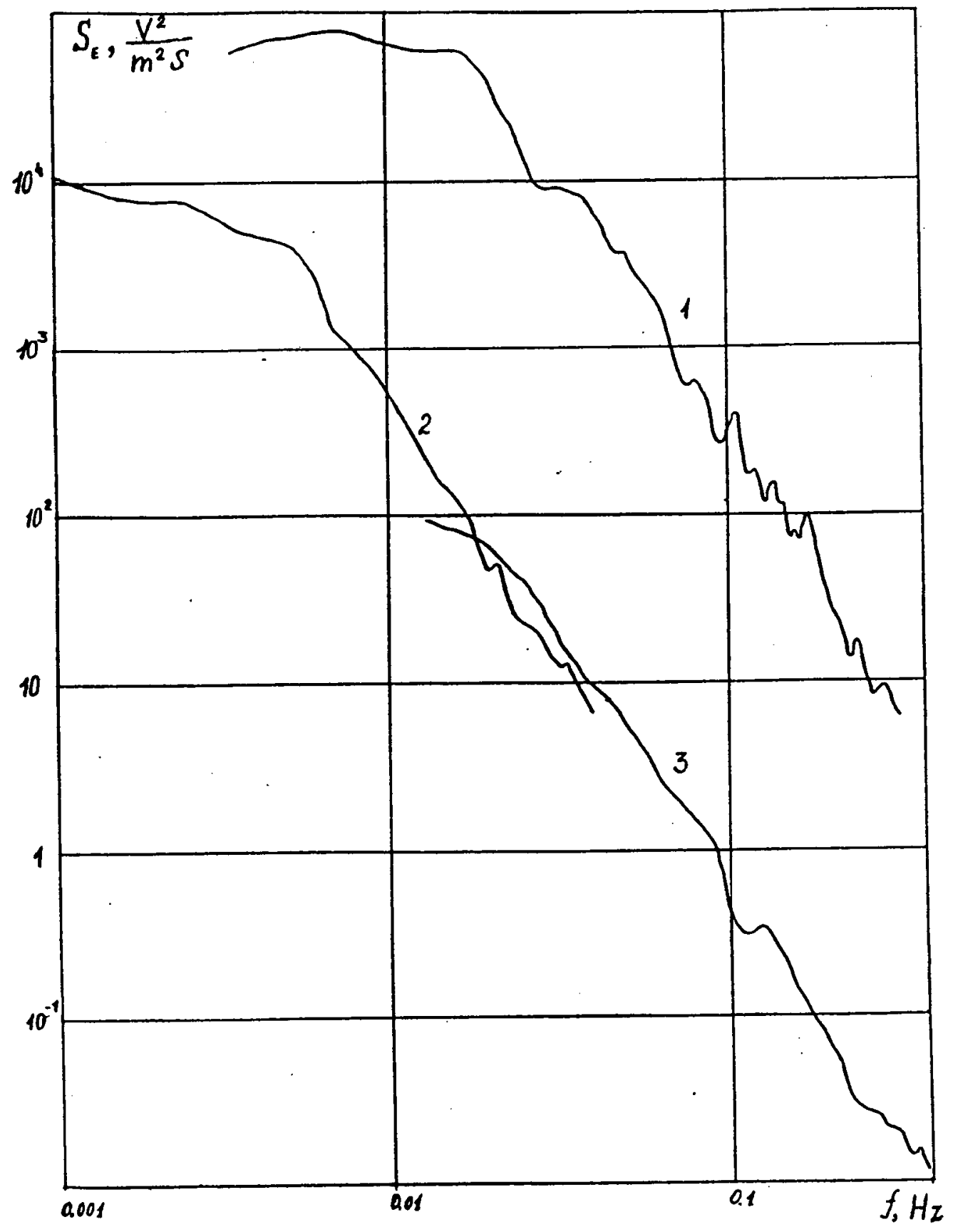

Figure 1.8. Typical power spectra of the electric field variations in the surface layer of the atmosphere. 1,3- one hour records; 2- eight hour records; 1- Novaya Zemlya; 2,3- Yalta. 
The most typical power spectra of the electric field pulsations $E_{z}$ in the boundary surface layer are represented at Figure 1.8. One can see that the power spectra have a distinct peaks which have a sufficient resolution by the frequency. These peaks are the characteristio feature of the spectral density behavior in this frequency band. As is evident from the graphs, the dependence of the spectral density on the Irequency might be approximated by the next power function:

$$
S(f) \simeq f^{-n}
$$

where the value of $n$ is about 3. But in order to determine the value of the power $n$ in equation (1.28) with higher accuracy, it's necessary to receive the estimations of the spectral density $S(f)$ with the frequency less $0.025 \mathrm{~Hz}$. With this aim we carried out the treatment of the records of the changes of $E_{z}(t)$ with duration 8 hours. Figure 1.8 represents the power spectra of the electric field pulsations $E_{z}$, received by the treatment of $E_{z}(t)$ records with duration of 1 and 8 hours (Yalta, august, 1980). As is obvious from Figure 1.8 the dependence of the spectral density on the Irequency in the Irequency band from 0.006 to $0.4 \mathrm{~Hz}$ is approximated by the power funotion of type (1.28) with $n \bumpeq 3.1$. Decrease of the spectrum slope is observed at the frequencies less $0.006 \mathrm{~Hz}$. Except the mentioned dependencies, Figure 1.8 represents the frequency spectra of the electric field pulsations $E_{2}$, which were received from the hour-long records, registered at Novaya Zemlya, 1982. It's evident, that at these spectra the spectrum slope is constant, whereas the energy is considerably greater than this value for the previous case.

1.4. Disturbances of the atmospheric electric field from the sources of man-made and natural origin.

Thunderclouds and electric discharges, connected with them, dust storms, eruptions of volcanos are most strong natural sources of the disturbances of the atmospheric electric field. A lightning is an electric discharge (a very high speed process)

with a length from a few tens of meters to a few kilometers. Electric charges, which are spatially separated in 
thunderclouds, are the most wide-spread sources of a lighting origin. Nore than a half of all discharges take place inside the cloud. If in the cloud the charge with the same sign concentrates over the charges with opposite sign, than this cloud is called "bipolar", and it's assumed, that this cloud has a positive polarity if the positive charge are above the negative charge.

Measurements of the potential gradient near cloud is one of the methods to receive the information about the electricity of the thunderclouds. Under these conditions the values of the potential gradients are significantly greater than under the conditions of fair weather. As it was mentioned above, cumulus clouds are the cause of the typical disturbances of the electric field with a period of a few minutes. The electric field decreases under a cumulus cloud and even may to change its direction at the opposite in comparison with the normal field, corresponding to the clouds absence. Results, which were received after the $f l y$ of air crafts with measuring devices through the cloud, showed that, generally, the distribution of the electric charges in the thunderclouds is the follow: the positive charge is located above the cloud, the negative charge is below the cloud. Besides that, there is concentration of small positive charge in the limited part under the negative charge at the bottom of the cloud [Trerskoy, 1949, Chalmers, 1967]. At present there are different estimations of the value of main electric charges in the thundercloud. Some of them give the charge value of $+40 \mathrm{C}$ at the altitude of $10 \mathrm{~km},-40 \mathrm{C}$ at the altitude of $5 \mathrm{~km}$, and $+10 \mathrm{c}$ at the altitude of $2 \mathrm{~km}$. But if one takes into account the change of the conductivity in the cloud in comparison with the environment and its changing with altitude, than the charges values will be significantly greater: the negative charge has a value of order $300 \mathrm{C}$ [Chalmers, 1967].

Measurements of the electric field strength during a lightning flashes give more detail information, because the lightning corresponds to the transmission of the charge from one part of the cloud to another, or from the cloud to the Earth. It is possible to determine the cloud polarity on the base of the change of $E$, conditioned by the discharge at a 
short distance, because we know the cloud, which is connected with this discharge, and the place, where the discharge took place - inside the cloud or on the Earth.

Figure 1.9 shows the change of the vertical component of the atmospheric electric field strength $E_{z}$ recorded by "field mill" at a distance of about $10 \mathrm{~km}$ from the storm-discharge.

Electric and magnetic field of the electric discharge in the thundercloud might be described, if we'Il represent the source of the field as a dipole with variable electric moment $p(t)$. Than the electric field $E$ at a distance $R$ is determined by the follow expression:

$$
E_{r}=\frac{1}{4 \pi \varepsilon}\left(\frac{p}{\mathrm{R}^{3}}+\frac{1}{\mathrm{cR}^{2}} \cdot \frac{d p}{d t}+\frac{1}{c^{2} R} \cdot \frac{d^{2} p}{d t^{2}}\right),
$$

where $p=2 \cdot q \cdot h, q$ is an electric charge, transferred during the discharge, and $h$ is the length of the electric discharge channel.

In this equation it is assumed that $h$ is very much smaller than $R$. The first term of the equation (1.29) describes electrostatic effect of the storm-discharge, which consists in the transmission of the electric charge $q$ from one area to another. The second term of the equation (1.29) is called "inductive component", which is proportional to the current intensity. This term is negligible for real measurements. The third term of this equation describes the "radiation component", which is proportional to the change in current intensity. It is the most significant term at large distances from the source.

Except the electric discharges, which arise in clouds naturally, some causes of lightning of man-made origin are known. The lightning might be initiated by means of the fast introduction of a long electric conductor into the area of the relatively strong electric field. In this case the conductors influence results in the increase of the field strength up to the value greater than the electric breakdown of the air. The possibility of the artificial initiation of the lightning was demonstrated during the launching of small rockets with grounded wires, attached to the term, from the board of the ship in sea [Uman, 1988]. The lightning, initiated during this 


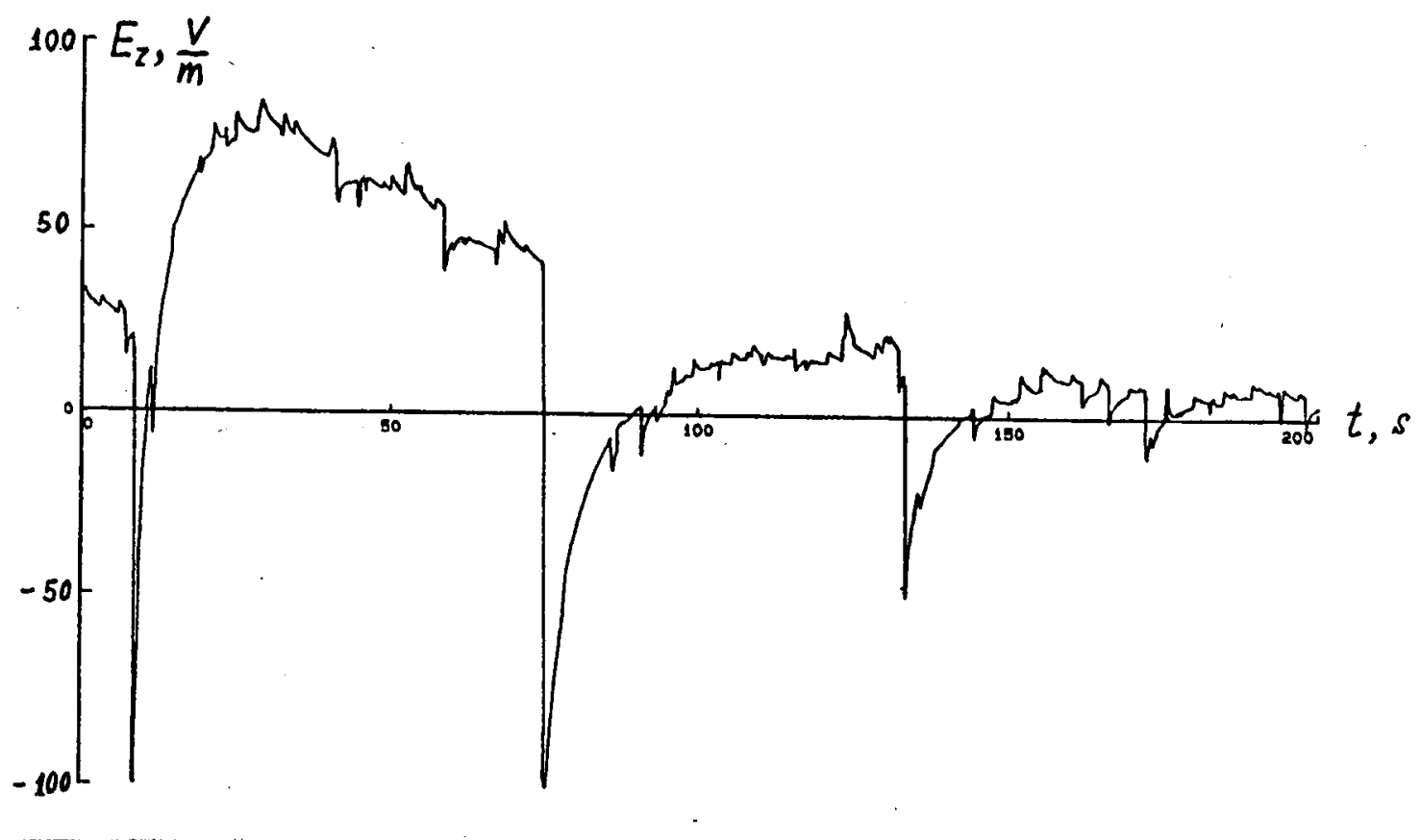

Figure 1.9. The eleotric field $E$ recorded by "field mill" at a distance of about $10^{\mathrm{z}} \mathrm{km}$ from the storm-discharge. 
experiment, were observed even over some land parts. Some cases of the lightning initiation by spaceships and air crafts were registered too [Uman, 1988].

The cases of the lightning initiations by explosions must be noted particularly. After the deep explosion in sea, the output of the water column initiated the lightning with three charges [Brook, 1961, Barri, 1983]. An occurrences of the lightning initiation by the dome's development due to the underground explosion are known too. From these facts we can conclude that the dome has an electric charges on its surface. These charges form an area of the strong electric field, sufficient for the generating of the electric discharge in the air. So, they create the possibility of the lightning initiation under such environment conditions when no natural lightning can arise. The investigations of the configuration of the lightning, initiated by the nuclear explosions near the Earth's surface, showed that this form depends on the spatial distribution of the electric charges [Williams, 1988, 1989]. At the photos of $\mathrm{H}$-bomb explosions, which were carried out in 50-th years, we can see, that often the fire sphere is surrounded by lightning flashes [Williams, 1988, 1989]. In contrast to the thunderclouds, the mechanism of the electric charges separation is well known for these cases. Semi-spherical symmetry of the explosion allowed to create simple theoretical and experimental models of the electric charges distribution and to investigate their influence on the lightning configuration.

Kamra [1972] experimentally studied the electrical properties of dust storms. The experimental data show that all dust storms are electrically active and produce remarkable electrical perturbations in the fair-weather electrical state of the atmosphere. Note, that investigators observed both positive potential gradients and negative potential gradients with magnitudes up to $5-10 \mathrm{kV} / \mathrm{m}$ during nearby dust storms. Visual observation by Kamra [1969] of lightning discharge presumably occurring inside a dust storms indicated the presence there of high electric fields. 
2. Electromagnetic signals generated by detonation of high explosives in the air.

The processes during the detonation of high explosive was studied in connection with astrophysical and geophysical problems, modeling of the high-current radiating discharges, flow of radiating gas round the objects and development of the laser plasma. Electromagnetic effects have been studying not so intensive. To analyze this problem it is necessary to take into account not only gasdynamic processes, but also the symmetry of the real explosion, nonsteady of the flow and, correspondingly, the sharp change of the gas parameters with a distance from the explosion centre. In accordance with stages of the explosion process development we can cut out three main factors, which influence on the electromagnetic signal generation after the detonation time. They are: Detonating head, which is a complex of a detonation front and a chemical reaction zone; Products of detonation, which is formed behind the detonation head; and air shock wave. In a whole, the classification of the processes during the detonation of high explosive, which can generate the electromagnetic fields represented by the scheme show at Figure 2.1 [Selivanov at al., 1990].

Physical model of the process of the electromagnetic radiation from detonation might be described by the system of electromagnetic gasdynamic equations with regard of radiation for the general case of viscous heat-conducting gas [Selivanov at al., 1990 J. But this is very complicated model in order to be used in a practical aims. So, the step-by-step analysis of the process with simplification assumptions is usually used.

On the other hand, the analysis of experimental data which was obtained from the measurements of the electromagnetic radiation from detonation, allows to choose and test some models of the observed processes. Now we'll pay much attention to the description of the experimental studies of the electromagnetic radiation from detonation of high explosive in the air. Conventionally, these studies might be divided into two groups. The first group includes the observations, where the quasistatic fields with frequency range from a few tens 
GENERATION OF ELECTROMAGNETIC FIELD BY DETONATION OF HIGH EXPLOSIVES

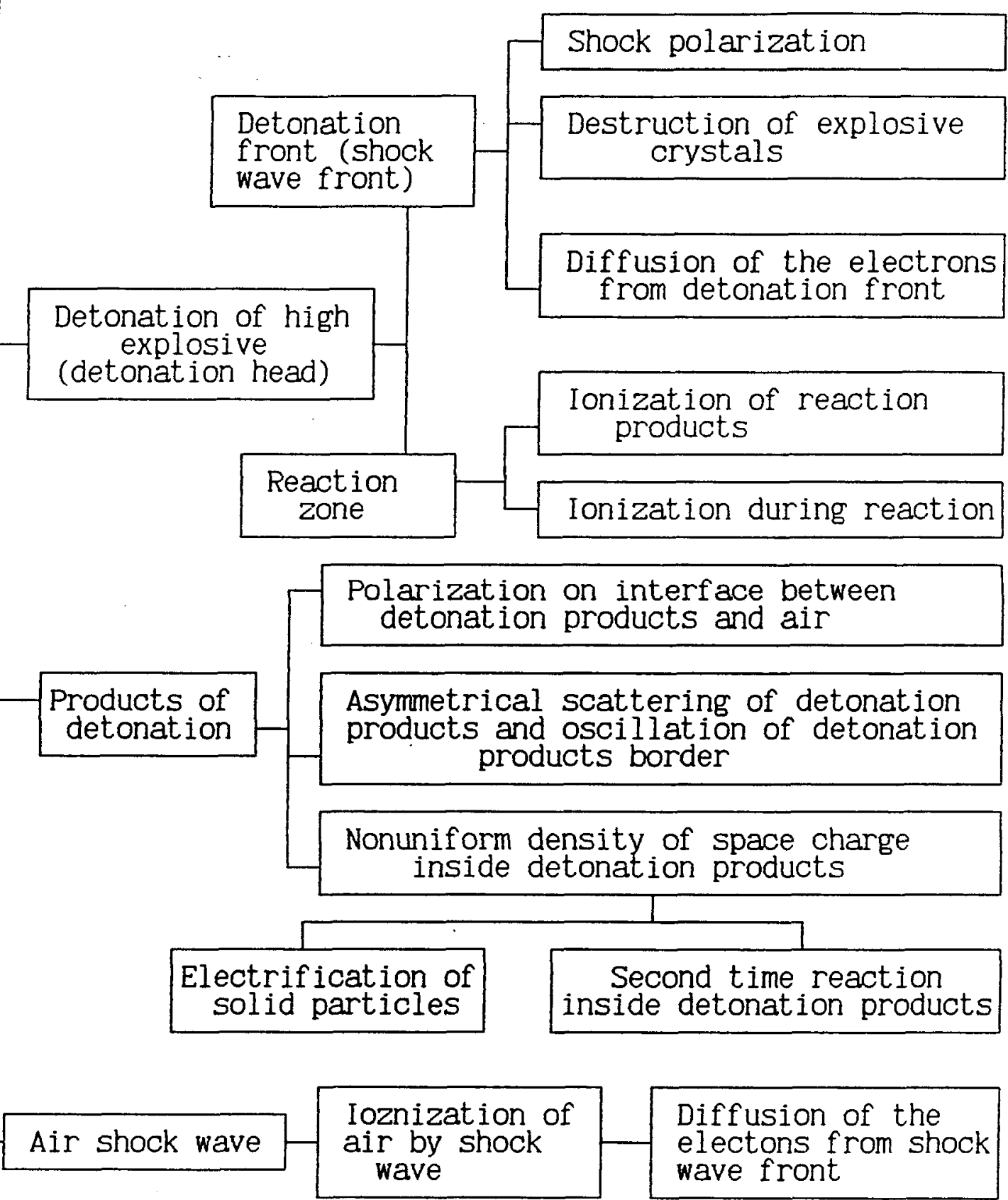

Figure 2.1. Classification of the processes during the detonation of high explosive, which can generate the electromagnetic fields [Selivanov at al., 1990]. 
Hertz to a few tens kilohertz are measured, and the second group includes the observations of the electromagnetic fields with erequencies above $10^{6} \mathrm{~Hz}$.

Let's consider some possible mechanisms of the generation of electromagnetic signals during the detonation of high explosive (Figure 2.1). Electric effects at the zone of the detonation front might be caused by a shock polarization, a diffusion of the electrons from the detonation wave front, a piezo-effect, and a destruction of the explosive crystals. But now there isn't a general conoeption of the apportionment of prior factors which define the generation of the electromagnetic field from detonation.

The phenomenon of the electric polarization of dielectrics behind the shock wave front have been analyzed in detail by Mineev \& Ivanov, [1976]. Since, the condensed explosive, according to its physical properties, is a dielectric (i.e. linear polarized dielectric) [Mineev \& Ivanov, 1976], the shock polarization effect is brought about by the shook compress of the explosive, including its detonation too. Behind the detonation front, there is a gradient zone of sharp compressions and accelerations, where the polarization signals are generated. From the thermodynamic viewpoint, the state of the shock polarization of the matter is nonsteady and it is destroyed with characteristic time of thermal disorientation of molecular dipoles $\tau$.

Change of electric polarization of the matter might be characterized by dipole moment of the shook-compressed zone $P(t)$ changing in time. This change is accompanied by the appearance of the electric and magnetic fields in space around the polarized area. If the dipole moment $P$ changes with characteristic time $\tau$, the length of the radiated electromagnetic wave is $\lambda=c \cdot \tau$, where $c$ is the speed of light. At a distance $R$ from the polarized area, which satisfies the condition $I \ll R \ll \lambda$ ( $I$ is the characteristic size of the polarized area), in the so-called nearest zone (induction zone), i.e. a zone, where wave terms are negligible, the strength of the electric $E$ and magnetic $H$ components of the electromagnetic field of the dipole might be estimated by the following expressions: 

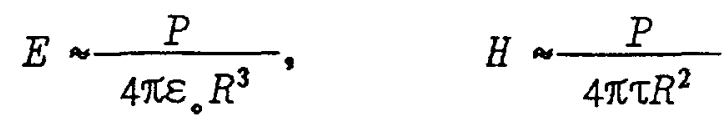

Let's estimate the values of $E$ and $H$ for trinitrotoluene (TNT). Setting the surface density equal p $10^{-5} \mathrm{C}^{2} \mathrm{~m}^{2}, \tau \approx 10^{-8} \mathrm{~s}$, the detonation velocity $D \sim 10^{4} \mathrm{~m} / \mathrm{s}, \quad I=D \cdot \tau \sim 10^{-4} \mathrm{~m}, \quad P=\rho \cdot \tau^{2} \cdot I$ (where $l$ is the characteristic dipole size with value of about model diameter) and $\tau \sim 10^{-2} \mathrm{~m}$. At a distance of $R=100 \mathrm{l}$ we get the values: $E \approx 10^{-3} \mathrm{v} / \mathrm{m}, H \approx 10^{-6} \mathrm{~A} / \mathrm{m}, \lambda \approx 3 \mathrm{~m}$, and characteristic signal frequency range between 10 and $100 \mathrm{MHz}$. These values of $E$ and $H$ are at a few orders smaller than the values recorded in experiments, which correspond to quasi-static field (the characteristic duration of the impulse, registered in the experiments, is about a few milliseconds.

The difference between the results is explained by the fact, that the mentioned components $E$ and $H$ were generated by whole explosion area, which consists of the detonation head, the detonation products, and air shock wave, whereas the estimation taken into account only fields which depended on the polarization zone behind the detonation front. So, the received result gives an idea of the relation between the electromagnetic field generated by the detonation front and total electromagnetic field of the explosion.

In some papers there are an experimental studies of the high-frequency components of the electromagnetic field, generated, apparently, by processes of the charges separation behind the detonation front. Takakura [1955] experimentally studied the short-wave electromagnetic radiation from the detonation of high explosive charges in air. This radiation was measured at frequencies of 3300,190, 90, 16 and $6 \mathrm{MHz}$, and at Irequencies less than $1 \mathrm{MHz}$. The measurements at all frequencies (except the frequency of $3300 \mathrm{MHz}$ ) were made by using two antennae with $l=50-160 \mathrm{~cm}$. At the frequency of $3300 \mathrm{MHz}$ the horm-type antenna was used. The antennae were placed at a distances of 10-200 $\mathrm{cm}$ from the explosion. Takakura used 0.1 to $0.4 \mathrm{~g}$ lead azide charges detonated in air by a flame. The lead azide charges were placed on the wooden base at a height of $30 \mathrm{~cm}$ above ground. Maximum value of the radio-emanation amplitude at a distance of about $40 \mathrm{~cm}$ from the explosion was not greater than $100 \mathrm{muV}$. No radiation was 
observed at frequencies greater than $90 \mathrm{MHz}$.

The author of these investigations [Takakura, 1955] was longing to find some analogy between the mechanisms of the formation of the short-wave radiation by the explosion and the sporadical sun radiation. Evidently, this aim influenced on the general character and orientation of the investigations. The mechanism of electromagnetic radiation from detonations, proposed by the author, is connected with the process of the acceleration or deceleration of electron groups in the ionized layer of air at the shock wave front. This mechanism determined the character of the interpretation of the observed experimental data.

Anderson \& Long [1965] studied the dependence of the electric impulse and short-wave radiation generated by the explosion from the amount of the inert admixtures (gyps, bicarbonate sodium) and the method of their inclusion in the explosive charges. It was noted, that the electric impulse amplitude is directly proportional to the mass of the inert admixtures. The short-wave radiation generated by the explosion was registered in the frequency range of $f=400-500 \mathrm{MHz}$ as a sequence of short-term chaotic splashes. The increase of the explosives mass resulted in the rise of the splashes number, but their amplitudes remained constant. It should be noted, that the conditions of the experiment and methods of the signal registration in this work are still not clearly described.

Kolsky [1954] investigated the spreading of the shock wave in solids. In these experiments the shock waves was generated by the small-scale oharges of high explosives. During the experiments an electromagnetic radiation was measured. To study these radiation the additional experiments were carried out. The aim of these experiments was to investigate the electromagnetic disturbances generated by the detonation of high explosive. To measure the electric component of the disturbarices a passive antenna with length of $10 \mathrm{~cm}$ were used. During the experiments the charges of azide lead and other explosives were placed on metal and dielectric slabs.

The electric pulses which duration were a few milliseconds observed in all experiments. The signal maximum measured approximately at $50 \mu \mathrm{s}$ after the detonation time. The author 
proposed the next explanation of this effect. The detonation of high explosive forms an ionized air layer behind the shock wave front. In this layer the oharges are separated because of the different mobility of the electrons and ions, that results in the formation of some effective electric dipole. Inorease of the radius of the shook wave front and charges recombination lead to the change of the electric dipole moment, and, as a result of this process, the electric field appears in the extermal area of the explosion.

Cook, [1958] carried out studies of electric field radiated from detonations. The method used to detect "radio noise" was simply to measure with a cathode follower the average voltage developed in an antenna. Two antennas were placed at various distances and orientations relative to the axis of cylindrical charges of various sizes. The charges were placed at various heights above ground and fired either with electric blasting caps or fuse cups. The author noted that many results confirm Takakura's observation that the radio noise at a given distance from the charge has essentially the same characteristics independent of the orientation of the antenna relative to the charge axis.

The Irequency of the approximately (single) sine wave pulse from $70 \mathrm{~g}$ to $1.1 \mathrm{~kg}$ charges fired at $90 \mathrm{~cm}$ height above ground with fuse cup varied from 6.2 to $2.8 \mathrm{kHz}$, or about inversely as the one-third power of mass of the charge.

The author noted that the use of a grounded wire soreen $150 \mathrm{~cm}$ above the charge completely eliminated the radio-noise signal. This experiment was made in order to eliminate the influence of the normal vertical potential gradient of the atmosphere. The success of this device in eliminating the radio noise appears to associate the radiating of electrical pulses from detonations in this range with the vertical potential gradient of the atmosphere.

Finally, in every case there was a delay between the instant firing of the charge and the appearance of the initial pulse at the antenna correspondent apparently precisely with the time required for the hot gases to expand to the diameter where they were able to make direct contact with the ground, or with the ground probe. 
Cook assume that the mechanism of generation of the $\mathrm{kHz}$ range signals may be as follows:

During the initial expansion into the atmosphere of the gaseous products of detonation, at the front of which is the plasma the gas cloud becomes charged, as a result of polarization of the plasma at the surface of the expanding gas cloud under influence of the atmosphere's field and/or electrokinetics. After this gas cloud accumulates sufficient charge in this way it may then be discharged by making a direct contact with ground or through a suitable grounded probe. The electrical pulse begins to radiate at the instant the discharge commences, the wave length of the radiated pulse being then roughly proportional to the diameter of the gas discharged and to the conductivity of the ground. When the gas cloud is grounded throughout its expansion by shooting the charge directly on the ground it, however, generates no electrical pulse; nor will it generate an electrical pulse unless it is suddenly discharged after it has had time to accumulate a charge in itself. The effects produced by electric blasting caps may be explained by the lact that the cap lead wires provide a direct coupling to the conducting gas cloud creating a type of oscillator which acts over a period of several milliseconds as a transmitter.

Gorshunor at all, [1967] carried out investigations, which, to a certain extent, supplement the investigations by Cook [1958]. Exoept the general information about the influence of the firing methods and the charge form on the characteristios of the measured signal, this study gave the empirical relation between the moment $t_{\max }$, corresponded to the maximum of the electric pulse, and the mass $m$ of high explosive:

$$
t_{\max }=k \cdot m^{1 / 3}
$$

where $t$ is measured in milliseconds and $m$ is measured in $\mathrm{kg}$, $k=0.7 \pm 0.05$. This experimental material gives an opportunity to suppose that the explosion asymmetry is the main factor in the formation of the electromagnetic disturbances. Gorshunov at all [1967] assumed, that electric charges due to the electrification of the scattering detonation products caused the electromagnetic disturbances. It was noted the absence of 
the influence of the field strength and the polarity of the external electric field on the signal characteristics.

Hertzenshtein and Sirotinin [1970] undertook an attempt of theoretical analysis of the electric field generation on the base of the assumption, that the signal origin is determined by the asymmetric motion of the charged explosion products. From this viewpoint, the mechanism of the electric charges separation is as follows: At the moment when the detonation wave reaches the charge surface, the conductivity of the detonation products decreases sharply. The pressure decrease results in the sticking of free electrons to the ozygen molecules and the formation of the negative ions. After the scattering of the charged detonation products, the distance between the positive and negative ions increases and it is observed the electric charges separation. Also the equivalent scheme, whose parameters simulate the scattering of the charged detonation products and the induction of the charges at the antenna was discussed.

One can see that investigators were used an antenna to measure the electrio field. We dwell on the eleotric field sensor that was described more detail by Selivanov at all, [1990].

In accordance with the physical features of the generation of the electromagnetic impulse from the explosion, the problem of the registration of the electric field must be solved for impulses of order a few milliseconds. It's possible to assume the quasistatic oharacter of the external field, the measurements near the Earth's surface or near another surface with high conductivity is characterized by the predominance of the vertical component of the electric field.

The choice of the passive antenna (pole antenna), usually, is conditioned by the next considerations:

- characteristics of the measured field correspond to the demands of the pole antennas applicability, that is the predominance of the vertical component of the electric field and its quasistatic state;

- simplicity of the construction - a conductive pole with length $l$ and diameter $d$ \&, installed on the Earth's surface or another surface with high conductivity, the 
distance from the surface to the pole base is $b$ * $l$. Boronin et al. [1968], Boronin et al. [1972, 1973], Boronin et al. [1990], give the detail analysis of the electric field generation during the detonation of high explosive in the air. The results of the instrumental observation of the electric field from small-scale explosions are discussed below. These authors discuss the dependence of the electric field with the parameters of the explosion. Successive steps of the explosion's development imaged with a high-speed instrumentation camera offer an opportunity to correlate the motion of the shock wave front and explosion products with electric field generated by the explosion.

Electric field was measured by the antenna in the frequency band from $0.1 \mathrm{~Hz}$ to $10^{6} \mathrm{~Hz}$.

The measurements of electromagnetic fields were carried out during the detonations of the high explosive charges with mass of 54 and $660 \mathrm{~g}$. Antennas were placed at distances of 2.5 $\mathrm{m}$ and $3.5 \mathrm{~m}$. The magnitude of the electric field which was recorded during these experiments (Figure 2.2) ranged from 50 to $300 \mathrm{~V} / \mathrm{m}$. After the treatment of the experimental data, the empirical dependence of the electric field strength from the explosion parameters was found by Boronin et al. [1990]:

$$
E\left(t^{\circ}\right)=1.45 \times 10^{4} \psi\left(t^{\circ}\right) \frac{m}{R^{3}}
$$

where $E$ is the electric field strength, $\mathrm{V} / \mathrm{m} ; m$ is the mass of the explosive charge, $\mathrm{kg} ; R$ is the distance from the explosive charge to the point of measurements, $m ; t^{\circ}=\frac{t}{m^{1 / 3}}$ is the reduced time, $s / \mathrm{kg}^{1 / 3} ; \psi\left(t^{\circ}\right)$ is a dimensionless function of the reduced time, which is the same for explosions with different $m$. The function $\Psi\left(t^{\circ}\right)$ is approximated at the interval $0 \leq t^{0} \leq 6 \times 10^{-3} \mathrm{~s} / \mathrm{kg}^{1 / 3}$ as:

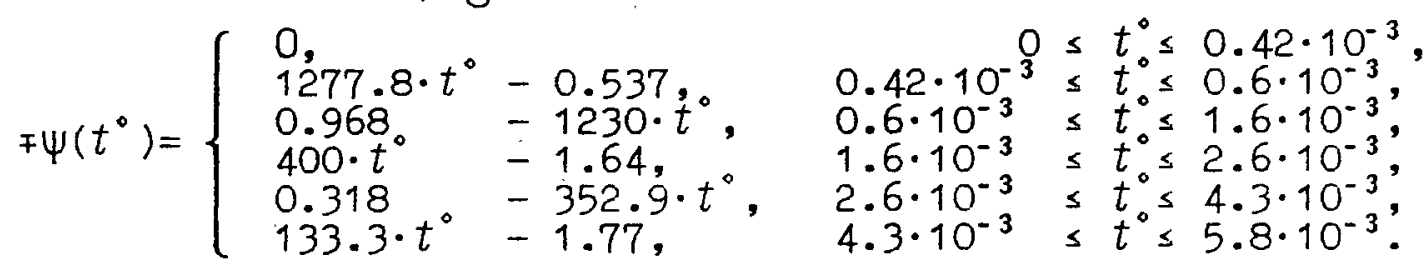

From the comparison of the change in time of the electric field impulse and the radius of the border surface of the detonation products, it was founded, that the electric field impulse oscillation practically repeat the oscillations of the 


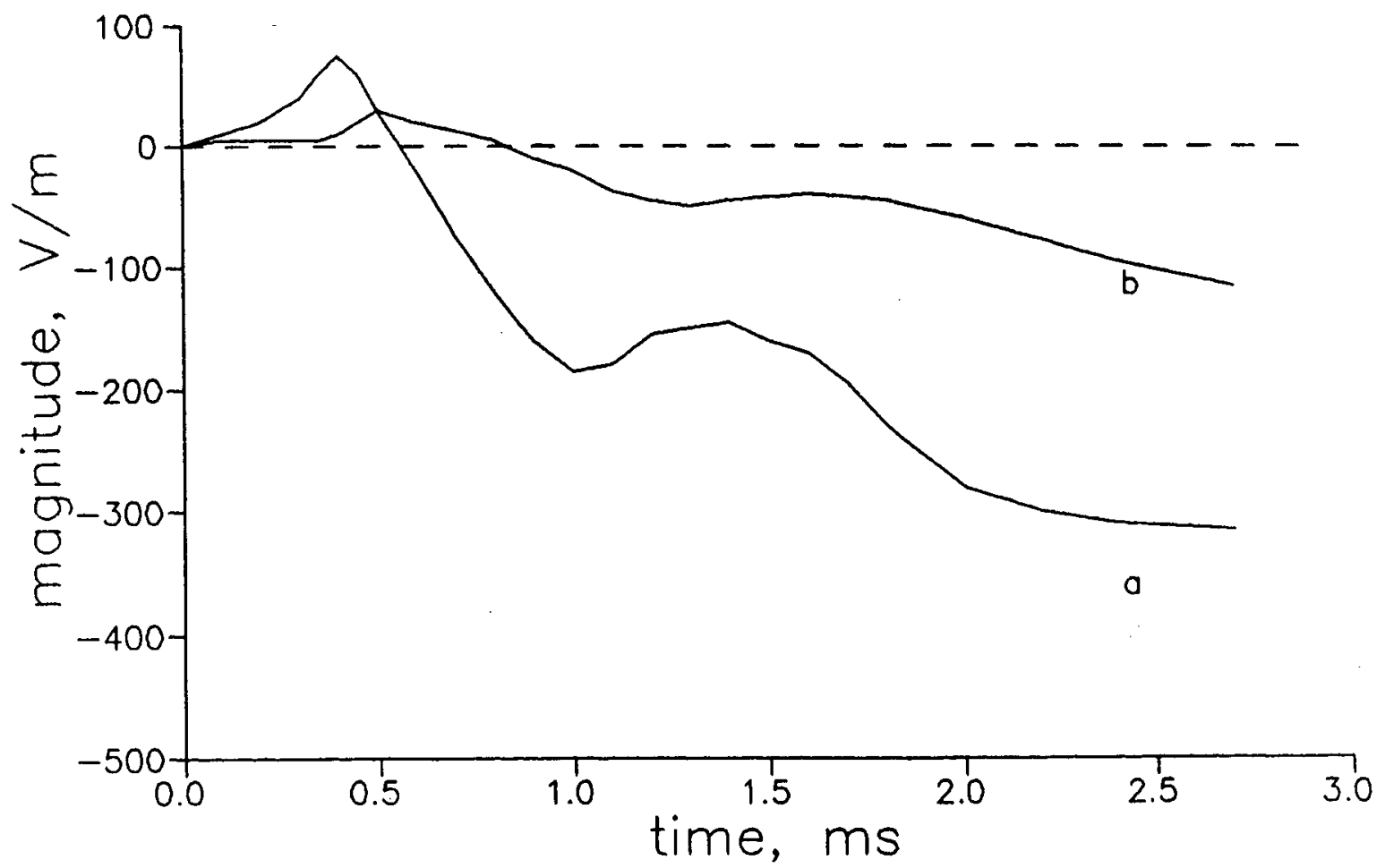

Figure 2.2. Typical waveform of electric field signals generated by the detonation of high explosive charges in air. (a) $-R=2.5 \mathrm{~m}$; (b) $-R=3.5 \mathrm{~m}$. [Boronin at al., 1973]. 
explosion products border. On the base of the analysis of the experimental data the authors conclude that the registered electric field has dipole character and the sources of this field are localized behind the air layer, heated at the shock wave iront.

In the formula (2.2) the electric field strength changes with distance as $R^{3}$. But selivanov et al., [1990] note, that the experimental dependence of the decrease of the electric field strength with distance gives a value, which is less than $R^{-3}$ (Figure $2.3 \mathrm{a}, \mathrm{b}$ ).

Boronin et al. [1973], suggest the qualitative description of the electric field origin after the explosion in the air. Solid particles, created during the explosives detonation, are charged at the expense of the electrokinetic effect during the motion relatively gaseous products of the detonation. Gaseous products of the detonation gain the volume electric charge with opposite sign. In the initial time moment the velocity of the large particles is less than the gas velocity, but then the velocity of the gaseous products decreases, and the solid particles outstrip them. Since the charge sign, gained by the particles, doesn't depend on the direction of the relative velocity of the particle and gas motion, the change of the resulted dipole moment sign takes place in the moment of overtaking by the gaseous products particles. Solid particles keep their charge after the overtaking relatively the cold detonation products. They get into the air, heated by the shock wave, which has higher conductivity. Then the solid particles lose their charge. This charge assembles on the surface, which separates the heated air and detonation products with low conductivity. Subsequently, the resulting dipole moment pulses in accordance with the pulsation of the detonation gaseous products, i.e. the form of the impulse of the low-frequency radiation correlates with the low of the motion of the detonation products boundary. 


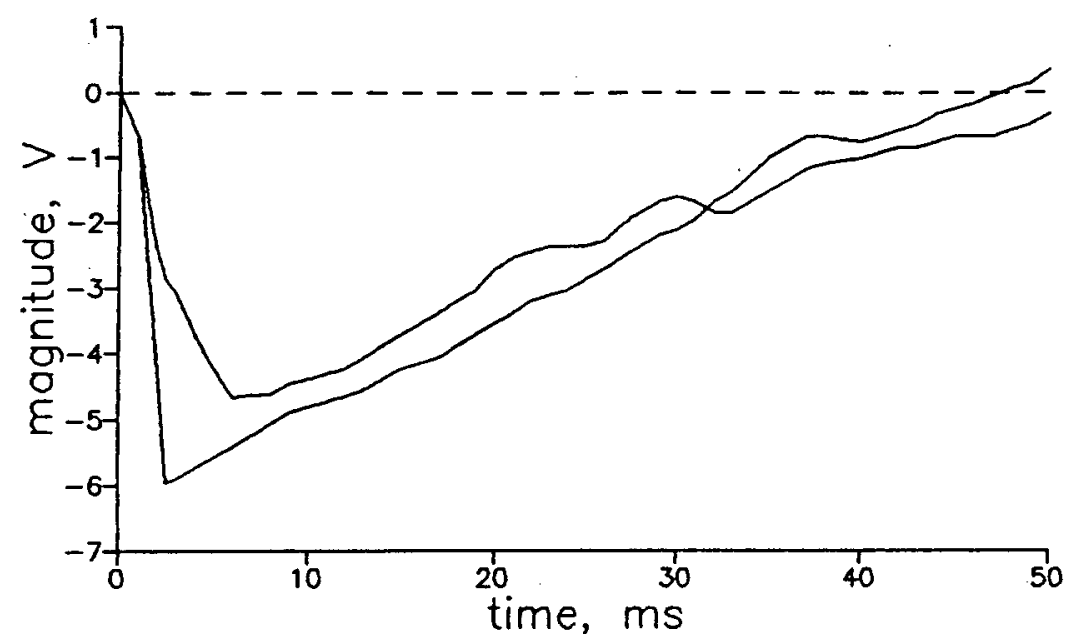

Figure 2.3. Typical electric field signols generoted by the detonation of high explosive charges at distance $R=2 \mathrm{~m}$ (two charges with mass $\left.C=0.04 \mathrm{~kg} ; E_{m}=1500 \mathrm{~V} / \mathrm{m}\right)$. [Selivanov at al.,1990].

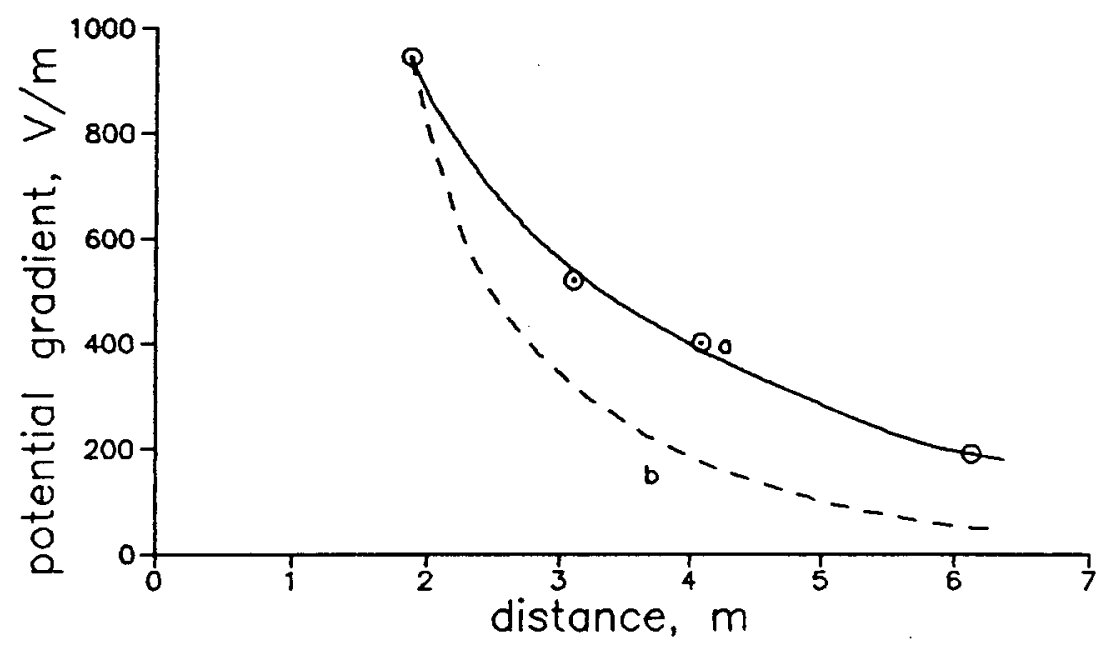

Figure 2.4. Magnitude of the electric field strength $E_{m}$ v.s. distance $R$. [Selivanov af al. 1990]
(a)- from experiment; (b) $-R^{-3}$ dependance. 
3. Electromagnetic signals generated by underground chemical and nuclear explosions.

\subsection{Electromagnetic emission generated by the nuclear explosions.}

A great deal of research has been devoted to the electromagnetic radiation from nuclear explosions in the atmosphere. Kompaneets [1959] was the first to propose a physical mechanism of electromagnetic radiation from nuclear explosions in the atmosphere. The gamma rays lose their energy by soattering Compton electrons from air molecules. Multiple scattering is ignored, and it is assumed that the compton electrons travel predominantly radially outward with mean energies of the order of $1 \mathrm{Mev}$. The air is ionized by the Compton electrons, which produce about $3 \cdot 10^{4}$ ion pairs per Mev. The outward Comton current is the mechanism of charge separation that produces an approximately radial electric field. The field, in turm, immediately establishes a conduction current tending to dissipate the field that produces it. If an electric dipole moment or any radiation observable at a distance is to be produced, there must be an asymmetry in the model.

For the air explosions the asymmetry appears because of the follow causes: The asymmetry of gamma rays emission [Kompaneets, 1959; Gilinsky, 1965]; Closeness to the Earth's surface [Sandmeir et al., 1972; Medvedev et al., 1967; Arsenin et al., 1981]; Heterogeneity of the atmosphere by density [Gilinsky and Peebles, 1968]; Presence of the Earth's electric and magnetic field [Karzas and Latter, 1962].

The electromagnetic radiation, generated by the nuclear explosion, has a wide frequency spectrum from a few hundreds of $\mathrm{MHz}$ to a few $\mathrm{KHz}$. Different components of gamma rays from the nuclear explosion, such as initial gamma rays, secondary gamma rays, and fission product gamma rays, take part in the compton mechanism of the generation of the electromagnetic radiation. The initial gamma rays give rise to the electromagnetic radiation in the high-frequency and low-frequency bands [Kompaneets, 1959; Gilinsky, 1965]. In parallel with initial 
gamma rays, the secondary gamma rays which generated by the inelastic scattering of last neutrons, give rise to the low-frequency electromagnetic radiation [Medvedev and Metelkin, 1979].

The current system, formed after the nuclear explosion, doesn't disappear at once and it is to produce a macroscopic separation of the electric charges in the cloud of the explosion products. Lighting, observed during the first milliseconds after the explosion, are evidence of it [Uman, 1972]. The generation of the electromagnetic radiation, connected with the quick transference of the electric charge from one part of the cloud formed by the explosion products to another, take place too.

The strongly heated plasma of the explosion (with initial temperature of about $1 \mathrm{KeV}$ ) expanded in the Earth's magnetic field, is the source of the electromagnetic radiation [Leipunsky, 1960; Kompaneets, 1977]. The conductivity currents are generated in plasma with high conductivity, which is about $10^{-3} \mathrm{~s} / \mathrm{m}$. It results in the "shielding" of the external magnetic field. "Extracting" of the geomagnetic field from the ionized area at the phase of the expensing and the following relaxation of the currents is the cause of the geomagnetic disturbances and the radio-emission of the magneto-dipole type [Karzas, 1962; Fedorovich, 1969].

The electromagnetic radiation from the underground explosion is expressed more weakly, than from the air explosion. The reduction of the electromagnetic radiation is conditioned by the shielding action of the conductivity layers of the Earth. The mechanism of the generation of the electromagnetic radiation by the explosions in the atmosphere are considered as possible mechanism of such generation by the underground explosions in the first place. The currents of the Compton electrons and the diamagnetic effect of plasma, expanded in the underground cavity, are these mechanisms [Zablocki, 1966; Sweeney, 1989]. The reduction of the electromagnetic radiation, corresponding to the compton mechanism, are the strongest in the conductive medium because of the high-frequency character of this mechanism. Besides that, we must take into account the electromagnetjc radiation, 
generated by the currents in the up-hole emplacement pipe. [Sweeney, 1989].

Ablyazor et al., [1988], calculated the effective magnetic moment of plasma in the underground cavity. It was shown, that the amplitude-temporal characteristics of the signals on the surface of the conductive medium depends on the plasma temperature, appropriatenesses of the cavity development, the environment conductivity and some other parameters.

However, we assume that there is one more mechanism, which to be able to contribute to the generation of the electromagnetic radiation from the underground explosion. It is the mechanism of the generation of the electromagnetic radiation due to the shock wave compression and fragmentation of solids.

From the experimental data it is known, that in some cases the polarity of the observed signals corresponds to the electric not magnetic dipole. Apparently, the effect of the shock polarization in the area of the strong shock wave becomes evident exactly in these cases.

In the next section we'll consider in detail the generation of the electromagnetic radiation by the shock compression of solids.

In the section 3.4 .5 we'll consider extra low-frequency electric field generarated by the underground nuclear tests in the air.

We monitored 21 large-scale explosions for electromagnetic signals from 1976 through 1991. These events are listed in Table 3.1. The underground nuclear tests were conducted at the Semipalatinsk Test Site (Degelen, Balapan) and at the Novay Zemlay Test Site (Matochkin). 
Table 3.1.

\begin{tabular}{|c|c|c|c|c|c|c|}
\hline $\begin{array}{l}\text { Event } \\
\text { No. }\end{array}$ & $\begin{array}{l}\text { Event } \\
\text { name }\end{array}$ & Date & $\begin{array}{l}\text { Time } \\
\text { UT }\end{array}$ & $\begin{array}{l}\text { Yield, kt } \\
\text { (mass, kg) }\end{array}$ & $\begin{array}{l}\text { Event } \\
\text { type }\end{array}$ & Location \\
\hline 1. & $A-14$ & 29.09 .76 & $03: 00$ & $120 \mathrm{kt}$ & tunnel & Matochkin \\
\hline 2. & $A-17$ & 01.09 .77 & $03: 00$ & $95 \mathrm{kt}$ & tunnel & Matochkin \\
\hline 3. & $A-18$ & 10.08 .78 & $08: 00$ & $15 \mathrm{kt}$ & tunnel & Matochkin \\
\hline 4. & $A-37$ & 11.10 .82 & $07: 15$ & $75 \mathrm{kt}$ & tunnel & chkin \\
\hline 5. & 215 & 30.05 .83 & $07: 34$ & $17 \mathrm{kt}$ & tunnel & \\
\hline 6. & hemical & 26.06 .83 & - & $2.6 \cdot 10^{6} \mathrm{~kg}$ & excavation & $\begin{array}{l}\text { Buchara, } \\
\text { Uzbekistan }\end{array}$ \\
\hline 7 . & $A-21$ & 25.09 .83 & $13: 10$ & $120 \mathrm{kt}$ & tunnel & Matochkin \\
\hline 8. & 190 & 15.04 .84 & $07: 17$ & $53 \mathrm{kt}$ & tunnel & Degelen \\
\hline 9. & 1316 & 25.04 .84 & - & $84 \mathrm{kt}$ & borehole & Balapan \\
\hline 10. & 1414 & 26.05 .84 & - & $143 \mathrm{kt}$ & borehole & Balapan \\
\hline 11. & $A-100$ & 26.08 .84 & - & & tunnel & Matochkin \\
\hline 12. & $1061_{\text {bis }}$ & 15.06 .85 & $04: 57$ & $\approx 3 \mathrm{kt}$ & borehole & Balapan \\
\hline 13. & 1341 & 15.06 .85 & $04: 57$ & $115 \mathrm{kt}$ & borehole. & Balapan \\
\hline 14. $\mathrm{ch}$ & hemical & 27.06 .85 & $11: 57$ & $5 \cdot 10^{5} \mathrm{~kg}$ & on surface & Degelen \\
\hline 15. & 1354 & 30.06 .85 & $06: 39$ & $108 \mathrm{kt}$ & borehole & Balapan \\
\hline 16. & 1326 & 20.06 .87 & - & $95 \mathrm{kt}$ & borehole & Balapan \\
\hline 17. ch & hemical & 29.06 .87 & $04: 55$ & $5.01 \cdot 10^{5} \mathrm{~kg}$ & on surface & Degelen \\
\hline 18. & 168 & 17.07 .87 & $05: 17$ & $79 \mathrm{kt}$ & tunnel & Degelen \\
\hline 19. ch & hemical & 25.08 .87 & $14: 00$ & $10^{6} \mathrm{~kg}$ & on surface & Matochkin \\
\hline 20. ch & $\begin{array}{l}\text { hemical } \\
\text { our expl }\end{array}$ & $\begin{array}{l}09.87 \\
\text { losions }\end{array}$ & - & $3.2 \cdot 10^{4} \mathrm{~kg}$ & excavation & $\begin{array}{l}\text { Kok-beI, } \\
\text { Kirgizia }\end{array}$ \\
\hline 1. ch & & & $10: 25$ & $9.01 \cdot 10^{5} \mathrm{~kg}$ & on surface & PIesetsk \\
\hline
\end{tabular}


3.2. Source modeling for low-frequency electromagnetic signals in the soil.

Dynamic deformation and destruction of solids is accompanied by various electromagnetic effects. Main appropriatenesses of the effects depend on the destmuction scale, intensity and duration of the influence and some other factors.

Such effects as the generation of low-frequency electromagnetic fields (with a frequency of $100 \mathrm{kHz}$ and under), emission of charged particles, luminescence, formation of micro discharges in cracks are observed in the laboratory experiments of the small-scale samples destruction. Fractions of the destroyed matter generally have an electric charges.

The experimental studies of shock compression of various matters, such as metals, semi-conductors, dielectrics, show that always there is a sudden changes of the potential at the shock wave front (i.e. there is a potential difference between two points separated by a shock wave front). Effects of the shock magnetization and demagnetization of matter arise in the magnetic materials.

These effects determine the features of electromagnetic fields formed by large-scale tectonic effects, such as earthquakes, volcanos eruption, underground explosions with large energy yields. So, it is expedient first to study microscopic processes, which are the base of the mentioned effects.

3.2.1 Effects of shock polarization and depolarization of solid dielectrics

Researches made in this field began from the work of Stepanov, 1933, where it was founded, that potential difference arises between the opposite sides of the deformed samples during slow deformation of ionic crystals. Stepanov's effect can't be explained by known effects of pyro- and piezoelectric nature. In some papers [Caffin,1955; Fishbach,1958; урусовская,1968] it was shown, that the cause of this effect in ionic crystal is conditioned by the motion of electric charged 
dislocations under the influence of mechanical stresses in the crystals.

Besides that, analogous effect is observed during the shock wave spreading in dielectrics. The principle scheme of the typical laboratory experiment [Mineev, 1976] is shown at Figure 3.2.1. The test sample 1 was pressed between the electrode 2 and the metallic screen 4. Direction of the flat shock wave front motion is marked by arrows. The electrode and the protective ring were made from metals with acoustic impedance close to the impedance of the sample material. Letters $R$ and $R_{1}$ represent input resistance if the oscillograph and the resistance of the protective ring loading. The circuit parameters choice was realized to receive the actual equivalence of the mentioned scheme to the electric circuit of the short-closed condenser formed by the screen and the electrode.

The current in the external circuit, which doesn't contain any voltage sources, arose once the shock wave travels into the sample. That is to say that the e.m.f. (electro-motive forces) or a sudden change of the potential at the shook front is generated. Voltage drop at the resistance $\mathrm{R}$ of the load was registered in the experiments. During the experiment it was found that the polarized current was directly proportional to the electrode square $S$. So, the square of the protective ring $S_{1}$ and loading resistance $R_{1}$ were chosen so that the equation $S_{1} R_{1}=S R$ will be true. In this case the voltage drops at the resistances $R_{1}$ and $R$ are the same, and it allows to avoid the influence of the border effects. Typical values of the parameters are the next: $S_{1}=S=1-3 \mathrm{~cm}^{2}$, the sample thickness is $l=0.1-3 \mathrm{~cm}$, and $R_{1}=R=92 \mathrm{Om}$. They provided the truth of the short-closed electric current conditions: $t_{r} \ll \tau / U$, where $t_{r}$ is time of the measuring circuit relaxation, and $U$ is the shock wave velocity.

Figure 3.2.2 presents typical record of the polarized current generated by the shock loading of the monocrystal $\mathrm{NaCl}$ with pressure amplitude of $10 \mathrm{GPa}$ [Mineev, 1976]. The vertical axis represents the voltage drop at the resistance of the load. The current arise at the moment of the shock wave entrance into the sample, and it sudden decreases when the wave go out from 


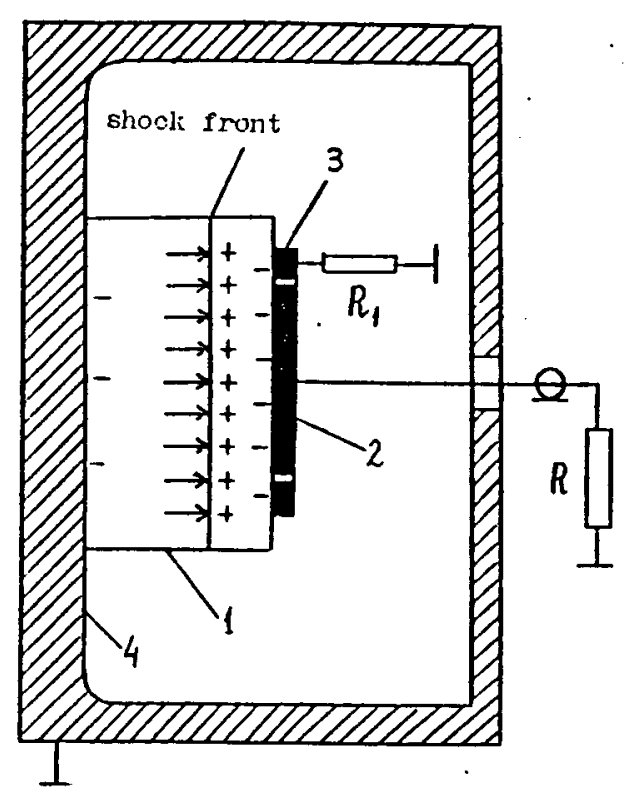

Figure 3.2.1. The prinoiple scheme of the typical laboratory experiment [Mineev, 1976].

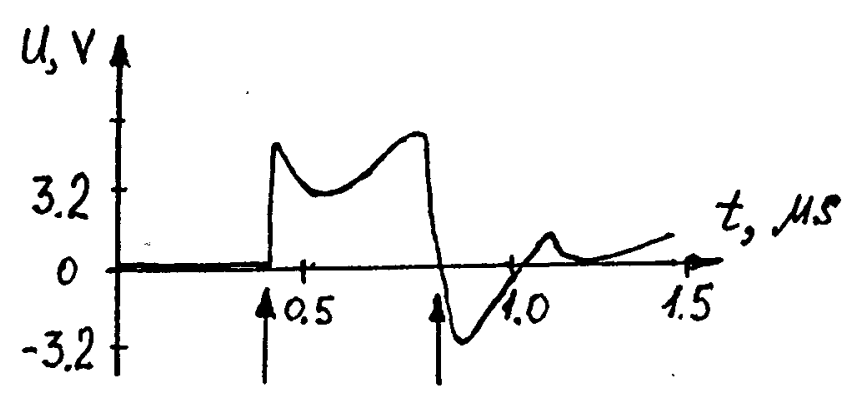

Figure 3.2.2. Typical record of the polarized current generated by the shock loading of the monocrystal $\mathrm{NaCl}$ with pressure amplitude of $10 \mathrm{GPa}$ [Mineev, 1976]. 
the sample. Appropriate time moments are marked at Figure 3.2.2 by arrows.

The rise time of the signal is equal to $0.02-0.10 \mu \mathrm{s}$. Generally, by the authors opinion, it is conditioned by the unsimultaneity of the shock wave entrance into the different parts of the sample. Effective density of the surface charge at the shock wave front was equal to $10^{-4}-10^{-3} \mathrm{C} / \mathrm{m}^{2}$ in dependence on the crystalographic direction of the shock wave spreading. Time of the polarization relaxation was $0.1-0.2 \mu \mathrm{s}$.

Mentioned appropriatenesses are typical for all investigated ionic crystals ( $\mathrm{NaCl}, \mathrm{LiF}, \mathrm{KBr}, \mathrm{RbCl}, \mathrm{MgO}, \mathrm{LiD}$, $\mathrm{CsCl}$ and so on). The features of one or another signals depend on the shock wave amplitude, characteristics of the atomic structure of the investigated material, concentration and kind of the alloying admixtures, density of the dislocations in the sample and some other parameters [Ivanov, 1965; Iinde, 1966].

Effect of the shock polarization was founded in many polar dielectrics, such as polymethylmetacrylate, polyehtylene, trinitrotoluene, polyamide pitch, water and in semi-conductors of $p$ - and n-types (silicon, germanium and so on). For these materials the effective surface density of the charge at the shock wave front varies in the range of $10^{-7}-10^{-2} \mathrm{c} / \mathrm{m}^{2}$, and relaxation time is about 0.1-1 $\mathrm{Hs}$ [Eichelberger, 1962; Mineev, $1967]$.

In polar dielectrics the physical mechanism of this effect depends on the orientation of the polar molecules in the direction of the shock wave motion. Turn of the molecule under the influence of the mechanical stresses in the wave can occur when the mass of one part of the molecule is greater then the mass of the other part. As result, the element of the medium volume in the shock wave gains an inducted dipole moment, i.e. a polarization. Thermal motion of the molecules exerts disorienting influence and results in the relaxation of the shock polarization [Eichelberger, 1962]. Some dielectrics acquire conductor properties behind the shock wave front. It results in the decrease of the shock polarization too. In this case the polarized charges are screened by the current bearers. Reverse effect, i.e. shock depolarization, was observed in the polarized segnetoelectrics and 
piezoelectrics [Neilson,1957;1960]. It was founded, that this effect is conditioned by the partial or full loss of the segnetoelectric properties of the material after its shook compression.

For ionic crystals with simple atomic structure such as $\mathrm{NaCl}$, it was supposed the explanation of the shock polarization effect based on the relative elastic linear displacement of the ions with different signs in the shook wave [Harris, 1965]. Because of the slight elastic displacements of the ions after small shock pressures, the conclusions of this theory [Harris, 1965] don't conform to the experience [Mineev, 1967] neither by the amplitude, nor by the duration of the signal. Early it was noted [Linde, 1966; Wong, 1969] that the possible cause of the effect might be connected with the presence of the electric charged dislocation (old) and compensating clouds of the point defects in the crystals. Investigation of the ionic crystals led to result, which, in some authors view [Tunaev, 1968; 1971], contradicts this viewpoint, because the amplitude of the polarized current decreased with increase of the investigated materials plasticity, and the polarization sign in the area of small pressures didn't correspond to the sign of the charges of new as well as old dislocations.

Mineev [1976; 1967] supposed, that the effect of the shock polarization in the ionic crystals is conditioned by the point defects, which diffuse through the shock wave front. Under the low pressures (up to 40-50 GPa) the positive charged vacancies, which are the current bearers under the normal condition, were the most important. From the data [Pratt, 1961; Klein, 1965] the concentration of the point defects in the shock reaches $10^{16}-10^{17} \mathrm{~cm}^{-3}$ per each percent of the plastic deformation. Also, it was founded, that signals amplitudes depend on the initial concentration of alloying admixtures $\left(\mathrm{Ca}^{2+}, \mathrm{Mn}^{2+}, \mathrm{I}^{-}\right.$and so on) [Tunaev, 1969; 1973]. But the quantitative estimations didn't coincide with the experiment due to the small coeffioients of the defects diffusion.

The explanation of the effect by the influence of the crystal destmiction and the motion of the charged boundaries between blocks isn't in agreement with the laboratory experiments, because the time of the polarization increase from 
this mechanism is in a few orders greater than the experimental value. Also, it was noted, that the shock polarization in ionic crystals is impossible at the electronic level, because the time of the electronic relaxation (of about $10^{-14} \mathrm{~s}$ for connected electrons and of about $10^{-8} \mathrm{~s}$ for free electrons) is too small.

In the investigations mentioned above the fact, that the electric charge of the movable border dislocations can have different signs during slow and quick deformation, wasn't taken into account. The value of this charge is determined by the difference of the connection energies for the dislocations of the vacancies of the negative and positive ions [K]ein, 1966; Shevtsova, 1983; Tyapunina, 1988]. So, in future, in theoretical analysis of the shock polarization effects we'll consider the point defects as well as the dislocations.

Let's consider the results of Surkov [1985, 1992] and Sirotkin, [1986] based on the study of the kinetios of the point and Iinear struoture defects at the area of the shock wave front. Advantage of this approach is that the dependence of the diffusion coefficients and the velocity of the defects reproduction from the shock wave parameters and lattice structure to take into account.

Let's take a look at the one-dimensional compression of the dielectric in the flat shook wave with assumption, that the wave front has finite thickness. At first we'II study the processes connected with the charge transfer by point defects. There are intensive reproduction of the Frenkel defects (pairs of vacancy and interknot ion) at the shock wave front. The compression of the crystal lattice results in the distortion of the balanced configuration of the atoms near the defects, so the last can move. Let's use the next convenient signs: $N_{i 1}$ and $\mathrm{N}_{\mathrm{i} 2}$ to designate the concentration of the particles of kind $i$ at the cross-sections with coordinates $(x-\delta / 2)$ and $(x+\delta / 2)$ correspondingly ( $\delta$ is the lattice's constant), and $\nu_{i}^{ \pm}$for the designation of the frequencies of particle jump over one interatomic distance, where the indexes + and - correspond to the defects displacement in the direction of the wave spreading and in the opposite direction [Malkovich, 1982]. Then, the projection of the flow density is $j_{i x}=\left(\nu_{i 1}^{+} N_{i 1}-v_{i 2}^{-} N_{i 2}\right) \delta$. If 
we'll expand this equation on the parameter $\delta$ and consider the transfer of the defects together with the matter and under the influence of the electric field, then

$$
j_{i x}=N_{i} \delta\left[v_{i}^{+}-v_{i}-\frac{\delta}{2} \frac{\partial}{\partial x}\left(v_{i}^{+}+v_{i}\right)\right]-\frac{\delta^{2}}{2}\left(\nu_{i}^{+}+v_{i}\right) \frac{\partial N_{i}}{\partial x}+N_{i} v_{x}+\frac{\sigma_{i} E_{x}}{q_{i}} \text {. }
$$

In this formula $v_{x}$ is the projection of the matter mass velocity $\vec{v}$ at the shock wave, $q_{i}$ is the charge of the particles of this kind, $\sigma_{i}$ is the ionic electrical conductivity, and $\mathrm{E}_{x}$ is the component of the electric field strength. Functions $\sigma_{i}^{x}$ and $\nu_{i}^{ \pm}$depend on the temperature $T$ and mechanical stresses in the shock wave. As we can see from the analysis, the differences of the functions $\nu^{+}$and $\nu^{-}$doesn't quantitatively influence on the investigated effect. So, subsequently we'll consider, that $\nu^{+}=\nu^{-} \equiv \nu$.

The speed of the defects reproduction during the plastic deformation of the medium is proportional to the velocity of the change of the tensor deviator of the mechanical stresses $\dot{\gamma}$. Taking the reproduction processes and recombinations of the defects into account and extrapolating this conclusions (3.2.1) at the three-dimensional case, we can write the equation of the continuity and Maxwell's equations as

$\vec{J}_{i}=-\delta^{2} \nabla \nu_{i} N_{i}+N_{i} \vec{v}+\frac{\sigma_{i} \vec{E}}{q_{i}}$,

$\overline{\partial t} \bar{N}_{i}+\nabla \vec{J}_{i}=M_{i} \dot{\gamma}-\mu_{n i} N_{n} N_{i}$,

$\varepsilon_{0} \nabla \varepsilon \vec{E}=\rho, \quad \rho=\sum_{i} q_{i}\left(N_{i}-N_{i 0}\right)$,

where $\varepsilon$ - dielectric permittivity, $\varepsilon_{0}$ - dielectric permittivity of Iree space, $\mathrm{N}_{i_{0}}$ - initial defects concentration, $\rho$ electric charge density, $M_{i}-$ coefficient of the defects reproduction, and $\mu_{n i}$ coefficient of the recombination of the vacancies and interknot ions, $\mathbb{M}_{n}=M_{i}$ and $\mu_{n i}=\mu_{i n}$ from the law of the electric charge conservation.

Coefficients $\mu_{n i}$ are proportional to the cross-section of the scattering of the particles of order $4 \delta^{2}$, and the particles velocity are of order $\nu \delta$, i.e. $\mu_{n i} \approx \nu \delta^{3}$. The estimations with typical values of the parameters in the shock $M_{i} \approx 10^{25} \mathrm{~m}^{-3}, \quad \dot{\gamma}=10^{7} \mathrm{c}^{-1}, \quad N_{i} \approx 10^{24} \mathrm{~m}^{-3}, \quad \delta \approx 0.281 \mathrm{mum}$ (millimicron) (for the lattice of $\mathrm{NaCl}$ ) show that the 
recombination term is small as compared with the source function $M \dot{\gamma}$ up to the frequencies $v \approx 10^{12} \mathrm{~Hz}$. So, subsequently, this term isn't considered. On condition that $e \delta^{2}\left|\nabla \nu_{i} N_{i}\right|>\sigma_{i} E$ (e - elementary charge) we can neglect the conductivity. If we'll use the equation:

$$
\sigma_{i}=e^{2} \delta^{2} N_{i}\left(\nu_{i}^{+}+\nu_{i}^{-}\right) /(2 k T)
$$

where $\mathrm{k}$ is Boltzmann constant, than the mentioned limitation is $\mathrm{E}<\mathrm{kT} /(\mathrm{e} \lambda)$, where $\lambda$ is the characteristic size of the shock wave in the crystals. When $\mathrm{T} \times 10^{3} \mathrm{~K}$ and $\lambda \approx 10^{-7}-10^{-6} \mathrm{~m}$ we'll receive the next result $\mathrm{E}<10^{5}-10^{6} \mathrm{~V} / \mathrm{m}$.

So, at the shock wave front, where gradients of all values are great, we can to neglect the relaxation processes, connected with the particles recombination and the influence of the medium conductivity. In this case the particles behavior is described by independent equations, which might be received by using of $(3.2 .2)$ in the equation (3.2.3). Without the index $i$ we can write the follow equation for the flat shock wave, spreading along $\mathrm{X}$-axis:

$\frac{\partial N}{\partial t}+\frac{\partial N \nabla}{\partial x}-\delta^{2} \frac{\partial^{2} \nu N}{\partial x^{2}}=M \dot{\gamma}$

where $\dot{\gamma}$ is the modulus of the displacement plastic deformation. The equation (3.2.5) is the equation of Fokker-Plank kind with the source. With constant $v$ the last item in the left part of the equation (3.2.5) transforms to the common form, typical for diffusion equations with diffusion coefficient $v \delta^{2}$.

In the case of the charge transfer by border dislocations, letters $N$ and $M$ determine the number of the dislocation and coefficient of their reproduction with respect to the square unit, and $q$ determines electric charge per unit of the dislocation length, which is constant. [Shevtsova, 1983]. The frequency is $v=c_{d} / \delta$, where $c_{d}$ is the velocity of the dislocation slip. With the consideration of the mentioned changes the equation (3.2.5) is available to describe the dislocations kinetics.

Sirotkin, [1986] found the solution of the equation (3.2.5) for the stationary shock wave with parameters, which depend on the single variable $\xi=(x-U t) / \lambda$, where $U$ is the shock wave velocity, $\lambda$ is characteristic scale of the shock wave. This solution, received by the exception on small 
parameter $\alpha=\left(\delta^{2} \nu_{0}\right) /(U \lambda)$, is

$\mathrm{N}-\mathrm{N}_{0}=\mathrm{M} \gamma-a \frac{\mathrm{d}}{\mathrm{d} \xi} \frac{\nu}{\nu_{0}}\left(\mathrm{~N}_{0}+\mathrm{M} \gamma\right)$,

where $N_{0}$ and $\nu_{0}$ are the values of the concentration and frequency of defects jumps before the shock wave front when $\xi \rightarrow \infty$. At zero approximation $(\alpha=0)$, the concentration change $N-N_{0}$ is conditioned only by the defects reproduction. At the next approach by $\alpha$ the concentration change provoked by the displacement of the defects with frequency $v$ is taken into account.

Let's analyze the features of the solution (3.2.6) for low voltage in the shock wave, when point defects and dislocations move on the base of thermofluctuated mechanism. The frequency of the jumps of the defects of order $i$ from one balanced position to another is determined by the follow equation:

$\nu_{i}=\nu_{i^{+}} \exp \left[-u_{i} /(k T)\right], \quad u_{i}=u_{0 i}-\beta_{i} \gamma$.

where $k$ is Boltzmann constant, $u_{i}$ is the activation energy, $\beta_{i}$ is stretch parameter. Linear relation between $u_{i}$ and plastic deformation $\gamma$ is true for $\gamma$ « 1, i.e. when the influence of the unharmonism of the lattice oscillations is insignificant.

Parameters of the equation (3.2.7) might be estimated in the case of the crystal lattice of $\mathrm{NaCl}$ kind, for which we know [Kittel, 1978] the empiric formulas for the energy of the ions interaction. Because the radius of ions $\mathrm{Cl}^{-}$is greater than the radius of ions $\mathrm{Na}^{+}$, the activation energy of the last is smaller. During the compression in the crystalographic direction $[1,0,0]$ we receive the follow estimations [Surkov, 1985; Sirotkin, 1986]: for cations $\mathrm{Na}^{+} u_{0 k}=3,2-4,5 \mathrm{eV}$, $\beta_{k}=7,1-11 \mathrm{eV}$; for vacancies $\mathrm{Na}^{+} u_{0 \mathrm{v}}=0,86 \mathrm{eV}, \beta_{\mathrm{v}}=-1,5 \mathrm{eV}$. Due to the different signs of $\beta_{k}$ and $\beta_{v}$ the behavior of the cations and vacancies in the shook wave are not the same, because the growth of the shock pressure and deformation $\gamma$ is accompanied by the increase of the frequency $v_{k}$ and decrease of the frequency $v_{v}$.

Assuming that the charge distribution is determined by the displacement of the defects of the same kind, we can calculate the density of the electric charge $\rho(\xi)$ and the 
projection of the electric field voltage $E_{x}(\xi)$ at the shock wave front with help of $(3.2 .4),(3.2 .6)$, and $(3.2 .7)$.

$$
\begin{aligned}
& \rho=\frac{q \delta^{2}}{\lambda U} \frac{d}{d \xi} \nu(\xi)\left[N_{0}+M \gamma(\xi)\right] ; \\
& E_{x}=\frac{q \delta^{2}}{\varepsilon \varepsilon_{0} U}\left[\nu_{0} N_{0}-\nu(\xi)\left\{N_{0}+M \gamma(\xi)\right\}\right],
\end{aligned}
$$

where $\nu=\nu \cdot \exp \left[-\left\{u_{0}-\beta \gamma(\xi)\right\} /(k T)\right], \nu_{0}=\nu, \exp \left[-u_{0} /(k T)\right]$.

Let's approximate the deformation at the shock wave front by the follow dependence: $\gamma=\left(\gamma_{m} / 2\right)(1-\operatorname{th} \xi)$, where $\gamma_{\mathrm{m}}$ is the amplitude of the shock compression. Besides that, we'll use the next data: $\varepsilon=5.3, \quad \lambda=0.1 \mu \mathrm{m}, \nu_{*}=5 \cdot 10^{12} \mathrm{~Hz}, \quad M=10^{25} \mathrm{~m}^{-3}$, $\mathrm{N}_{0}=10^{22} \mathrm{~m}^{-3}, \delta=0.3 \mathrm{mum}$, and also the empiric parameters of the shock compressed $\mathrm{NaCl}$ under the pressure of $10 \mathrm{GPa}$ [Baum, 1975]: $U=4.4 \mathrm{~km} / \mathrm{s}, \mathrm{T}=450 \mathrm{~K} ., \gamma_{\mathrm{m}}=0,22$. The calculations by the formula (3.2.8) show that the maximum density of the cations charge is $\rho+1.2 \cdot 10^{-16} \mathrm{c} / \mathrm{m}^{3}$ and of the anions charge is p $4,4 \cdot 10^{-9} \mathrm{c} / \mathrm{m}^{3}$, the charge density being positive in the both cases (it means the abundance of the cations and the shortage of the anions at the shock wave). Also, the electric field voltage is not grater $0,1 \mu \mathrm{V} / \mathrm{m}$. It means, that the thermofluctuation mechanism of the defects displacement isn't in agreement with the experimental data [Mineev, 1976].

Other mechanism of the effect is possible, when it is achieved the threshold deformation $\gamma_{*}=u_{0 k} / \beta_{k} \approx 0,4$ and there is above-barrier displacement of the cations. Real value of $\gamma$. might be less than this value, because of the influence of the extension of the atoms nearest to the cation and at the expense of the unharmonism of the crystal lattice oscillations, which weren't taken into account in works of Syrkov, [1985] and Sirotkin, [1986]. Apparently, the vacancies importance decrease with the pressure growth because of the increase of the potential barrier $u_{v}$. When we'll consider only the motion of the cations with frequencies $\nu=\nu_{*}=$ const, we'Il receive the next relations:

$$
\begin{aligned}
& \rho=-\frac{q \delta^{2} \nu_{0} M}{\lambda U} \frac{d \gamma}{d \xi}, \quad E_{x}=-\frac{q \delta^{2} v_{0} M}{\varepsilon \varepsilon_{0} U}\left[\gamma(\xi)-\gamma_{0}\right]+E_{0} ; \\
& E_{0}=-\frac{q \delta^{2}}{\varepsilon \varepsilon_{0} U}\left[\nu_{*}\left(N_{0}+M \gamma\right)-\nu_{0} N_{0}\right] .
\end{aligned}
$$


For dislocations the charges $q_{d}$ per the length unit might be different at thermofluctuated stage (3.2.8) and at the slip phase (3.2.9). The threshold deformation for dislocations is $\gamma_{*}=Y / K$, where $Y$ is the fluidity limit and $K$ is the module of the volume compression. In the case of $\mathrm{NaCl}$ we have $\gamma \approx(3-6) \cdot 10^{-3}$, that is significantly less than the corresponding value for the cations.

From the equation (3.2.9) we can see, that the positive charge is concentrated at the shock front. With above values of the cations parameters we receive the follow estimations, typical for electric field in the shock wave for above-barrier regime of the defects displacement: $0.3 .6 \cdot 10^{2} \mathrm{c} / \mathrm{m}^{3}$, E $7.7 \cdot 10^{5} \mathrm{~V} / \mathrm{m}$. For dislocations, when $q_{d}=1.7 \cdot 10^{-11} \mathrm{C} / \mathrm{m}$ [Shevtsova, 1983] and $\mathbb{M}^{\sim 10^{17} \mathrm{~m}^{-2}}$ we receive, correspondingly, $p \approx 3.8 \cdot 10^{2} \mathrm{C} / \mathrm{m}^{3}$ and $\mathrm{E} \sim 8.2 \cdot 10^{5} \mathrm{~V} / \mathrm{m}$. The charges with different signs are located at the relaxation length or at the sample wall. These charges distribution might be received with consideration of the matter conductivity.

Mineev, [1976] measured the effective density of the charge $\Sigma$ per the unit of the square of the shock wave front surface.

$\Sigma=2 \varepsilon \varepsilon_{0} \mathrm{E}_{*} \gamma_{\mathrm{m}}=\frac{2 \mathrm{q} \delta^{2} \nu \cdot M \gamma_{\mathrm{m}}}{U}$,

The results of the experiments with $\mathrm{NaCl}$ for various crystalographic directions along which the shock wave was traveled, are represented at Figure 3.2 .3 by circles. Solid lines at this figure show the calculated dependences of $\Sigma$ (3.2.10) from the amplitude of the shock compression $\gamma_{\text {m. }}$.

As we can see from Figure 3.2.3 and formulas, mentioned above, the linear part of the dependence $\Sigma\left(\gamma_{m}\right)$ is explained by linear connection between the velocity of the dislocations reproduction (or point defects reproduction) and the velocity of the plastic deformation. The choice of the parameter $M$ results in the correlation of these data with data, received from the experiment. The results are shown in Table. In the case of the dislocation mechanism, as well as for the motion of the point defects, the calculated coefficients of the reproduction of the dislocations $M_{d}$ and point defects $M_{p}$ are close to the experimental values [Grigoriev,1979; Pierce,1961]. 

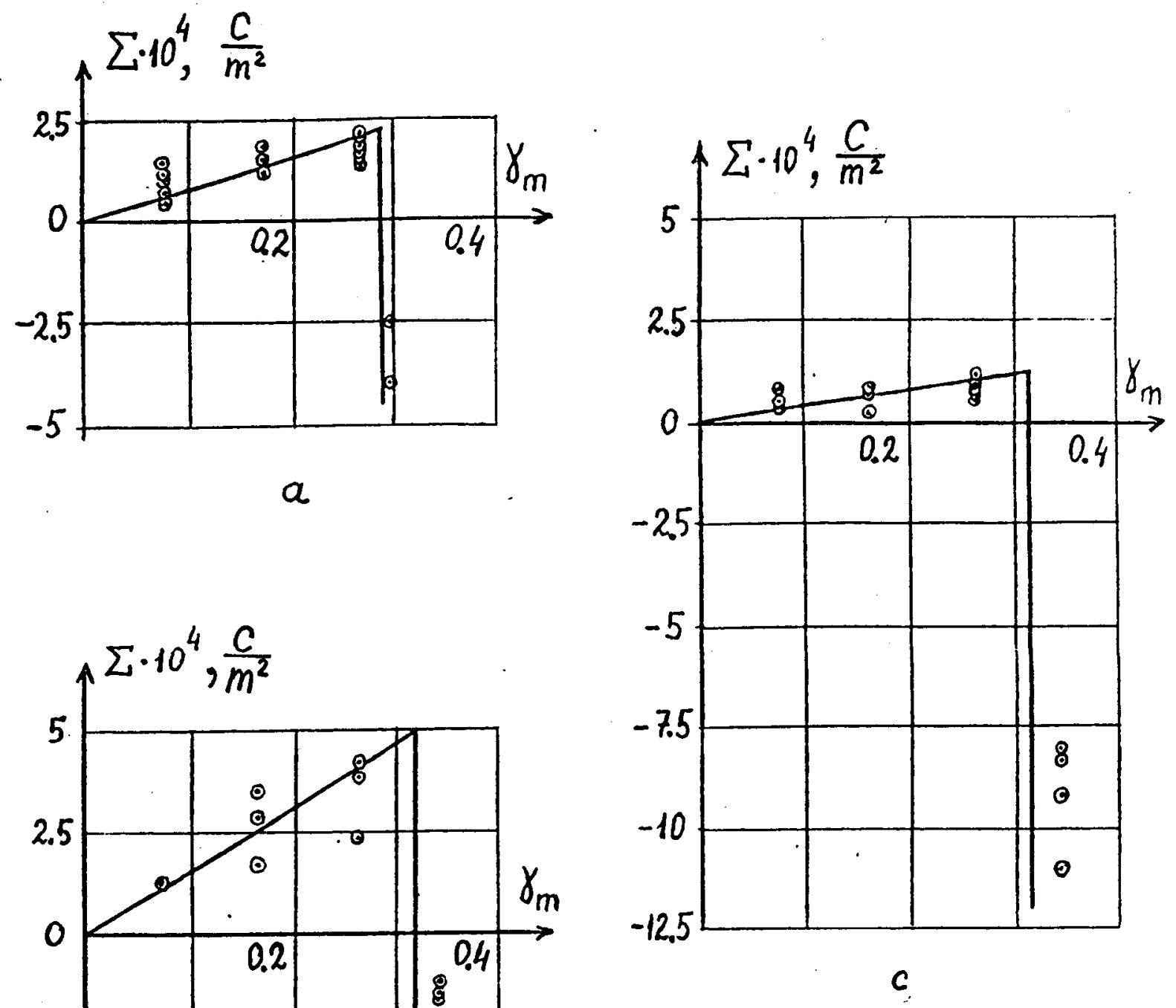

Figure 3.2.3. Magnitude of $\Sigma$ v.s. magnitude of the shock compression $\gamma_{\mathrm{m}}$ for various crystalographic directions: (a) $[1,0,0]$. (b) $[1,1,0]$. (c) $[1,1,1]$. Solid lines show the calculated dependences of $\Sigma(3.2 .10)$. 
Table 3.2.1.

\begin{tabular}{|c|c|c|c|c|}
\hline Direction & $M_{d}, 10^{17} \mathrm{~m}^{-2}$ & $M_{D}, 10^{25} \mathrm{~m}^{-3}$ & $\mathrm{E}_{+}, 10^{6} \mathrm{~V} / \mathrm{m}$ & $\gamma_{*}$ \\
\hline$[1,0,0]$ & 4.5 & 4.7 & 4.7 & 0.29 \\
\hline$[1,1,0]$ & 8.4 & 8.5 & 9.5 & 0.32 \\
\hline$[1,1,1]$ & 2.3 & 2.4 & 2.6 & 0.31 \\
\hline
\end{tabular}

Nevertheless, the dislocation mechanism of the shock polarization effect if more preferable, because the threshold of the effect by the deformation $\gamma$. for the dislocations is at 2-3 orders less. Considering that the experimental parameters $M_{d}$ and $M_{p}$ are connected by the ratio of $M_{d} q_{d}=e M_{p}$, we can assume, that the point defects are captured and transferred by the dislocations, and this process determines the charge of the last.

Above analysis isn't true under high pressures, when it is observed the change of the electric signal polarity. This effect might be connected with the fact, that for the point defects the threshold value is $\gamma_{*} \sim 0,3-0,4$. After the achievement of this value the velocity of their motion in the shock wave is comparable with the dislocations velocity, and sometimes it is greater. Change of the mechanism of the charge transfer will be rather sharp because of the exponential character of the dependences (3.8), and it can explain the threshold character of the effect.

In Table 3.2.1.we can see maximum values of $E_{*}$ with deformations $\gamma_{\mathrm{m}}$ corresponding to the fracture points at Figure 3.2.3. These values are at $1-2$ orders less the value of the critical field $E_{c}$ of the dielectric breakdown $\left(E_{c} \approx 1,3 \cdot 10^{8}\right.$ $\mathrm{V} / \mathrm{m}$ for $\mathrm{NaCl})$. But it is necessary to take into account the fact, that the increase of the defects concentration in the shock wave and significant deformation of the lattice with $\gamma \approx 0,3$ can result in the formation of the additional local levels in the prohibited zone of the crystal, i.e. decrease of $\mathrm{E}$. So, to explain the signal we can't neglect the effect of intrinsic breakdown of the crystal.

Phenomenological models, where it was assumed the presence 
of the sudden change of the polarization at the shock wave front, and the exponential relaxation of the shock polarization behind the front, were developed in some works. The relaxation was conditioned by the thermal and mechanical processes [AIIison, 1965], or by the electroconductivity, which arises in the dielectric behind the wave front [Ivanov, 1966; 1968; Zeldovich, 1967; Zaidel, 1968]. Mineev [1970], proposed an additional parameter, which were time of the increase of the matter shock polarization. Within the limits of this model some authors [Mineev, 1976; 1970; Allison, 1965; Ivanov, 1966; 1968; Zeldovich, 1967; Zaidel, 1968; Yakushev, 1968; Lisitsyn, 1970] calculated a polarizable current and compared them with the experimental data in order to find initial parameters of the models.

Let's estimate the polarizable current in the circuit of the short-closed condenser within the limits of the phenomenological method of approach [Zeldovich, 1967]. If we neglect the relaxation processes in the dielectric and the conductivity conditioned by the shock wave, the eleotric charge will be concentrated at the shock wave front, considered as infinitely narrow, and at the condenser plates (see Figure $3.2 .4)$.

Let's designate the surface density of the right plate charge as $\Sigma_{1}$, of the left plate charge as $\Sigma_{2}$, the dielectric permittivity of the medium before and behind the shock wave as $\varepsilon_{1}$ and $\varepsilon_{2}$ correspondingly. For lack of the load resistance in the extermal circuit the potential difference between the condenser plates is equal to zero, so

$$
\frac{\Sigma-\Sigma_{1}+\Sigma_{2}}{2 \varepsilon_{2} \varepsilon_{0}} \cdot\left(\frac{x}{k}\right)+\frac{\Sigma_{2}-\Sigma_{1}-\Sigma}{2 \varepsilon_{1} \varepsilon_{0}} \cdot(1-x)=0 \text {. }
$$

where $\Sigma$ is the density of the surface charge at the shock wave, $l$ is the dielectric thickness, $x=U \cdot t$ is the distance, passed by the shock wave, and $k=1+\gamma_{\text {is }}$ a degree of the matter compression in the shock wave. Taking into consideration the next ratio $\Sigma=\Sigma_{1}+\Sigma_{2}$ we find, that the current density in external circuit is described by the following law [Zeldovich, 1967]: 


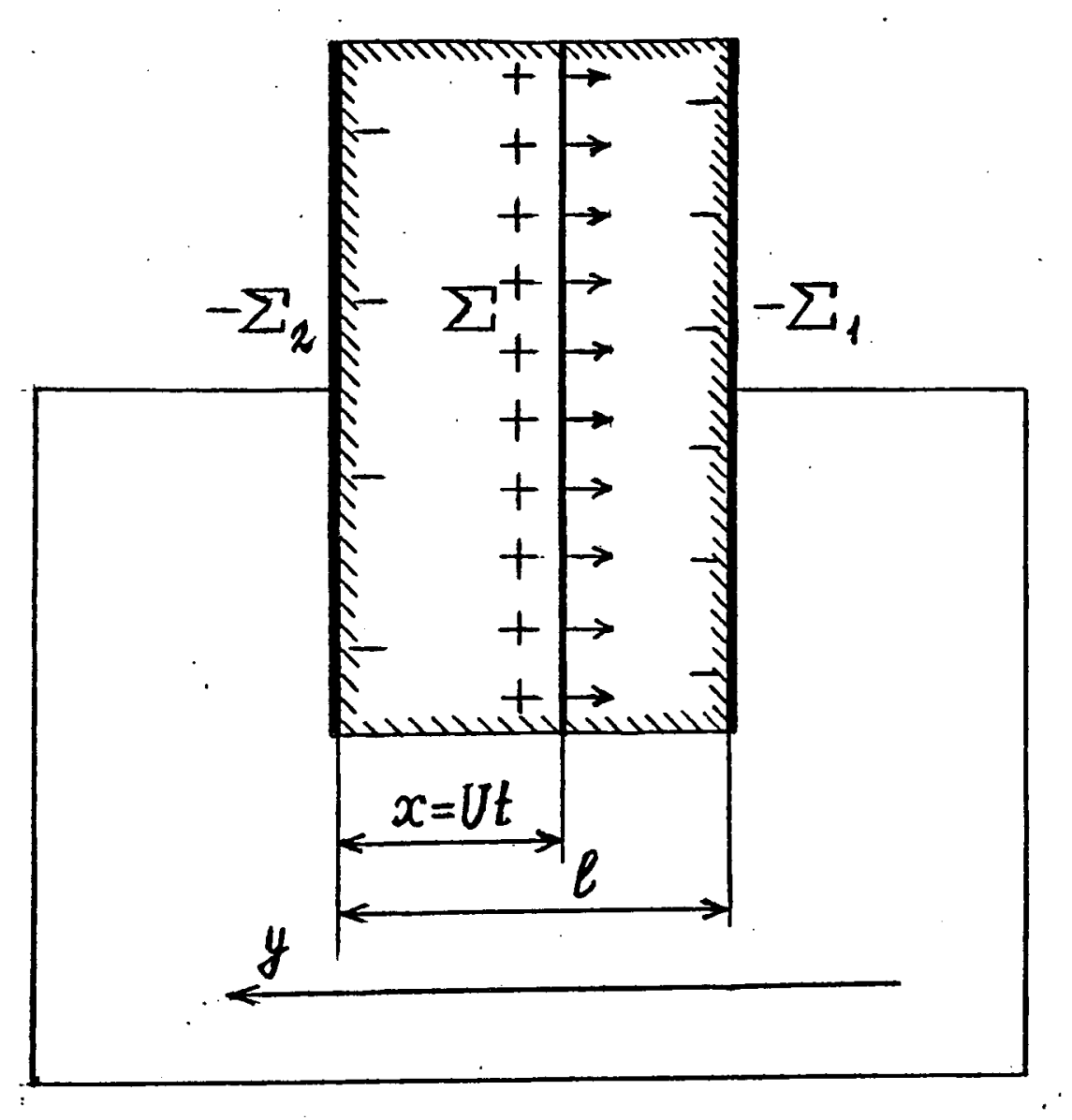

Figure 3.2.4. The short-closed condenser.

$\Sigma_{1}$ is a surface density of the right plate charge.

$\Sigma_{2}$ is a surface density of the left plate charge. 


$$
j=\frac{d \Sigma 1}{d t}=\frac{\sum æ T}{[\not T+t(1-\not)]^{2}},
$$

where $T=l / U$ is time of the wave passing through the initial dieleotric thickness $l$, and $x=\varepsilon_{2} k / \varepsilon_{1}$.

Formula (3.2.11) doesn't take into account that the current in the external circuit wasn't formed by a sudden change in the real experiments. Finite time $\tau * R \cdot C$ is necessary for its formation, where $C$ is the capacity of the extemal circuit elements, and $R$ is the load resistance. In other respects this formula gives a satisfactory description of the first phase of the signal, shown at Figure 3.2.2.

If we substitute the expression (3.1.10) in the formula (3.1.11), we'll receive the follow estimation of the current density amplitude $g_{m}=g(0)$ :

$$
j_{\dot{m}}=\frac{\sum \varepsilon_{1} U}{k \varepsilon_{2} I}=\frac{2 q \delta^{2} \nu, M \gamma_{\mathrm{m}}}{\varepsilon_{2} I\left(1+\gamma_{\mathrm{m}}\right)},
$$

Using the parameters, determined above, and also the next parameters $\gamma_{m}=0.2$ and $l=(0.1-1) \mathrm{cm}$, we can find that $J_{m}=(24-2.4) \cdot 10 \mathrm{~A} / \mathrm{m}^{2}$. That corresponds to the experimentally measured currents by the value order.

Qualitative differences of the current behavior in the condenser circuit from the considered one arise if time of the shock polarization relaxation corresponds to the next condition: $\tau_{r}$ «T. Let's assume, that the matter behind the shock wave front becomes a conductor with the coefficient of the electroconductivity $\sigma_{2}$. Then this condition has the following type: $\tau_{r}=\varepsilon_{2} \varepsilon_{0} / \sigma_{2}^{2}$ « $T$. The shock wave front has the surface charge with density $\Sigma$ (at Figure 3.2.4 the sign "plus" was chosen for a definition). There is a layer of the compensating charge with opposite sign behind this surface charge. Let's estimate the thickness of this layer according to Zeldovich [1967].

Let's connect the coordinate system with the shock wave iront and let $y$-axis will be directed in side opposite to the shock wave motion (see Figure 3.2.4). In this coordinate system the velocity $u$ of the matter, which muns against the shock wave, is determined from the mass conservation law: $u=U / k$. The current density, conditioned by the charged matter flow as 
well as by the conductivity current $\sigma_{2} E_{y}$ is

$$
j_{y}=\sigma_{2} E+U \rho / k
$$

where $\rho$ is the density of the volume charge. Condition of the current stationarity in form of $j_{p}=0$ means that

$$
\rho=-\mathrm{k} \sigma_{2} \mathrm{E} / \mathrm{U}
$$

If we substitute the expression (3.2.13) into the Maxwell's equation, we'll receive the next ratio:

$$
\frac{d E}{d y}=-\frac{\mathrm{kF}_{\mathrm{y}}}{\mathrm{U \tau}}
$$

After the integration of the equation (3.2.14) under the follow condition $E(0)=E_{0}=\Sigma /\left(\varepsilon_{2} \cdot \varepsilon_{0}\right)$ we'll receive the next expressions:

$$
\mathrm{E}=\mathrm{E}_{\mathrm{m}} \exp \left(-\mathrm{y} / \mathrm{y}_{\mathrm{o}}\right), \quad \mathrm{y}_{\mathrm{o}}=\mathrm{U} \tau_{\mathrm{r}} / \mathrm{k},
$$

The influence of the electroconductivity is significant, if the characteristic size of the double electric layer is $y_{0} \ll \tau$, where $l=(0.1-1) \mathrm{cm}$ is the sample thickness. From here we receive the next Iimitation $\sigma_{2} \gg U \cdot \varepsilon_{0} \cdot \varepsilon /(k \cdot l) \approx(20-$ 2) $\cdot 10^{-5} \mathrm{~s} / \mathrm{m}$. Comparison of this value with the experimental value of the electroconductivity of shock-compressed materials (for example, in $\mathrm{NaCl}$ under the pressure $10 \mathrm{GPa} \sigma_{2}=10^{-3}-10^{-5}$ $\mathrm{S} / \mathrm{m}$ (Mineev, [1976]) shows, that the relaxation processes may influence on the signal shape.

For lack of the external load the potentials of the condensed plates might be considered as equal to each other. The potential difference $\Delta \varphi$ and effective capacity $C$ between the shock wave front and the right condenser plate are about

$\Delta \varphi=\int_{0}^{\infty} \mathrm{E}_{\mathrm{p}} \mathrm{dy}=\mathrm{E}_{\mathrm{m}} \mathrm{y}_{0}=\frac{\mathrm{U} \cdot \Sigma}{\mathrm{k} \sigma_{2}}, \quad \mathrm{C}_{\mathrm{e}}=\frac{\varepsilon_{0} \varepsilon_{1} \mathrm{~S}}{1-\mathrm{Ut}}$,

where $S$ is the plate area. The current density in the extemal circuit is

$j=\frac{\Delta \varphi}{S} \cdot \frac{d C_{e}}{d t}=\frac{\varepsilon_{0} \varepsilon_{1} U^{2} \Sigma}{k \sigma_{2}(1-U t)^{2}}$.

As it follow from $(3.2 .17), J \rightarrow \infty$ if $t \rightarrow l / J$. Actually, there is the load resistance in the extemal circuit, and $\sigma_{3}$ has a finite value. Consideration of this circumstance shows that the maximum value of $j$ isn't greater the value of $j$ from 
(3.2.12), which has been found without taking into account the conductivity of the matter in the shook wave.

Formula (3.1.17) as well as (3.1.11) demands the correction for small times because the initial value is $j=0$. If parameter $\tau_{r}$ is less than the circuit relaxation time, than at the beginning time of the current increase is about $\tau_{r}$, and the voltage reaches the value of $\Delta \varphi$, and then the current decreases to the value, determined by. (3.1.17) during $\tau$. If $\tau_{r}>\tau$ the current increases for the time period $\tau$, and then it decreases to the value $(3.1 .17)$ during $\tau_{r}$. In both cases the predicted signal shape corresponds to the experimental dependence, represented at Figure 3.2.2.

3.2.2. Electric field in the growing orack and emission of the particles during the fragmentation of the matter.

Intensive deformation and fragmentation of the solids cause statical and variable electromagnetic fields in the wide frequency band. Electrostatic fields, formed by the charges of the fragmented bodies, were studied in the early works [Frencel, 1948; Leb, 1963]. For example, the charge density on the surface of the splited monocrystals of alkaline-haloid compounds is equal to $(5+7) \cdot 10^{-7} \mathrm{c} / \mathrm{m}^{2}$ [Umusorskaya, 1968; Kornfeld, 1970; 1971; 1975; Nabitovich, 1976; Boev, 1980]. The charges, measured after the destruction of basalt, periodite, fine-grained marble and some other matters have the same values order $\left(10^{-7}+10^{-8} \mathrm{C} / \mathrm{m}^{2}\right)$ [Shevtsov, 1975; Egorov, 1978; Kilkeev, 1979; Parhomenko, 1981; Balbachan, 1983; 1987]. For many minerals and rocks it was founded the appearance of the electric potential, distributed along the sample length, and the arising of the volume charges during one-axis compression of the matter without destruction (for feldspats [Shevtsov, 1975], granite, sandstone, and slates [Egorov, 1978]) and during its extension (for marble and hyposulphite [Kilkeev, 1979]).

On the recently formed surfaces of the destroyed materials the charges are usually distributed as fluctuative mosaic areas with positive or negative charges. Electrometrical measurements by means of the probe showed that in some areas the charge 
density might reach the values of about $10^{-4}+10^{-2} \mathrm{c} / \mathrm{m}^{2}$ [Fincel, $1977 ; 1979]$. Heterogeneous electric structure of the surface is typical for non-destroyed crystals too, with the exception of the especially pure crystals, prepared in vacuum [Vorobiev, 1982]. Linear sizes of negative and positive charged macro-areas are about 50-500 $\mu \mathrm{m}$. But the charge density in these areas is significantly less than on the splited surface.

From the data of Kornfeld [1970, 1971] and Fincel [1977, 1979], after asymmetrical splitting of the sample into two parts, the smallest part more often has a negative potential with respect to the largest part. The explanation of this effect is connected with the transference of the point defects [Kornfeld, 1970] or dislocations [Molotsky, 1976] across the direction of the growth of the magistral crack, destroying the sample. Theoretical analysis [Molotsky, 1976] showed, that the asymmetrical slip of the dislocations in the crack top, where the stresses are especially large, may result in the division of the charges with opposite signs between unequal parts of the destroyed sample. But Boev [1980] carried out the experiments with LiF samples, analogous to the samples, used by Fincel [1979], and results of these experiments showed, that the distribution of the charges according to their signs between two parts of the splitted sample has a causal character. So, the experimental corroboration of this effect remains undecided in spite of the fact, that the densities of the surface charges in both experiments were approximately identical (of about $10^{-6} \mathrm{c} / \mathrm{m}^{2}$ ) [Boev, 1980].

In all experiments the measurement of the sample charge was realized a certain time later the destruction. The charge decreases in time due to the relaxation processes and the effect of the field shielding by the charges of the air ions, adsorbed by the matter surface. So, we can expect that at the dynamic stage of the destruction during the growth of the magistral craok the density of the charges on its sides is much greater than the value, measured experimentally.

Total charge of the destructed body fragments always has a positive sign [Kornfeld, 1970;1971; Kilkeev, 1979]. Most of the investigators assume, that this phenomenon is connected with the process of the short-time emission of the electrons at the 
stage of the matter destruction. In ionic crystals the mechanical emission of the electrons is observed before the appearance of large cracks and splits [Zakrevsky, 1979; Tsa], 1982]. During the quasistatical loading the emission arises when the relative deformation becomes greater its threshold value, that is $0.02+0.1$. The authors [Zakrevsky, 1979] suppose, that this phenomenon isn't connected with single slip, i.e. with the motion and the reproduction of the dislocations, when the intersection of the spliting bands and the appearance of large local deformations take place. They also assume, that the output of the electrons in vacuum is connected with the formation of excitons in the stressed areas. Mechanical luminescence and sharp growth of the conductivity are observed simultaneously with the emission.

Dynamic destruction of the samples is accompanied by the electronic emission too [Deryagin, 1973; 1976]. Krotova [1975] used photomultiplier with the threshold of the registration of $20 \mathrm{KeV}$, that allowed to record, in average, 30 impulses during the destruction of one LiF orystal in vacuum. Average energy of the emitted electrons, registered by this device, was equal to $30 \mathrm{KeV}$. In some cases, this energy achieved $100 \mathrm{KeV}$. During the mica splitting the energy of the mechanical electrons was in range from 10 to $100 \mathrm{KeV}$.

Such large values of the electrons energy can't be the result of the interaction between the point defects of the crystal lattice and the dislocations, as it was suggested by Krotova, [1975]. In some works [Molotsky, 1977; 1983] this effect was explained by the influence of the electric field, formed by chaotic charges on the cracks sides. The electrons, grabed by the surface traps, free themselves due to thermal fluctuations or some other causes, and then they are accelerated by the electric field of the surface charges. Molotsky, [1983] determined the density of the mosaic fluctuating charges as a double-periodic function of $x$ - and $y$-coordinates: $\sigma=\sigma_{m} \cdot \cos (\alpha \cdot x) \cdot \cos (\beta \cdot y)$, where $\alpha$ and $\beta$ are parameters of the charge structure periodicity. Solution of the Laplace's equation for the potential under the corresponding border conditions allowed to find the spatial distribution of the potential and the energy $w$ of the emitted electrons: 


$$
w=\frac{\sigma \cdot e \cdot \cos (\alpha x) \cdot \cos (\beta y)}{\varepsilon_{0}(\varepsilon+1) \sqrt{\alpha^{2}+\beta^{2}}},
$$

where $e$ is en elementary charge. If the spatial structure period is such as $\alpha=\delta=\pi / a$, then the average value of the electrons energy is

$$
\langle w\rangle=\frac{2 \sqrt{2}}{\pi^{3}} \cdot \frac{\sigma_{a} \cdot e \cdot a}{\varepsilon_{0}(\varepsilon+1)} .
$$

This result might be easy explained from the qualitative viewpoint. The strength of the electric field near the surface in vicinity of the centre of the charged cell is determined as $E=\sigma_{\mathrm{m}} / \varepsilon \cdot \varepsilon_{0}$. Because of the sign-variability of the function of the charges distribution the field decreases with a distance from the surface at small distances of about characteristic cell size $a$. Therefore, the emitted electron may have its maximum energy $w \simeq e \cdot E \cdot a \bumpeq e \cdot a \cdot \sigma_{w} / \varepsilon \cdot \varepsilon_{0}$, which coincides with $(3.2 .18)$ and $(3.2 .19)$ by the value order.

It was found that the transversal field in the crack was short-acting. In order to explain how the electrons in this field gather the energy, sufficient for the effect explanation, it is necessary to use the larger local density $\sigma$ of the surface charge. Finkel, [1975] and Molotsky, [1977] found, that during the destruction $\sigma$ must be 2-3 orders greater than the residual charges density."

Surkov, [1986] and Hershenzon, [1986] suggested a different mechanism of the effect of the mechanical emission of the electrons. This mechanism is based on the charges separation between the crack top, its sides, and the sample surface. The electrons gather the energy in far-acting field, directed not across, but along the crack.

Let's consider the effect of the formation of the longitudinal electric field in the crack, which grows in the crystalic dielectric [Surkov, 1986]. Near the crack top the mechanical strengths are larger than the average strengths in the sample by a few orders because of the geometrical factor. So, there is the plastic zone in vicinity of the crack top. We can observe intensive reproduction of the dislocations and point defects in this zone. So, the processes, analogous to the 
processes, which result in the charges separation at the shock wave front, arise in the plastic zone. Iet's use the equation (3.2.2)-(3.2.4) to describe them.

Let's investigate two types of the defects with opposite signs $\pm q$. If $l=1$ let's multiple the equation (3.2.3) by $q_{1}=q$, and when $i=2$ let's multiple this equation by $q_{2}=-q$. If then we sum up these results, we'Il receive the equation of the continuity for the current density $\vec{J}$

$$
\frac{\partial \rho}{\partial t}+\nabla \vec{J}=0, \quad \vec{J}=\rho \vec{\nabla}-q \delta^{2} \cdot \nabla\left(\nu_{1} \mathrm{~N}_{1}-\nu_{2} \mathrm{~N}_{2}\right)+\left(\sigma_{1}+\sigma_{2}\right) E .
$$

We consider the mobility and the electroconductivity of the defects of the first type to be greater than these properties of the defects of the second type, and $v_{1}=$ const. Then, if we transform the equation (3.2.20) with help of (3.2.4) and neglect the current, conditioned by the medium motion, we'll receive the next expression:

$\frac{\partial \rho}{\partial t}+\frac{\rho}{\tau}+\nabla \vec{J}_{d}=0, \quad \vec{J}_{d}=-q \cdot D \cdot \nabla N$,

where $\tau=\varepsilon \cdot \varepsilon_{0} / \sigma_{1}$ is the charge relaxation time. $D=\nu_{1} \cdot \delta^{2}$ is the diffusion coefficient, and $\vec{J}_{\|}$is the density of the diffusion current. In the case of the dislocations $D=c_{d} \cdot \delta$.

Estimations of the terms in the Maxwell's equations show, that the risen problem is quasistatic. So, the boundary conditions on the crack side-air (or vacuum) interface, are the next:

$\frac{\partial \Sigma}{\partial t}=\sigma E_{n}-q D \cdot \frac{\partial N}{\partial n}, \quad \Sigma=-\varepsilon \varepsilon_{0} E_{n}$,

where $\Sigma$ is the surface charge density. Index $n$ designates the direction of the normal to the surface. Besides that, in this expression we took into account the fact, that all surfaces are plate and $E_{n}=0$ because of the symmetry of the task in the air.

Let's substitute the charge density from (3.2.21) in the Poisson's ratio for the potential $\varphi$ of the quasistationary electric field:

$\Delta \varphi=\frac{\exp (-t / \tau)}{\varepsilon \varepsilon_{0}} \int_{0}^{t} \exp \left(t^{\prime} / \tau\right) \cdot \nabla \vec{J}_{d}\left(\vec{r}, t^{\prime}\right) d t^{\prime}$.

where $\Delta$ is the Laplace's operator. 
Let's investigate a thin crack, moving in the plane $\mathrm{z}, \mathrm{x}$ in the sample, which is infinite in z-axis direction (see Figure 3.2.5). We'Il limit ourselves to the case, when the function $N$ might be approximated by means of the expression of the follow type:

$q D N=A(1-\exp (\zeta / \lambda)) \cdot \exp \left(-|y| / \lambda_{\perp}\right) \cdot \eta(-\zeta), \quad \zeta=x-v_{c} t, \quad(3.2 .24)$ where $A=$ const, $\eta$ is the unit step function, and $v_{c}$ is the velocity of the crack growth. In accordance with (3.2.24) the defects distribution is stationary in vicinity of the crack top. Their density decreases at the lengths of about $\lambda$ and $\lambda_{1}$, which are the characteristic scales of the plastic zone in the longitudinal and transversal directions.

The case, when the length of the charge relaxation is $\tau_{r}=v_{c} \cdot \tau \gg \lambda, \lambda_{\perp}$ is the most interesting. The task solution show, than in the limits $l_{r} \gg v_{c} \cdot t \gg \lambda$ the charge per the unit of the crack front length is determined as $Q=2 \cdot A \cdot t \cdot \lambda_{1} / \lambda$. It is possible to show, that the same charge with the opposite sign is located in the double electric layer with thickness of about $\lambda_{\perp}$, formed by the volume charges on the cracks sides. If $t \gg \tau$, these charges are $Q=2 \cdot A \cdot \tau \cdot \lambda_{1} / \lambda$.

Schematic charges distribution for $q>0$ is represented at Figure 3.2.5. In this case the crack top transfers with itself the positive charge $Q$, and its sides are impoverished by the positive charges, because of the defects diffusion.

It is possible to find the distribution of the potential on the crack surface by means of the Green's function from equation (3.2.23), which determines the potential of the charged thread. For example, if $t \gg \tau$ the potential difference between the free surface of the sample $x=0$ and the crack top $\left(v_{c} \cdot t \gg \lambda, \lambda_{\perp}\right)$ is [Surkov, 1986]

$\varphi=-\frac{A t \lambda_{\perp}}{\pi \lambda \varepsilon \varepsilon_{0}} \ln \frac{v t}{\lambda_{\perp}}$.

If $t \gg \tau$, than, at a distance from the crack top, which is greater or equal $l_{r}$ the potential difference is practically independent from $x$ and $t$ (to within $\lambda_{\perp} / l_{r}$ ), and it is determined by $(3.2 .25)$, where $t$ must be changed by the constant $\tau$.

Practically $\varphi$ coincides with the potential of the linear 


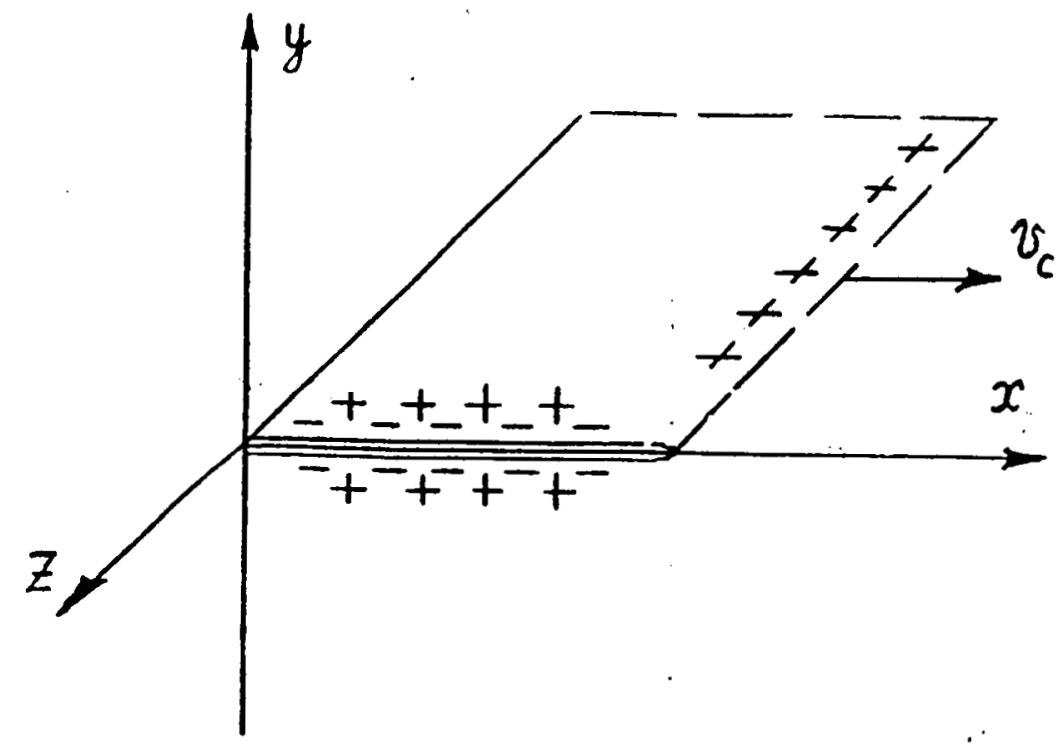

Figure 3.2.5. Schematic charges distribution in the vioinity of the top of a thin crack, moving in the plane $z, x$ in the sample. 
charges $Q$, mentioned above, by the value order, if the distance between them is equal to the crack length $v_{c} \cdot t$ or the relaxation length $l_{\mathrm{r}}$ (if $t \gg \tau$ ).

In the experiments Hershenzon, [1986] received the dependence, similar to linear, between the length of the growing crack and electric signal from the passive antenna, located in the induction (non-wave) zone. This result qualitatively coincides with the dependence (3.2.25).

Inserting into $(3.2 .25)$ the next parameters of the point defects and sample material: $N=10^{23}+10^{24} \mathrm{~m}^{-3}, \nu=5 \cdot 10^{12} \mathrm{~Hz}$, $\delta=3 \cdot 10^{-10} \mathrm{~m}, \quad \lambda_{\perp}=10 \cdot \lambda=1 \mathrm{~mm}, \quad v \cdot t=1 \mathrm{~cm}, \quad \sigma=10^{-5} \mathrm{~s} / \mathrm{m}$, $\varepsilon=5$, gives $\varphi=10^{4}+10^{5} \mathrm{~V}$. The density of the surface charge at the crack front is $\Sigma_{f} \bumpeq Q /(2 \cdot \lambda)=3 \cdot 10^{-3} \mathrm{c} / \mathrm{m}^{2}$, that is 10 time less than it was suggested by Molotsky, [1983]. There is the possibility of the dislocation mechanism of the charges separation. If $q=1.7 \cdot 10^{-11} \mathrm{c} / \mathrm{m}, N_{d}=10^{16} \mathrm{~m}^{-2}$, and $c_{d}=1.5$ $\mathrm{km} / \mathrm{s}$ we'll receive the values of $\varphi$ and $\Sigma_{f}$ of the same order.

So, the estimation of the potential difference in the longitudinal field of the crack is $10+100 \mathrm{kV}$, that allow to receive the qualitative explanation of the appearance of the electrons with energy of about $10+100 \mathrm{KeV}$ in the experiment. The mechanism of the formation of the longitudinal field in the crack, proposed by Surkov, [1986], might be tested experimentally during the measurements of the angular distribution of the emitted electrons.

Transference of the charge, connected with the crack top, was observed by Hershenzon, [1986] during the disturbanoe of IiF crystals. So, the conolusions of the theory, proposed by Surkov, [1986], and Hershenzon, [1986] are confirmed experimentally.

3.2.3 Electromagnetic field of the shock wave during the underground explosion.

In this part we'II consider large-scale displays of the shock polarization effect, which take place during the underground explosion. Strength waves, spreading in soils and rocks, give birth to the complex of low-irequency electromagnetic effects with different physical nature. So, the 
observation and interpretation of experimental data present some difficulties.

One of the causes of the effects variety consists in the fact, that natural matters and rocks, which are the components of the Earth's crust, are very heterogeneous as it concerns to their reological properties as well as the electric parameters. For example, the rocks electroconductivity depends on their moisture and mixtures concentration. so, the electroconductivity can be changed significantly with the depth. Presence of cracks and pores, which open and close themselves in the shock wave, offers facilities to form local electric fields and charges with large value, and accompanied by micro-breakdown of the medium.

In the heterogeneous medium the effect of the shock polarization arises at different structural levels [Surkov, 1990; 1991; 1992]. At first, it arises at the atomic level, inside separate monocrystals and grains, because of the transference of the dislocations and point defects of the atomic lattice. Secondary, it arise at the microscopic level near the grains borders, microcracks and voids. The effect is observed on macroscopic scales too, when the polarization processes become localized in the areas near the tops of large scales and individual blocks of the destroyed rocks. Because of these causes a certain hierarchy of the relaxation times of the shock polarization is possible. The greatest times of the increase and decrease of the polarization might be a few orders greater than the parameters, observed in the experiments with monocrystals.

Polarization in the different structural units of the heterogeneous matter results in the fact, that the shock wave generates its own electromagnetic, usually low-frequency field. In this part we investigate the field, conditioned only by the effect of the shock polarization. Other physical phenomena will be considered below.

The medium polarization $P$ in individual monocrystals is proportional to the amplitude $P$ of the shock pressure. For example, as it follows from the equation (3.2.9) $P=B \cdot P$, where $B=q \cdot \delta^{2} \cdot v_{*} \cdot M /(U \cdot K)$, and $K$ is the modulus of the volume compression of the medium. Vectoral sum of these values in the 
volume unit will determine the volume polarization in the shock wave.

In Iragile mediums the cracks are formed under the influence of the shock loading. The charge, concentrated at the crack top, is equal to $Q \bumpeq 2 \cdot A \cdot \tau \cdot \lambda_{\perp} / \lambda \bumpeq 2 \cdot q \cdot \nu_{*} \delta^{2} \cdot M \cdot \tau \cdot \lambda_{\perp} \cdot P /(\lambda \cdot K)$ per unit of the length of this separate crack front (see above). The charge with the opposite sign is located at the crack sides at a distance not greater than the length of the charges relaxation $l_{r}$. So, each crack becomes an effective electric dipole with moment directed parallel to the crack sides. Because the cracks grow mainly in the direction of the wave spreading, the average vector of the dipole moment must have the same orientation.

Fluctuation charges on the crack sides form cross-oriented dipoles. But the average dipole moment in this case is, probably, about zero, due to the causal character of these dipoles positions.

In the nearest zone of the strong underground explosion the medium breaks to pieces in the destruction wave. The velocity of the destruction wave after its separation from the main shock wave is about of Rayleigh's wave velocity (. $1 \mathrm{~km}$ ). Similar waves were visually observed in the laboratory experiments during the explosions in the semi-transparent materials [Adushkin, 1961, Tsvetkov, 1977]. Under the conditions of the compression the created cracks mainly have a shear character and they grow at angles of $\theta=0.5 \cdot \operatorname{arcctg} k$ with respect to the compression direction [Cherepanov, 1966]. Here $k$ is the friction coefficient in the formula $\tau_{x y}=-Y+k \cdot \sigma_{n}$, which gives the connection between the tangential $\tau_{\dot{x} y}^{n}$, normal $\sigma_{n}$ strengths, and parameter of medium solidity $Y$. Usually, $\theta$ is ${ }^{n}$ about $45^{\circ}$.

Average polarization $|\langle\phi\rangle|$ of the medium behind the destruction wave front has the following order of the value: $|\langle\Phi\rangle|=Q \cdot l_{\mathrm{r}} \cdot N_{c} \cdot \cos (\theta)$, where $N_{c}$ is a number of the cracks per the area unit. Vector $\langle\bar{r}\rangle$ is oriented along the normal to the wave front, and its modulus is proportional to the pressure $P$ or to the normal compression strength. The cracks concentration $N$ depends on the pressure, so the dependence of $|\langle T\rangle|$ from $P$, evidently, deviates from the linear. 
In future, we'll not concretize the mechanism of the shock polarization, assuming the linear ratio between $P$ and $P$ : $\Phi=B \cdot P$, where parameter $B$ might be determined empirically.

Let's investigate the electromagnetic field, created by the shock wave, which was formed during the underground camouflet explosion [Surkov, 1985; 1986]. The shock wave has a shape, which is close to the sphere, and spreads in the soil with low conductivity. We think, that the medium behind the shock wave front is polarized in the radial direction. Furthermore, the medium polarization has a weak symmetry, which might be connected with the large-scale heterogeneity of the medium, uneven development of the crackness, weak asymmetry of the shock wave front, influence of the gravity force and some other causes. The medium polarization might be described by the follow expression:

$D(r, \theta, t)=\Phi_{0}(r)(1+\beta \cos \theta)\left[1-e^{-\left(t-t_{0}\right) / t} s\right] \cdot e^{-\left(t-t_{0}\right) / \tau} \cdot \eta\left(t-t_{0}\right)$,

$(3.2 .26)$

where $\beta$ is a small parameter, which takes into account the asymmetry relatively some direction, an angle $\theta$ is measured from this direction, $t_{0}=(r-R) / U$ is the moment of the shock wave arrival to the given point $(r, \theta), U=$ const is the shock wave velocity, $R$ is the radius, where the charge is formed at the shock wave, $\eta\left(t-t_{0}\right)$ is Heaviside's unit function, and $t_{s}$ and $\tau$ are the characteristic times of the increase and the relaxation of the shock polarization.

Taking into account the fact, that the peak pressure in the spherical shock wave changes with a distance according to the power law, we can write the polarization amplitude as:

$$
P_{0}(r)=B P_{*}(R / r)^{n} \text {. }
$$

where $P_{*}$ is the pressure at the shock wave front when $\tau=R$. The power index $n$ changes from the value of $n \approx 2$ for the plastic wave to $n \approx 1$ for the elastic wave. Usually, $n \approx 1.6$ for soils [Rodionov, 1971]. Time of the polarization increase is determined by the length of the shock wave front, which usually is proportional to the pressure amplitude [Grigoriev, 1979]. So, we can use the dependence $t_{s}=\tau_{f} \cdot(r / R)^{n}$, where $\tau_{f}$ is time scale parameter. Process of the polarization relaxation is 
determined by the internal causes, such as the medium electroconductivity, thermal disorientation of the molecular dipoles and so on, as well as by the extermal factors. The heating degree of the matter in the wave, time of the unloading or the decrease of the mechanical strengths are the most important among this extemal factors. For the explosions in soils, porous and solid cracked rocks, when the thickness of the shock wave front reaches a few meters, the parameters $\tau$ and $\tau_{f}$ might be significantly greater that for the small-scale explosive waves.

Let the dipole moment $\vec{a}$ of the matter, bordered by the shock wave, is directed along some axis of symmetry $\theta=0$. Let's consider the period, when the shock wave didn't come to the medium surface (the camouflet explosion). If we integrate the projection of $\$(3.2 .26),(3.2 .27)$ to the marked direction by the volume $V$, bordered by the radius $R_{f}=R+U \cdot t$, we'll find unknown value $\vec{a}$. The volume charge of the medium is, mainly, near the shock wave front in the layer with thickness of about $U\left(\tau+T_{s}\right)$. The compensating charge with the sign, opposite to the front, might also be near the explosion cavity.

Because the integral gather its value primary at the length of $U \ll R$, the functions $r^{2} \cdot p_{0}(r)$ and $t_{s}(r)$ under the integral might be considered as roughly constant. In result we receive the following expression:

$d(t)=\int_{\mathrm{V}} P_{\mathrm{r}}(r, \theta, t) \cos \theta d \mathrm{~V}=\frac{4 \pi B P_{*} R^{\mathrm{n}}}{3 R_{\mathrm{f}}^{\mathrm{n}-2}}\left[\tau\left[1-e^{-t / \tau}\right]-\tau_{1}\left[1-e^{-t / \tau} \tau_{1}\right]\right]$, $\tau_{1}=\tau \cdot t_{s}\left(R_{f}\right) /\left[\tau+t_{s}\left(R_{f}\right)\right]$.

It should be noted, that the symmetrical part of (3.2.26) hasn't an influence on the dipole moment (3.2.28) and doesn't give some contribution to the external field.

Behavior of the function (3.2.28) is determined by the relation between the time parameters $\tau_{2}, \tau_{f}$, and $\tau_{m}=R / U$ in it. For typical values: $R=10 \mathrm{~m}, U=5 \mathrm{~km} / \mathrm{s}, \tau, \tau_{\mathrm{f}}=1-100 \mathrm{~ms}$ the next conditions: $\tau_{m} \gg \tau, \tau_{m} \gg \tau_{r}$ might be considered as correct. Taking into account these inequalities, we can replace (3.2.28) by the next approximate expression: 
$d(t)=d_{*} \cdot \alpha(t), \quad d_{*}=4 \pi \beta B P_{*} R^{2} U \tau_{*} / 3$,

$\alpha(t)=\exp \left(-t / \tau_{*}\right)-\frac{\tau}{\tau_{*}} \cdot \exp (-t / \tau)+\frac{\tau / \tau_{*}-1}{\left(1+t / \tau_{m}\right)^{m}}$,

where $m=2 \cdot(n-1)$ and $\tau_{*}=\tau_{f} \cdot \tau /\left(\tau_{f}+\tau\right)$.

To calculate the electromagnetic field of the shock wave at distances significantly greater $R_{\mathrm{f}}$, let's replace the area of the shock compression by the effective dipole with moment (3.2.29), dipped into conductive half-space $\mathrm{z}, 0$ (the Earth), which is contiguous with the non-conductive medium $z$. 0 (the atmosphere). The dipole is placed at the explosion depth $l$, and its vector forms an angle $\psi$ with $z$-axis (see Figure 3.2.6).

For underground nuclear explosion the spectrum of the electromagnetic impulse corresponds to the low-frequency area, as it was shown by the experimental data [Zabloski, 1966; Sweeney, 1989]. The measurements were realized on the Earth's surface at distances of 5-20 km from the explosion epicentre. This area corresponds to the nearest (non-wave) zone for the field, when the wave number $k=\omega / c$ ( $c$ is the light velocity in vacuum) and the frequency $\omega$ answer to the next condition: $k \cdot \rho \ll 1$.

The components of the quasistationary field of the variable point dipole, located in the conductive half-space were calculated by Wait, [1953; 1961] in the Fourier representation. The simplest type of the received formula might be found if parameter $æ=\sqrt{\ell \cdot \sigma \cdot \mu_{0} \omega}$ is limited by the next condition: $|æ| \rho \gg 1$. In the polar coordinates $\rho, z, \varphi$ these formulas are:

$$
\begin{array}{ll}
E_{j}=\frac{\omega d(\omega) e_{j}}{2 \pi \sigma \rho^{3}} \cdot \exp \left(-x_{1}\right), \quad H_{j}=\frac{\omega d(\omega) h_{j}}{2 \pi \bar{x} \rho^{3}} \cdot \exp \left(-x_{1}\right), \quad j=\rho, z, \varphi ;(3.2 \cdot 30) \\
e_{z}=-\left(\omega \tau_{2} \cos \psi+\bar{x} \rho \cdot \sin \psi \cdot \cos \varphi\right), \quad e_{\varphi}=-i h_{\rho}=-2 i \cdot \sin \psi \cdot \sin \varphi, \\
e_{\rho}=i\left(\bar{x} \rho \omega \tau_{2} \cdot \cos \psi-\sin \psi \cdot \cos \varphi\right), & h_{\varphi}=3 i \cdot \sin \psi \cdot \sin \varphi /(\bar{x} \rho), \\
h_{\varphi}=-\left(\omega \tau_{2} \bar{x} \rho \cdot \cos \psi-\sin \psi \cdot \cos \varphi\right), & \tau_{2}=\varepsilon_{0} / \sigma,
\end{array}
$$

where $d(\omega)$ is the Fourier transform of the dipole moment. The angle $\varphi$ is measured from the plane, passing though the vector $\vec{d}$ and z-axis (Figure 3.2.6). If $\omega \cdot \rho \approx c$, the dependence from the 


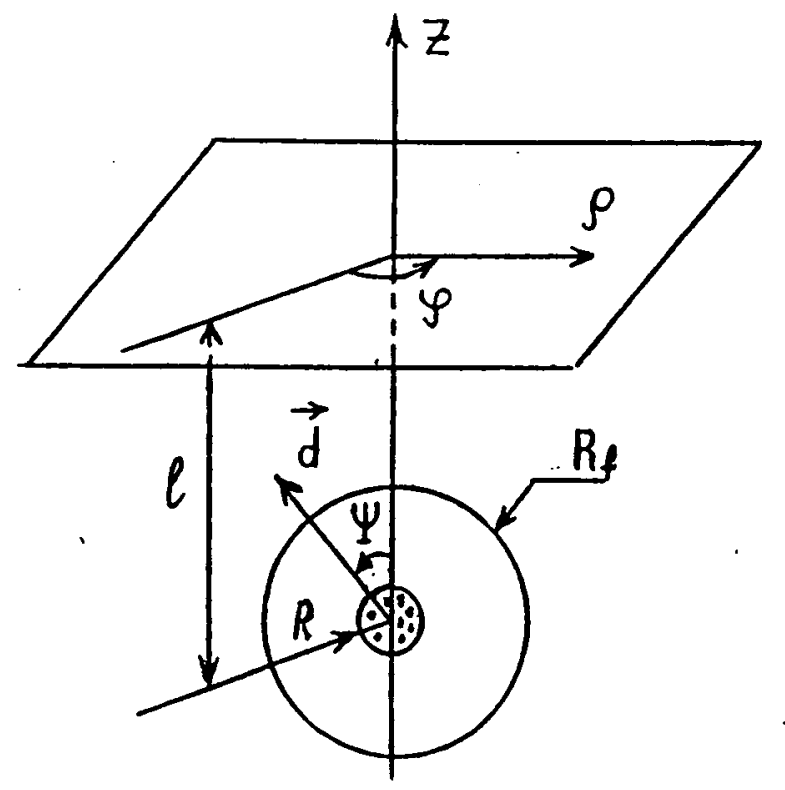

Figure 3.2.6. Disposition of the electric dipole in the ground. 
explosion depth $l$, which was taken into account in the equations (3.2.30), roughly transforms to the accurate dependence.

As it was mentioned above, the truth of the formula (3.2.30) is limited by the follow condition: $\left(\rho^{2} \cdot \sigma \cdot \mu_{0}\right)^{-1} \ll \omega \ll c / \rho$. Using $\rho=7-8 \mathrm{~km}$ and the value of the coefficient of the Earth's electroconductivity of $\sigma=10^{-2}+10^{-3} \mathrm{~S} / \mathrm{m}$, we receive that $(1+16) \mathrm{Hz} \ll \omega \ll(38+43) \mathrm{kHz}$. So, the formula are practically available for whole frequency band of the signals, typical for the electromagnetic field of the underground explosion.

Using the Fourier transform to the equation (3.2.29) by time, we find that

$$
\begin{gathered}
d(\omega)=d_{*} \alpha(\omega), \\
\alpha(\omega)=\frac{\tau_{*}}{1+i \omega \tau_{*}}-\frac{\tau^{2} / \tau_{*}}{1+i \omega \tau}+\left(\frac{\tau}{\tau_{*}}-1\right] \tau_{m} \int_{1}^{\infty} \frac{\exp \left[-i \omega \tau_{m}(x-1)\right]}{x^{m}} d x
\end{gathered}
$$

The ratios $(3.2 .30)$ and $(3.2 .31)$ determine the frequency spectrum of the electromagnetic field of the shock wave. It is obvious from these formulas, that the spectrum features are connected with the polus items, which result in the appearance of the maximums of $\left|E_{j}\right|$ and $\left|H_{j}\right|$ when $\omega * \tau^{-1}$ and $\omega * \tau^{-1}$, i.e. for the frequencies from 10 to $10^{3} \mathrm{~Hz}$. The last item in the equation (3.4.6) has contributed heavily in the area of low Irequencies, when $\omega \leq \tau^{-1} \approx 3 \cdot 10^{2} \mathrm{~Hz}$, because in the opposite case there is a quick-oscillating function under the integral. If $\omega \rightarrow 0$, the fields are absent, so the maximum, determined by the characteristic time $\tau$ of the shock wave might be observed in the low-frequency spectrum part.

In common features the type of this dependence conforms to the typical spectra of the signals, which are observed after large-scale underground explosions. The main power-carrying frequencies are removed to the low-frequency part and are in the range from a few $\mathrm{Hz}$ to a few hundreds of $\mathrm{Hz}$. It should be noted, that there are a few physical mechanisms, which result in these appropriatenesses for the nuclear explosions.

In order to estimate the amplitude and the duration of the electromagnetic signals of the shock wave it is necessary to investigate the spatial-temporal representation of the field. 
For this aim the expression (3.2.30) should be integrated by the frequencies. It should be noted, that at the area of the maximums of the expression under the integral, $\omega \cdot \tau_{2} \ll 1$, so the field will be mainly conditioned by the horizontal component of the dipole.

Inverse Fourrier's transform results in the follow expression:

$$
\begin{aligned}
& E_{\rho}=-\frac{d_{*} \tau \cdot \sin \psi \cdot \cos \varphi}{4 \pi \rho^{3}}\left[\frac{\mu_{0}}{\pi \sigma}\right]^{1 / 2} \cdot \int_{0}^{t} \dot{\alpha}\left(t-t^{\prime}\right) I_{0}\left(t^{\prime}\right) d t^{\prime}, \quad E_{\varphi}=2 E_{\rho} t g \varphi, \\
& E_{z}=-\frac{d_{*} \sin \psi \cdot \cos \varphi}{4 \pi \rho^{2}}\left[\frac{\mu_{0}}{\pi \sigma}\right]^{1 / 2} \cdot \int_{0}^{t} \dot{\alpha}\left(t-t^{\prime}\right) L_{1}\left(t^{\prime}\right) d t^{\prime}, \\
& H_{\rho}=-\frac{d_{*} \sin \psi \cdot \sin \varphi}{2 \pi \rho^{3} \sqrt{\pi \sigma \mu_{0}}} \cdot \int_{0}^{t} \alpha\left(t-t^{\prime}\right) I_{1}\left(t^{\prime}\right) d t^{\prime}, \quad H_{\varphi}=-\frac{c t g \varphi}{2} H_{\rho}, \\
& H_{z}=-\frac{3 d * \tau \cdot \sin \psi \cdot \sin \varphi}{4 \pi \rho^{4} \sqrt{\pi \sigma \mu_{0}}} \cdot \int_{0}^{t} \alpha\left(t-t^{\prime}\right) L_{0}\left(t^{\prime}\right) d t^{\prime} ; \quad \tau_{0}=\frac{1}{4} \mu_{0} \sigma \tau^{2}, \\
& I_{0}(t)=t^{-3 / 2} \cdot \exp \left(\frac{\tau_{0}}{t}\right), \quad L_{1}=t^{-5 / 2} \cdot\left(t-2 \tau_{0}\right) \cdot \exp \left(-\frac{\tau_{0}}{t}\right),
\end{aligned}
$$

where the point means the differentiation by time. The formulas are correct not only for the function (3.4.4) but also for all other functions, which describe the change of the dipole moment in time.

Parameter $\tau_{0}$ determines the characteristic time, when currents of conductivity in the ground diffuse from the depth $\tau$ to the Earth's surface. If $l=300-500 \mathrm{~m}$ and $\sigma=10^{-2}-10^{-3} \mathrm{~s} / \mathrm{m}$ we find that $\tau_{0}=8 \cdot 10^{-4}-3 \cdot 10^{-5} \mathrm{~s}$. This value is much less than other time parameters $\tau, \tau_{*}, \tau_{m}$, that means the weak dependence of the signals from the explosion depth with these frequencies and parameters.

Analogous investigations, made by Surkov, [1985; 1986], showed that this signal must have double-polar type (Figure 3.2.7). Initial peak for the electric field components is achieved when $t \approx \tau_{*}$ or $t \approx \tau$. The maximum amplitudes of the field components are estimated as: 
$E_{\rho_{\text {m }}} \approx \beta B P_{*} U R^{2} /\left(\sigma \rho^{3}\right), E_{z_{\text {m }}} \approx \beta B P_{*} U^{2}\left(\tau-\tau_{*}\right) /\left(\sigma l \rho^{3}\right)$.

Substituting the next parameters: $\beta=0.2, \tau_{\mathrm{f}}=1 \mathrm{~ms}$, $\tau=10 \mathrm{~ms}$, and the parameters, mentioned above in the expression (3.4.8) we receive, that $E_{\rho_{\mathrm{m}}} \approx 1.6 \cdot 10^{11} / \rho^{3} \mu \mathrm{V} / \mathrm{m}$ and $E_{z} \approx 1.6 \cdot 10^{9} / \rho^{2} \mu \mathrm{V} / \mathrm{m}$, where $\rho$ is expressed in meters. Using this estimation we find, that $E \approx 1 \mu \mathrm{V} / \mathrm{m}$ at distances of a few kilometers. This value coincides with the experimental data.

When $t>\tau$ and $t>\tau_{*}$ the signal changes its polarity, and then it dumps. If $t \gg \tau_{m}^{*}$ the asymptotic law of the waves decrease has a type of the power dependence on time:

$$
\begin{aligned}
& E_{\rho}=-\frac{2 m\left(\tau-\tau_{*}\right) E_{0}}{\tau_{m}\left(1+t / \tau_{m}\right)^{m+1}} \cdot\left[1+\frac{\sqrt{\pi}}{2} \operatorname{erfc}(1)+\left[\frac{\tau_{0}}{2}\right]^{1 / 2} \cdot \tau_{m}^{-m}\left(\frac{t}{2}\right)^{m-1 / 2}\right], \\
& E_{z}=E_{0} \frac{\rho}{\tau} \cdot\left[\frac{\left(\tau_{*}-\tau\right) \sqrt{\tau_{0}}}{t^{3 / 2}}+\frac{2 m\left(\tau_{*}-\tau\right)}{\tau_{m}\left(1+t / \tau_{m}\right)^{m+1}}\left\{\frac{\sqrt{\pi}}{2} \operatorname{erfc}(1)-\frac{1}{3}-M_{m+1}\right\}\right] .
\end{aligned}
$$

Here we use the next designation:

$$
E_{0}=\frac{d_{*} \sin \psi \cdot \cos \varphi}{2 \pi^{3 / 2} \sigma \rho^{3} \tau_{*}},
$$

and the functions $M_{\mathrm{m}}$ are determined as:

$$
\begin{array}{ll}
M_{1}=\frac{1}{2}\left[\frac{\tau_{0}}{\tau_{m}+t}\right)^{1 / 2} \cdot \ln \frac{\sqrt{\tau_{m}+t}+\sqrt{t}}{\sqrt{\tau_{m}+t}-\sqrt{t}}, & M_{2}=\frac{\sqrt{\tau_{0} t}}{2 \tau_{m}}+\frac{3}{2} M_{1}, \\
M_{3}=\frac{\sqrt{\tau_{0} t}}{4 \tau_{m}}\left(\frac{9}{2}+\frac{t}{\tau_{m}}\right)+\frac{15}{8} M_{1} . &
\end{array}
$$

Components of the magnetic field in the nearest zone might be sign-variable and one-polar in dependence on the parameters values. The amplitude values of the field component might be achieved if $t \approx \tau_{*} \tau / \tau_{m}$, and they are limited by the follow values:

$$
H_{\rho_{\mathrm{m}}} \approx \beta B P_{*} R^{2} U\left(\tau-\tau_{*}\right) /\left(\tau \mu_{0} \sigma \rho^{3}\right), \quad H_{z_{\mathrm{m}}} \approx \beta B P_{*} U_{*}^{3} \tau_{*}^{2} \tau^{2} /\left(\mu_{0} \sigma \tau_{\mathrm{f}} \rho^{4}\right),
$$

Substitution of the numerical values of the parameters in the expression (3.4.9) results in the next estimations 


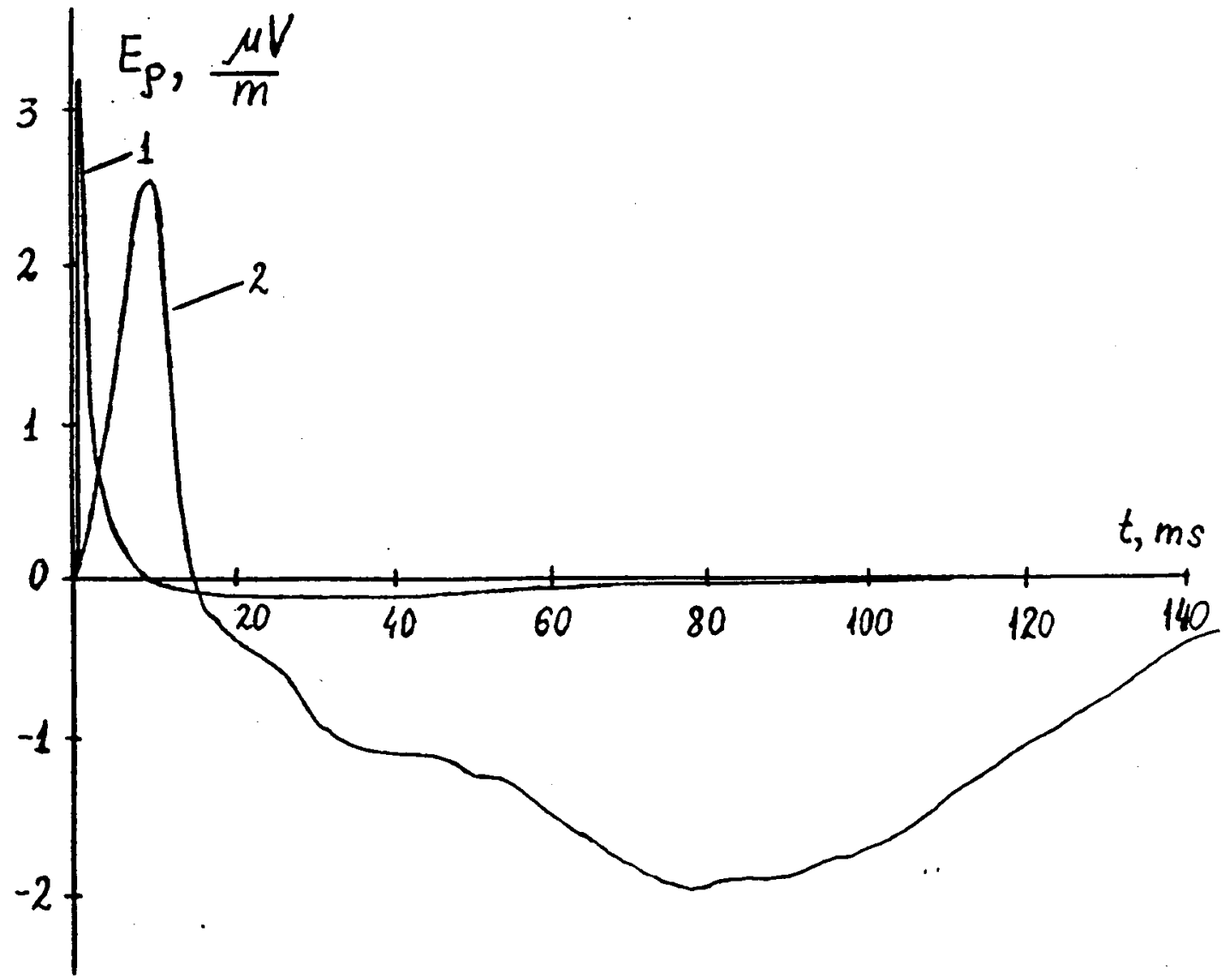

Figure 3.2.7. $E_{\rho}$ of the electric field on the surface at the distance $7.75 \mathrm{~km}$ from the epicenter. $1-E_{\rho}$ calculated on the base of shock polarization effect; 2- experimental curve by Zablocki [1966] for Gnome explosion in halite. 
$H_{\rho} \approx 2.5 \cdot 10^{12} / \rho^{3} \mu \mathrm{A} / \mathrm{m}, \quad H_{z} \approx 3 \cdot 10^{15} / \rho^{4} \mu \mathrm{A} / \mathrm{m}$, where $\rho$ is expressed in meters. If $\rho \approx 10 \mathrm{~km}$ than $H \approx 1 \mu \mathrm{A} / \mathrm{m}$, that corresponds to the values, observed experimentally.

Decrease of the magnetic components of the signals with great times $\left(t \gg \tau_{m}\right.$ ) submits to the power dependencies, analogous to the expression $(3.2 .34)$ :

$$
\begin{aligned}
& H_{\rho}=H_{0} \frac{2 \rho}{3 \tau} \cdot\left[\frac{\tau^{2}-\tau_{*}^{2}}{\sqrt{\tau_{0} t^{3 / 2}}}+\frac{2\left(\tau-\tau_{*}\right)}{\tau_{0}\left(1+t / \tau_{m}\right)^{m}}\left\{\frac{\sqrt{\pi}}{2} \operatorname{erfc}(1)-\frac{1}{3}-M_{0}\right\}\right], \\
& H_{z}=\frac{2 H_{0}\left(\tau-\tau_{*}\right)\left[1+\sqrt{\pi} \cdot \operatorname{erfc}(1) / 2+M_{m}\right]}{\tau_{0}\left(1+t / \tau_{m}\right)^{m}}, H_{0}=\frac{3 d_{*} l^{2} \sin \psi \cdot \sin \varphi}{8 \pi^{3 / 2} \tau_{*} \rho^{4}} \text {. }
\end{aligned}
$$

To illustrate these dependencies the numerical calculations on the base of the formulas (3.2.29) and (3.2.32) were carried out. Figure 3.2 .7 represents the comparison of the theoretical dependence (curve 1) and the experimental signal (curve 2), received for the underground nuclear explosion "Gnom", which was carried out near Carlsbad, New Mexico, in 1961 [Zablocki, 1966]. The explosion with power of $3.1 \mathrm{kT}$ was realized in the rock-salt at depth of $l=361 \mathrm{~m}$. The experimental signal, shown at Figure 3.2 .7 , was received on the surface at a distance of $\rho=7.5 \mathrm{~km}$ to the North from the explosion. The dependence $E_{\rho}(t)$ and $E_{\varphi}(t)$ are roughly proportional to each other in accordance with the formulas (3.2.32), that allow to determine the angle $\varphi$ of the slope of the effective dipole moment vector to the vertical axis on the base of these data.

The curve 1 was plotted with the follow parameters: $B=1.6 \cdot 10^{-5} \mathrm{C} /\left(\mathrm{m}^{2} \cdot \mathrm{GPa}\right) ; \quad n=1.6 ; \quad \beta=0.2 ; \quad P_{*}=100 \mathrm{GPa}$; $R=100 \mathrm{~m} ; \quad U=5 \mathrm{~km} / \mathrm{s} ; \quad \tau=50 \mathrm{~ms} ; \quad \tau_{\mathrm{f}}=1 \mathrm{~ms} ; \sigma^{*}=10^{-3} \mathrm{~s} / \mathrm{m}$; $\tau=361 \mathrm{~m} ; \rho=7.75 \mathrm{~km} ; \psi=\pi / 2 ; \varphi=-0.22$. Coefficient $B$ was taken from the data of the experimental shock compression of NaCl monocrystals. Figure 3.2 .8 represents the components of the magnetic field strength, calculated with the same parameters.

Positions of the initial peaks of the theoretical and experimental dependencies are different (Figure 3.2.7). This circumstance might be connected with the fact, that the 


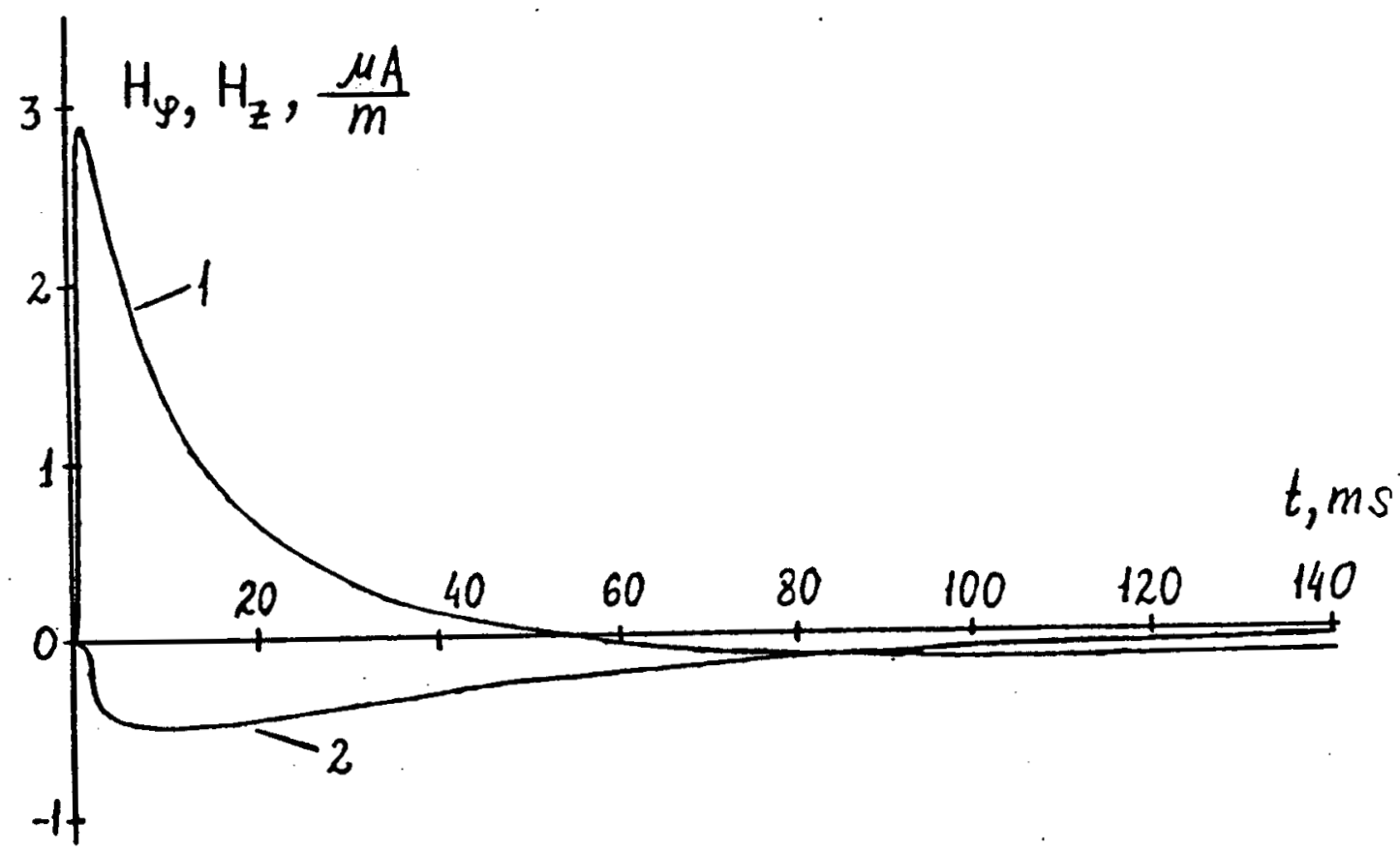

Figure 3.2.8. Magnetic field components on the interface at the distance $7.75 \mathrm{~km}$ from the epicentre that was calculated on the base of the shock polarization effect. $1-H_{z} ; 2-H_{\varphi}$ 
low-frequency equipment with bandpass from 0 to $200 \mathrm{~Hz}$ was used in the experiment [Zablocki, 1966]. It might leads to increased of rise time of the initial spike and to decreased of the magnitude of the initial spike.

Output of the shock wave at the surface 70-100 ms later the explosion is accompanied by splitting phenomena and additional electromagnetic effects. The formulas, adduced above, don't take into account these processes and subsequently, don't describe the negative phase of the signal, shown at Figure 3.2.7 completely. Furthermore, the high-temperature plasma in the explosive chamber, effects of the radioactive excitation of the field and other phenomena, typical for the nuclear explosions, which will be considered below; also have a significant influence.

Presence of the peaks with small duration of about $\tau_{f}$ or $\tau$ in signals for all field components is connected with fast change of the dipole moment of the shock wave at the beginning of the process. When the longitudinal size of the relaxation zone, which is about $U\left(\tau_{f}+\tau\right)$ becomes small in comparison with the distance, passed by the shock wave, the dipole moment changes only because of the increase of the polarized matter volume and of the decrease of the wave amplitude. The decrease of the electromagnetic field at this stage is determined by the time of the shock wave development, which is about $U / R_{f}$.

In the experimental investigations it was found, that the relaxation time of the electormagnetic impulse for underground nuclear explosions is proportional to $w^{1 / 3}$, where $w$ is the explosion power [Zablocki, 1966]. Within the limits of the shock polarization mechanism this fact might be explained by the proportionality of $R_{f}$ to $w^{1 / 3}$.

The weak dependence of the signals amplitude from the explosion depth is connected with the fact, that the characteristic width of the skin-layer for the electromagnetic disturbances, which is about $\left(\mu_{0} \cdot \sigma \cdot w\right)^{-1 / 2} * 3 \mathrm{~km}$, is significantly greater than $l \cong 0.3 \mathrm{~km}$.

At distances of $\rho \approx 1 \mathrm{~km}$ all components of the electric field strength have roughly the same order, but at greater distances $E_{z}$ decreases slower. If $\rho \geq 1 \mathrm{~km}$, the components $H_{\rho}$ and $H_{\varphi}$ are greater than $H_{z}$. The relation between the field 
components mainly depends on the horizontal component of the effective dipole. The direotion of the dipole vector is determined, apparently, by casual ciroumstance, such as the medium heterogeneity, asymmetry of the crackness development and so on. 
3.3. Electromagnetic signals generated by the seismic waves.

In 1887 the magnetometers of 13 observatories registered the signals from Riviera earthquake [Fleman, 1966; Davison, 1905]. Although it was found, that the signals were induced by the motion of the magnetographs (this magnetograph had a suspended permanent magnet) in the seismic wave, the question about the possibility of the appearance of the magnetic disturbances during the motion of the conductive medium in the Earth's magnetic field was risen.

In 1964 the magnetic field disturbances were recorded with help of the inertialess magnetometers after the earthquake in Alaska. The results of the measurements at a distance of 3000 $\mathrm{km}$ from the epicentre of the earthquake with magnitude of 8.5 were represented by Fleman, [1966]. They were the next (in Table 3.3.1. and 3.3.2. this event designated as letter $E)$ : the maximum horizontal component of the mass velocity in the Rayleigh wave was equal to $0.4 \mathrm{~cm} / \mathrm{s}$; the wave period was $20 \mathrm{~s}$; the displacement was $0.1 \mathrm{om}$; the maximum disturbance of the magnetic field $0.2 \gamma$ (the horizontal component of the magnetic field was $78 \mathrm{~m} \gamma$; $1 \gamma=10^{-5}$ gauss) was observed at the moment of the passing of the surface seismic wave maximum through the point of the observation. No disturbances of the magnetic field during the passing of $P$ - and $S$-waves were registered in this experiment.

The disturbances of the magnetic field (from $0.1 \gamma$ to $1 \gamma$ ) were measured during the spreading of the surface waves on the water [Maclure et al., 1964; Kozlov et al., 1971; Gorskaya et al., 1972; Klein et al., 1975; Krotevich et al., 1977].

Theoretical analysis of the magnetic field disturbances is based on the Maxwell equations and on the mechanics of continua. If the magnetic forces are small in comparison with the inertial forces, then the equations of the mechanics and electrodynamics might be separated from each other. It is possible to use the known solution of the seismic and gravitational waves for the calculation of the magnetic disturbances. In the conductive media with low frequencies of 
the oscillations $(\nu \ll 2 \cdot \sigma / \varepsilon$, where $\nu$ is the frequency, $\sigma$ is the conductivity, and $\varepsilon$ is the dielectric permittivity of the medium) we can neglect the displacement currents in comparison with the conductivity currents, and the equation for the magnetic induction $\vec{B}$ is:

$$
\frac{\partial \vec{B}}{\partial t}-D \Delta \vec{B}+\operatorname{rot}[\vec{v} \times \vec{B}]
$$

where $D=c^{2} /(4 \cdot \pi \cdot \mu \cdot \sigma)$ is the coefficient of the magnetic induction diffusion (so called the magnetic viscosity), $c$ is the light velocity in vacuum, $\mu$ is the magnetic permeability, and $\sigma$ is the conductivity of the medium.

Dorman, [1962], described the magnetic field disturbances during the spreading of the gravitational wave (whose amplitude is small in comparison with its length) at surface of the conductive Iiquid in the uniform magnetic field in the case of the small values of the Reynolds magnetic number $\left(R e=\frac{I \cdot U}{D}\right.$, where $L$ is the characteristic size and $U$ is the characteristic velocity of the medium motion) and Hartmann number $\left(H a=\frac{\mu \cdot H \cdot I}{c} \sqrt{\frac{\sigma}{\eta}}\right.$, where $\eta$ is the viscosity and $H$ is the magnetic field strength). In this case it is possible to neglect the derivative by time in the equation (3.3.1). Then, the equation for the disturbance of the magnetic field strength $\pi$ will be the following:

$$
\Delta \vec{r}=-\frac{4 \pi \sigma}{c^{2}} \operatorname{rot}[\vec{v} \times \vec{H}]
$$

The components of the mass velocities for the surface gravitational wave in the liquid spreading in $\mathbf{x}$-direction, are described as:

$$
\begin{aligned}
& v_{x}=a \cdot \omega \cdot e^{k z} \sin (\omega t-k x) \\
& v_{z}=-a \cdot \omega \cdot e^{k z} \cos (\omega t-k x)
\end{aligned}
$$

where $a$ is the amplitude of the displacement in the wave, $\omega=\sqrt{k \cdot g}, g$ is the gravitational acceleration, $k=2 \cdot \pi / \lambda, \lambda$ is the wave length. The liquid fills the lower half-space.

The magnetic field disturbance in each point of the space is the vector rotating in the vertical plane, which passes through the direction of the surface gravitational wave spreading, with the frequency of this wave. The disturbance amplitude decreases exponentially over the liquid surface, but 
below the liquid surface the maximum at the depth of $\lambda /(4 \cdot \pi)$ (where $\lambda$ is the gravitational wave length) precedes to this exponential decreasing.

The value of the modulus of the disturbance on the surface is:

$$
|\vec{n}|=\frac{\sigma \cdot a \sqrt{g \cdot \lambda \cdot \pi\left(H_{x}^{2}+H_{z}^{2}\right)}}{c^{2} \sqrt{2}}
$$

where $\sigma$ is the Iiquid conductivity, $a$ is the amplitude of the displacement in the gravitational wave, $\lambda$ is the wave length, $c$ is the light velocity, $g$ is the gravitational acceleration, and $H_{x}$ and $H_{z}$ are the components of the external magnetic field along the direction of the wave spreading and along the vertical.

The maximum value of the modulus of the disturbance below the liquid surface is two times greater than this value. Disadvantage of the received solution consists in the fact, that the condition of the closing of the magnetic force lines isn't correct in the water (the divergence of the received field isn't equal to 0$)$.

Correct results are represented by Warburton et al., [1964] and Weaver et al., [1965]. Warburton et al., [1964] proposed the method of the calculations of the magnetic field disturbance $\vec{\hbar}$ on the base of Biot-Savart's law, that results in the integration by the volume, where the current with density $\vec{J}$ is running:

$$
\vec{r}=\frac{1}{c} \int_{v}-\frac{\vec{J} \times \vec{r}}{r^{3}} d V
$$

where $\vec{r}$ is the vector from the points of the volume, where the integration is counting, to the point of the calculation of the magnetic field disturbance and

$$
\vec{J}=\frac{\sigma}{C} \vec{v} \times \vec{B}
$$

Weaver et al., [1965] found the common solution for the magnetic field disturbances, induced by the surface gravitational wave, where the complex representation of the mass velocity is:

$$
\vec{v}=-a \cdot \omega(i \cdot \vec{i}+k) \exp (i \omega t-i k \cdot x-k z)
$$

Here $z$ is directed inside the liquid, $\vec{i}$ and $\vec{k}$ are the unit 
vectors along $x-$ and z-axis, $k=2 \cdot \pi / \lambda=\omega^{2} / g$, and $g$ is the gravitational acceleration.

External magnetic field is described as:

$$
H_{0}=H_{0}(\cos \alpha \cos \theta \vec{r}-\cos \alpha \cos \theta \vec{J}+\sin \alpha \vec{k})
$$

where $H_{0}$ is the absolute value of the field, $\alpha$ is the angle of the slope of the field vector to the horizon, and $\theta$ is the angle between $x$-axis and the magnetic meridian.

The solution for $\mathrm{z}<0$ is:

$$
\begin{aligned}
& h_{x}=\frac{i \cdot A}{\beta}\left[1-(1+i \beta)^{1 / 2}\right] e^{k Z} e^{i(\omega t-k x)} \\
& h_{z}=-\frac{A}{\beta}\left[1-(1+i \beta)^{1 / 2}\right] e^{k z} e^{i(\omega t-k x)}
\end{aligned}
$$

and for $z>O$ (the liquid zone):

$$
\begin{aligned}
& n_{\mathrm{x}}=-A\left\{\frac{2(1+i \beta)^{1 / 2}}{1+(1+i \beta)^{1 / 2}} e^{-k z(1+i \beta)^{1 / 2}}-e^{-k z}\right\} e^{i(\omega t-k x)} \\
& n_{\mathrm{z}}=i A\left\{\frac{2}{1+(1+i \beta)^{1 / 2}} e^{-k z(1+i \beta)^{1 / 2}}-e^{-k z}\right\} e^{i(\omega t-k x)}
\end{aligned}
$$

where

and

$$
a=a \cdot k \cdot H_{0}(\sin \alpha+i \cos \alpha \cos \theta)
$$

$$
\beta=\frac{4 \cdot \pi \cdot \sigma \cdot \omega}{c^{2} \cdot k^{2}}=\frac{4 \cdot \pi \cdot \sigma \cdot g^{2}}{c^{2} \cdot \omega^{3}}
$$

For the waves on the water surface with period of $10 \mathrm{~s} \beta$ is about $10^{-3}$. If we use the condition $\beta \ll 1$ in this solution (that is correct when $T \leq 10 \mathrm{~s}$ ), we'II receive, that:

$n_{x}=-\frac{\pi \sigma a g}{c^{2} \omega}\left[H_{o x} \cos (\omega t-k x)+H_{o z} \sin (\omega t-k x)\right]\left\{\begin{array}{l}e^{k z}, \text { if } z<0 \\ e^{-k z}(2 k z-1), \\ \text { if } z>0\end{array}\right.$

$n_{z}=-\frac{\pi \sigma a g}{c^{2} \omega}\left[H_{o z} \cos (\omega t-k x)+H_{o x} \sin (\omega t-k x)\right]\left\{\begin{array}{l}e^{k z}, \text { if } z<0 \\ e^{-k z}(2 k z+1), \\ \text { if } z>0\end{array}\right.$

Solution of the task of the excitation of the quasistatic electromagnetic field by the surface Rayleight wave in the elastic conductor, which fills the half-space and is located in the constant extermal magnetic field, was found by Gulielmi, [1986]. Gulielmi, as well as Dorman, [1962], and Weaver, 
[1965], neglected the displacement currents in the conductive medium and in the air. For the magnetic field disturbance in the air we receive Laplace's equation. The expression for the amplitude of the magnetic field disturbances on the surface of the elastic medium and the estimation of the magnetic effect of the seismic waves were represented in these work too. If $C_{t}=3 \cdot 10^{5} \mathrm{~cm} / \mathrm{s}$,

$\xi * 0.5 \mathrm{~cm}$ $\sigma \cdot 10^{9} \mathrm{~s}^{-1}(\sim 0.1 \mathrm{~s} / \mathrm{m})$, $B \cdot 5 \cdot 10^{-5} \mathrm{~T}$, $\sigma=10^{8}+10^{9} \mathrm{~s}^{-1}(\approx 0.01+0.1 \mathrm{~s} / \mathrm{m})$ for the river water, and $\sigma \sim 2 \cdot 10^{8} \mathrm{~s}^{-1}(\approx 0.025 \mathrm{~s} / \mathrm{m})$ for the soil with mean moisture $)$, the disturbance of the magnetic induction is $b . \frac{4 \pi \sigma \xi C_{t}}{c^{2}} B \sim 10^{-10} \mathrm{~T} \cdot 0.1 \%$. It should be noted that we used International system of unit as well as CGS in this report.

The magnetic field disturbances during the motion of the conductive elastic medium in the extermal magnetic field were also calculated by Gorbacher et al., [1987]. For $R$-wave the medium velocities were defined as:

$$
\begin{aligned}
& U_{\mathrm{x}}=\frac{v_{0}}{q}\left(e^{-k q z_{-}} \frac{2 q S}{1+S^{2}} e^{-k S z}\right) e^{i(k x-\omega t)} \\
& U_{\mathrm{x}}=i v_{0}\left(e^{-k q z_{-}} \frac{2}{1+S^{2}} e^{-k S z}\right) e^{i(k x-\omega t)}
\end{aligned}
$$

where $q=\left[1-\frac{C_{\mathrm{R}}^{2}}{C_{1}^{2}}\right]^{1 / 2}, S=\left[1-\frac{C_{\mathrm{R}}^{2}}{C_{\mathrm{t}}^{2}}\right]^{1 / 2}, k=\omega / C_{\mathrm{R}}, C_{\mathrm{R}}, C_{1}$, $C_{t}$ are the Rayleight, longitudinal and transversal velocities, $v_{0}$ is the amplitude of the component of the velocity $U_{z}$ (the medium occupies the half-space $z>0$ ).

The magnetic field disturbances in the elastic medium $(z>0)$ are:

$$
\begin{aligned}
& h_{\mathrm{x}}=\frac{v_{0} k}{D}\left(a_{1} e^{-p z}+b_{1} e^{-k q z}+b_{2} e^{-k S z}\right) e^{i(k x-\omega t)}, \\
& h_{\mathrm{y}}=\frac{i v_{0} k\left(1-q^{2}\right)}{q D}\left(a_{2} e^{-p z}-b_{3} e^{-k q z}\right) e^{i(k x-\omega t)}, \\
& h_{z}=\frac{v_{0} k}{D}\left(a_{3} e^{-p z}+\frac{i b_{1}}{q} e^{-k q z}+\frac{i b_{2}}{S} e^{-k S z}\right) e^{i(k x-\omega t)},
\end{aligned}
$$


where

$$
\begin{aligned}
& b_{1}=\left(i \cdot q \cdot H_{0 \mathrm{x}}-H_{0 \mathrm{z}}\right)\left(\frac{\omega^{2}}{C_{1}^{2}}-\frac{i \cdot \omega}{D}\right)^{-1}, \\
& b_{2}=\frac{2 S}{1+S^{2}}\left(S \cdot H_{0 z}-i \cdot H_{0 \mathrm{x}}\right)\left(\frac{\omega^{2}}{C_{z}^{2}}-\frac{i \cdot \omega}{D}\right)^{-1}, \\
& b_{3}=H_{\circ y}\left(\frac{\omega^{2}}{C_{1}^{2}}-\frac{i \cdot \omega}{D}\right)^{-1}, \\
& a_{1}=-\frac{p}{k+p}\left[\frac{1+q}{q} b_{1}+\frac{1+S}{S}\right], \\
& a_{2}=b_{3}, \\
& a_{3}=\frac{i \cdot k}{p} a_{1}, \\
& p=\left(k^{2}-i \cdot \omega / D\right)^{1 / 2},
\end{aligned}
$$

and in vacuum $(z<0)$ they are determined as:

$$
\begin{aligned}
& h_{\mathrm{x}}=a_{1} e^{k Z} e^{i(k x-\omega t)} \\
& n_{\mathrm{y}}=0 \\
& n_{\mathrm{z}}=a_{3} e^{k Z} e^{i(k x-\omega t)} \\
& a_{1}=\frac{v_{0} k}{D(k+p)}\left[\frac{(k \cdot q-p)}{q} b_{1}+\frac{(k \cdot S-p)}{S} b_{2}\right] \\
& a_{3}=-i \cdot a_{1}
\end{aligned}
$$

For estimation of the magnetic field disturbance on the medium surface, the next values were used: $\omega=2 \cdot \pi \cdot c^{-1}$, $C_{\mathrm{R}}=3 \mathrm{~km} / \mathrm{s}, \quad H_{0}=40 \mathrm{~A} / \mathrm{m}(0.5$ oersted $), \quad v_{0}=0.1 \mathrm{~m} / \mathrm{s}$, $\sigma=25 \mathrm{~s} / \mathrm{m}$. The conclusion that the magnetic field was "freezed" and too high magnitudes of the magnetic field disturbances were received because of the conductivity too high. If we substitute the experimental values from [Anisimov et al., 1985]: $v_{0}=3.8 \mathrm{~cm} / \mathrm{s}, \quad H_{0}=40 \mathrm{~A} / \mathrm{m}(0.5$ oersted $), \quad \lambda .3 .10^{5} \mathrm{~cm}$, $\sigma \approx 2 \cdot 10^{8} \mathrm{~s}^{-1} \approx 0.025 \mathrm{~S} / \mathrm{m}$ to the formula:

$$
n_{\mathrm{m}}=\frac{v_{0} \cdot H_{0}}{k \cdot D}=\frac{2 \cdot v_{0} \cdot H_{0} \cdot \lambda \cdot \sigma}{c^{2}}
$$

we'll receive that $h_{m} .13 \mathrm{~m} \gamma$ (in air $\mu \approx 1$ ).

Another two sources of the surface disturbances were considered by Gorbacher et al.,[1987]: from the force acting on the medium surface perpendicular to it along $x$-axis, and from the point source of the longitudinal waves, rather distant from the free surface. The solutions were found under the condition 
of the neglect of the derivative by time in the equation (3.1.1). In the case of the point source it was found, that the amplitude of the magnetic disturbance decreases with distance as $r^{-1 / 2}$, as well as the displacements and the velocities in the corresponding Rayleight wave. It is quite natural, because the magnetic field disturbances are proportional to the velocity of the medium motion.

3.3.1. Instrumentation and experimental results.

The magnetic field variations were registered by means of the inductive magnetometers made by OKB of Schmidt Institute of the Phisics of the Earth.

The method of the constant field [Berdichevsky, 1968] was used to measure a specific resistance of the soil. In view of the fact, that the measurements were carried out with distances between the feeding electrodes about 100 meters and because of the assumption about the medium uniformity, which isn't correct wittingly, the results can't be considered as a precise data.

Seismic oscillations were recorded by the standart seismometers SM-3 which made by OKB of Schmidt Institute of the Phisics of the Earth. The typical equivalent output of the seismometer was $17 \mathrm{~V}$ per $\mathrm{m} / \mathrm{s}$. The seismometer had a frequency responce of 0.1 to $40 \mathrm{~Hz}$.

In each experiments, the seismometers locations were determined by the aim to register the separated longitudinal and surface seismic waves ( 3 components of the motion). The magnetometers, which registered three components of the magnetic disturbances (vertical and two horizontal - in direction of the wave spreading and perpendicular to it) were installed at the same points. The magnetic detectors were buried in the trench at the depth from 0.5 to 1 meters and were covered by the soil with ramming, or they were concreted at the rock. The seismometers were installed on the horizontal surface of the soil at a distance of about 10 meters from the magnetic sensors.

During the rotation of the magnetic sensor under the influence of the seismic wave in natural magnetic field of the Earth the following signals arises in it: 


$$
\Delta H_{z}=H_{0} \frac{v_{z}}{C_{r}}
$$

where $H$ is the value of the Earth's magnetic field at this point, $\dot{v}_{z}$ is the mass velocity of the motion in the wave, $C_{r}$ is the phase velocity of the seismic wave. Estimations, made on the base of this formula, at all observation points gives the magnitudes, which are at least ten times less than the measured magnitudes.

The explosions characteristics are represented in Table 3.1. The results of the measurements of the seismic wave parameters on the Earth's surface are represented in Table 3.3.1. The letters designate the wave kind (Plongitudinal and $R$ - surface waves) and the velocity component $(z$ - along the vertical, and $\mathrm{X}$ is represented the component along the horizontal line from explosion epicentre).

It should be taken into account that the seismic wave parameters may change their values due to the heterogeneities and different geology of the media. The data, represented in Table 3.3.1., confirm these conclusions too. There are the azimuthal components of the velocities at some observation points. But these component must be absent if there is the symmetry of the motion relatively vertical axis, passing through the explosion centre.

Figure 3.3.1.1 shows the results of the measurements of maximum mass velooities at different distances from the explosion centre. For the calculations (formula (3.3.3.24) see below) we used one of the empirical dependencies, which is shown by the dashed line at Figure 3.3.1.1.:

$$
v_{\mathrm{m}}=3.36 \cdot 10^{5}\left(\frac{q^{1 / 3}}{R}\right)^{1.6}
$$

Here $v$ is measured in $\mathrm{cm} / \mathrm{s}, q$ measured in $\mathrm{kt}\left(1 \mathrm{kt}=10^{6} \mathrm{~kg}\right)$, and $R$ measured in meters. Slope of the dashed line at the figure corresponds to the attenuation of the mass velocity with distance (the power is $n=1.6$ ). 
Table 3.3 .1$.

Event $\mathrm{C}_{1}, \quad \mathrm{C}_{t}, \quad \lg \left(R / q^{1 / 3}\right)$, wave compo- $v, \mathrm{~cm} / \mathrm{s} T, \mathrm{~s}$ No. $\mathrm{km} / \mathrm{s} \mathrm{km} / \mathrm{s} \mathrm{m} / \mathrm{kg}^{1 / 3}$ kind nent

\begin{tabular}{|c|c|c|c|c|c|c|c|}
\hline 14 & 7.5 & 3.6 & 3.88 & $\begin{array}{l}\mathrm{P} \\
\mathrm{R}\end{array}$ & $\begin{array}{l}z \\
x \\
z \\
x\end{array}$ & $\begin{array}{l}0.0085 \\
0.0036 \\
0.023 \\
0.012\end{array}$ & $\begin{array}{c}0.037 \\
0.33 \\
0.25-0.4 \\
0.25-0.4\end{array}$ \\
\hline 15 & 3.76 & 2.17 & 4.03 & $\mathrm{P}$ & $\begin{array}{l}z \\
x\end{array}$ & $\begin{array}{l}>0.2 \\
>0.2\end{array}$ & 0.25 \\
\hline 6 & 3.0 & & 3.26 & $\begin{array}{l}\mathrm{P} \\
\mathrm{R}\end{array}$ & $\begin{array}{l}z \\
x \\
y \\
z \\
x \\
y\end{array}$ & $\begin{array}{l}3.3 \\
2.0 \\
2.6 \\
3.8 \\
2.4 \\
3.6\end{array}$ & $\begin{array}{l}0.2 \\
0.2 \\
0.2 \\
1.0 \\
1.0 \\
1.0\end{array}$ \\
\hline 18 & & 3.75 & 3.51 & $\begin{array}{l}\mathrm{P} \\
\mathrm{R}\end{array}$ & $\begin{array}{l}z \\
X \\
z \\
x\end{array}$ & $\begin{array}{l}2.7 \\
1.2 \\
4.5 \\
2.9\end{array}$ & $\begin{array}{l}0.44 \\
0.54 \\
0.8 \\
0.88\end{array}$ \\
\hline 17 & 4.3 & 2.79 & 3.94 & $\begin{array}{l}\mathrm{P} \\
\mathrm{R}\end{array}$ & $\begin{array}{l}x \\
x\end{array}$ & $\begin{array}{l}0.0045 \\
0.014\end{array}$ & $\begin{array}{l}0.3 \\
0.25\end{array}$ \\
\hline $\mathrm{E}$ & 7.5 & 2.85 & 4.85 & $\mathrm{R}$ & $x$ & 0.4 & 20 \\
\hline
\end{tabular}


Table 3.3.2.

\begin{tabular}{|c|c|c|c|c|c|c|}
\hline $\begin{array}{l}\text { Event } \\
\text { No. }\end{array}$ & $\stackrel{\rho,}{\mathrm{Ohm} \cdot \mathrm{m}}$ & $\underset{\mathrm{m} / \mathrm{kg}^{1 / 3}}{\lg \left(R / q^{1 / 3}\right)}$ & $\begin{array}{l}\text { wave } \\
\text { kind }\end{array}$ & component & $H, \mathrm{~m} \gamma$ & $T, \mathrm{~s}$ \\
\hline 14 & 1300 & 3.88 & $\begin{array}{l}\mathrm{P} \\
\mathrm{R}\end{array}$ & $\begin{array}{l}\mathrm{y} \\
\mathrm{x}\end{array}$ & $\begin{array}{l}32 \\
10.5\end{array}$ & $\begin{array}{c}1.2 \\
0.35-0.6\end{array}$ \\
\hline 15 & 1300 & 4.03 & $\begin{array}{l}P \\
R\end{array}$ & $\begin{array}{l}x \\
y \\
x \\
y\end{array}$ & $\begin{array}{l}360 \\
160 \\
270 \\
300\end{array}$ & $\begin{array}{l}0.9 \\
0.9\end{array}$ \\
\hline 6 & 4.0 & 3.42 & $\begin{array}{l}P \\
R\end{array}$ & $\begin{array}{l}x \\
y \\
x \\
y\end{array}$ & $\begin{array}{l}200 \\
200 \\
200 \\
200\end{array}$ & $\begin{array}{l}0.25 \\
1.0\end{array}$ \\
\hline 8 & 100 & 4.1 & $\begin{array}{l}P \\
R\end{array}$ & $\begin{array}{l}x \\
y \\
x \\
y\end{array}$ & $\begin{array}{r}250 \\
170 \\
300 \\
50\end{array}$ & $\begin{array}{l}0.4 \\
0.6 \\
1.0 \\
1.0\end{array}$ \\
\hline 9 & 100 & $\begin{array}{l}3.24 \\
3.94\end{array}$ & $\begin{array}{l}P \\
P \\
R\end{array}$ & $\begin{array}{l}x \\
y \\
x \\
y \\
x \\
y\end{array}$ & $\begin{array}{r}>2000 \\
>2000 \\
230 \\
200 \\
250 \\
200\end{array}$ & $\begin{array}{l}0.3 \\
0.3 \\
0.3 \\
0.3 \\
1.0 \\
1.0\end{array}$ \\
\hline 12 & 500 & 4.33 & $\begin{array}{l}P \\
R\end{array}$ & $\begin{array}{l}x \\
y \\
x \\
y\end{array}$ & $\begin{array}{l}100 \\
300 \\
300 \\
100\end{array}$ & $\begin{array}{l}0.5 \\
2.0 \\
1.5 \\
1.5\end{array}$ \\
\hline 18 & 1300 & 3.51 & $\begin{array}{l}\mathrm{P} \\
\mathrm{R}\end{array}$ & $\begin{array}{l}x \\
y \\
x \\
y\end{array}$ & $\begin{array}{r}830 \\
1040 \\
1600 \\
1360\end{array}$ & $\begin{array}{l}0.32 \\
0.92 \\
1.24\end{array}$ \\
\hline 17 & 1300 & $\begin{array}{l}3.94 \\
4.12\end{array}$ & $\begin{array}{l}P \\
R \\
P \\
R\end{array}$ & $\begin{array}{l}x \\
x \\
x \\
y \\
x \\
x\end{array}$ & $\begin{array}{c}20 \\
43 \\
8.3 \\
10 \\
33.3\end{array}$ & $\begin{array}{l}0.26 \\
0.1 \\
0.06 \\
0.22\end{array}$ \\
\hline$E$ & 10000 & 4.85 & $\mathrm{R}$ & $x$ & 78 & \\
\hline
\end{tabular}




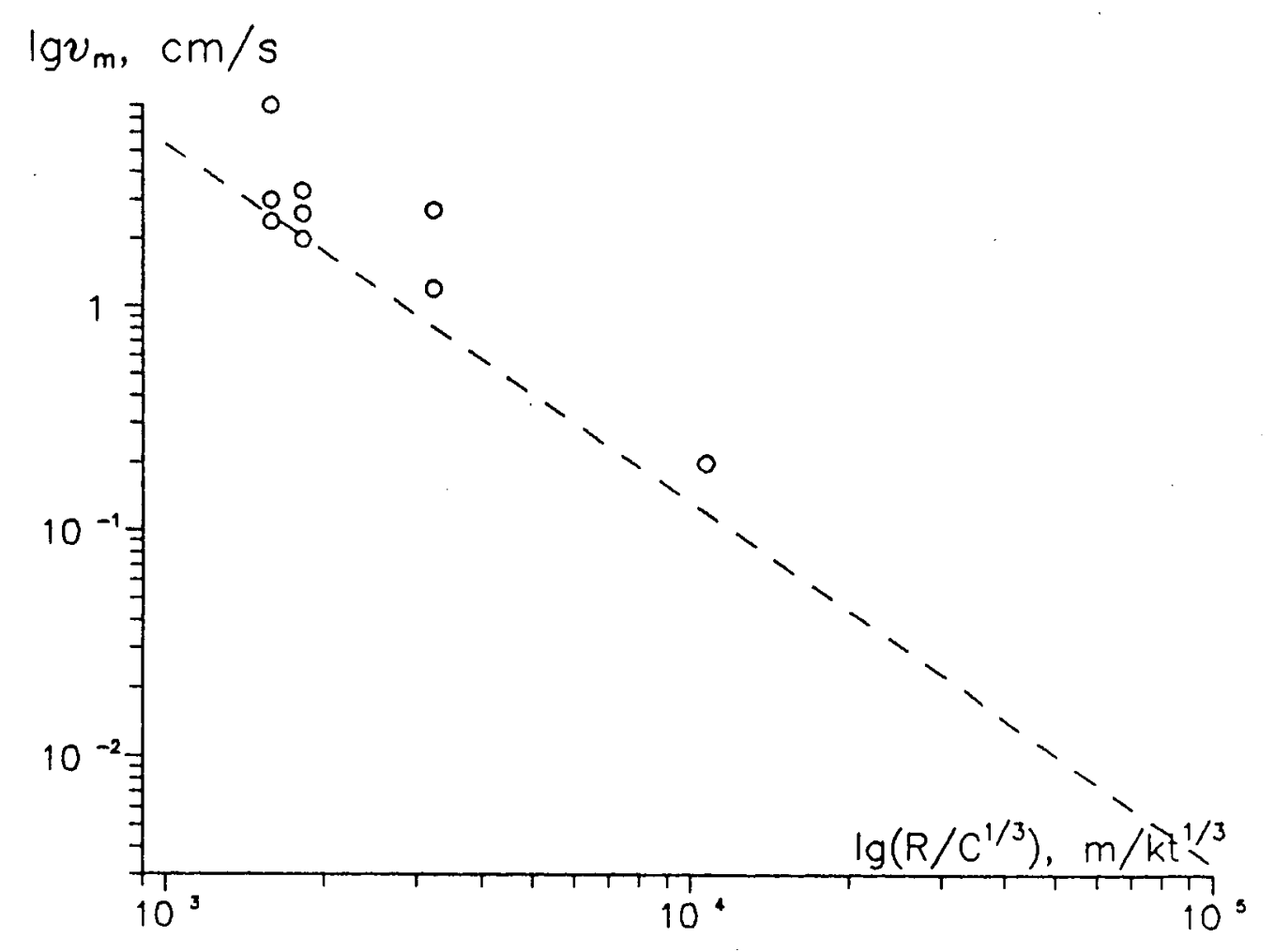

Figure 3.3.1.1. Magnitude of the mass, yelosity
v.s. distance $R / q^{1 / 3}$ 


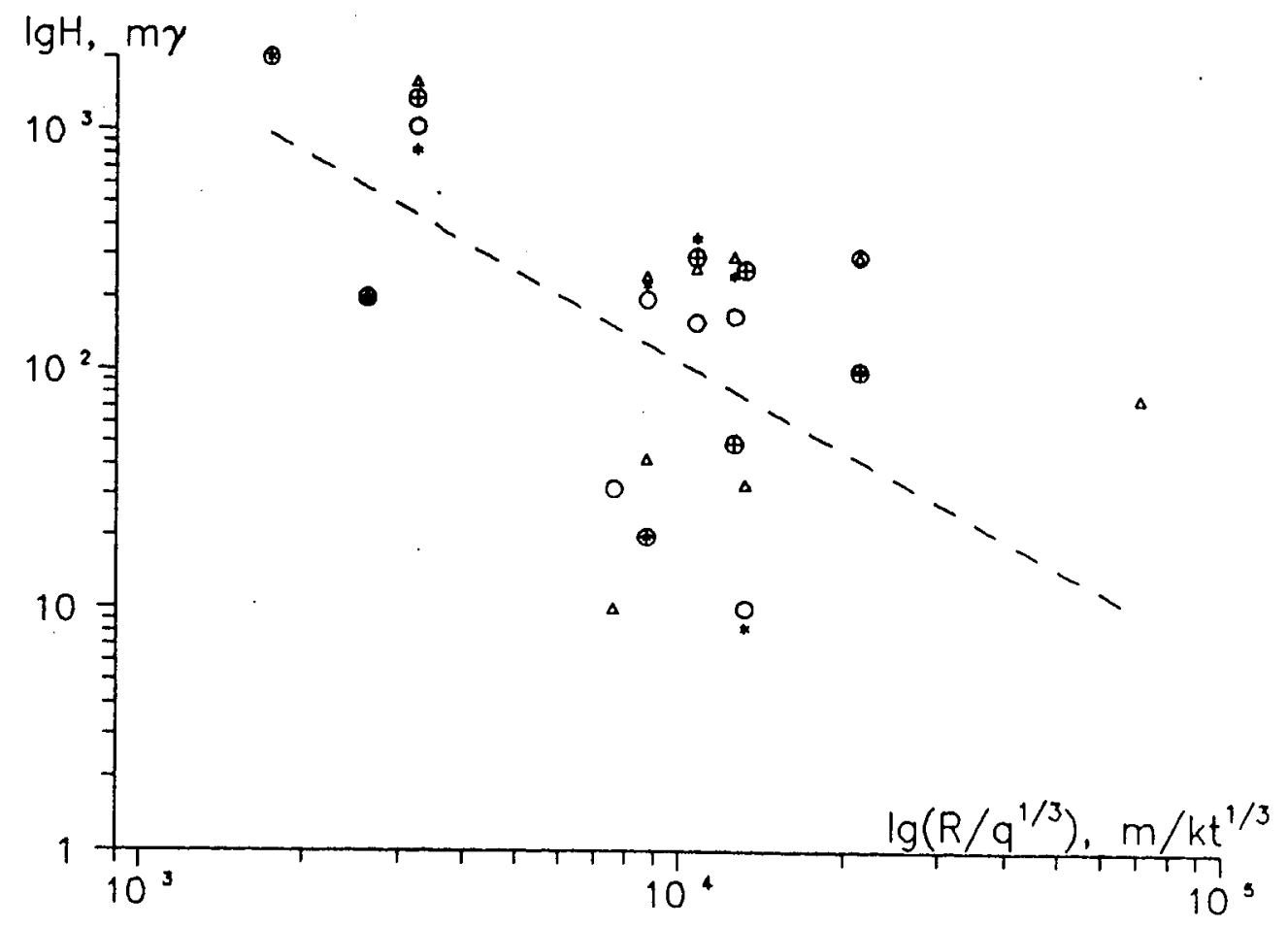

Fig.3.3.1.2. Magnitude of the magnetic field v.s. distance $R / q^{1 / 3}$. 
Table 3.3.2 shows the results of the electromagnetic measurements, where $\rho$ is a specific resistance of the soil.

Figure 3.3.1.2 represents the results of the measurements of maximum amplitudes of the magnetic field disturbance (from Table 3.3.2) in dependence on the distance from the explosion centre.

For the present we don't take into consideration of the change of the conductivity for different soils which may change in time. Although the disturbances are directly proportional to the conductivity, in logarithmic scale they might be not so large at the background of their whole decreasing with the distance.

Also, Figure 3.3.1.2 shows the approximative connection of the magnetic disturbance with the mass velocity of the medium. The slope of the dashed line at the figure corresponds to the attenuation of the magnitude $H$ with distance (the power is $n=1.3$ ). From Tables 3.3.1. and 3.3.2. it is obvious, that the period of the velocities oscillations and the magnetic field disturbances have a large scatter, but in many cases they coincides with each other.

3.3.2. Equations of the motion of the elastic conductive medium in the magnetic field.

The motion of the continua elastic medium in the magnetic field is described by the equation for the displacement of the medium points $\vec{u}$, proposed by Knopoff, [1955], Gulielmi, [1986], Landau, [1965]:

$$
\frac{\partial^{2} \vec{u}}{\partial t^{2}}=C_{t}^{2} \Delta \vec{u}+\left(C_{1}^{2}-C_{t}^{2}\right) \nabla(\nabla \vec{u})+\frac{1}{\rho_{0}} F_{m}
$$

Here $C_{1}$ and $C_{t}$ are the velocities of the elastic longitudinal and transversal waves, which might be represented by means of the compressibility modulus $k$, shear modulus $G$, Lame coefficient $\lambda$, and the medium density $\rho_{0}$ as:

$$
\begin{gathered}
C_{1}=\left(\frac{3 K+4 G}{3 \rho_{0}}\right)^{1 / 2}=\left(\frac{\lambda+2 G}{\rho_{0}}\right)^{1 / 2} \\
C_{t}=\left(\frac{G}{\rho_{0}}\right)^{1 / 2}
\end{gathered}
$$


The force acting on the unit of the medium volume, so called Lorentz force (in CGS system) $F$ is determined as:

$$
F_{1}=\rho_{1} \vec{E}+\frac{1}{C} \vec{J} \times \vec{B}
$$

where $\rho_{1}$ is the space charge density, $\vec{E}$ is the electric field strength, $\vec{B}$ is the induction of the magnetic field, $c$ is the light speed in vacuum, and $\vec{J}$ is the density of the conductivity current, which is determined for many media by the medium conductivity and by the velocity of the medium motion $\vec{v}=\partial \vec{u} / \partial t:$

$$
\vec{\jmath}=\sigma\left(\vec{E}+\frac{\vec{v} \times \underline{B}}{C}\right)+\rho_{1} \cdot \vec{v}
$$

The relation between electromagnetic values is described by the Maxwell equations [Feyman at al., 1966; Tamm, 1956; Landau, 1960]:

$$
\begin{gathered}
\operatorname{rot} \vec{E}=-\frac{1}{C} \frac{\partial \vec{B}}{\partial t} \\
\operatorname{rot} H=\frac{1}{C}\left(4 \pi \vec{\jmath}+\frac{\partial D}{\partial t}\right) \\
\operatorname{div} \vec{D}=4 \cdot \pi \cdot \rho_{1} \\
\operatorname{div} \vec{B}=0 \\
\vec{B}=\mu \cdot \vec{H} \\
\vec{D}=\varepsilon \cdot \vec{E}
\end{gathered}
$$

Some simplifications of this common equations are connected with the task features, which will be considered below. We are interested in the magnetic field disturbance, which arises in the result of the spreading of the surface and volume waves, induced by the explosion in the soil or in the water in the natural Earth's field. The values of the specific resistance (inverse value is conductivity, $\rho=\sigma^{-1}$ ) and the dielectric permittivity of the media necessary for the equations analysis are represented in Tables 3.3 .3 and 3.3.4. The magnetic permeability of the considered media $\mu$ is little different from 1.

The great scatter of the conductivity values attracts our attention in Table 3.3.3. It is evidence of the fact, that the conductivity changes significantly not only for different matters, but also for the same matter.

Iet's begin the analysis from the estimation of time of the electric charge spreading in the soil. On the base of equation (3.3.2.5) in the conductive medium we receive, that 
Table 3.3.3 Specific conductivity of the different mediums, Ohm.m

\begin{tabular}{|c|c|c|c|c|c|}
\hline \multirow{2}{*}{ Medium } & \multicolumn{5}{|c|}{ Author } \\
\hline & Zaborovsky & Enohovich & Abert & Cadastre & Kraev \\
\hline $\begin{array}{l}\text { Wood (dry) } \\
\left.\text { Ice (-12 }{ }^{\circ} \mathrm{C}\right) \\
\text { Sand } \\
\text { Granite } \\
\text { (Krivbass) } \\
\text { Iimestone } \\
\text { Clay (dry) } \\
\text { Water } \\
\text { (distilled) } \\
\text { Soil } \\
\text { River } \\
\text { water } \\
\text { Shale } \\
\text { Schist } \\
\text { Soil (mid. } \\
\text { moisture) } \\
\text { Blood } \\
\text { Sea water } \\
\text { Graphite } \\
\text { Iron } \\
\text { Aluminium }\end{array}$ & $\begin{array}{l}10^{5} \\
10^{4}\end{array}$ & $\begin{array}{l}10^{3}-10^{4} \\
10-100\end{array}$ & $\begin{array}{c}(1 \div 4) \\
10^{4} \\
10^{2}-10^{4} \\
10-100 \\
\\
0.3 \\
0.98 \cdot 10^{-7} \\
2.7 \cdot 10^{-8}\end{array}$ & $\begin{array}{c}4 \cdot 7 \cdot 10^{6} \\
(1.7 \div 3) \\
10^{4}\end{array}$ & $\begin{array}{c}10^{3}-10^{6} \\
10^{2}-10^{3} \\
10^{3} \\
10-10^{4} \\
10-10^{3}\end{array}$ \\
\hline
\end{tabular}


Table 3.3.4. Dielectric permittivity.

\begin{tabular}{|l|c|c|c|}
\hline \multirow{2}{*}{ Medium } & \multicolumn{3}{|c|}{ Author } \\
\cline { 2 - 4 } & Zaborovsky & Kraev & Alpert \\
\hline Sea water & 81 & 80 & 80 \\
River water & & 80 & \\
Distilled & & 80 & \\
water & & & \\
Clay & & \\
(moisture) & 20.0 & & $10-20$ \\
Soil (midd. & 10.0 & 15 & \\
moisture) & 8.5 & $7-12$ & $2+5$ \\
Limestone & 3.5 & & \\
Granite & 2.5 & & \\
Clay (dry) & 2 & & \\
Sand (dry) & & & \\
Soil (dry) & & & \\
\hline
\end{tabular}

$\vec{J}=\sigma \cdot \vec{E}$. After the substituting of this equation and equation (3.3.2.11) to the formula $(3.3 .2 .7)$ we receive, that:

$$
c \cdot \operatorname{rot} \vec{H}=\frac{4 \pi \sigma}{\varepsilon} \vec{D}+\frac{\partial D}{\partial t}
$$

We know, that div rot $H=0$, so on the base of the equation $(3.3 .2 .8)$ we can write:

$$
\frac{\partial t}{\partial t}=-\frac{4 \pi \sigma}{\varepsilon} \rho_{1}
$$

From which:

$$
\rho_{1}=\rho_{10} \cdot e^{-\frac{4 \pi \sigma}{\varepsilon} t} .
$$

Using the data from Tables 3.3 .3 and 3.3 .4 we find, that even for the dry sand, which is the medium with low conductivity $\left(\sigma \approx 10^{5} \mathrm{~s}^{-1}, \varepsilon=2.5\right)$, it is necessary only $2 \cdot 10^{-6} \mathrm{~s}$ to decrease the charge in $e$ times, whereas for the moist soil $\left(\sigma \approx 2.2 \cdot 10^{8} \mathrm{~s}^{-1}, \varepsilon=10\right)$ this time is $3 \cdot 10^{-9} \mathrm{~s}$. This estimation shows, that the free charges disappear in the soil quickly. It is the base to neglect all terms with $\rho_{1}$ in equation $(3.3 .2 .4),(3.3 .2 .5)$, and $(3.3 .2 .8)$.

In the conductive media it is possible to neglect the displacement currents in comparison with the conductivity current if the next conditions are true:

$$
\frac{\partial D}{\partial D^{-}}=\varepsilon \frac{\partial E}{\partial t} \ll 4 \cdot \pi \cdot \sigma \cdot E ; \quad \omega \ll \frac{\sigma}{\varepsilon} ; \quad f=\frac{\omega}{2 \pi} \ll \frac{\sigma}{2 \pi \varepsilon}
$$


It follows from Tables 3.3 .3 and 3.3.4, that for the soil with mean moisture: $f \ll 3.5 \cdot 10^{6} \mathrm{~Hz}$; For the sea water: $f \ll 8.8 \cdot 10^{6} \mathrm{~Hz}$; and for the dry sand: $f \ll 5.7 \cdot 10^{3} \mathrm{~Hz}$. The frequencies, corresponding to the seismic waves of order $1+10 \mathrm{~Hz}$, answer this conditions even in such badly conductive medium as the dry sand. In this case we receive the next equation instead of the formula (3.3.2.7)

$$
\operatorname{rot} \vec{H}=\frac{4 \cdot \pi}{c} \vec{J}
$$

Let's show, that the influence of the electromagnetic forces in the equation of motion is small in comparison with the forces of elasticity, if the motion takes place in the weak magnetic field. For lack of the free charges and the displacement current from equations (3.3.2.4), (3.3.2.12), and (3.3.2.10) we can write, that:

$$
F_{\text {m }}=\frac{1}{C} \vec{J} \times \vec{B}
$$

or

$$
F_{\text {m }}=\frac{1}{4 \pi \mu} \operatorname{rot} \vec{B} \times \vec{B}=-\frac{1}{4 \pi \mu} \vec{B} \times \operatorname{rot} \vec{B}
$$

For the most of the media (with the exception of the ferromagnetic) $\mu$ is about 1 .

If the wire with the cross-section area of $\pi \cdot r_{0}^{2}$ made from the material with conductivity $\sigma$ moves perpendicular to the permanent magnetic field $H_{0}$ with the velocity $v$, and the corresponding current runs through it (close circuit), then we receive the follow expression for the value of the magnetic field $H$, which arise near this wire:

$$
\frac{H}{H_{0}}=\frac{2 \cdot \pi \cdot r_{0} \cdot \sigma \cdot v}{c^{2}}
$$

The magnetic field disturbances induced by the motion are small on condition that velocities of the motion of the conductive medium (soil in this case) in the permanent magnetic field with the induction $\vec{B}_{0}$ are small. So, it is convenient to use the next designation:

$$
\vec{B}=\overrightarrow{B_{0}}+\vec{b}
$$

where $\vec{b}$ is a small parameter, $|\vec{b}| \ll\left|\vec{B}_{0}\right|$.

In this case $\operatorname{rot} \vec{B}=\operatorname{rot} \vec{b}$, and according to the mules of the vectorial product. [Dorman, 1962]:

$$
\vec{B} \times \operatorname{rot} \vec{B}=\nabla(\vec{B} \cdot \vec{D})-(\vec{B} \nabla) \vec{b}
$$


If we neglect the second power in comparison with the first power, will receive:

$$
\vec{B} \times \operatorname{rot} \vec{B} \bumpeq \nabla\left(\vec{B}_{0} \cdot \vec{b}\right)-\left(\vec{B}_{0} \nabla\right) \vec{b}
$$

Now, let's compare the elastic and electromagnetic terms in equation (3.3.2.1). We'Il consider the ratios $u / l$ and $b / B_{0}$ as a small quantity of the first order (a negligibly small). $l$ is a distance, where the motion characteristics changes significantly, here. In our case $l$ is about of the half of the seismic wave length (the wave length is $\lambda=C_{1} \cdot T$, so $\tau$. $1000 \mathrm{~m})$. The order of the eiastic term is $\frac{C_{t}^{2}}{l} \cdot \frac{u}{l}$, the order of the electromagnetic term is $\frac{B_{0}^{2}}{l} \cdot \frac{b}{B_{0}}$, and their ratio is $\frac{B_{0}^{2}}{4 \pi \mu \rho_{0} C_{t}^{2}}=\left(\frac{C_{A 1}}{C_{t}}\right)$, where $C_{A 1}$ is the velocity of the Alfven waves. In our case: $B_{0}=0.5$ gauss, $\mu=1, \rho_{0}=2 \mathrm{~g} / \mathrm{cm}^{3}$, $C_{t}=3 \cdot 10^{5} \mathrm{~cm} / \mathrm{s}$, and this ratio is equal to $10^{-13}$. So, we an neglect the influence of the magnetic forces on the elastic medium motion. Therefore, it is possible to solve the task of the medium motion separately, and then to find the electromagnetic field disturbances, induced by the motion of the conductive medium in the magnetic field, with help of the Maxwell equations. From equations (3.3.2.6) and (3.3.2.5) with regard for the fact, that $\rho_{1}=0$ we receive:

$$
\frac{\partial \vec{B}}{\partial t}=c \cdot \operatorname{rot}\left(\frac{\vec{v} \times \vec{B}}{c}-\frac{\vec{J}}{\sigma}\right)
$$

Using the formulas $(3.4 .12)$ and $(3.4 .10)$ when $\mu=1$ we have that:

$$
\frac{\partial \vec{B}}{\partial t}=\operatorname{rot}[\vec{v} \times \vec{B}]-\frac{c^{2}}{4 \pi \sigma} \operatorname{rot}(\operatorname{rot} \vec{B})
$$

or, because $\operatorname{rot}(\operatorname{rot} \vec{B})=\operatorname{grad} d i v \vec{B}-\nabla^{2} \vec{B}$,

$$
\frac{\partial \vec{B}}{\partial t}=\operatorname{rot}[\vec{v} \times \vec{B}]+\frac{c^{2}}{4 \pi \sigma} \nabla^{2} \vec{B}
$$

For the established (stationary in time) fields in the stationary medium $(v=0)$ these equations can be rewritten simply as the Iaplace's equation

$$
\nabla^{2} \vec{B}=0
$$

In this case the conductive medium hasn't any differences from the non-conductive medium, and the fields are entirely 
determined by the currents according to the Biot-Savart's law

$$
d \vec{H}=\frac{I[d \underline{a} \times \vec{r}]}{c \cdot r^{3}}
$$

where $I$ is the current, and $d l$ is the element of the current line, for which the field is calculating.

Propagation of the field disturbances in the non-conductive medium is described by the follow wave equation:

$$
\frac{\partial^{2} \vec{B}}{\partial t^{2}}=\frac{C^{2}}{\varepsilon \mu} \nabla^{2} \vec{B}
$$

In the conductive stationary medium the transmission of the magnetic field disturbances is determined by the equation of diffusion or heat conductivity, which follows from equation (3.3.2.17) when $v=0$ (the diffusive character of the disturbances spreading is connected with the neglect of the displacement currents in comparison of the conductivity currents):

$$
\frac{\partial \vec{B}}{\partial t}=\frac{c^{2}}{4 \pi \sigma} \nabla^{2} \vec{B}=D \nabla^{2} \vec{B}
$$

Here the value of $D=\frac{c^{2}}{4 \pi \sigma}$, corresponding to the coefficient of diffusion, defines the character of the field change in time and in space. So, time of attenuation of the changing field frequency (in our case it is designated as $b^{*}$ ) is $\tau=\frac{4 \pi \sigma l^{2}}{c^{2}}$, where $l$ is the characteristic size of the area, where the field change is investigated. Time $\tau$ might be considered as the characteristic of the spreading of the field disturbances too, i.e. as time, when the disturbance passes the distance $l=(D \cdot \tau)^{1 / 2}$. In our case $D=3.2 \cdot 10^{11} \mathrm{~cm}^{2} / \mathrm{s}$ for the soil with mean moisture and $D=8 \cdot 10^{14} \mathrm{~cm}^{2} / \mathrm{s}$ for the dry sand. Hence it follows, that during time of about the wave period ( $1 \mathrm{~s})$ the magnetic field disturbance in the moist soil spreads at a distance of about $10 \mathrm{~km}$, and time, when the disturbance passes through the wave area is ${ }^{-1} 10^{-2} \mathrm{~s}$. The depth of penetration $\delta$ of the periodical disturbances into the conductive medium is connected with their frequency as $\delta \cong(D / \nu)^{1 / 2}$. For the frequencies from 1 to $10 \mathrm{~Hz}$ we receive, that $\delta$, $(2+6) \cdot 10^{5} \mathrm{~cm}$ for the soil with mean moisture and $\delta \cdot(0.9+3) \cdot 10^{7} \mathrm{~cm}$ for the dry sand.

So, the magnetic field disturbance penetrates through the 
soil or the dry sand well enough. Using the formula of the vectorial analysis and equation (3.3.2.9) we can transform equation (3.3.2.17) to the following:

$$
\frac{\partial \vec{B}}{\partial t}=\frac{c^{2}}{4 \pi \sigma} \nabla^{2} \vec{B}+(\vec{B} \nabla) \vec{v}-(\vec{v} \nabla) \vec{B}-\vec{B}(\nabla \vec{v})
$$

For the case of small field disturbances (3.4.15) we have:

$$
\frac{\partial \vec{b}}{\partial t}=\frac{c^{2}}{4 \pi \sigma} \nabla^{2} \vec{b}+\left(\vec{B}_{0} \nabla\right) \vec{v}-(\vec{v} \nabla) \vec{b}-\vec{B}_{0}(\nabla \vec{v}) \quad(3.3 .2 .23)
$$

In order to estimate the contribution of the terms in our task, let's transform equation (3.3.2.23) to the dimensionless type. Let's use the next values as the characteristic parameters: $B_{0}$ is the value of the natural magnetic field $\left(b \ll B_{0}\right), C_{t}$ is the shear wave velocity, which in the soil is practically equal to the velocity of the longitudinal and surface waves $\left(v \ll C_{t}\right)$, and $l_{0}$ is the characteristic distance, which might correspond to the area of motion or considerable change of the motion velocity, for example. We'll use here the half of the seismic wave length for the estimations. For the dimensionless values we receive, that $\vec{b}_{\delta}=\frac{\vec{b}}{B_{0}} ; \quad \vec{v}_{\delta}=\frac{\vec{v}}{C_{t}}$, $\nabla_{\delta}=l_{0} \nabla$, and $t_{\delta}=\frac{t \cdot C_{t}}{l_{0}}$. From here:

$$
\frac{\partial b_{\delta}}{\partial t_{\delta}}=\frac{c^{2}}{4 \pi \sigma l_{0} C_{t}} \nabla_{\delta}^{2} \vec{b}_{\delta}+\left(\vec{n}_{B} \nabla\right) \vec{v}_{\delta}-\left(\vec{v}_{\delta} \nabla_{\delta}\right) \vec{b}_{\delta}-\vec{n}_{B}\left(\nabla_{\delta} \vec{v}_{\delta}\right)
$$

where $\vec{n}_{B_{0}}$ is the unit vector in the direction of $\vec{B}$. Inverse value of the first term coefficient in the right part of the equation is known as the Reynolds magnetic number $R_{\mathrm{m}}$ :

$$
R_{\text {m }}=\frac{4 \cdot \pi \cdot \mu \cdot \sigma \cdot \tau_{0} \cdot C_{t}}{c^{2}}
$$

In our case $\mu=1, \sigma * 10^{8} \mathrm{~s}^{-1}(\approx 0.01 \mathrm{~s} / \mathrm{m})$, $C_{t} * 10^{5} \mathrm{~cm} / \mathrm{s}, l_{0} * 5 \cdot 10^{4} \mathrm{~cm}$, and $R_{\mathrm{m}} * 7 \cdot 10^{-3}$. It means, that the magnetic field disturbance $\vec{b}$ approximately answers the Laplace's equation and might be calculated according to the Biot-Savart's law. Under the quasistatic approximation, the contribution of $E$ to the current is small, and the current density $\vec{J}$, in general, is determined by the motion of the medium in the natural magnetic field:

$$
\vec{J}=\frac{\sigma}{c} \vec{v} \times \vec{B}_{0}
$$


For $\vec{b}$ we have the Biot-Savart's formula:

$$
\vec{b}=\frac{1}{c} \int_{v} \frac{\vec{J} \times \vec{r}}{r^{3}} d V
$$

Here we carry out the integration by the volume, where the currents are running, and $\vec{r}$ is the vector from the volume $d V$ to the point of $\vec{b}$ calculations.

3.3.3 Approximate calculations of the seismoinductive effect of the underground explosions.

Electromagnetic disturbances depend on the mass velocity of the medium motion and the medium conductivity.

For a long time the mechanical processes associated with the underground explosion, was an object of a great deal of research. But up to now the satisfactory results were found only for the uniform media (usually in the laboratory experiments) and for the contained explosions. The presence of the free surface, frequently with complex relief, a great number of the heterogeneities, and the differences between the real media result in the large scattering of the measurements results and relatively low accuracy of the motion description, especially in the zone closest to the epicenter.

One of the convenient parameter for the description of the motion in the wave is the maximum mass velocity. The investigations showed, that the geometrical similarity (the proportionality to $(q)^{1 / 3}$, where $q$ is the explosion power) and the power dependence, weakly changing for the different media, are correct for the change of the maximum mass velocity with distance from the explosion. The motion in the seismic waves is the oscillating process, where separated wave groups (such as longitudinal, transversal, surface, and various reflected waves) might be marked out. Although they usually say about the oscillations periods, really no stable harmonic oscillations are observed, but a few oscillations (with different amplitudes, as a rule) may keep time between zero values of the amplitudes.

For the evaluations we'll use the results, represented in Table 3.3.1. and at Figure 3.3.1.1. It should be noted, that available records of the real seismic motion are highly 
different from the harmonic Rayleight wave or from the surface wave from the impulse source (where the amplitude decreases as $\left.r^{-1 / 2}\right)$.

The magnetic field disturbances in the nearest zone might be connected, in general, with the longitudinal wave, because the surface wave have not time to develop yet.

In the simplest case, during the explosion with small depth, the longitudinal wave might be approximately considered as a wave, which keeps the central symmetry for the half-space, where it spreads. These results might be useful to the estimation of the contribution of the longitudinal wave at a large distance from the underground explosion too.

In this evaluation it is assumed, that the motion in the medium realizes along the radii from the centre, located on the medium surface. The area of motion is bounded by the flat free surface and by the semi-sphere, which is the front of the longitudinal wave, spreading from the origin of coordinates. This area and the point with coordinates $\left(x^{\prime}, y^{\prime}, z^{\prime}\right)$, where the magnetic field is evaluating, are show at Figure 3.3.3.1.

Vector of mass velocity $\vec{v}$ has direction, passing through the origin of coordinates. Its length, which is designated as $v$, depends on the distance from the coordinate centre $\rho=\left(x^{2}+y^{2}+z^{2}\right)^{1 / 2}$, and its components are the next:

$$
v_{\mathrm{x}}=v \frac{x}{\rho} ; \quad v_{\mathrm{y}}=v \frac{y}{\rho} ; \quad v_{\mathrm{z}}=v \frac{z}{\rho} \quad(3.3 .3 .1)
$$

Components of the vector $\vec{r}$ (directed from the point of the moving medium to the observation point) may be written as:

$$
r_{x}=x^{\prime}-x ; \quad r_{y}=y^{\prime}-y ; \quad r_{z}=z^{\prime}-z \quad(3.3 .3 .2)
$$

The velocity distribution might be represented as a product of the maximum value, which depends on $R$, and dimensionless function, which describes its change inside the motion area, depending on $\rho$ :

$$
v=v_{\text {m }}(R) \cdot f(\rho)
$$

Let's use the Biot-Savart's law to evaluate the arisen disturbances of the magnetic field.

Taking into account the fact, that the area of integration is the semi-sphere with radius $R$, we can receive the expression for the components of the magnetic field at the observation 
point on the base of equations (3.3.2.27) and (3.3.2.26). According to the international system of units, we receive the next expression for $H_{x}$ :

$$
\begin{aligned}
& H_{x}\left(x^{\prime}, y^{\prime}, z^{\prime}, R\right)=\frac{\sigma \mu_{0} v_{m}}{4 \pi} \int d z \int_{0}^{R} \int d y \quad \frac{\left(R^{2}-z^{2}\right)^{1 / 2}}{\left(R^{2}-z^{2}-y^{2}\right)^{1 / 2}} \int d x \frac{f(\rho)}{\rho} \cdot \mathbb{F}\left(\vec{r}, \vec{r}^{\prime}, \vec{H}_{0}\right) \\
& \text { 0 }-\left(R^{2}-z^{2}\right)^{1 / 2}-\left(R^{2}-z^{2}-y^{2}\right)^{1 / 2}
\end{aligned}
$$

where $\mathbb{F}\left(\vec{r}, \vec{r}^{\prime}, \vec{H}_{0}\right)=\frac{\left[\left(z^{\prime}-z\right)\left(z H_{0 x}-x H_{0 z}\right)-\left(y^{\prime}-y\right)\left(x H_{0 y}-y H_{0 x}\right)\right]}{\left[\left(x^{\prime}-x\right)^{2}+\left(y^{\prime}-y\right)^{2}+\left(z^{\prime}-z\right)^{2}\right]^{3 / 2}}$

Here it was used, that in the intemational system of units $\vec{B}_{0}=\mu_{0} \cdot \vec{H}_{0}$, where $\mu_{0}=4 \pi \cdot 10^{-7} \mathrm{H} / \mathrm{m}$. The expression for other components $H_{y}$ and $H_{z}$ might be received by means of cyclic permutation of the indexes and coordinates in the expression under the integral.

It is convenient to use dimensionless functions and coordinates. Let's divide the magnetic field by $H_{\text {oz }}$ component, and all distances by the distance $a$ from the explosion centre to the observation point. Then:

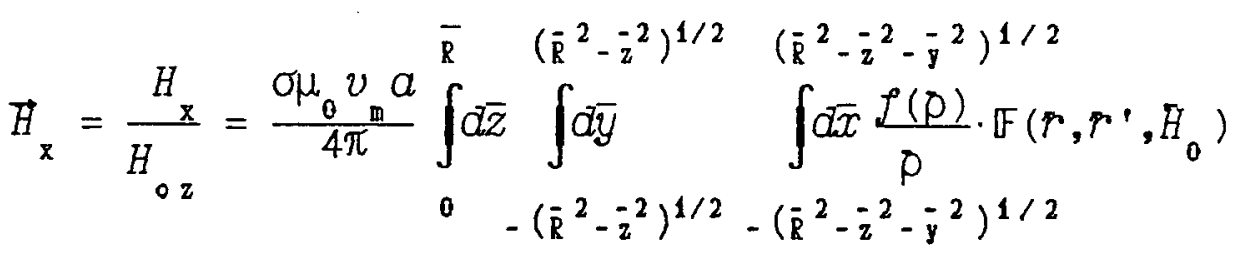

where $\mathbb{F}\left(r, r^{\prime}, H_{0}\right)=\frac{\left[\left(z^{\prime}-z\right)\left(z H_{0 x}-x\right)-\left(y^{\prime}-y\right)\left(x H_{0 y}-y H_{0 x}\right)\right]}{\left[\left(x^{\prime}-x\right)^{2}+\left(y^{\prime}-y\right)^{2}+\left(z^{\prime}-z\right)^{2}\right]^{3 / 2}}$

This expression might be rewritten as:

$$
H_{\mathrm{x}}=\frac{\sigma \mu_{0} a \cdot v(R)}{4 \pi} \mathbb{F}_{1}\left(R, x^{\prime}, y^{\prime}, z^{\prime}, H_{o x}, H_{o y}, \lambda, \gamma\right)
$$

where $\lambda$ and $\gamma$ are the parameters of $f(p)$, i.e. the parameters, which define the character of the mass velocity change in the motion area. If we know the dependence (3.3.3.6) we always may find the dependence of $H_{x}$ on time, supposing that the wave front $R$ moves with constant velocity $C_{1}$.

The change of the mass velocity behind the front might be 


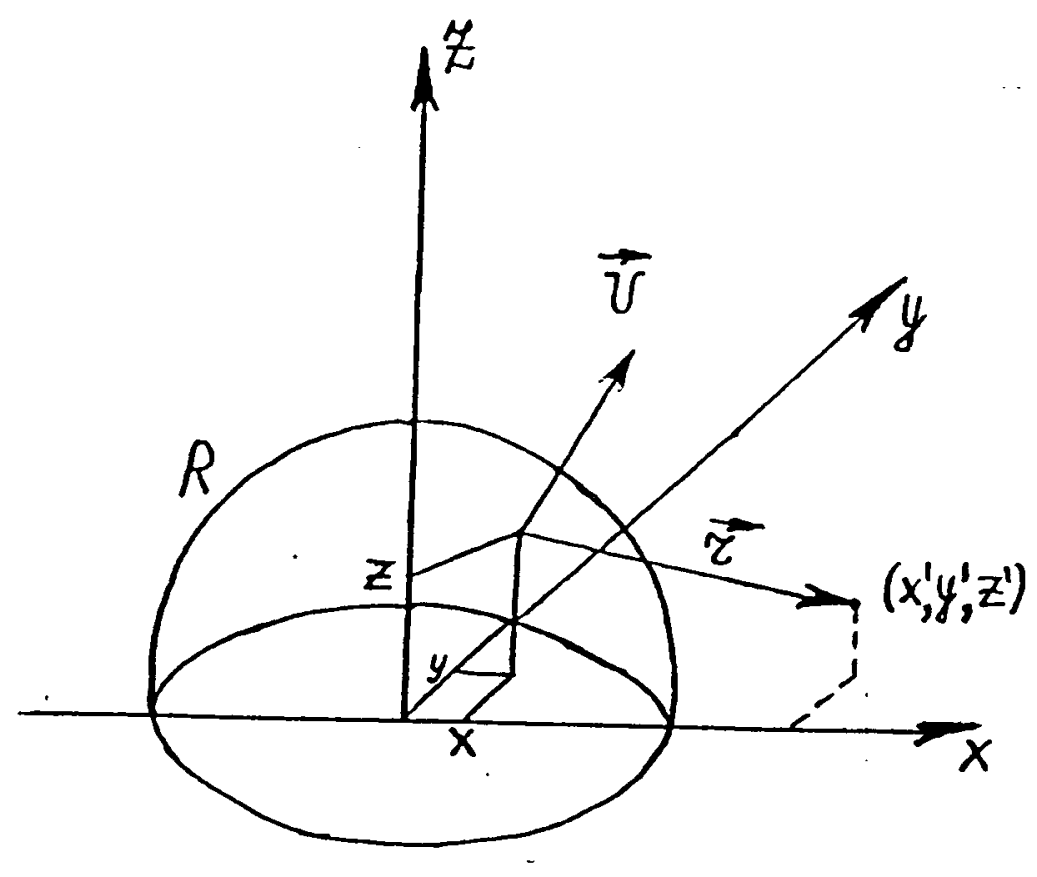

Figure 3.3.3.1. Coorditates for evaluation of the magnetic field. 
approximated by the sinusoid with some attenuation:

$$
f(\rho)=\sin \left[\frac{2 \pi}{\lambda}(\bar{R}-p)\right] \cdot \exp (-\gamma[(R-p)-\lambda / 4])
$$

Such kind of this function was chosen in order that its maximum value may be equal to 1 . If the moment of the wave arriving to the observation point as considered the beginning of time counting, than we receive the next expression for the argument of sine:

$$
\frac{2 \pi}{\lambda}(R-\rho)=\frac{2 \pi}{\lambda}(R-\rho)=\frac{2 \pi C_{1}}{\lambda} \cdot \frac{(R-\rho)}{C_{1}}=\frac{2 \pi}{T} \cdot t
$$

where $T$ is the wave period. The exponent power in equation $(3.3 \cdot 3.7)$ is

$$
-\gamma\left[(R-p)-\frac{\lambda}{4}\right]=-\gamma\left[(R-\rho)-\frac{\lambda}{4}\right]=-\gamma C_{1}\left(t-\frac{T}{4}\right)
$$

In whole, the dependence $f(t)$ is the next

$$
f(t)=\sin \left[\frac{2 \pi t}{T}\right] \cdot \exp \left(-\gamma C_{1}(t-T / 4)\right.
$$

We can choose the parameters on the base of the known record of the oscillations at some point. T corresponds to the wave period, and $\gamma$ might be found from the ratio of amplitudes of the positive and negative wave phases. In order to pass from $R$ to $t$ in the dependencies $(3.3 .3 .6)$ and $(3.3 .3 .7)$ we receive the follow formula

$$
t=\frac{a}{C_{1}}(\bar{R}-1)
$$

The dependence (3.3.3.5), represented above is considerably simplified and comes to the double integral, if we investigate only vertical component of the natural magnetic field $H_{o z}$. Let's use spherical coordinates during the integration of the Biot-Savart's formula, because in this case the current density has single component $J_{\varphi^{*}}$ Figure 3.3 .3 .2 shows the used coordinate system and the directions of the vectorial values. The natural magnetic field has its component only in that direction, the component of the velocity $\vec{v}$ is directed as a radius vector of the spherical coordinate system $\vec{\rho}\left(\rho^{2}=x^{2}+y^{2}+z^{2}\right)$. As it follows from the formula (3.3.2.26) in this case the current density has single component:

$$
j_{\varphi}=-\sigma \mu_{0} H_{c z} \cdot v \cdot \sin \theta
$$


Here the sign "minus" shows, that the direction of $J_{\varphi}$ is opposite to the direction of $\varphi$, which corresponds to the increase of the angle $\varphi$. In the Cartesian coordinates the components of $j_{\varphi}$ might be written as:

$$
\begin{aligned}
& j_{\varphi_{x}}=-\sigma \mu_{0} H_{o z} \cdot v \cdot \sin \theta \cdot \sin \varphi \\
& j_{\varphi_{y}}=-\sigma \mu_{0} H_{o z} \cdot v \cdot \sin \theta \cdot \cos \varphi \\
& j_{\varphi_{z}}=0
\end{aligned}
$$

The components of the vector $\vec{r}$ from the current element with coordinates $x, y, z$ in the Cartesian coordinate system and $\rho, \theta$ and $\varphi$ in the spherical system to the observation points with coordinates $x^{\prime}, y^{\prime}, z^{\prime}$, where the magnetic field is calculating, are described as:

Its length is

$$
\begin{aligned}
& r_{x}=x^{\prime}-\rho \cdot \sin \theta \cdot \cos \varphi \\
& r_{y}=y^{\prime}-\rho \cdot \sin \theta \cdot \sin \varphi \\
& r_{z}=z^{\prime}-\rho \cdot \cos \theta
\end{aligned}
$$

$r=\left(\rho^{2}+\left(x^{\prime 2}+y^{\prime 2}+z^{\prime 2}\right)-2 \rho\left[z^{\prime} \cos \theta+\sin \theta\left(x^{\prime} \cos \varphi+y^{\prime} \sin \varphi\right)\right]\right)^{\frac{1}{2}}$

In the case when the observation point is located on $\mathrm{X}$-axis at a distance $a$ from the origin of coordinates, we can write:

$$
\begin{aligned}
& r_{\mathrm{x}}=a-\rho \cdot \sin \theta \cdot \cos \varphi \\
& r^{\mathrm{y}}=-\rho \cdot \sin \theta \cdot \cos \varphi \\
& r^{\mathrm{x}}=-\rho \cdot \cos \theta \\
& r^{\mathrm{x}}=\left(a^{2}+\rho^{2}-2 a \rho \sin \theta \cdot \cos \varphi\right)^{1 / 2}
\end{aligned}
$$

For X-component $(\vec{J} \times \vec{r})_{\mathbf{x}}=j_{\varphi_{\mathbf{y}}} \cdot r_{\mathbf{z}}$ we receive, that

$$
(\vec{\jmath} \times \vec{r})_{x}=-\sigma \mu_{0} H_{o z} v \cdot \sin \theta \cdot \cos \theta \cdot \cos \varphi \cdot \rho
$$

From equation $(3.3 .2 .27)$ for $H_{x}$ in spherical coordinates, taking into account the evenness of the function under the integral by $\varphi$, we receive, that: 


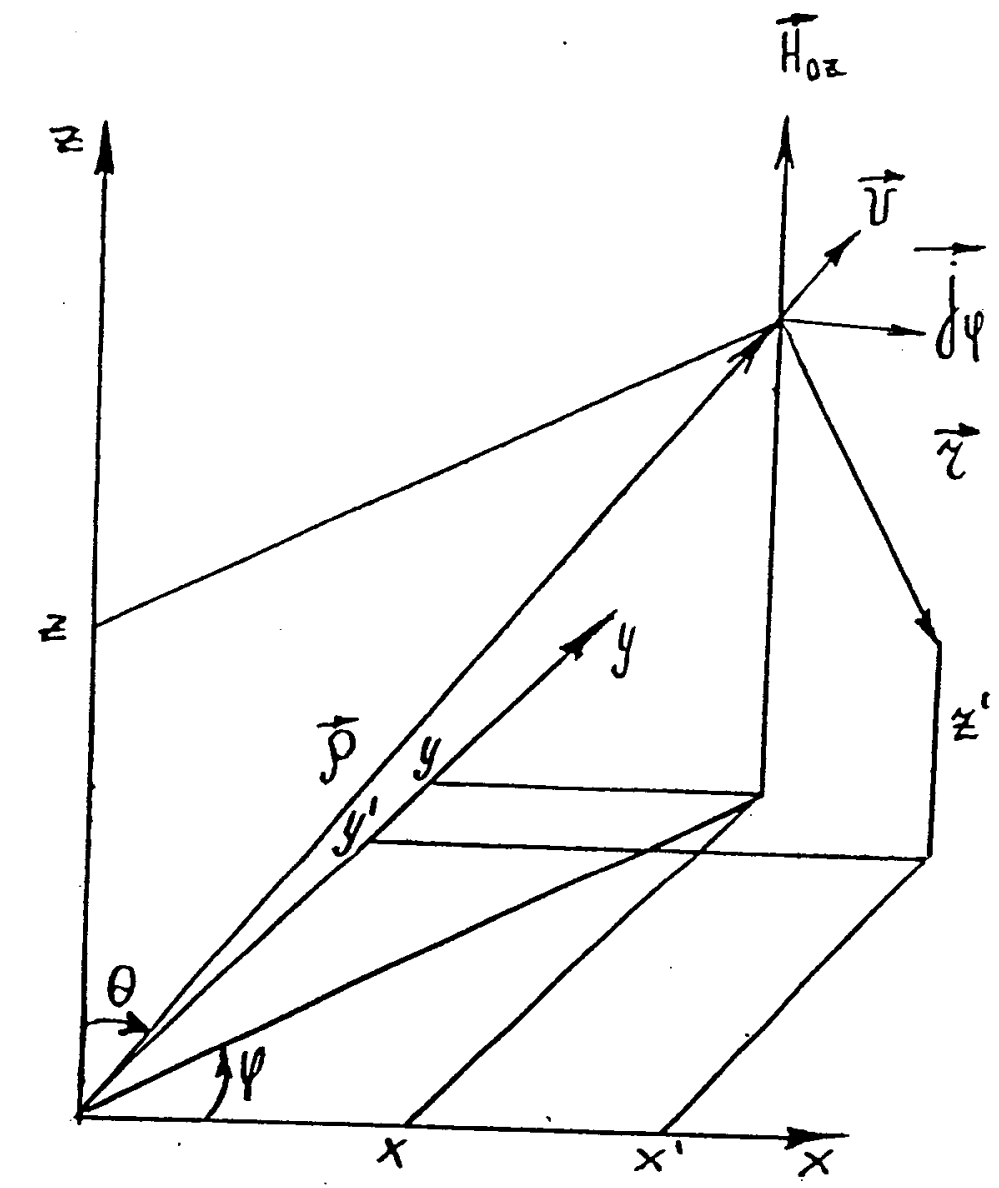

Figure 3.3.3.2. Coordinates for evaluation of the Biot-Savart's formula. 


$$
H_{x}=-\frac{\sigma \mu_{0} H}{2 \pi}{ }_{0 z} \int_{0}^{R} d \rho \int_{0}^{\frac{\pi}{2}} d \theta \int_{0}^{\pi} d \varphi \cdot \frac{\rho^{3} v \sin ^{2} \theta \cos \theta \cos \varphi}{\left(a^{2}+\rho^{2}-2 a \rho \sin \theta \cdot \cos \varphi\right)^{3 / 2}}
$$

This expression might be integrated by $\theta$ :

$$
\begin{gathered}
H_{x}=-\frac{\sigma \mu_{0} H_{0 z}}{\sigma \sqrt{2} \pi} \int_{0}^{R} v \cdot \mathbb{F}(n)\left(\frac{\rho}{a}\right)^{\frac{3}{2}} d \rho \quad(3.3 .3 .19) \\
\mathbb{F}(n)=n^{\frac{3}{2}} \int_{0}^{\pi}\left[\frac{3}{\left(1-\frac{\cos \varphi}{n}\right)^{\frac{1}{2}}}-8+\left(5+\frac{\cos \varphi}{n}\right)\left(1-\frac{\cos \varphi}{n}\right)^{\frac{1}{2}}\right] \frac{d \varphi}{\cos ^{2} \varphi}(3.3 .3 .20) \\
n=\frac{a^{2}+\rho^{2}}{2 a \rho}=\frac{1}{2}\left(\frac{\rho}{a}+\frac{a}{\rho}\right)
\end{gathered}
$$

If we use the expression (3.3.3.7) for the velocity and the empirical dependence for the velocity maximum, which is determined by two constants $A_{v}$ and $n_{v}$, then:

$v=v_{m} \cdot f(\rho)=A_{v}\left(\frac{q^{\frac{1}{3}}}{R}\right)^{n} \cdot e^{\frac{\Upsilon \lambda}{4}} \cdot \sin \left[\frac{2 \pi}{\lambda}(R-\rho)\right] \cdot e^{-\gamma(R-\rho)}$

After the substituting of this expression in (3.5.19) we receive, that

$H_{\mathrm{x}}=-\frac{\sigma \mu_{0} H_{0 \mathrm{z}} A_{\mathrm{v}} e^{\frac{\gamma \lambda}{4}}}{\sigma \sqrt{2} \pi}\left(\frac{q^{\frac{1}{3}}}{R}\right)^{\mathrm{n}^{\mathrm{R}}} \int_{0}^{\mathrm{R}} \sin \left[\frac{2 \pi}{\lambda}(R-\rho)\right] \cdot e^{-\gamma(R-\rho)} \mathbb{F}(n)\left[\frac{\rho}{a}\right]^{\frac{3}{2}} d \rho$

We use one of the empirical dependencies, which is shown by the dashed line at Figure 3.3.1., for the calculations

$$
v_{\mathrm{m}}=3.36 \cdot 10^{5}\left(\frac{q^{1 / 3}}{R}\right)^{1.6}
$$

Here $v$ is measured in $\mathrm{cm} / \mathrm{s}, q$ measured in $\mathrm{kt}\left(1 \mathrm{kt}=10^{6} \mathrm{~kg}\right)$, and $R$ measured in meters. For other parameters we use the next values: $C_{1}=3 \mathrm{~km} / \mathrm{s}, \sigma=0.25 \mathrm{~s} / \mathrm{m}, q=3.5 \mathrm{kt}, H_{0 z}=40 \mathrm{~A} / \mathrm{m}$, $\lambda=600 \mathrm{~m}$, and $\gamma=0.01 \mathrm{~m}^{-1}$.

If $H_{\mathrm{x}}$ is expressed in $m \gamma$, and $\alpha$ and $R$ are measured in meters, then we receive the next expression from equation $(3.3 .3 .23)$ 
$H_{\mathrm{x}}=-\frac{1.73 \cdot 10^{4}}{a^{0.6}(R / a)^{1 \cdot \sigma}} \int_{\mathrm{R}-\pi \lambda}^{\mathrm{R}} \sin \left[\frac{2 \pi}{\lambda}(R-\rho)\right] \cdot e^{-\gamma(R-\rho)} \mathrm{F}(n)\left[\frac{\rho}{a}\right]^{\frac{3}{2}} d\left(\frac{\rho}{a}\right)$

The lower Iimit of the integration was changed here. If $m=1$, the integration is carried out along wave length. Six variants with different distances to the observation point, where two values of $\gamma$ were used: $\gamma=0.01 \mathrm{~m}^{-1}$ and $\gamma=0$, were calculated on the base of this formula. The variants designation is represented in Table 3.3.3.1.

Table $3 \cdot 3 \cdot 3 \cdot 1$

\begin{tabular}{|c|r|l|r|r|}
\hline Variant & $\gamma \cdot a$ & \multicolumn{1}{|c|}{$\lambda / a$} & $a, m$ & $m$ \\
\hline I & 0 & 0.24 & 2500 & 1 \\
II & 0 & 0.15 & 4000 & 1 \\
III & 0 & 0.024 & 25000 & 1 \\
IV & 25 & 0.24 & 2500 & 1 \\
V & 40 & 0.15 & 4000 & 1 \\
VI & 250 & 0.024 & 25000 & 1 \\
\hline
\end{tabular}

The results of the calculations are shown at Figure 3.3.3.3 and 3.3.3.4 and in Tables 3.3.3.2, 3.3.3.3, and 3.3.3.4. The change of the maximum value $H_{x}$ modulus, expressed in $m \gamma$, with the change of the reduced distance from the explosion centre to the observation point is represented at Figure 3.3.3.5 in Iogarithmic coordinates. If we approximate these dependencies by the power function (as,$R^{-n}$ ), then in the case of $\gamma=0.01 \mathrm{~m}^{-1}$ the power index is $n=-1.36$, and if $\gamma=0, n$ is equal to -1.6 , i.e. this power index coincides with the power in the law of attenuation of the maximum of the mass velocity in the wave.

To compare this dependence with real data the experimental dependence from Figure 3.3.1.2 is shown at Figure 3.3.3.5 by solid line. Besides that, there is the point, corresponding to the explosion, for which the calculation was made, at this figure. We must say, that the value of the conductivity $0.25 \mathrm{~S} / \mathrm{m}$, used in the experimental data for this soil, is about ten times greater, than the tabular value for the soil with mean moisture (see Table 3.3.3.). So, the experimental results are 10-100 times greater, then the calculated values. Analogous 


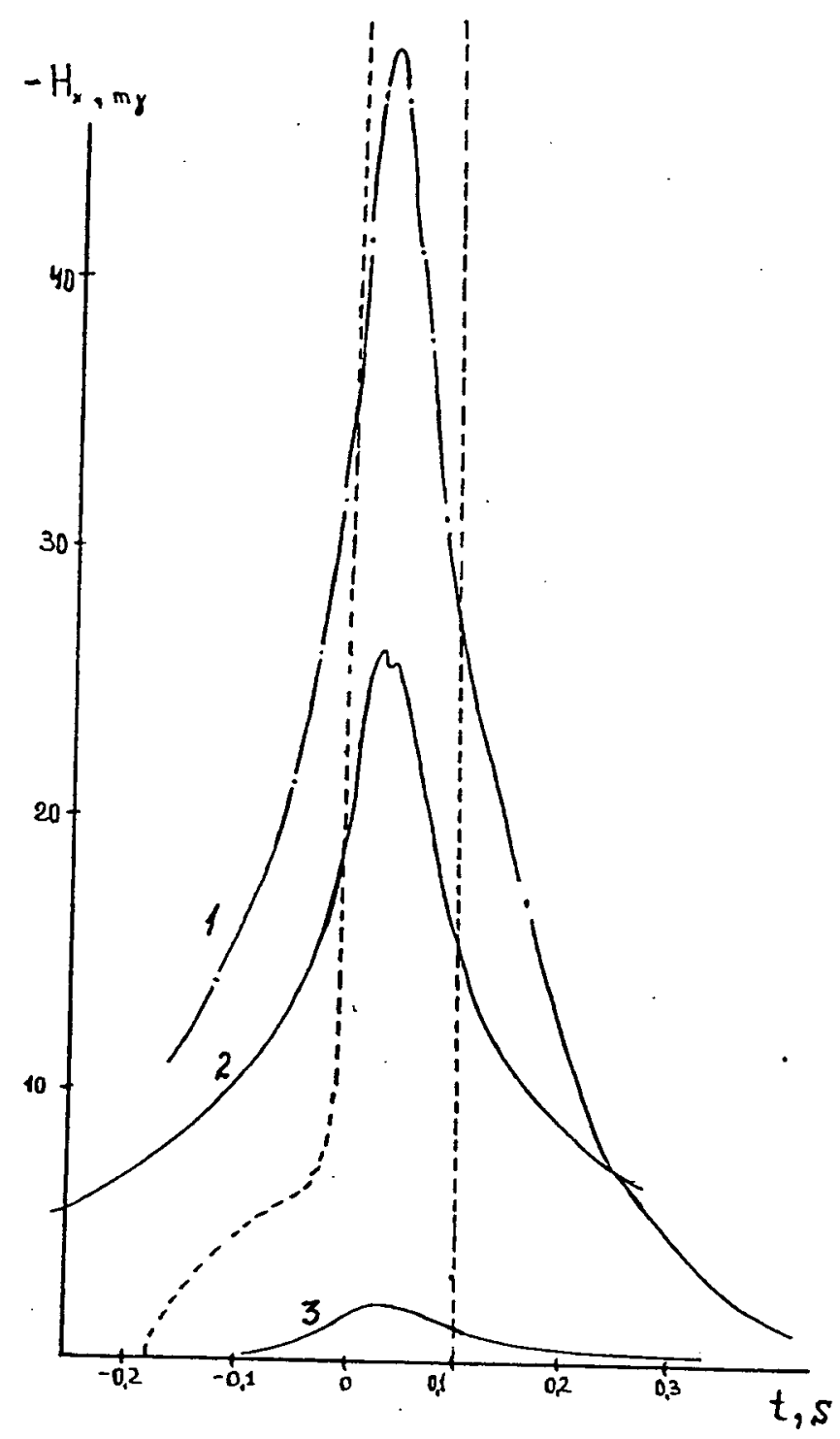

Figure 3.3.3.3. Magnetic $H_{\mathrm{x}}$ component at different distances from the epicenter. $\quad \gamma=0.01 \mathrm{~m}^{-1} ; \quad 1-a=2500 \mathrm{~m}$; 2- $a=4000 \mathrm{~m} ; 3-a=25000 \mathrm{~m}$. 


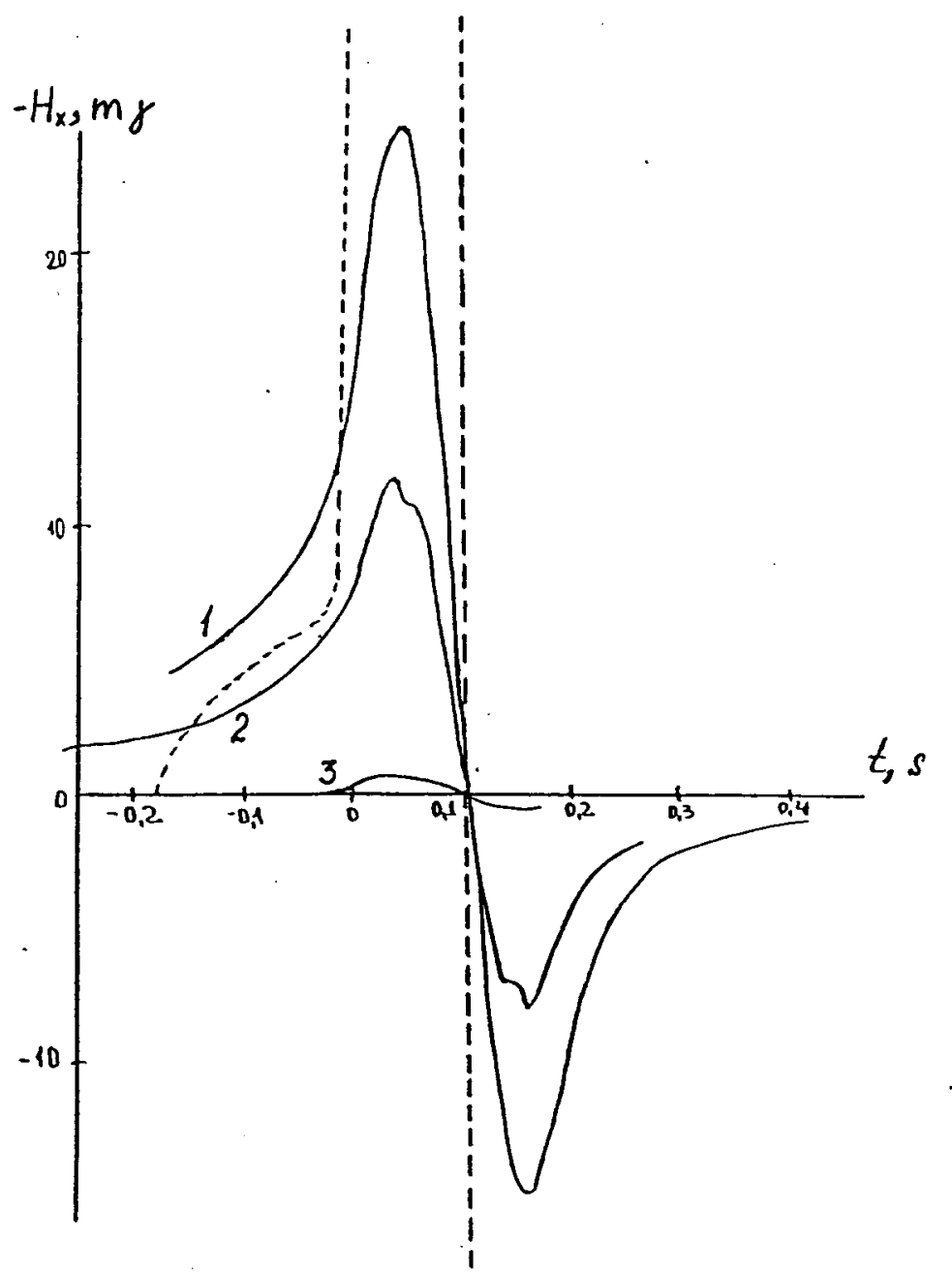

Figure 3.3.3.4. Magnetic $H_{x}$ component at different distances from the epicenter. $\quad \gamma=0 ; \quad 1-a=2500 \mathrm{~m}$;

2- $a=4000 \mathrm{~m} ; 3-a=25000 \mathrm{~m}$. 


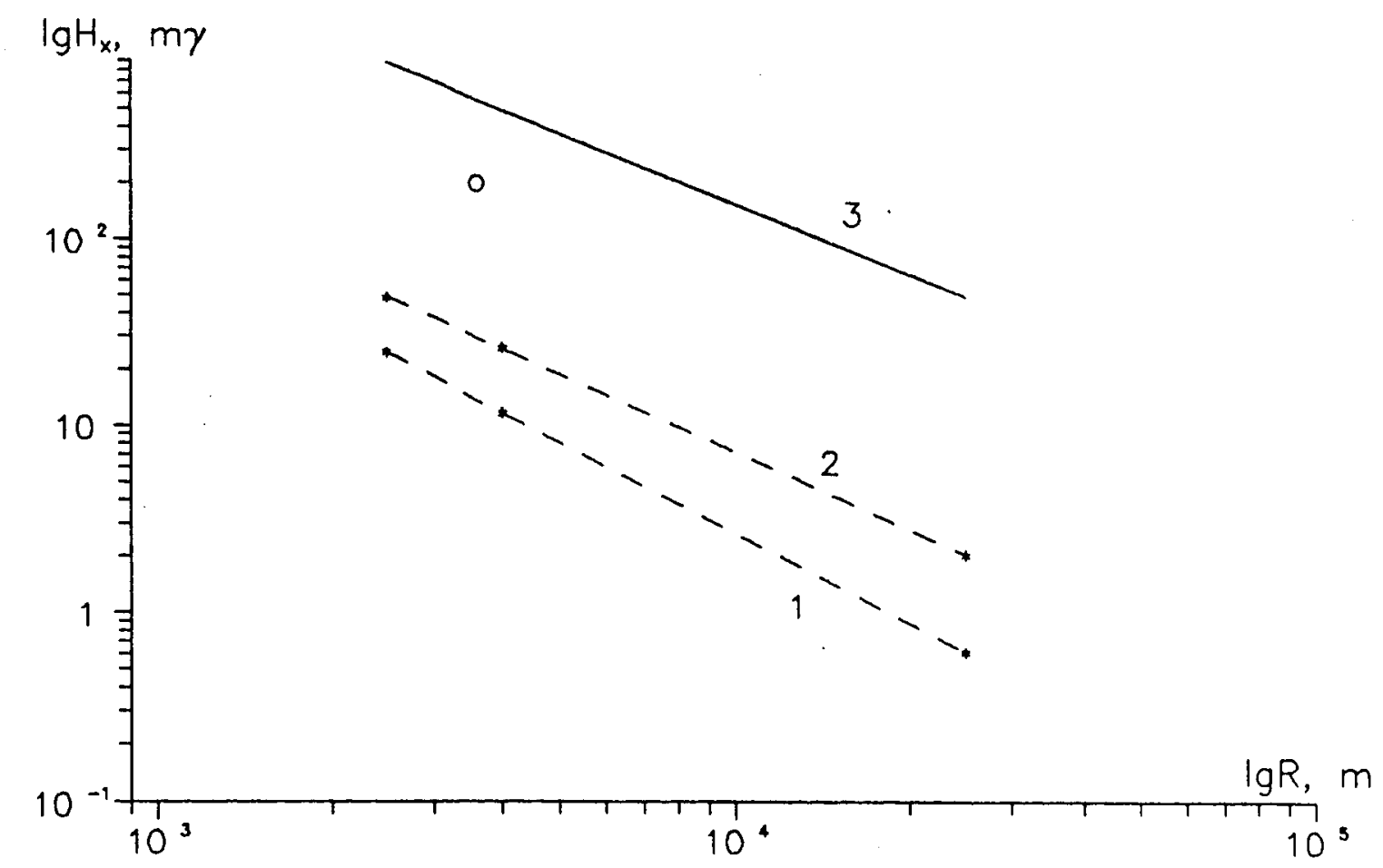

Fig.3.3.3.5. Max magnitude of the magnetic field (modulus) v.s. distance R, calculated by (3.3.3.25)

1 - for $\gamma=0$ (power is -1.6 ); $2-$ for $\gamma=0.01$ (power is -1.36 )

3- a best fit line from fig.3.3.1.2 ( $q=2.6 \mathrm{kt})$

and experimental magbitude $H_{x}$ for $q=2.6 \mathrm{kt}$. 
Table 3.3.3.2 $(a=2500 \mathrm{~m})$

\begin{tabular}{|c|c|c|c|}
\hline \multirow{2}{*}{$R / a$} & \multirow{2}{*}{$t, \mathrm{~s}$} & \multicolumn{2}{|c|}{$-H_{x}, m \gamma$} \\
\hline & & $\gamma=0.01 \mathrm{~m}^{-1}$ & $\gamma=0$ \\
\hline $\begin{array}{l}0.80 \\
0.90 \\
1.00 \\
1.01 \\
1.02 \\
1.03 \\
1.04 \\
1.05 \\
1.06 \\
1.07 \\
1.08 \\
1.09 \\
1.10 \\
1.11 \\
1.12 \\
1.13 \\
1.14 \\
1.15 \\
1.16 \\
1.17 \\
1.18 \\
1.19 \\
1.20 \\
1.30 \\
1.40 \\
1.50\end{array}$ & $\begin{array}{r}-0.167 \\
-0.083 \\
0.000 \\
0.008 \\
0.017 \\
0.025 \\
0.033 \\
0.042 \\
0.050 \\
0.058 \\
0.067 \\
0.075 \\
0.083 \\
0.092 \\
0.100 \\
0.108 \\
0.117 \\
0.125 \\
0.133 \\
0.142 \\
0.150 \\
0.158 \\
0.167 \\
0.250 \\
0.333 \\
0.417\end{array}$ & $\begin{array}{l}10.75 \\
17.47 \\
35.35 \\
41.30 \\
45.70 \\
48.40 \\
46.60 \\
40.95 \\
37.50 \\
31.10\end{array}$ & $\begin{array}{r}4.51 \\
7.18 \\
15.00 \\
17.50 \\
20.20 \\
23.90 \\
24.60 \\
22.80 \\
20.40 \\
13.20 \\
8.80 \\
-0.30 \\
-4.51 \\
-11.10 \\
-13.30 \\
-14.80 \\
-14.30 \\
-3.80 \\
-1.89 \\
-1.12\end{array}$ \\
\hline
\end{tabular}


Table 3.3.3.3 (a=4000 m)

\begin{tabular}{|l|c|c|c|}
\hline \multirow{2}{*}{$R / a$} & \multirow{2}{*}{$t, s$} & \multicolumn{2}{|c|}{$-H_{\mathrm{x}}, m \gamma$} \\
\cline { 3 - 4 } & & $\gamma=0.01 \mathrm{~m}^{-1}$ & $\gamma=0$ \\
\hline 0.80 & -0.270 & 5.37 & 1.72 \\
0.90 & -0.130 & 8.96 & 2.80 \\
1.00 & 0.000 & 19.89 & 7.22 \\
1.01 & 0.013 & 24.19 & 9.09 \\
1.02 & 0.026 & 26.12 & 10.60 \\
1.03 & 0.040 & 25.62 & 11.70 \\
1.04 & 0.053 & 23.56 & 10.70 \\
1.05 & 0.067 & 20.92 & 9.68 \\
1.06 & 0.080 & 18.37 & 6.91 \\
1.07 & 0.093 & 16.17 & 3.42 \\
1.08 & 0.107 & 14.42 & -0.24 \\
1.09 & 0.120 & 13.08 & -3.64 \\
1.10 & 0.133 & 12.14 & -6.22 \\
1.11 & 0.146 & & -7.15 \\
1.12 & 0.160 & & -8.12 \\
1.13 & 0.173 & & -7.19 \\
1.14 & 0.186 & & -5.91 \\
1.15 & 0.200 & & -3.48 \\
1.16 & 0.213 & & -2.90 \\
1.17 & 0.227 & & -2.49 \\
1.18 & 0.240 & & -2.18 \\
1.19 & 0.253 & & -1.93 \\
1.20 & 0.270 & 7.62 & \\
\hline
\end{tabular}


Table 3.3.3.4 $(a=25000 \mathrm{~m})$

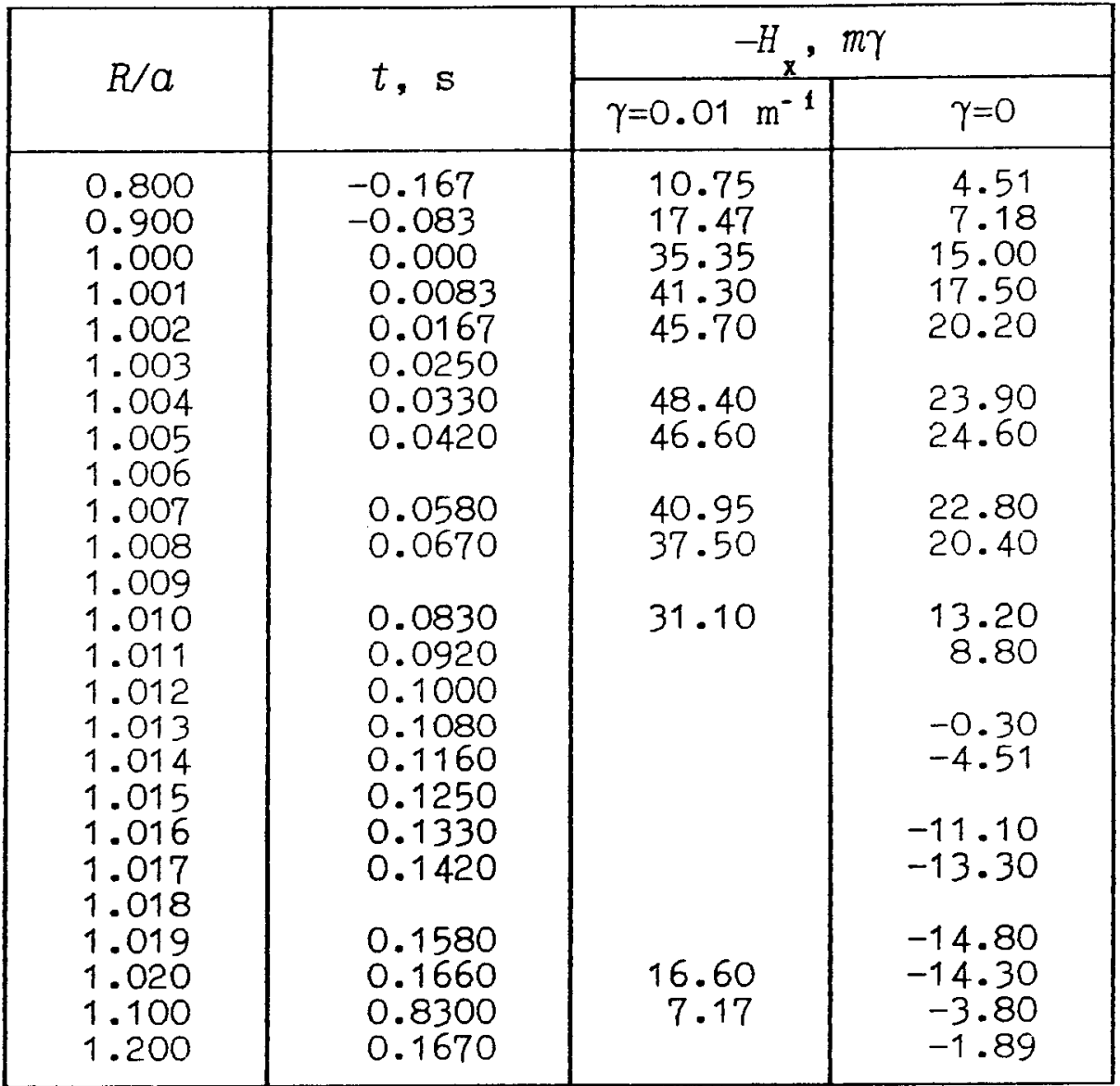

ratio was founded by Fleman, [1966] for the observed and calculated values of the magnetic disturbances during the earthquake, and also by Krotevich et al., [1977] for the waves on the water. The estimations for the Rayleight waves, represented by Gulielmi, [1986] and Gorbachev, [1987], give the values of the same order as the values, received here with the use of the analogous medium parameters.

The magnetic field disturbances take place outside the conductive moving medium (in the air) too. Because the considerable motion of the medium is observed in some bounded area more often, the use of the infinite periodical waves doesn't justify itself. The estimations, where the medium is divided into the areas of the current turns type, are useful 
here. The simplest case of this situation consists in the taking into account only vertical component of the extermal magnetic field and the motion, corresponding to the surface waves. In this case, the areas of the different phases in each time moment will induce the circular currents with the opposite directions. Each wave phase might be replaced by the equivalent turn. After that we can find the corresponding quasistatic disturbances of the magnetic field for the turns sum. In the case of single turn the disturbances will decrease with distance along the turn axis. This decrease is inversely proportional to the distance. In the case of two turns with opposite currents it is inversely proportional to the second power of the distance. Increase of the number of turns with opposite directed currents results in the power increase. Apparently, this dependence will be exponential in the utmost case.

To estimate the disturbances of the magnetic field we'll consider the surface waves at distances from the epicentre in the range of $10^{2} \mathrm{~m} / \mathrm{kt}^{1 / 3}<\bar{R}<10^{5} \mathrm{~m} / \mathrm{kt}^{1 / 3}\left(\bar{R}=R / \mathrm{q}^{1 / 3}\right)$. For the estimations in this range, it is possible to use the $R$ dependence of the maximum amplitude of the Rayleight wave as

$$
v=\frac{3.16 \cdot 10^{5}}{\bar{R}^{1} \cdot 5}
$$

where $v$ is measured in $\mathrm{cm} / \mathrm{s}, \vec{R}$ is measured in $\mathrm{m} / \mathrm{kt}^{1 / 3}$.

The wave period is about $0.2 \mathrm{~s} / \mathrm{kt}^{1 / 3}$, that corresponds to the reduced wave length $\bar{\lambda}_{r}=0.6 \mathrm{~km} / \mathrm{kt}^{1 / 3}$, when the surface wave velocity is $3 \mathrm{~km} / \mathrm{s}$. The motion attenuates with depth (along the vertical) according to the exponential law [Alfven et al., 1967] $v \cdot \exp \left(-z / z_{0}\right)$, where $z_{0} \cdot \frac{\lambda_{R}}{2 \pi\left(1-v^{2}\right)^{1 / 2}}$, and $\nu=\frac{C_{R}}{C_{t}}$ is the ratio of the Rayleght wave velocity and transversal wave velocity, that is of about 0.874-0.955. So, the characteristic depth is 2-3 times less, then the wave length. For the estimations we'll suppose, that the mass velocity in the wave is constant with depth in the layer with thickness of about $\lambda / 3$. Positive and negative wave phases will induce the currents with different directions. However, the whole compensation of the current isn't observed, because these phases have different amplitudes of the maximum velocities. 
It is convenient to carry out the calculations for two turns, which are the tours with the same circular section (with radius $a=\lambda / 6)$, where the currents are running. The ratio of the densities of these currents is analogous to the ratio of the maximums of the mass velocities of the positive and negative phases of the surface waves. At first, let's suppose that this ratio is equal to 2 .

First of all let's calculate the maximum value of the magnetic field in the direction of $H_{x}$ wave spreading, which will be reached at the boundary of the turn, corresponding to the positive phase of the surface wave. Its value is roughly the same, as this value for the straight line infinite current:

$$
H_{\mathrm{x}}=\frac{2 I}{c a}=\frac{2 j \pi a^{2}}{c a}=\frac{2 \pi a}{c} j
$$

The mass velocity in the surface wave will decrease with its spreading. Subsequently, the current density, which is described by the next formula, decreases too

$$
j=\frac{\sigma v B}{c}
$$

Substituting (3.3.3.28) to (3.3.3.27) and assuming that $a=m \lambda=m I v_{\mathrm{R}}$, where $m$ is the value, which must be chosen, we receive, that

$$
H_{\mathrm{x}}=2 \cdot B \cdot \sigma \cdot \mathrm{T} \frac{v \cdot C_{\mathrm{R}}}{C^{2}} \pi \cdot m
$$

When $m=1 / \pi$, i.e. $a=\lambda / \pi$, this formula coincides with the formula proposed by Gulielmi, [1986] for the infinite flat monochromatic Rayleight waves. The values of $v$ and $T$ must be chosen correctly for the comparison of these results with the results, received for the explosive waves.

On the base of the dependence $(3.3 .3 .26)$ we find, that

$$
H_{x}=\frac{2 \pi \cdot 316 \cdot 10^{3} a B \sigma}{c^{2}(\bar{R})^{1.5}}=\frac{2 \cdot 10^{6} a B \sigma}{c^{2}(\bar{R})^{1.5}}=\frac{q^{1 / 3}}{9 \cdot 10^{2}(\bar{R})^{1.5}}
$$

This dependence is shown at Figure 3.3.3.6 for 1,10 , and 100 $\mathrm{kt}$, when $\sigma=10^{8} \mathrm{~s}^{-1}, B=0.5$ gauss, $a=\lambda / \sigma, \lambda=0.6 \cdot 10^{5} q^{1 / 3}$ ( $q$ is measured in $\mathrm{kt}$ and $\lambda$ in $\mathrm{cm}$ ), $\overline{\mathrm{T}}=0.2 \mathrm{~s} / \mathrm{kt}^{1 / 5} C_{\mathrm{B}}=3 \mathrm{~km} / \mathrm{s}$.

As it is obvious from (3.3.3.30) in order to receive the similarity $H_{x}$ must be divided by $q^{1 / 3}$. It should be noted once again, that the theoretical estimations are about 100 times less, than the observed values. 
Now, let's estimate the change of the magnetic field disturbances in the explosion epicentre and on the vertical axis, directed from the epicentre. Let's approximate the currents, induced by the medium motion, by two circular currents

$$
I_{1}=\pi a^{2} \frac{\sigma B v_{\max }}{c}
$$

directed counter-clockwise, if we see to the Earth's surface from above, and

$$
I_{2}=0.5 I_{1}
$$

directed in the opposite side. Difference of the radii of these circular currents is equal to $\lambda / 2=0.3 \cdot 10^{5} q^{1 / 3}$, where $\lambda$ is measured in $\mathrm{cm}$, and $q$ is measured in kt. Certainly, that is a rough approximation, but it is necessary in order to take into account the wave attenuation. Substituting the corresponding values to equation $(3.3 .3 .31$ ) we receive, that

$$
I_{1}=\frac{3.16 \cdot 10^{13} \pi \delta B q^{1 / 3}}{c(\bar{R})^{1.5}}=1.65 \cdot 10^{11} \frac{q^{2 / 3}}{(\bar{R})^{1.5}}
$$

where $\sigma=10^{8} \mathrm{~s}^{-1}, B=0.5$ gauss, $I$ is measured in CGS units, $q^{\circ}$ is measured in $\mathrm{kt}$, and $R$ is expressed in $\mathrm{m} / \mathrm{kt}^{1 / 3}$.

The magnetic field disturbances in the epicentre $H_{c}$ are described as:

$H_{c}=\frac{2 \pi I}{c R}\left(1-\frac{1}{2} \cdot\left[1-\frac{\lambda}{2 R}\right]^{-1}\right)=\frac{0.345 \cdot q^{1 / 3}}{(\bar{R})^{2} \cdot 5} \cdot\left(1-\frac{1}{2} \cdot\left[1-\frac{\lambda}{2 R}\right]^{-1}\right)$

Here $H_{c}$ is measured in oersted, ' $q$ is measured in $\mathrm{kt}$, and $\bar{R}$ in $\mathrm{m} / \mathrm{kt}^{1 / 3}$. The direction of this disturbance is opposite to the direction of the vertical component of the natural Earth's field. We can see, that the disturbances values are very small. The magnetic field of the linear circular current is determined by the follow expression [Landau, 1982] in dependence on the turn radius $R$ and the coordinates of the observation point in the cylindrical coordinate system, where $r$ is the distance from the axis of symmetry, and $z$ is the distance from the turm plane. 


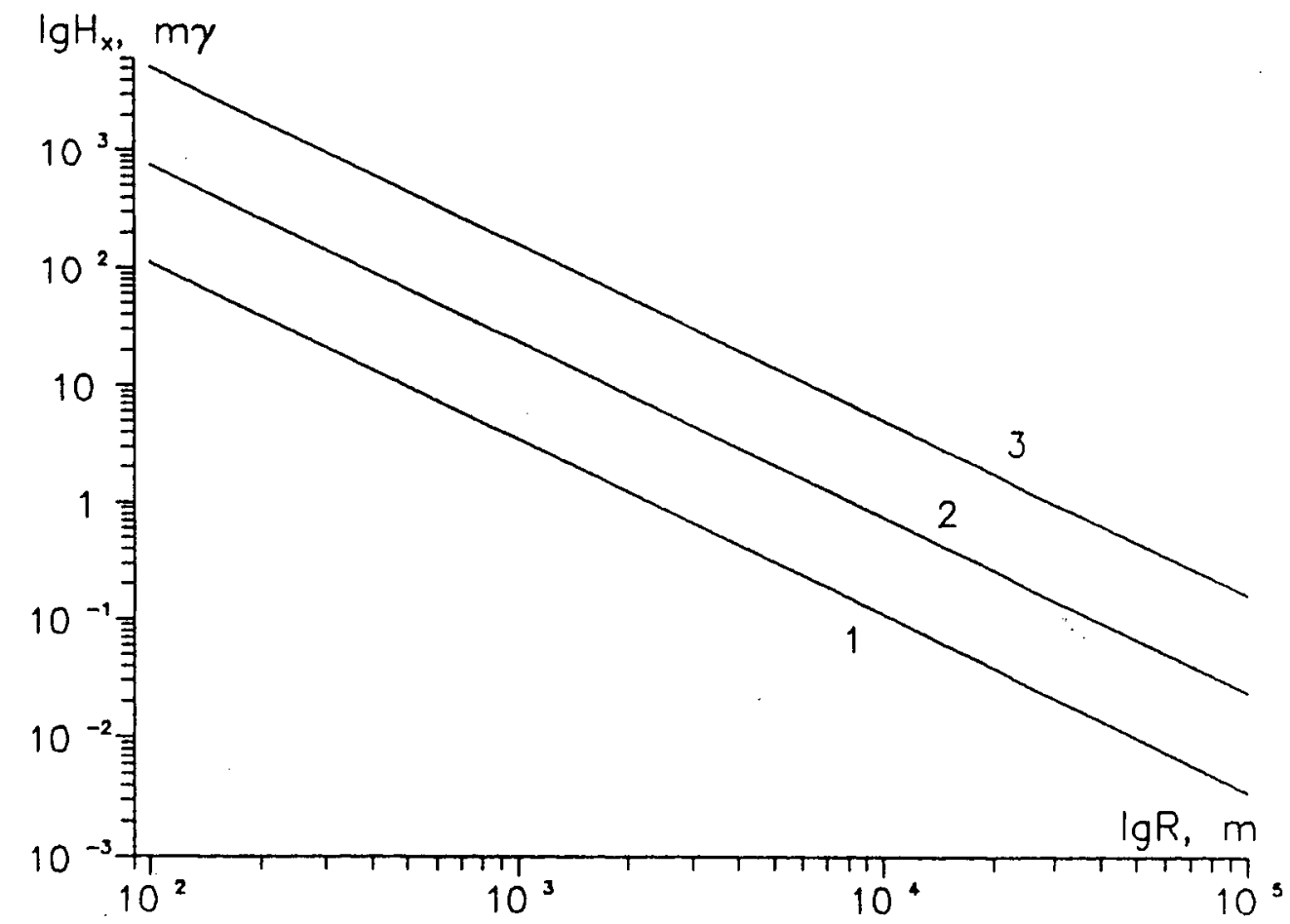

Fig.3.3.3.6. Max magnitude of the magnetic field

V.s. distance $R$, calculated by (3.3.3.30).

1 - for $q=1 \mathrm{kt} ; 2$ - for $q=10 \mathrm{kt}$;

3 - for $q=100 \mathrm{kt} ;(\sigma=0.01 \mathrm{~s} / \mathrm{m})$. 


$$
\begin{aligned}
H_{\mathrm{r}} & =\frac{I}{c} \frac{2 z}{r\left[(R+r)^{2}+z^{2}\right]^{1 / 2}}\left[\frac{R^{2}+r^{2}+z^{2}}{(R-r)^{2}+z^{2}} E-K\right] \\
H_{\mathrm{r}} & =\frac{I}{c} \frac{2}{\left[(R+r)^{2}+z^{2}\right]^{1 / 2}}\left[\frac{R^{2}-r^{2}-z^{2}}{(R-r)^{2}+z^{2}} E+K\right] \\
E & =\int^{\pi / 2}\left(1-\frac{4 R r \sin ^{2} \theta}{(R+r)^{2}+z^{2}}\right)^{1 / 2} d \theta \\
& \pi_{0}^{0} / 2 \\
K & =\int_{0}\left(1-\frac{4 R r \sin n^{2} \theta}{(R+r)^{2}+z^{2}}\right)^{-1 / 2} d \theta
\end{aligned}
$$

These expressions might be considerably simplified for the observation points, located on the turn axis $(r=0)$ :

$$
\begin{aligned}
& H_{r}=0 \\
& H_{z}=\frac{2 \pi R^{2} I}{c\left(R^{2}+z^{2}\right)^{3 / 2}}
\end{aligned}
$$

In the case of two currents we receive, that

$$
H_{z}=\frac{2 \pi R^{2} I_{1}}{c\left(R^{2}+z^{2}\right)^{3 / 2}}\left[1-\frac{1}{2} \cdot\left[1-\frac{\lambda}{2 R}\right]^{2} \cdot\left(\frac{z^{2}+R^{2}}{z^{2}+(R-\lambda / 2)^{2}}\right]^{\frac{3}{2}}\right]
$$

or, when $R \gg \lambda / 2$ :

$$
H_{z}=\frac{\pi R^{2} I_{1}}{c\left(R^{2}+z^{2}\right)^{3 / 2}}
$$

For our conditions $(3.3 .3 .33)$ it is the next:

$$
H_{2}=\frac{0.173 q^{1 / 3}(\bar{R})^{1 / 2}}{\left((\bar{R})^{2}+(\bar{z})^{2}\right)^{3 / 2}}
$$

Here, $H_{z}$ is measured in oersted, $q$ is measured in $k t$, and $\bar{R}$ and $\bar{z}$ are expressed in $\mathrm{m} / \mathrm{kt}^{1 / 3}$. It is obvious, that the field disturbances are small. But we must have in mind, that for the explosions with large power the greatest disturbanoes take place at the initial stages, when the strong motion, which can't be described by the ratios for the surface waves, is observed in the epicentral area. In this case it is necessary to carry out the additional analysis of all possible disturbances of the magnetic field. 
3.4. Disturbance of the atmosphere's electric field during the excavation explosions and underground nuclear tests.

First we consider the very low-frequency electric field in the atmosphere during the excavation explosions. The following section describe the results of the measurements that we curried out during both large-scale explosions and during a series small-scale explosions. But before we proceed further, it should be mentioned the work of Chermavsky [1955], who observed a disturbances of the atmosphere's electric field during the excavation explosions.

These large-scale industrial explosions was used for the building trade at Uzbekistan, Middle Asia. The masses of the high explosive charges for these events was about $1.6 \cdot 10^{6} \mathrm{~kg}$ and $1.8 \cdot 10^{6} \mathrm{~kg}$. The disturbance of the electric field was measured by a sensor in which an insulated and uncharged "Wilson plate" forming part of the earth's surface was connected to an electrometer ("induction-electrical measurement principle"). In this arrangement an induced electric charge is driven toward the other end of the plate-electrometer system where it produces a spreading of the electrometer filaments. Approximately equal magnitude of the field strength was observed during both of these large-scale explosions.

The author noted the typical features of the disturbances of the electric field: As a mule, a detonation of high explosives in the soil is accompanied by the electric field phenomena in the air. The initial spike of the electric field signal visually correlates to the dome's development and it is ahead of the arrival of seismic energy to the point of measurement. The greater is the mass of the explosive charge, the greater is the magnitude of initial spike of the electric field signal.

Appearance of the electric field impulses after the explosions in the air points to the presence of the intensive electric processes in the explosion centre. From the other side the processes of the disturbance and the deformation of the soil, induced by the underground explosion, are accompanied by 
a row of the mechanic-electrical phenomena, such as the shock polarization, the motion of the charged dislocations and other defects of the structure, piezoeffect, and the processes of the electrification, induced by the friction and rupture of the contacts between the particles of the soil body, which results in the electric charges separation.

So, during the explosions in the soil there is a row of the possibilities for micro separation, and, therefore, the disturbance of the electric field in the lower atmosphere layer.

3.4.1 Shape of the electric field signals and the dependence of the signals amplitude on the explosion depth.

A series of experiments was performed in which craters of centimeter to dekameter scale were formed by detonation of high explosive and the low-frequency electric fields in the air were measured [Adushkin, and Soloviev, 1988, 1989].

The mass of the explosive charges, $C$, in the series of experiments ranged from $0.023 \mathrm{~kg}$ to $12.8 \mathrm{~kg}$ and depth of burst $W$ ranged from 0 (detonation on the surface) to a depth $W_{k}$, corresponding to a contained explosion $\left(W_{k} \cdot C^{-1 / 3}=2-2.5\right.$ $\left.\mathrm{m} \cdot \mathrm{kg}^{-1 / 3}\right)$. The type of high explosive that we usually used in our experiments was trinitrotoluene (TNT).

A series of experiments in which craters were formed by the detonation of high explosive charges with mass $0.023 \mathrm{~kg}$, was carried out in sandy pool (soil moisture by weight or water content by weight is about 5\%). A series of experiments in which the mass of high explosive charges ranged from $0.4 \mathrm{~kg}$ to $12.8 \mathrm{~kg}$, was carried out in sandy loam (water content by weight ranged from $2 \%$ to $5 \%$ ). In some instances we locally increased the soil moisture by adding the water to the soil.

We also measured the atmospheric electric field generated by large industrial explosions. The masses of the high explosive charges for these events was about $2.6 \cdot 10^{6} \mathrm{~kg}$ (Uzbekistan, Middle Asia, 1983) and $3.2 \cdot 10^{4} \mathrm{~kg}$ (four explosions; Kirgizia, Middle Asia, 1987).

The disturbance of the vertical component of the 
potential gradient $E$ of the electric field was measured in the air by a "field mill" which senses the atmospheric electric field [Chalmers, 1967]. This instrument was installed on the ground surface at a distance $R$ from the epicentre $\left(R \cdot C^{-1 / 3}=\right.$ $\left.10-200 \mathrm{~m} \cdot \mathrm{kg}^{-1 / 3}\right)$.

An amplifiers and filter circuits were used during the measurements. We adjusted the gain to compensate for distance and signal strength. The equivalent output of the "field mill" was ranged from $2 \cdot 10^{-4} \mathrm{~V}$ per $\mathrm{V} \cdot \mathrm{m}^{-1}$ to $5 \cdot 10^{-3} \mathrm{~V}$ per $\mathrm{V} \cdot \mathrm{m}^{-1}$, since the magnitude of $E$ ranged up to $10^{4} \mathrm{~V} \cdot \mathrm{m}^{-1}$ at the closest $R$ $\left(R \cdot C^{-1 / 3}=10-20 \mathrm{~m} \cdot \mathrm{kg}^{-1 / 3}\right)$ and was comparable to the natural background at $R \cdot C^{-1 / 3}=100-200 \mathrm{~m} \cdot \mathrm{kg}^{-1 / 3}$.

The experiments described below were made only in periods of fair-weather in order to discount the meteorological factors such as clouds, thunderstorms, rain, strong wind, dust storms, etc. The fair-weather vertical electric field (the main potential gradient, which is positive when the field is directed downward) was $120-180 \mathrm{~V} \cdot \mathrm{m}^{-1}$ at the earth's surface. Typical fluctuations superimposed on the fair-weather electric field had amplitudes on the order of $25 \mathrm{~V} \cdot \mathrm{m}^{-1}$ and a period, peak to peak, on the order of $100 \mathrm{~s}$.

The low-frequency electric field generated by an explosion is a complex phenomenon which depends on the explosive mass $C$ and depth of burst $W$ as well as the material properties of the soil, water content, composition of the dust particles and their size distribution. Figure 3.4 .1 and Figure 3.4 .2 show typical records of the vertical electric field $E_{(}(t)$ that we obtained during our experiments [Adushkin and Soloviev 1988, 1989].

As a rule, $E(t)$ has the bipolar waveform in wet soil (water content by weight is about $5 \%$ in Figure 3.4 .2 , curve-b and it is about $15 \%$ in Figure 3.4 .1 , curve-a). The rise time for the initial spike is in the range 0.1 to $0.5 \mathrm{~s}$. The positive overshoot following the initial spike lasts for 30 to 1000 sec or more.

The magnitude of initial spike $E_{z}^{-}$as well as the magnitude of positive overshoot $E_{z}^{+}$depend on $W$. This dependence was studied during the small-scale experiments when $C$ was constant while $W$ ranged from $O$ to $W_{k}$. 


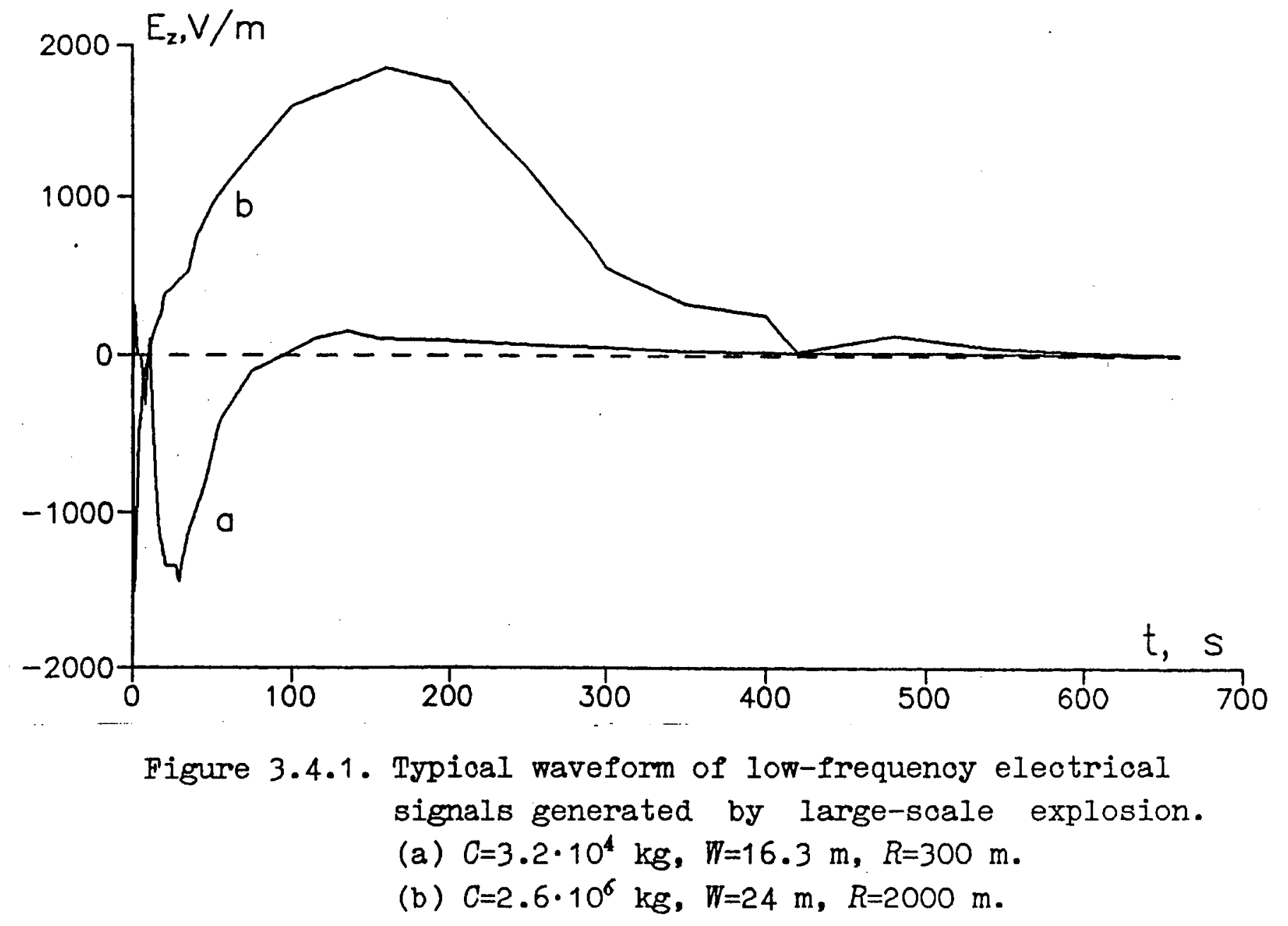




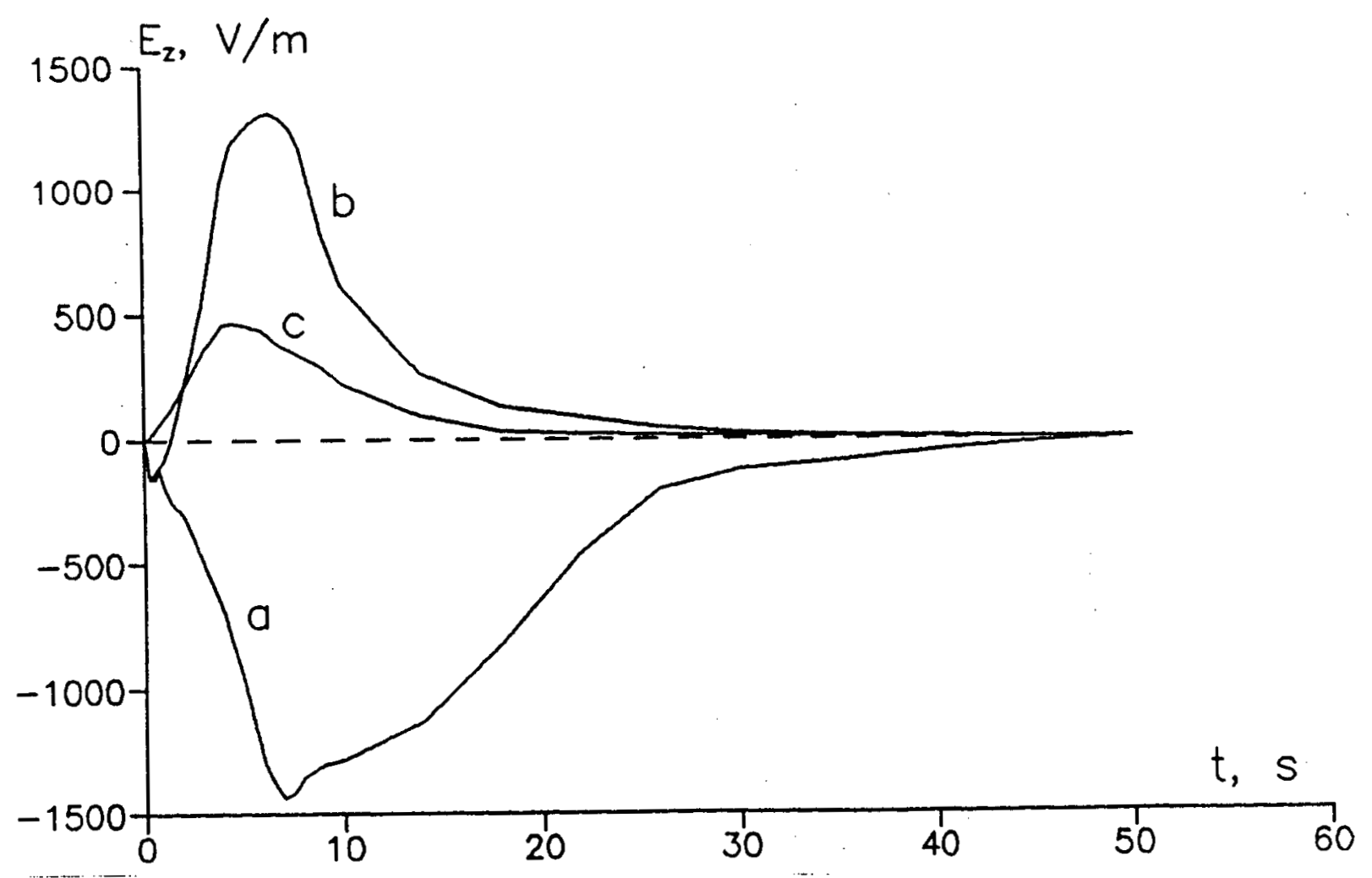

Figure 3.4.2. Typical waveform of low-frequenoy electrical signals, generated by small-scale explosion.

(a) Explosion in dry soil; $C=1.6 \mathrm{~kg}, W=0.05 \mathrm{~m}, R=41 \mathrm{~m}$.

(b) Explosion in wet soil; $C=1.6 \mathrm{~kg}, W=0.05 \mathrm{~m}, R=40 \mathrm{~m}$.

(c) Explosion in wide borehole without the stemming over an explosive; $C=1.6 \mathrm{~kg}, R=50 \mathrm{~m}$. 
Figure 3.4 .3 represents the records of $E_{z}(t)$ with fixed mass $C$ of the explosive charge and distance $R$ from the explosion epicentre to the point of $E_{z}$ measurements. We can see, that the signals $E_{z}(t)$ have a bipolar waveform but the amplitudes $E_{z}^{+}$and $E_{z}$ change in the dependence on the explosion depth $W$.

There is a value $W=W_{0}\left(W_{0} \cdot C^{-1 / 3} \simeq 0.2-0.3 \mathrm{~m} \cdot \mathrm{kg}^{-1 / 3}\right)$ corresponding to a maximum of magnitude of initial spike as well as overshoot. The magnitude of electric field signal increases with increasing $W$ from $O$ to $W_{0}$. As the depth of burst increases $\left(W>W_{0}\right)$, the magnitude of electric field signal begin to decrease and it becomes comparable to the natural background when the depth approximately equals to $W_{\mathbf{k}}$. Figure 3.4.4 shows the dependence of magnitudes $E_{z}^{*}$ and $E_{z}^{*}$ from $W$ when $C$ and $R$ were constant.

Successive steps of the explosion's development imaged with an instrumentation camera offer an opportunity to correlate the dome's development with the low-frequency electric field generated by the explosion. The rise time of the initial spike corresponds to the time elapsed from the beginning of the explosion to the time when the dome reaches its maximum height and intense cavity gas venting take place.

Curve-c in Figure 3.4 .2 shows the vertical electric field $E_{z}(t)$ that was generated by the high explosive in a wide borehole without stemming over the explosive. In this case only detonation products are vented to the atmosphere but no crater formation takes place. In this case only the positive phase of the electrical signal is observed.

It is significant that the detonation of high explosives above the surface of the ground (without crater formation) generates an electric field in the frequency range $10^{2}$ to $10^{6}$ $\mathrm{Hz}$ (see section 2).

In order to explain the observed time-variation of $E_{z}(t)$ we suggest that negative electric charges are mainly accumulated on soil particles while positive ones are collected on the products of the detonation.

In the experiments in the sandy soil, described above, the explosive charge had a spherical shape with diameter of 


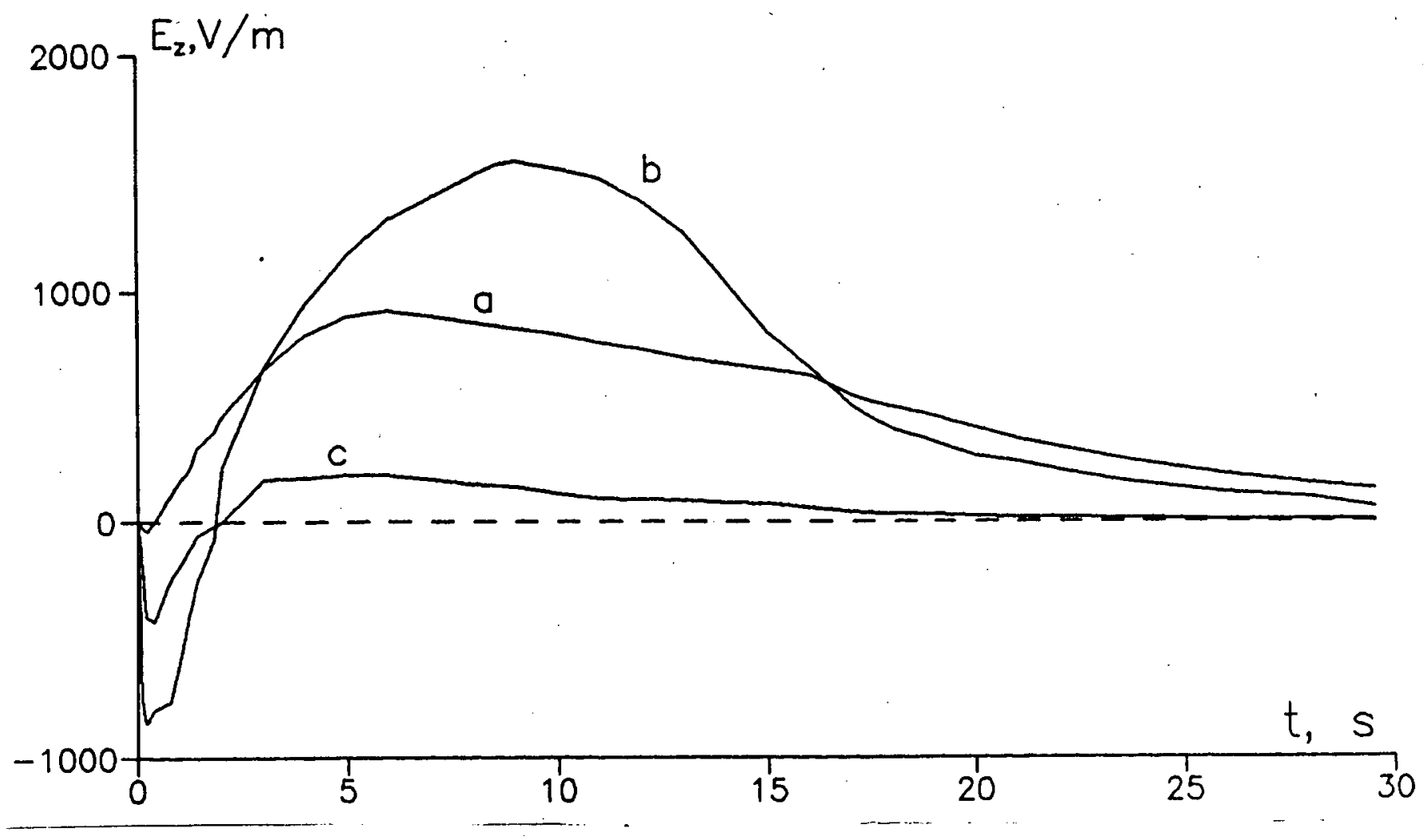

Figure 3.4.3. $E(t)$ from detonation of high explosive charges with mass $0.023 \mathrm{~kg}$ in sundy pool. (a) $\mathbb{W}=0, R=5 \mathrm{~m}$; (b) $-W=10.5 \mathrm{om}, R=5 \mathrm{~m} ; \quad(\mathrm{c})-W=13.7 \mathrm{~cm}, R=5 \mathrm{~m}$; 


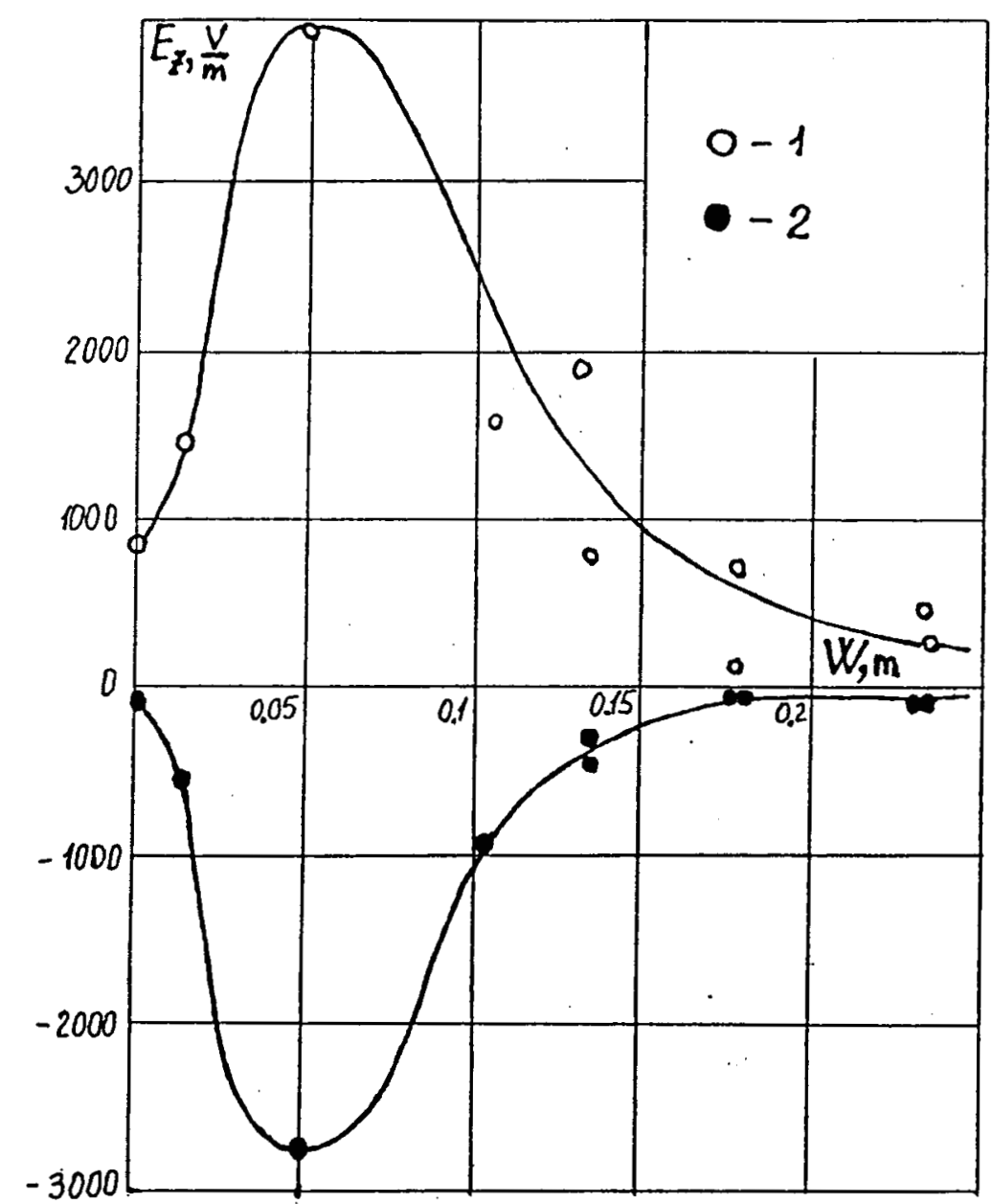

Figure 3.4.4. Magnitudes $E^{+}$and $E_{2}^{-} \nabla . s$. depth of burst $W$. $C=0.023 \mathrm{~kg}, \stackrel{\mathrm{z}}{R=5 \mathrm{~m} .}{ }^{2} 1-E_{\mathrm{z}}^{+} ; 2-E_{\mathrm{z}}^{*}$. 
$3 \mathrm{~cm}$ (the mass of the charge was equal to $23 \mathrm{~g}$ ). Figure 3.4 .5 demonstrates the influence of the charge shape on the signal amplitude $E_{z}(t)$. In both cases the charge mass and the depth of the charge centre were the same. Curve 1 corresponds to the explosion of the spherical charge with $d=3 \mathrm{~cm}$, and curve 2 corresponds to the explosion of the cylindrical charge with $d=1.5 \mathrm{~cm}$ and $l=7.7 \mathrm{~cm}$. From Figure 3.4 .5 we can see, that the magnitude of initial spike and the magnitude of positive overshoot from the elongated charge explosion are greater, than these values from the spherical charge, placed at the same depth $W$. But these magnitudes might correspond to the case of the spherical charge, located at the less depth. It should be noted, that in the considered experiment, the reduced depth of the charge location was greater, than the depth $W_{0}$, where the maximum signal $E_{z}$ is observed.

3.4.2 Influence of the soil properties on the electric field disturbances.

During the series of small-scale experiments we have been found that the shape of $E_{z}(t)$ changes if the soil water content is small enough and the percentage of dust particles in the soil is significant (Figure 3.4.2, curve-a). Taking into account this change of $E_{2}$ shape, a special series of experiments was carried out in dry dusty soil (sandy loam), in which the content of clay particles with dimensions ranging from 1 to $100 \mu \mathrm{m}$ was about 10-30\%. The shape of $E_{z}(t)$ also depended on the distance $R$ from the epicentre (Figure 3.4.6).

One can see that the shape of the registered signals for dry soil differs from explosions in wet soil. However, the initial spike is observed in all cases since it is closely associated with the generation of electric charges during dome development. On numerous occasions in the zone closest to the epicenter the signal polarity is negative. The positive overshoot is seen only at greater distances. This suggests that the dust cloud is polarized: the positive electric charges are concentrated in the upper part of the cloud, the negative ones in the bottom part of the cloud.

A cloud of dust particles simulated in the laboratory or 


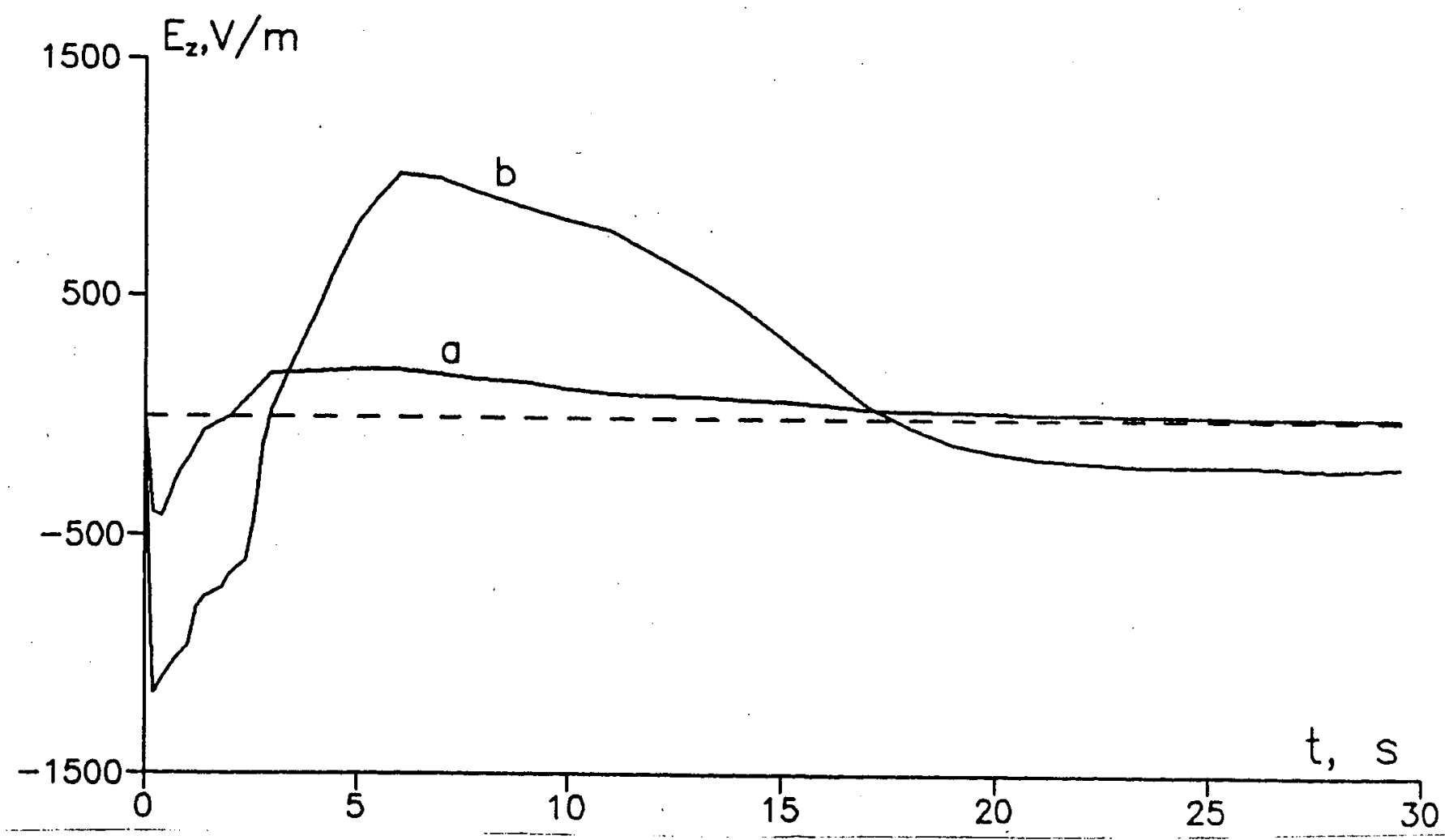

Figure 3.4.5. $E(t)$ from detonation of high explosive charges with mass $0.023 \mathrm{~kg}$ in sundy pool. $W=13.7 \mathrm{~cm} ; R=5 \mathrm{~m}$. (a) - spherical charge with $d=3 \mathrm{~cm}$;

(b) - cylindrical charge with $d=1.5 \mathrm{~cm}$ and $l=7.7 \mathrm{~cm}$. 


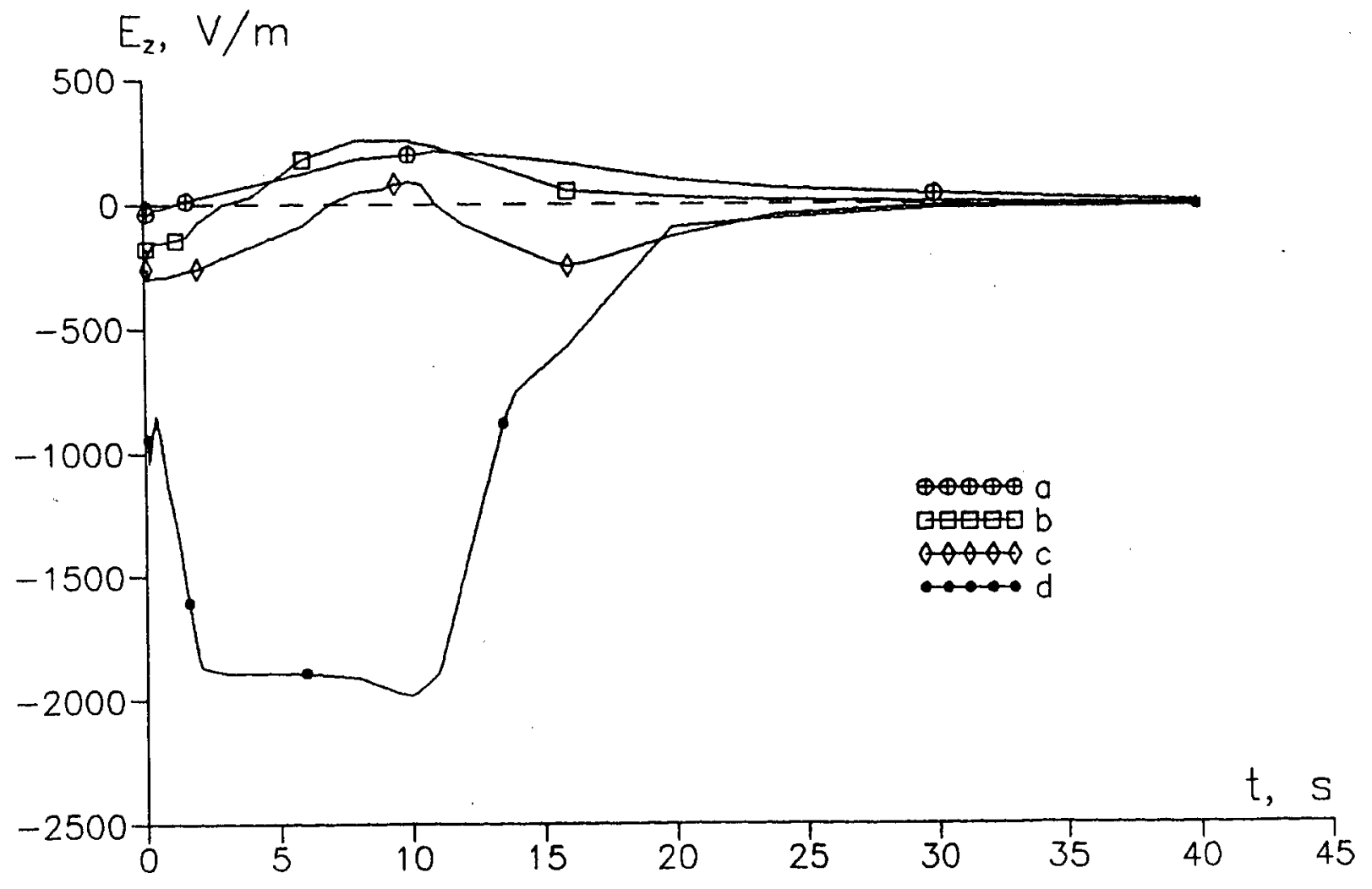

Figure 3.4.6. $E_{z}(t)$ generated by the explosion in dry, (a) - $R=66 \mathrm{~m} ;(\mathrm{b})-R=44 \mathrm{~m} ;(\mathrm{c})-R=37 \mathrm{~m} ;(\mathrm{d})-\mathrm{R}=25 \mathrm{~m}$. 
observed during dust storms or other natural processes in the atmosphere has an electric charge distributed throughout the volume of the cloud [Kamra 1972, 1973]. Both the polarity and magnitude of the electric charge on the dust cloud can change during sedimentation of the particles. Indeed, if coarse particles accumulate an electric charge of one sign while fine ones have the opposite charge, then the cloud becomes polarized by the relative motion of particles under the action of gravity and under the influence of the convective flow of gas. As the dust cloud rises, sedimentation of coarse particles causes a surplus of the opposite electric charge. The polarity and magnitude of the electric charge also depends on the chemical properties of the dust particles. For example, a cloud in which the main components are sand and clay produces a negative electric space charge. On other hand, the polarity of the electric space charge changes in time for experiments with samples of gypsum dust [Kamra 1973].

As it was shown by Frencel, [1949], Rulenko, [1985,] the particle electrification, arising because of the mupture of contacts between the particles, their destruction and so on, is explained by the difference in the electrochemical potentials $\Delta \varphi_{12}$ of the separated elements. The difference $\Delta \varphi_{12}$ depends on the chemical composition of the dust particles, their phase state, and the surface properties. After the contact mupture the charges $+q$ and $-q$ will appear on the particles [Rulenko, 1985, ]:

$$
|q|=\Delta \varphi_{12} \cdot C_{12}\left(1-e^{-\frac{\Delta t}{\tau}}\right)
$$

where $C_{12}$ is the initial capacity of the particles, $\Delta t$ is the contact duration, $\tau$ is the time of relaxation. Usually $\Delta t>\tau$, so $q \approx C_{12} \cdot \Delta \varphi_{12} * A \cdot \Delta \varphi_{12} \cdot r$, where $r$ is the particles radius. From the other side, when two heterogeneous particles are contiguous to each other, the condition of equilibrium comes to the fact, that the electric fields compensate each other at the place of this contact [Frencel, 1949]. The particles are in a metastable state, because their potentials aspire to be equal. But this aspiration might be realized when they are at a very small distance from each other (of about $10^{-7}+10^{-6} \mathrm{~cm}$ ). After the particle separation, their charges are proportional to the 
second power of their sizes. Laboratory investigations of the electrification of the volcanic ashes [Rulenko et al., 1986] allowed to find the empirical dependence of the average charge $Q$ of the particles from their diameter $d$ :

$$
Q \cdot a^{1.6 \pm 0.4}
$$

The particles with diameter $d=10 \mathrm{mkm}$ have a charge of about - 100e (where $e$ is the electron charge).

The motion of the dust-gaseous cloud in the atmosphere in the wind direction has a considerable influence on the shape of signal $E_{z}(t)$ and, especially, on the final phase. It results in the change of the mutual displacement of the dust-gaseous cloud and the electric field detector. Figure 3.4 .7 shows the records of $E_{(}(t)$, which were registered in the experiment, when the field mills were installed at the equal distances from the explosion epicentre in four perpendicular directions. The wind moved the dust-gaseous cloud with velocity of about $5 \mathrm{~m} / \mathrm{s}$ in the direction of one of the field mills. The records from this field mill is shown by a dashed line. It is obvious, that the negative phase of the signal is the same for all directions, whereas the positive phase depends on the direction and the velocity of wind. The change of sign of $E_{z}$ when $t=10+20 \mathrm{~s}$ is connected with the passing of the dust-gaseous cloud through the field mill. In future, when we'll treat the results of $E(t)$ measurements, we'll use the corrections of the distance $R$, which are connected with the wind influence on the motion of the cloud of the explosion products and dust.

3.4.3 Dependence of the amplitude of the electric field signals from the distance to the explosion epicentre.

It follows from the discussion above that after the detonation of a high explosive charge the high temperature products of the detonation and entrained surface material bear an electric charge. These electric charges are distributed throughout the volume of the dust cloud in a complicated manner.

In large explosions, the cloud of detonation products rapidly expands after detonation time, then it begins to rise 


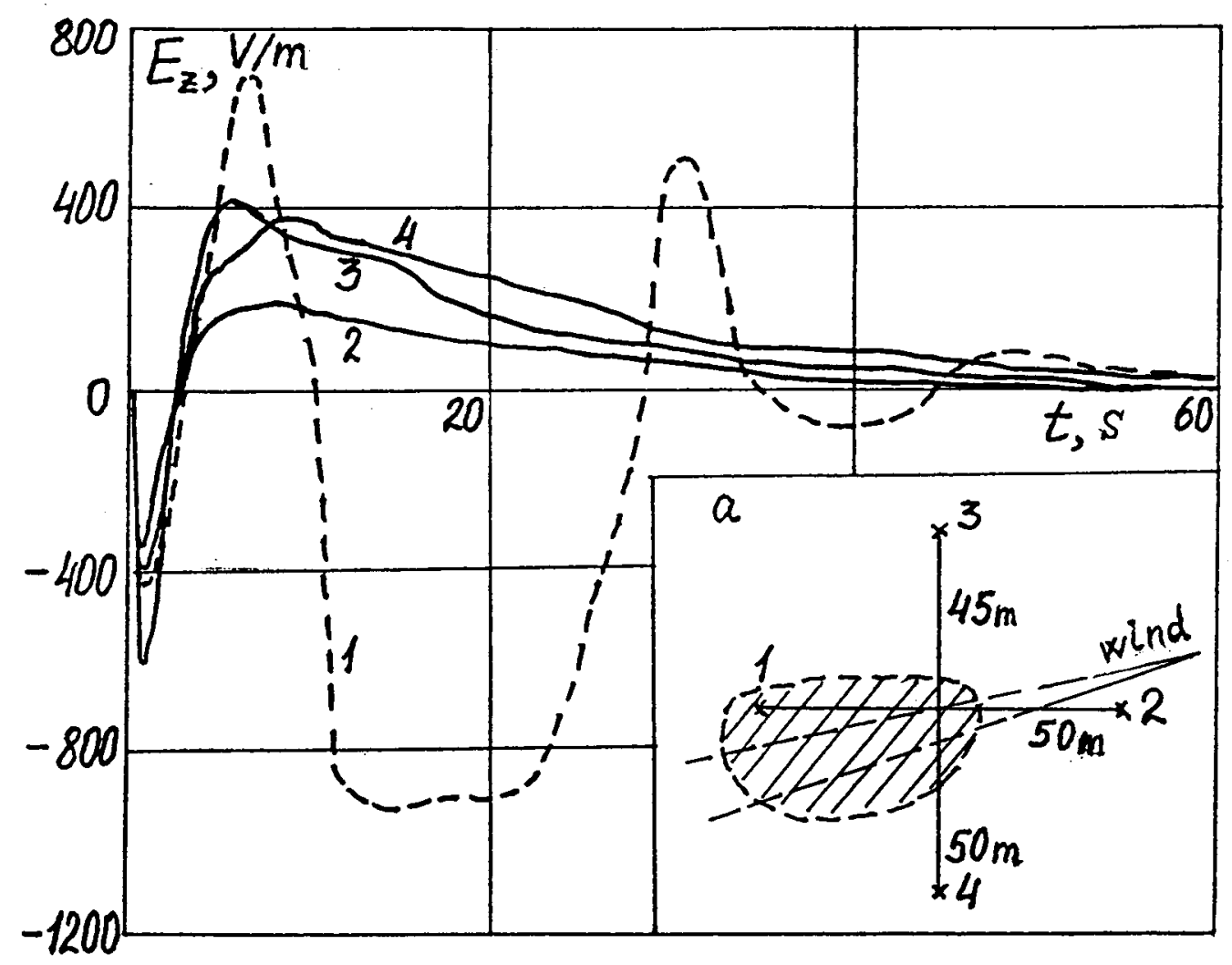

Figure 3.4.7. $E(t)$. from detonation of high explosive charges with mass $1.6 \mathrm{~kg}$ and $W=0.43 \mathrm{~m}$. a- scheme of the experiment. 
in the atmosphere. The convective movement in the central part of the cloud carries a great deal of dust upward from the ground surface. The rising cloud and associated electric space charge have a vertical cylindrical symmetry.

The potential gradient of the electric field in the neighborhood of the dust cloud is caused by the electric charge distributed in the cloud. We can estimate the magnitude of this electric charge by measuring $E_{2}(t, R)$, but this problem is not unique because the same $E_{z}(t, R)^{2}$ may be generated by different distributions of the electric charges. However, we can obtain an estimate of the electric charge magnitude if we make assumptions about the distribution of the electric charge in space.

We consider below some simple models of the disposition of the electric charge in space: A spherical charge $q$ placed at a height $H$ above the surface of the ground. Alternatively, a linear charge is given by $q=\sigma \cdot H$, where $\sigma$ is the linear charge density. Models may involve a combination of these two distributions. For example, the electric field produced by a spherical electric charge $q_{1}$ at height $H_{1}$ and a linear charge $q_{2}$, is determined by the relation

$$
E_{z}=\frac{q_{1}}{2 \pi \varepsilon_{0}}: \frac{H_{1}}{\left(R^{2}+H_{1}^{2}\right)^{3 / 2}}+\frac{q_{2}}{2 \pi \varepsilon_{0} H_{2}} \cdot\left(\frac{1}{R}-\frac{1}{\left(R^{2}+H_{2}^{2}\right)^{1 / 2}}\right) \text {, }
$$

where $R$ is the distance from the epicenter to the point of measurement (it is assumed that the ground is a conductor). Usually $q_{1}$ and $q_{2}$ have different sings. The four undefined parameters $q_{1}, q_{2}, H_{1}, H_{2}$ can be determined from the experimental value $E(t, R)$ on the basis of the nonlinear method of least-squares fitting for $E_{z}(t, R)$ in equation (3.4.1) [Bevington, 1967].

Sometimes we only need to consider the first term of equation (3.4.1). This is the case for the series of experiments in wet soil, where the variation of $E_{z}(t, R)$ is similar to those represented in Figure 3.4.1, curve $b$ and Figure 3.4.2, curve b. In this case equation $(3.4 .1)$ can be simplified to

$$
E_{z}=\frac{q}{2 \pi \varepsilon_{0}} \cdot \frac{H}{\left(H^{2}+R^{2}\right)^{3 / 2}}
$$


or represented as

$$
\frac{E_{2}}{E_{0}^{2}}=F\left(\frac{R}{H}\right)
$$

where $E_{0}=\frac{q}{2 \pi \varepsilon_{0} H^{2}}$ and $F\left(\frac{R}{H}\right)=\left(1+\frac{R^{2}}{H^{2}}\right)^{-3 / 2}$.

It should be noted that the model for the distribution of the electric charge in space is based on the assumption that electric charge is concentrated. This assumption is valid at distance $\frac{R}{r_{0}} \gg>1$, where $r_{0}$ is a characteristic size of the cloud, but it is not quite correct to use equation (3.4.1) or (3.4.2) at distance $\frac{R}{r_{0}}<1$ since the size of dust cloud should taken into account in the equation for electric field.

Figure 3.4.8 shows the dependence (3.4.3) which is associated with the experimental value $E_{z}(R)$ at the moment when $E_{z}(t, R)$ reaches its maximum value in the positive phase of the signal. For example, Figure 3.4 .9 shows $E_{z}(t, R)$ that was generated by the small-scale explosion and measured at various distances from the epicentre. The mass of the explosive charge is $C=12.8 \mathrm{~kg}$ and $W=0.5 \mathrm{~m}\left(W \cdot C^{-1 / 3}=0.214 \mathrm{~m} \cdot \mathrm{kg}^{-1 / 3}\right)$. The unknown parameters $q$ and $H$ are derived from a least-squares fit to the data at the moment $t=10 \mathrm{~s}$. The values of these parameters and the experimental value $E(R)$ at the same moment are given in the Table 3.4.1, where $R^{\frac{z}{\prime}}$ is the projection of the distance from rising dust cloud to the point of measurement on the earth's surface. To account for the dust cloud motion in the atmosphere, the mean wind speed and its direction was measured during our experiments.

One can use equation (3.4.1) or more complicated models of the disposition of electric charges when the experiments are made in dry soil and the variation of $E_{z}(t)$ is similar to the ones represented in Figure 3.4.3. However, the number of measurements of $E_{\mathrm{z}}(t, R)$ must exceed the number of unknown parameters in the equation for $E_{z}(R)$ in order to get reliable values for these parameters.

At Figure 3.4 .10 we can see the dependencies $E(R)$ and $E^{*}(R)$ calculated on the base of the expression $(3.4 .1)$. the experimental values of $E_{z}$ and $E_{z}^{*}$, received during the measurements at different distances from the explosion 


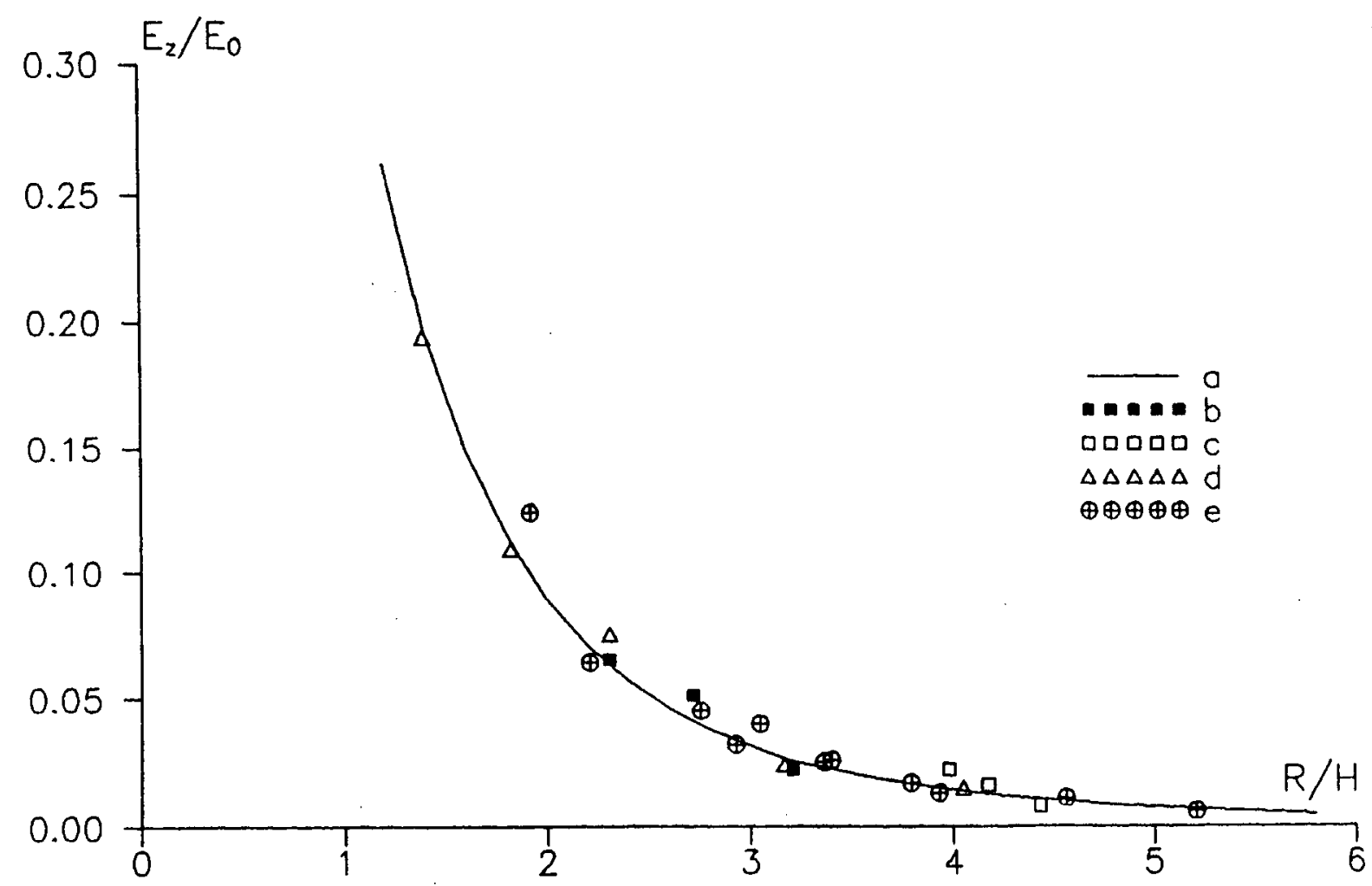

Figure 3.4.8. Dimensiomless potential gradient v.s. dimensionless distance for the explosion in wet soil. (a) - Relation (3.4.3); (b) - $\mathrm{C}=0.023 \mathrm{~kg}$;

(c) - $C=0.4 \mathrm{~kg} ;(\mathrm{d})-C=1.6 \mathrm{~kg} ;(\mathrm{e})-C=12.8 \mathrm{~kg}$. 


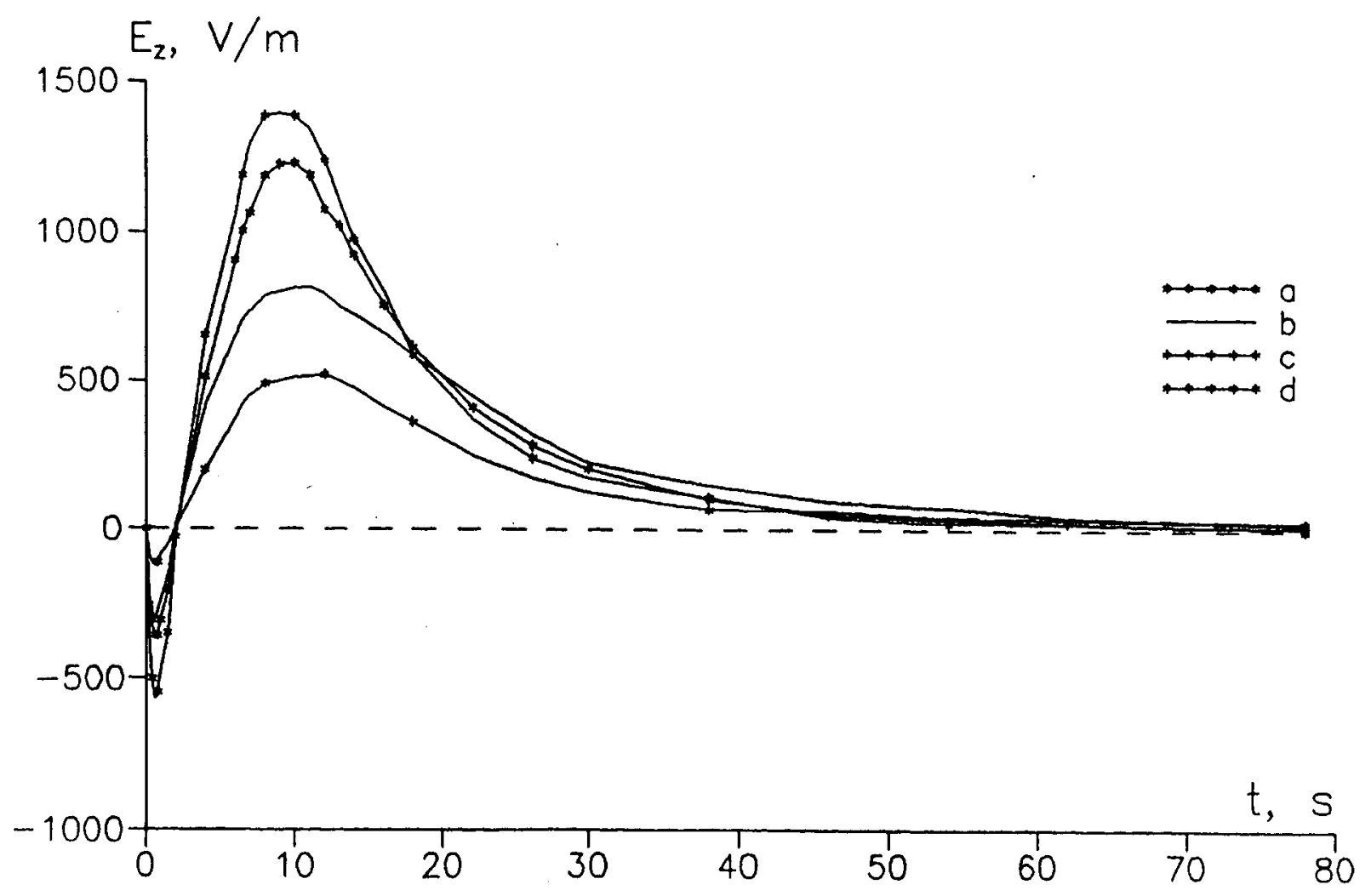

Figure 3.4.9. $E_{2}$ generated by the small-scale explosion at various distances from the epicenter. $C=12.8 \mathrm{~kg} ; \quad W=0.5 \mathrm{~m} ;$ (a) $-R=95 \mathrm{~m}$; (b) $-R=85 \mathrm{~m} ;(\mathrm{c})-R=75 \mathrm{~m} ;(\mathrm{d})-R=65 \mathrm{~m}$. 


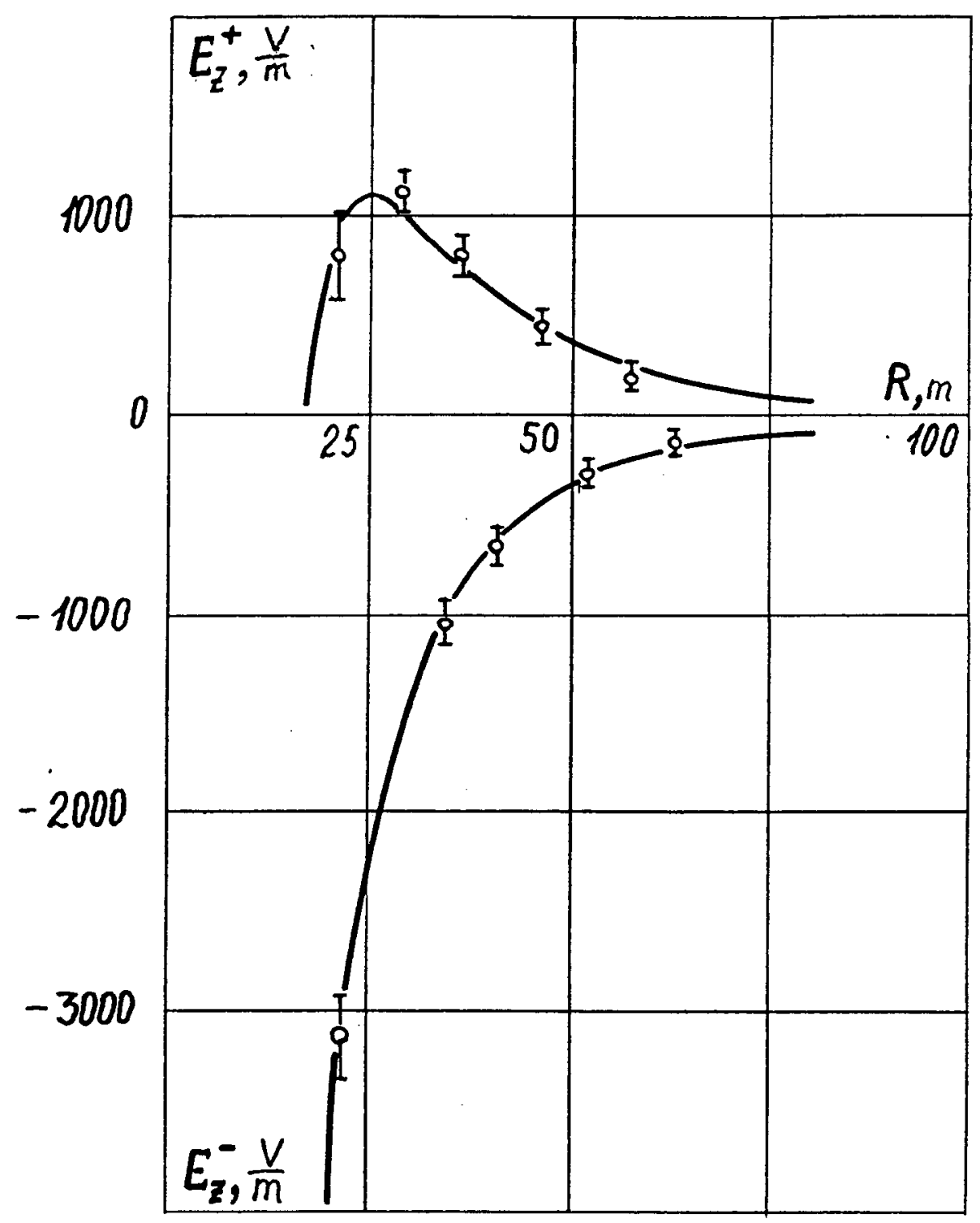

Figure 3.4.10. $E(R)$ and $E^{+}(R)$ for explosion in dry dusty soil. $C=1.6 \mathrm{~kg}, W=0.45 \mathrm{~m}$. The correction for dusty cloud motion in the atmosphere take into account. 
Table3.4.1. Experimental value $E_{z}(t)$ and computed parameters

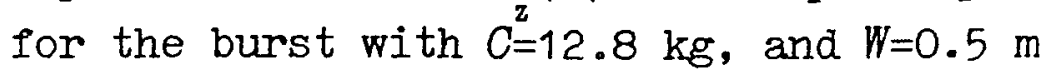
$\left(W \cdot C^{-1 / 3}=0.214 \mathrm{~m} \cdot \mathrm{kg}^{-1 / 3}\right)$ at the moment $t=10 \mathrm{~s}$.

\begin{tabular}{ccccccc}
\hline $\mathrm{E}_{\mathrm{z}}(\mathrm{t}), \mathrm{V} / \mathrm{m}$ & $R, \mathrm{~m}$ & $R^{*}, \mathrm{~m}$ & $q \cdot 10^{5}, \mathrm{C}$ & $\mathrm{H}, \mathrm{m}$ & $\frac{R^{\prime}}{\mathrm{H}}$ & $\frac{\mathrm{E}}{\mathrm{E}_{\mathrm{o}}} \cdot 10^{2}$ \\
\hline 1395 & 65 & 53.0 & & & 2.76 & 4.54 \\
1230 & 75 & 58.6 & $63 \pm 3$ & $19.2 \pm 0.9$ & 3.05 & 4.0 \\
810 & 85 & 65.3 & & & 3.4 & 2.64 \\
520 & 95 & 72.8 & & & 3.8 & 1.69 \\
\hline
\end{tabular}

epicentre, are shown at this figure too.

Let's calculate the evolution of the electric signals in time on the base of the simple one-dimensional model of the dome's development including the products of detonation, but without concrete definition of the mechanism of the electrification of the soil particles [Aduchkin at al., 1990]. To calculate the electric field strength far from the explosion epicentre we used the above model for distribution of the electric charge in space. Then, the vertical component of the electric field strength on the soil surface at a distance $R$ from the explosion epicentre is described by (3.4.1), but parameters $q_{1}=-Q_{1}, q_{2}=Q_{2}, H_{1}, H_{2}$ are a function of the time. The field of the electric charges, induced in the Earth under the condition of its ideal conductivity, is taken into account in (3.4.1).

The heights of the dome lifting and the dust-gaseous cloud lifting might be approximately described by function $H_{1}=H_{2}=U_{H} t-g_{0} t^{2} / 2$, where $U_{H}$ is the initial velocity of the soil, $g_{0}$ is the value much greater, than the gravitational acceleration $g$. In general, it is connected with the resistance of the air to the soil motion. To make an estimate of $g$ 。 we used the successive steps of the explosion's development imaged with the instrumentation camera.

Assuming, that the linear density of the charge $\sigma$ for 
Iinear charge $Q_{2}=\sigma H$ is constant at the lifting phase, we have, that:

$$
Q_{2}=-Q_{1}=\frac{Q_{0} H_{2}}{H_{\mathrm{m}}}=Q_{0} \frac{U_{H} t-g_{0}^{2} t / 2}{H_{\mathrm{m}}},
$$

where $Q_{0}$. is a charge at the height of the greatest lifting $H_{m}$. It is believed, that $g_{0}=g$ in the expression for $H_{1}$ at the phase of the fragmented soil drop. Then the soil charge is $Q_{1}=-Q_{0}$. The charge $Q_{2}$ is determined by the former formula, where $H_{2}$ is reduced because of the settling of the dust particles. We must take into account the forces of the air resistance, acting on the charged particles of dust to describe this process.

Let's assume, that the precipitation velocity and the particles size $a$ are small, i.e. Re $<1$. Then, the equation of motion of particles with mass $m(a)$ and charge $q(a)$ might be written, as

$$
\frac{d V}{d t}=g-\frac{V-U}{\tau}+g \frac{E}{\mathrm{~m}}, \quad \tau=\frac{m}{6 \pi \eta n a},
$$

where $V$ is the particle velocity, $U$ is the gas velocity and $E$ is the strength of the geolectric and self-coordinated electric field of the charged particles and the charges, induced in the Earth, and $\eta$ is the viscosity coefficient for the air. This consideration is simplified, because it doesn't take into account the forces of the floatability of the dust-gaseous cloud and the size distribution of the dust particles. But it gives an opportunity to describe the change of the field strength in time during small-scale explosions in the soil with small variation of the particles sizes from the average value in the sand, for example. With use of the next values: $a=20 \mu \mathrm{m}$ for the dust particle sizes, $q=100 \mathrm{e}$ (where $e$ is the electron charge) for the particle charge, and $E_{p}=30 \mathrm{kV} / \mathrm{m}$ is a breakdown electric field strength in the air, we receive, that $\left(q E_{p}\right) / m=0.7 \mathrm{~m} / \mathrm{c}^{2}<g$, i.e. we can neglect the influence of the electric field on the motion of the particles with such sizes. Nevertheless, the influence of the electric field on the motion might be considerable for the small-dispersive dust with large specific-charge.

For the sake of simplicity, we assume that $U=0$. After the double integration of equation (3.4.4) over $t$ from time 
moment of the reduce beginning, we find, that $H_{2}=H_{\mathrm{m}}+\tau g\left(U_{\mathrm{H}} / g_{0}-t\right)$. Formula (3.4.1) and the expressions for $H_{1}^{2}, \stackrel{\mathrm{m}}{H}_{2}, Q_{1}$, and $Q_{2}$, describe the electric field for the excavation explosion at the different stage of the process. When $t=U_{H} g_{0}^{-1}\left(1+\sqrt{g_{0} / g}\right)$ the first term in (3.4.1) is equal to zero, and when $t_{*}=U_{H} g_{0}^{-1}+H_{m}(g \tau)^{-1}$ (the full setting of the dust particles) the field disappears, $E=0$. So, the measurements of the setting time $t_{*}$ allows to estimate (for lack of the wind) the average size of the dust particles.

The results of the calculations according to the received formulas, which are compared with the experimental dependence, are shown at Figure 3.4 .11 (curve 2). The next parameters were used for these calculations: $\eta=1.7 \cdot 10^{-5} \mathrm{~Pa} \cdot \mathrm{s}, \quad a=48 \mathrm{\mu m}$. Beside that, we used the kinematic characteristics of the dome's development, which were received from the filming of the explosion development: $g_{0}=23 \mathrm{~m} / \mathrm{s}^{2}, U_{H}=14 \mathrm{~m} / \mathrm{s}$. Parameters $Q_{0}=1.84 \mu \mathrm{C}$ and $H_{m}=4.2 \mathrm{~m}$ were derived from a least-squares fit to the experimental data. It should be noted, that the shape of the initial spike considerably depends on the distribution of the charges in the dome. The signals becomes smooth only at a great distance $R$, where the dipole approximation is true. Sharp peak of the overshoot shape is consequence of the approximation about the simultaneous setting of the soil particles.

3.4.4 Influence of the explosive charge mass on the electric field disturbances.

The values of the effective electric charges $q^{-}$and $q^{+}$, created in the epicentral area of the underground explosion, were calculated on the base of the results of $E_{z}(t, R)$ with use of the dependence $E_{z}(R)$, described by (3.4.2). To calculate qand $q^{+}$the experimental values of $E_{2}$ and $E_{z}^{+}$were used correspondingly. The dependence of $q^{-}$on the explosion depth is represented at Figure 3.4.12 for three values of the explosive mass, used in the experiments.

From this figure we can see, that the receive dependencies have a characteristic maximum, which approximately corresponds to the same depth $W=W_{0} \quad\left(W_{0} / C^{1 / 3}=0.2 \div 0.3 \mathrm{~m} / \mathrm{kg}^{1 / 3}\right)$ of burst. The type of the dependence $q^{+}(W)$ is analogous to the 


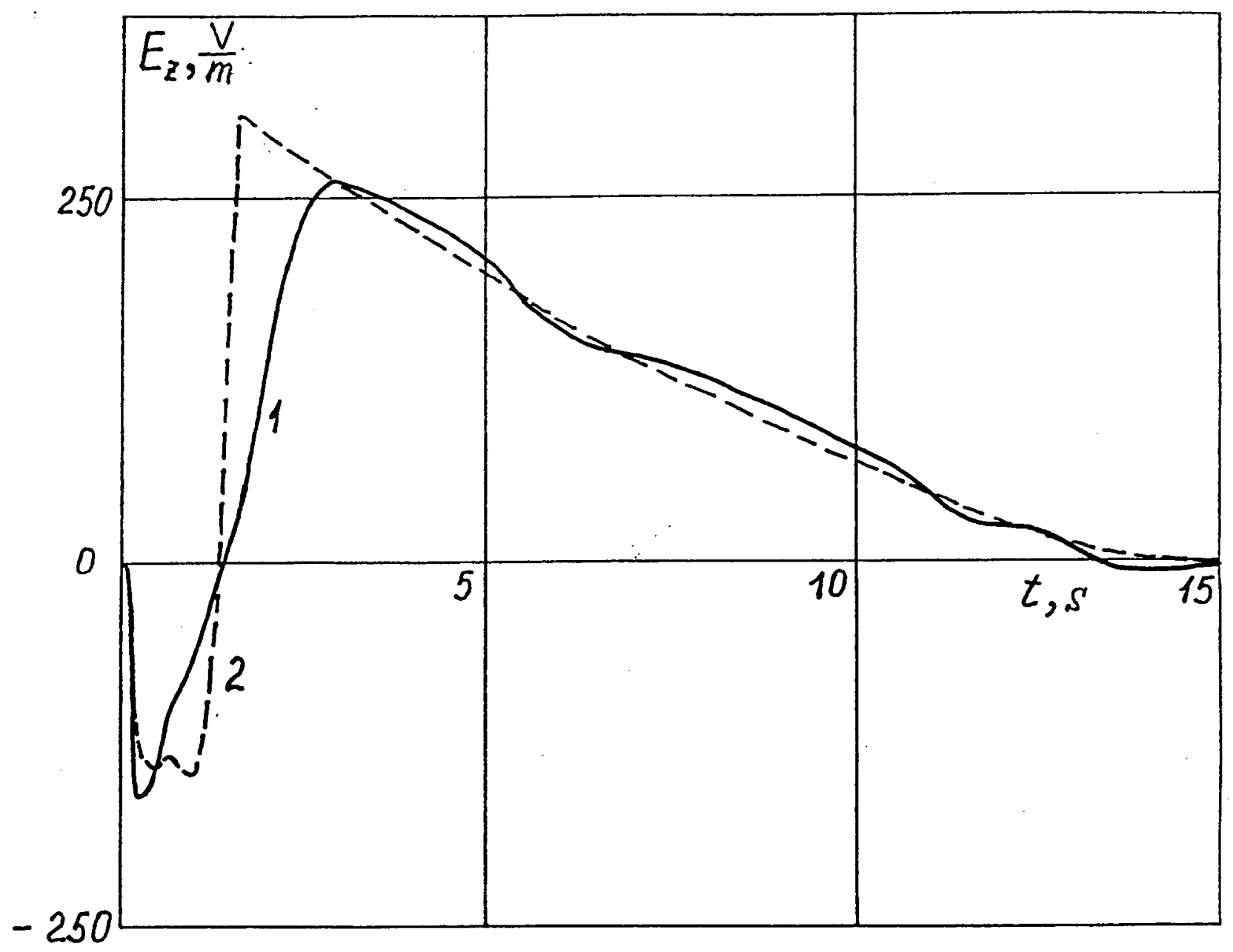

Figure 3.4.11. $E(t)$ generated by explosion in sundy pool. $C=0.023 \mathrm{~kg}, R=5 \mathrm{~m}$. 1- recorded field; 2- evaluated field. 
dependence, shown at Figure 3.4.12.

Analysis of the calculation of the effective electric charge in dependence on the main parameters of the explosive charge location, its mass, and the depth of burst showed, that with the fixed depth $W_{\text {o of }}$ ourst the magnitude of $q$ depends on the explosive charge mass as

$$
q=k \cdot C^{0.5 \pm 0.085},
$$

where $C$ is measured in $\mathrm{kg}$, and $q$ is measured in Coulomb. This dependence for the value of the maximum effective electric charge, formed after the explosions at depth of $W / C^{1 / 3}=0.2 \div 0.3 \mathrm{~m} / \mathrm{kg}^{1 / 3}$ is represented at Figure 3.4 .13 . Besides the dependence (3.4.5) there are the values of the effective electric charges, calculated on the base of the measurements of $E_{z}(t)$ during the large-scale explosion of the charge with mass $2.6 \mathrm{kT}$ (Middle Asia, 1983), at this figure. From Figure 3.4 .13 it follows, that $k=2 \cdot 10^{-4} \mathrm{c} / \mathrm{kg}^{1 / 2}$ in this case of the formation of the maximum effective electric charge. It should be noted, that this coefficient in formula (3.4.5) determines the efficiency of the underground explosion with respect to the formation of the electric charge in the atmosphere per unit of the explosive mass.

Using the expression (3.4.5) we can represent the $W / C^{1 / 3}$ dependence of the magnitude of the effective electric charge $q^{-}$ for the different masses of the explosive charge (see Figure 3.4.12) as a unified $W / C^{1 / 3}$ dependence of $q / C^{1 / 2}$. This dependence is shown at Figure 3.4.14.

Let's estimate the sources energy $U$ from the results of the calculation of the effective electric charge for the different masses of the explosive charge: $U=q^{2} / 8 \pi \varepsilon_{0} H$. The energy $U$, divided by the explosive charge energy, will give the coefficient of the transformation of the explosive energy to the energy of the electric field $\eta=U / C$. The coefficient $\eta$ for different masses of the explosive charge for explosions at depth $W / C^{1 / 3}=0.2 \div 0.3 \mathrm{~m} / \mathrm{kg}^{1 / 3}$ are represented in Table 3.4 .2 .

We can see, that only $0.0001 \%$ from the total explosion power is used to the formation of the electric field in the atmosphere.

Adushkin et al. [1990] considered a possible mechanism of electric field generation by underground bursț. This mechanism 


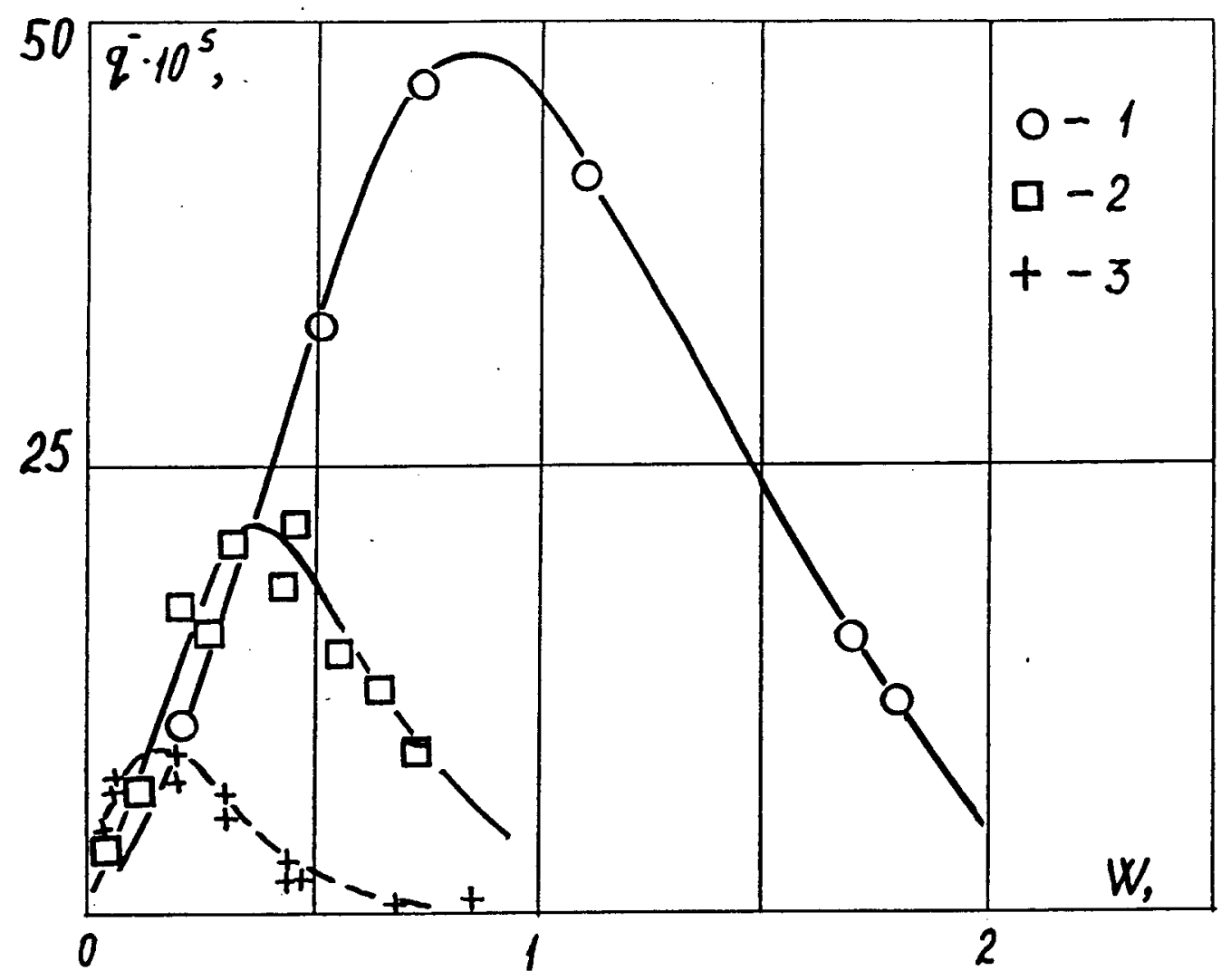

Figure 3.4.12. Magnitude of the effective eleotrio charge $q^{-}$ v.s. depth of burst $W$. $1-C=12.8 \mathrm{~kg} ; 2-C=1.6 \mathrm{~kg} ; 3-C=0.4 \mathrm{~kg}$. 


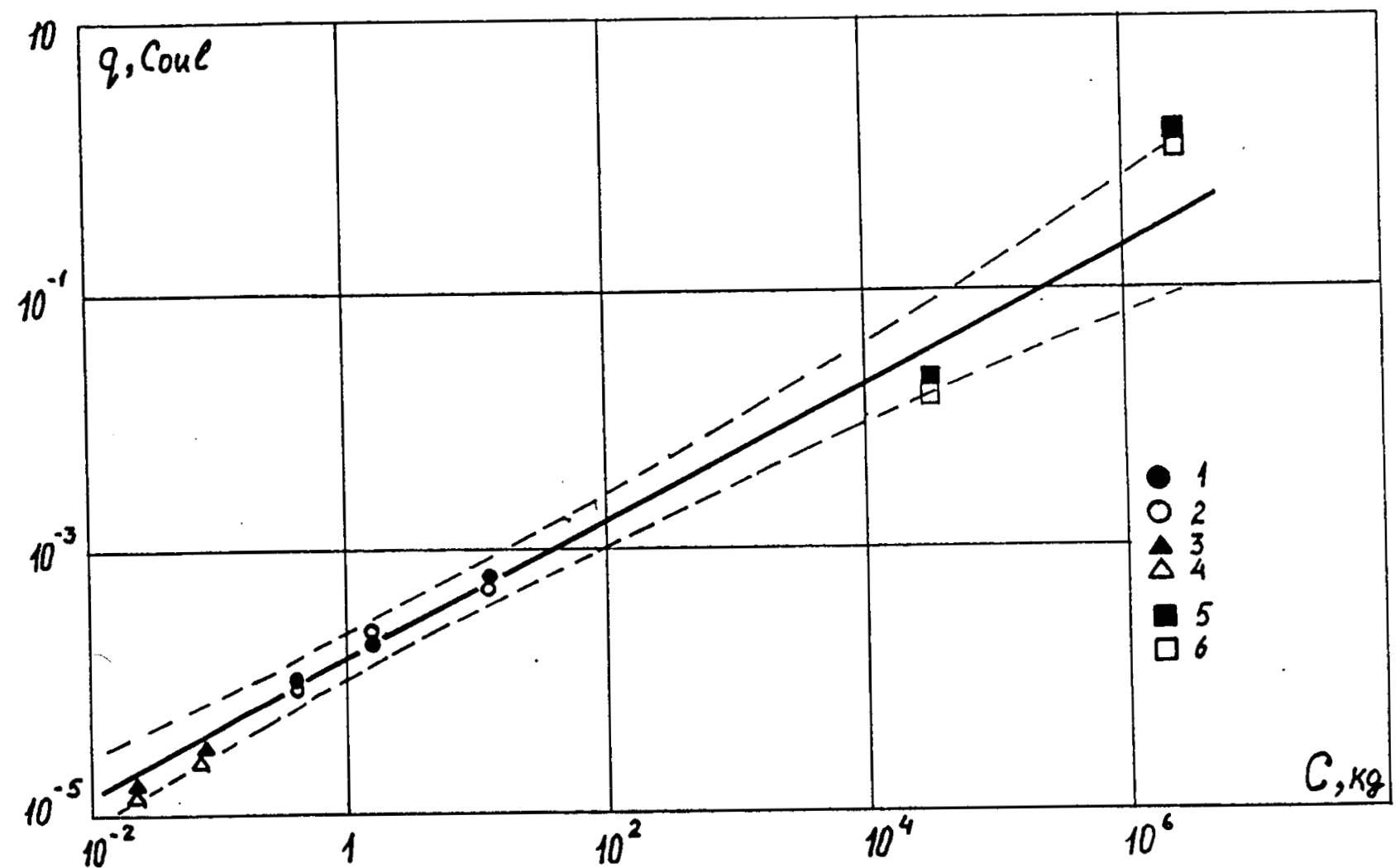

Figure 3.4.13. Relation between the magnitude of the effective electric charge and the mass of the high explosive charge for the depth of burst $W_{0} / C^{1 / 3}=0.2+0.3 \mathrm{~m} / \mathrm{kg}^{1 / 3}$. solid- a best itt line for $C=0.4 \mathrm{~kg}, C=1.6 \mathrm{~kg}$, $C=12.8 \mathrm{~kg} .(1,3,5)-q^{+} ;(2,4,6)-q^{-}$. 


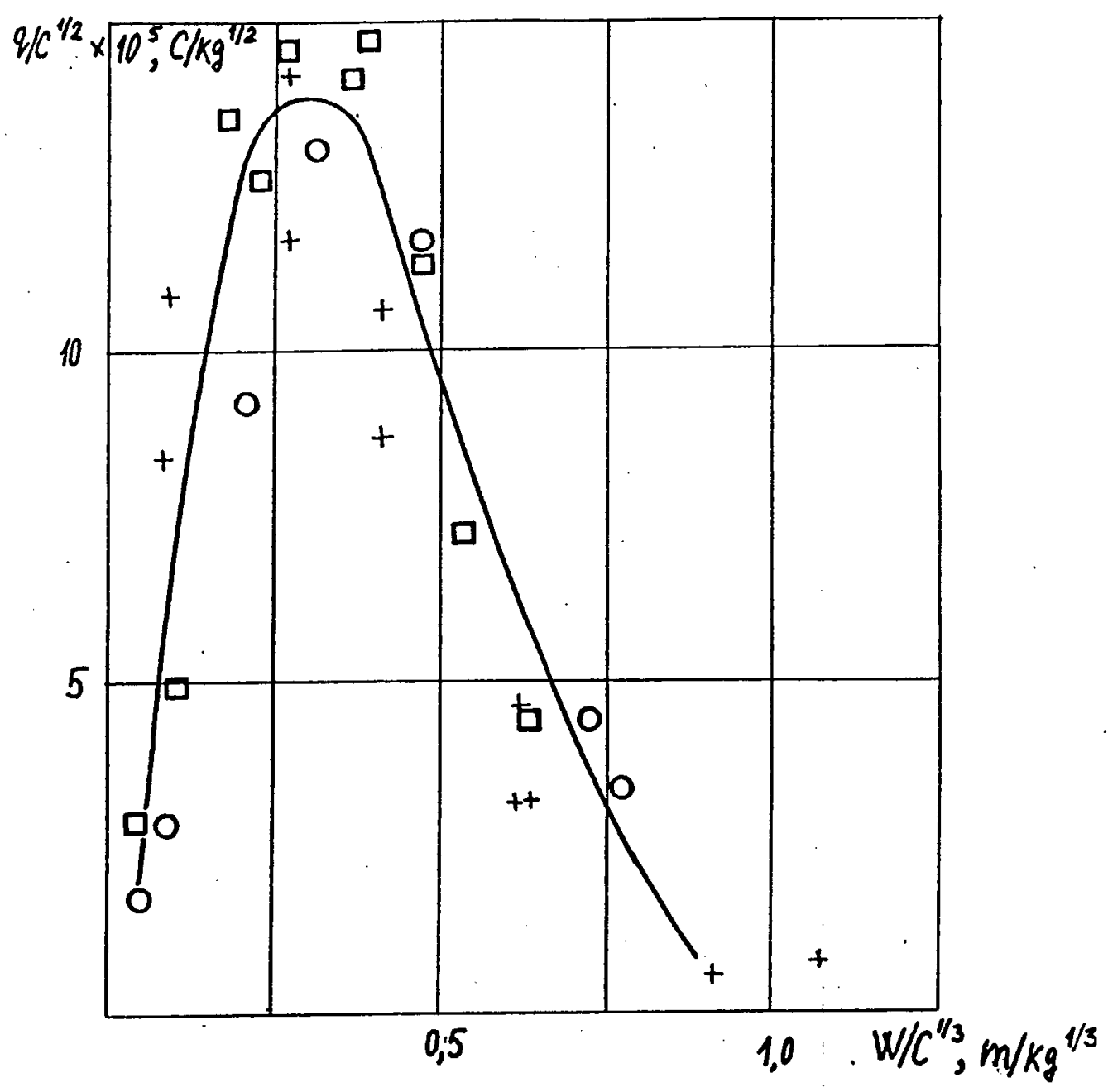

Figure 3.4.14. Magnitude of the effective electric charge $q^{-} / C^{1 / 2}$ v.s. depth of burst $W / C^{1 / 3}$. $1-C=12.8 \mathrm{~kg} ; 2-C=1.6 \mathrm{~kg} ; 3-C=0.4 \mathrm{~kg}$. 
Table 3.4.2.

\begin{tabular}{|c|c|c|c|c|c|}
\hline c, кए & 0.023 & 0.4 & 1.6 & 12.8 & $2.6 \cdot 10^{6}$ \\
\hline$\eta \cdot 10^{6}$ & 2.1 & 3.5 & 4.2 & 1.4 & 3.4 \\
\hline
\end{tabular}

is connected with the electrification of soil particles and the separation of electric charges between the ionized cavity gas and the soil particles during filtration of the ionized cavity gas through the fragmented soil. Equations obtained by Adushkin et al. [1990], permit the dependence between $q$ and $C$ to be found for two types of filtration flow: $q$ proportional $C^{1 / 2}$ for laminar filtration and $q$ proportional $C^{5 / 9}$ for turbulent filtration. This dependence correlates with the empirical equation (3.4.5).

3.4.5 Electric field generated by the underground nuclear explosions.

The experimental study of the disturbances of the electric field in the surface layer of the atmosphere were carried out during the underground nuclear explosions in the tunnel and in the borehole (Semipalatinsk Test Site and Novaya Zemlya Test Site, see Table 3.1).

The disturbance of the vertical component of the potential gradient $E$ of the electric field was measured in the air by a "field mill" which senses the atmospheric electric field. This instrument was installed on the ground surface at a distance $R=1+10 \mathrm{~km}$ from the epicentre.

Figure 3.4.15-17 represents typical records of $E_{z}(t)$ that was measured at distances $R$ from the epicentre.

Intensive deformations and destructions of the medium by the expanded products of the explosion take place in the vicinity of the underground chamber (only contained explosions are considered here). Intensive crack formation of the free surface and lifting of a spall layer of the soil are observed after the output of the compression wave on this surface. In result, the dust cloud, which is the space electrical charge, 


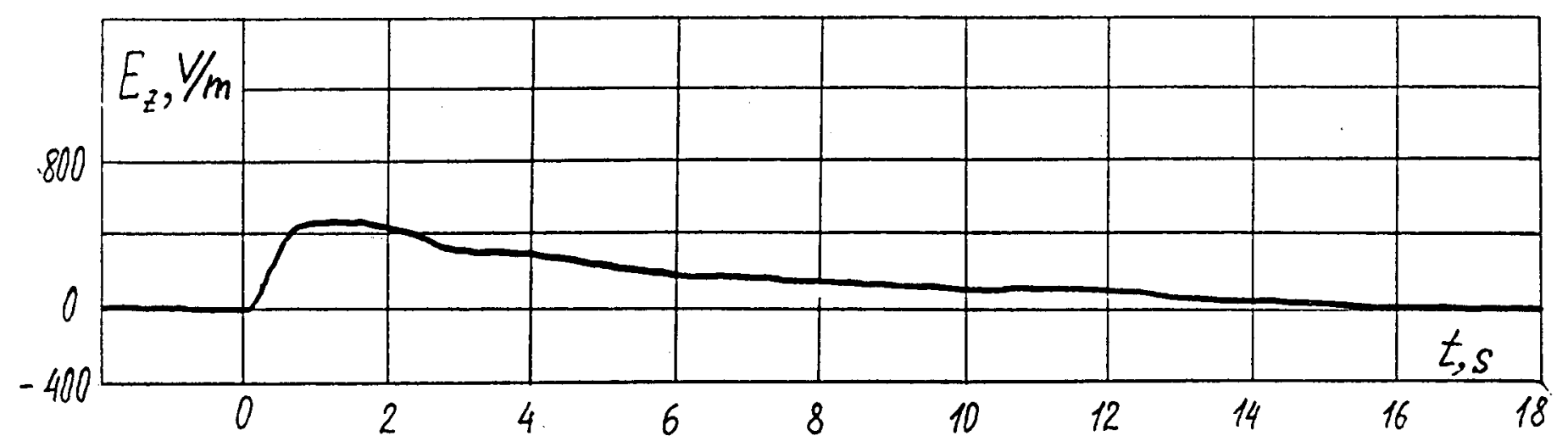

Figure 3.4.15. Electric field generated by the underground nuclear explosion. Test 215; $R=1500 \mathrm{~m}$. 


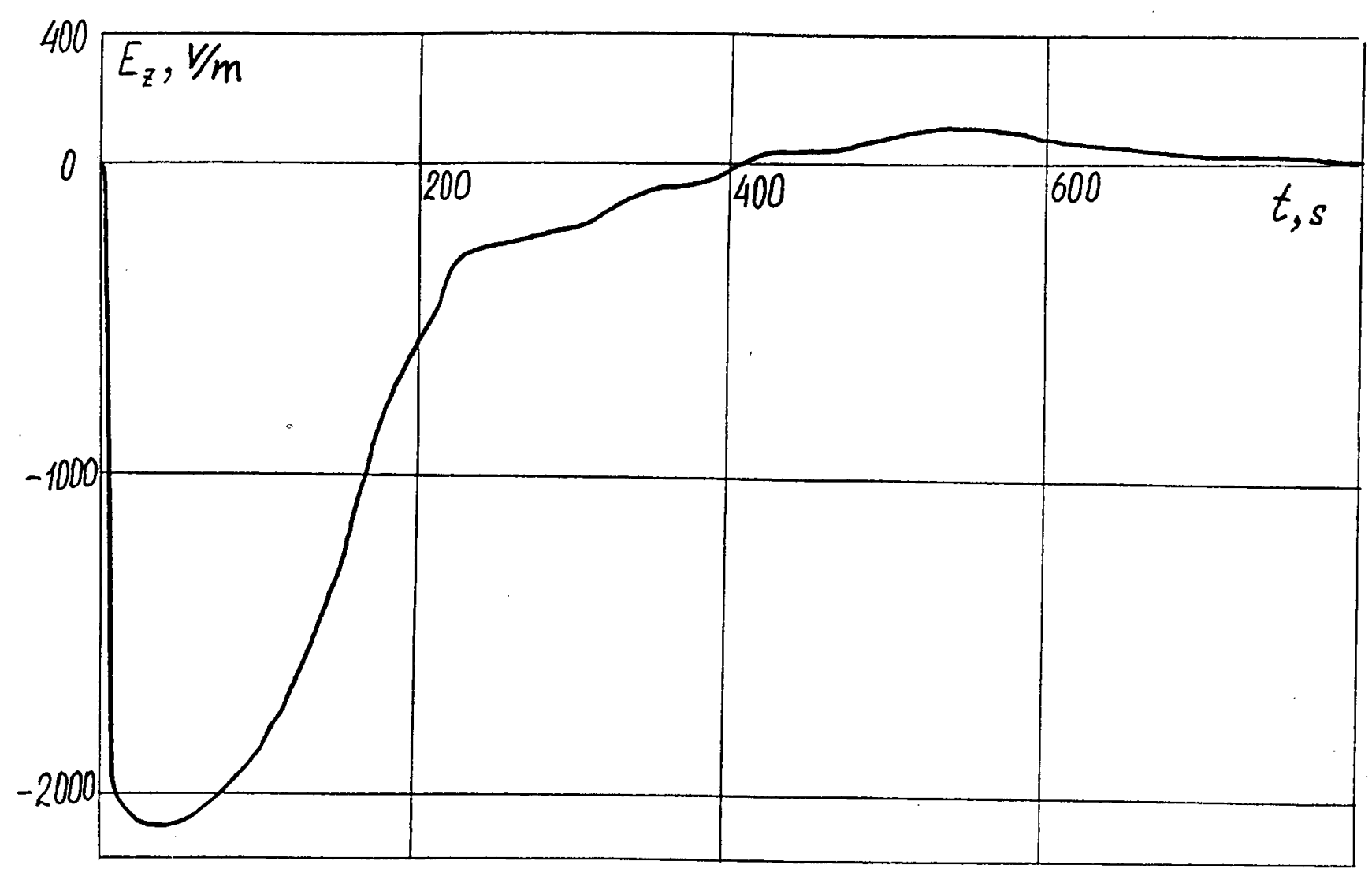

Figure 3.4.16. Electric field generated by the underground nuclear explosion. Test 1316; $R=1600 \mathrm{~m}$. 


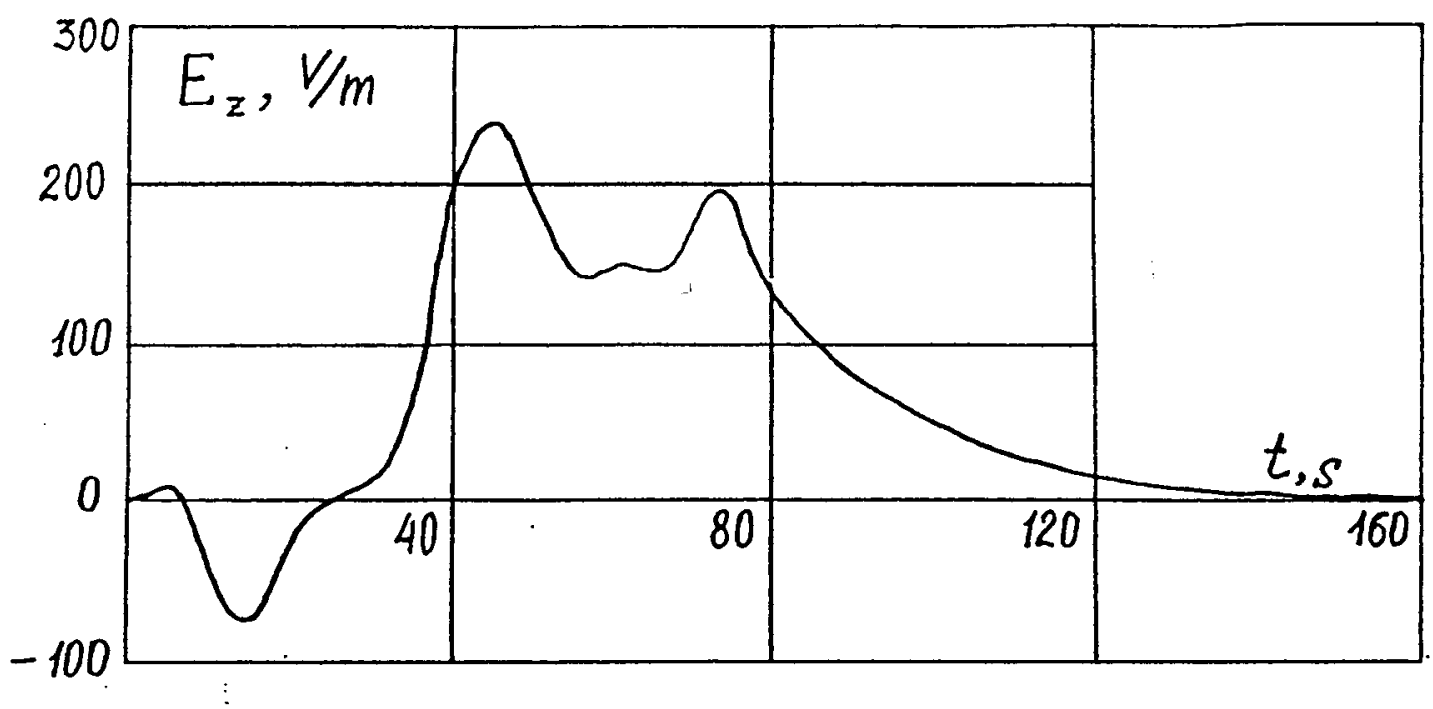

Figure 3.4.17. Electric field generated by the underground nuclear explosion. Test A-18; $R=1500 \mathrm{~m}$. 


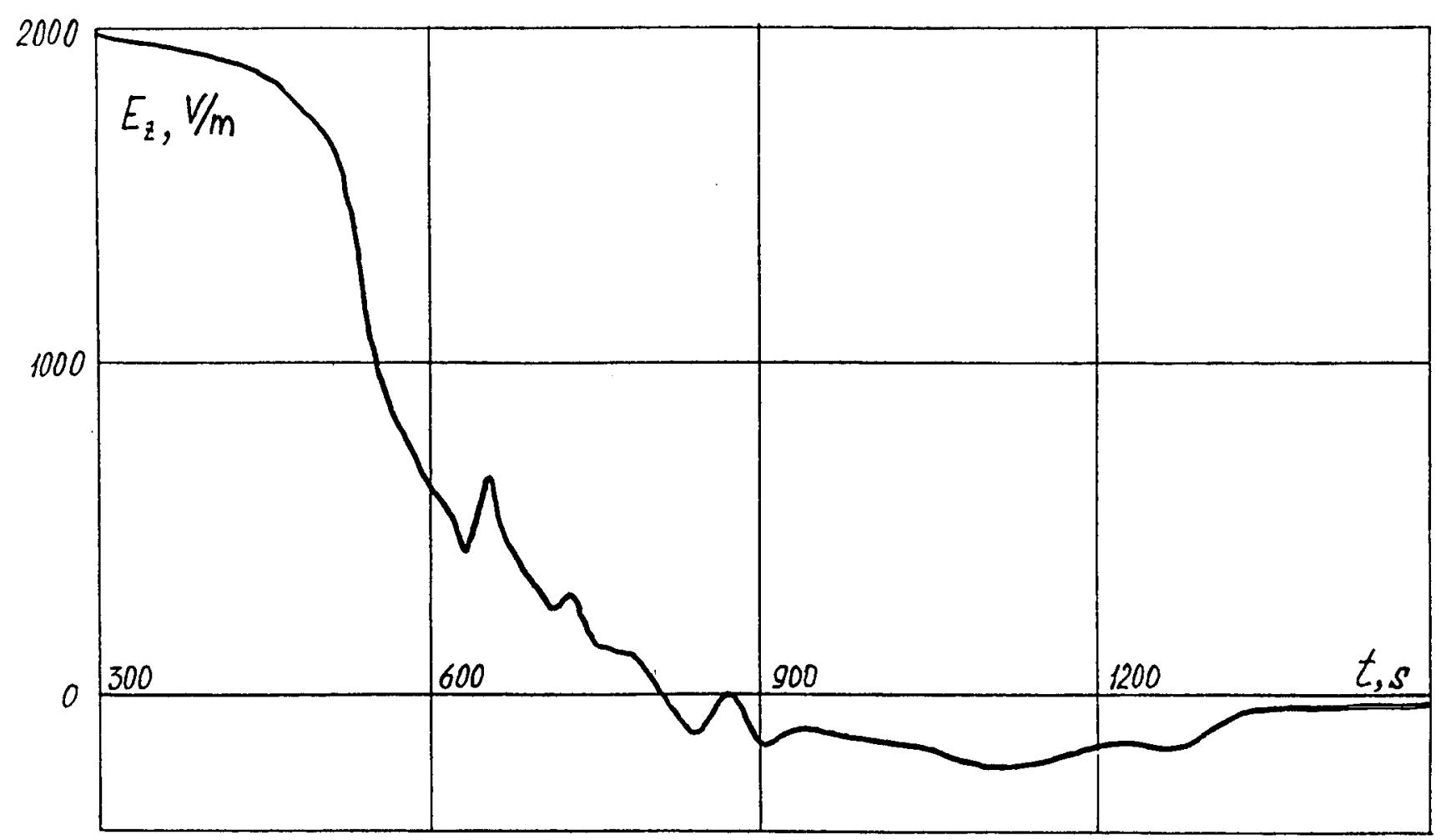

Figure 3.4.18. Eleotric field generated by the underground nuclear explosion. Test A-37; $R=1500 \mathrm{~m}$. 
generates in the surface layer of the atmosphere.

In section 3.4 .2 we considered the influence of the soil properties on the electric field disturbances. The shape of the registered signals $E_{z}(t)$, represented at Figure 3.4 .15 and Figure 3.4.16, for borehole differs from test in tunnel. The explosion in the borehole is characterized by the fact, that the surface layer of the soil from 10 to 30 meters is the sandy loam in which the small particles are contented. From the other side the tunnel explosion was carried out in the rock without this cover. Experimental explosions in the sandy loam with moisture up to 5\% of the high explosive charges with mass up to $12 \mathrm{~kg}$ showed that there is the tendency to keep the negative polarity of the signals $E_{\mathrm{z}}(t)$ in the nearest zone of the explosion.

The electric field signals, registered at different distances from the explosion epicentre (Novaya Zemlya Test Site), are shown at Figure 3.4.17.

Output of the radioactive products to the atmosphere make a considerable contribution to the disturbances of the atmospheric electric field due to the underground nuclear explosion. Radioactive cloud from the explosion products during its motion is a generator of the space electric charge in the atmosphere. The record of $E_{z}(t)$ for the tunnel explosion at Novaya Zemlya Test Site (Figure 3.4.18) is example of the disturbances, connected with the motion of the radioactive cloud of the explosion products. The "field mill" was installed at the foot of a mountain. 
4.Iong-term anomalies of the earth's electromagnetic field in the vicinity of underground explosions.

4.1. Effects of the shock magnetization of heterogeneous media.

A shock influence of non-uniform magnetic media often results in the rise of the quasistatic magnetic field, which is bound up with irreversible magnetization of the matter by the action of the shock [Kondorsky, 1940, 1959, Goodenough, 1954, Polivanov, 1957]. Explosions and shock waves in the geological media create residual magnetic fields too. These magnetic fields are especially noticeable in the epicentral zone of the explosion [Stacey, 1964, Undzenkov and Shapiro, 1967, Barsukov and Skovorodkin, 1969, Shapiro and Ivanov, 1969]. The irreversible magnetization forms for a few seconds, whereas the relaxation lasts for more than twenty-four hours [Hasbrouk and Allen, 1972, Akopijn et al., 1973, Kozlov et al., 1985]. This effect is connected with the magnetization of ferromagnetic fragments, included in the natural rocks with small concentrations.

In this section we'll investigate effects of the shock magnetization of non-uniform medium by underground explosions and shock influences. Besides that, we'll consider effects, conditioned by the magnetization of a casing and some other metallic objects in the zone of the influence of the mechanical stresses wave.

It was experimentally. [Stacey, 1964, Undzenkov and Shapiro, 1967, Barsukov and Skovorodkin, 1969, Shapiro and Ivanov, 1969] and theoretically [Kondorsky, 1959] shown, that the irreversible magnetization of the matter, arising after the shock, is proportional to the mechanical stress and it is directed along the extermal magnetic field. Let's consider uniform medium with initial magnetization $J=c o n s t$ directed as a local geomagnetic field. Let the shock wave arise under the influence of an explosion or some other source, and this wave result in the irreversible change of the medium magnetization.

At first, let's consider the case of the spherically symmetrical disturbance of the stresses near the shock wave source. In the destruction zone with radius $R_{0}$, the matter magnetization, because of the re-packing of the rock pieces, 
has a chaotic character. So, we can neglect the contribution of this zone in the magnetic field. In the zone of elastic deformations with radius $R$, where $R$ is the distance to the elastic wave front, the magnetization increment is determined as $\Delta J=\vec{J} \cdot \sigma_{r r} / A$, where $A \approx 1 \mathrm{GPa}$ is an experimental parameter [Stacey, 1964 ], and $\sigma_{r r}=\sigma_{r r}(r)$ is a maximum absolute value of the radial stress at a given point of the medium with radius $r$.

Let's integrate magnetic fields of the elementary magnetic moments $d J$ for the spherical symmetry of the stresses. We'II receive the induction of the magnetic field $B$ at arbitrary point $r$ :

$$
\vec{B}=-\nabla \frac{(\vec{d} \vec{m})}{r^{3}}, \quad \quad \vec{d}_{\mathrm{m}}=\frac{\mu_{0} \vec{J}}{A} \int_{R_{0}}^{r} r^{\prime 2} \sigma_{r r}\left(r^{\prime}\right) d r, \quad r<R .
$$

If $r \geq R$, the upper limit of the integral (4.1) is equal to $R$. At great distance away, where the stresses don't influence on the medium magnetization, the magnetic field, as it was to be expected, has a dipole character. This type of the dependence has been proposed by Kozlov et al. [1974]. But the experimental investigations [Erjanov et al., 1985] at distances of $0.5-10 \mathrm{~km}$ from the explosion centre showed, that the character of the residual magnetic field decrease is another: $B \sim r^{-1}$.

To analyze this appropriateness let's "appeal to the formula (4.1), taking into account the fact, that if $r<R$ the amplitude of the stresses in the elastic zone changes in accordance with the law $\sigma_{\mathrm{rr}}=P_{*} \cdot R_{0} / r$, where $P_{*}$ is the tensile strength of the rock. So, from the equation (4.1) we can receive the next relation for the zone of $R_{0}<r<R$ :

$$
\vec{B}=\frac{\mu_{0} P_{*} R_{0}}{2 A r}\left(1-\frac{R_{0}^{2}}{r^{2}}\right) \cdot\left(\frac{3(\vec{J} \overrightarrow{\vec{J}}) \vec{r}}{r^{2}}-\vec{J}\right)
$$

If the elastic wave passed far enough, the magnetic field at great distances from the source $\left(R_{0} \ll r\right)$, as is evident from (4.2), decreases with the distance, and this decrease is much slower than the dipole decrease, because $B \sim r^{-1}$.

Figure 4.1 shows the experimental dependence $B(r)$ (curve 1), founded by Erjanov et al. [1985], after the surface chemical explosion with mass of 251 tons (MASSA experiment). 


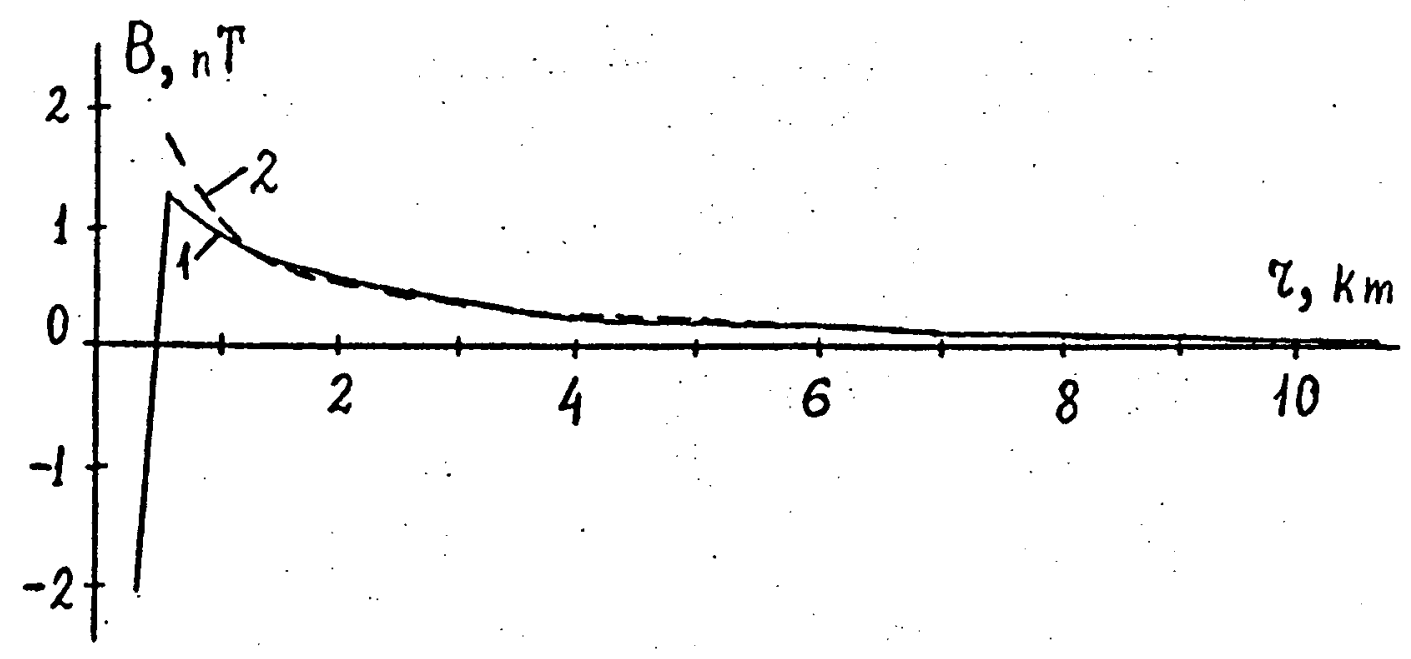

Figure 4.1. Magnetic field $B$ v.s. distance $r$.

1- experimental dependence $B(r)$ [Erjanov et al., 1985];

2- theoretical dependence. 
Results of the calculations according to the formula (4.2) with the next parameters: $P_{*}=0.1 \mathrm{GPa}, A=1 \mathrm{GPa}, R_{0}=100 \mathrm{~m}$, $J=0.12 \mathrm{~A} / \mathrm{m}$, an angle of the slope of the vector $J$ to the Earth's surface was chosen as $\pi / 4$, are represented by the dotted Iine 2 at this iigure. The difference between these dependencies when $r<0.5 \mathrm{~km}$ might be conditioned by the influence of other mechanisms of the static magnetic field formation, which will be considered bellow.

Spherical symmetry of the task disappears after the reflection of the elastic wave from the surface. It may result in the increase of the magnetic field, as it will be show below. In order to estimate the influence of the free surface let's consider the source of the mechanical stresses at the boundary of the elastic half-space $y_{\rightarrow}<0$ (Figure 4.2). Vector of the initial medium magnetization $J$ lies on the plane, which contains $z$-axis and forms an angle $\gamma$ with the medium surface.

In this case it is convenient to carry out the integration by the volume in the spherical coordinates with a polar angle $\theta$, measured from z-axis. So, for the projection of the magnetic field on $z$-axis we have:

$$
\begin{gathered}
B_{z}(0,0, z)=\frac{\mu_{0} J}{4 \pi A} \int_{R_{0}}^{R} r^{\prime 2} d r \int_{0}^{\pi} \frac{\sin \theta}{\rho^{\prime 3}} d \theta \int_{\gamma}^{\pi_{+} \gamma} \frac{3\left(z-r^{\prime} \cos \theta\right)}{\rho^{\prime 2}}\left(\cos \Psi\left(z-r^{\prime} \cos \theta\right)-\right. \\
\left.\left.-r^{\prime} \cos \Phi \sin \Psi \sin \theta\right)-\cos \Psi\right) \sigma_{\mathrm{r} r}\left(r^{\prime}, \theta, \Phi\right) d \Phi ; \\
\rho=\left(z^{2}+r^{\prime 2}-2 z r^{\prime} \cos \theta\right)^{1 / 2},
\end{gathered}
$$

where $\Phi$ is an azimuth angle, and $\Psi$ is an angle between the vector $J$ and $z$-axis. For simplicity assume that $\sigma_{r r}=\sigma_{r r}(r)$. After the integration in the equation (4.3) by $\Phi$ and $\Psi$ we'II receive:

$$
\begin{gathered}
B_{z}(0,0, z)=\frac{\mu_{0} J}{\pi A z^{3}}\left\{\int_{R_{0}}^{z}\left(\pi r^{\prime} \cos \Psi-\sin \Psi \sin \gamma \cdot z \frac{d}{d r^{\prime}}\left(r^{\prime} f\left(\frac{r^{\prime}}{z}\right)\right)\right] r^{\prime} \sigma_{r \Gamma}\left(r^{\prime}\right) d r^{\prime}+\right. \\
+\sin \Psi \sin \gamma \int_{z}^{R} \frac{d}{d r^{\prime}}\left(r^{\prime} f\left(\frac{z}{r^{\prime}}\right)\right) r^{\prime} \sigma_{r r}\left(r^{\prime}\right) d r^{\prime} ; \\
f(x)=K(x)-E(x)
\end{gathered}
$$

where $K(x)$ and $E(x)$ are full elliptical integrals of the first 


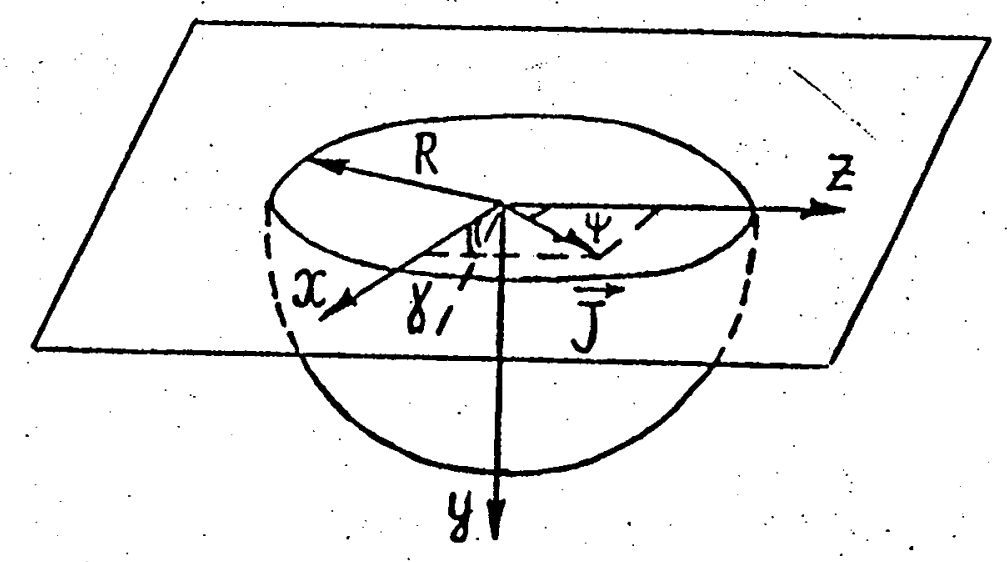

Figure 4.2. Coordinates and orientation of the vector of the initial medium magnetization $\vec{J}$. 
and second, kind. If $z<R$, the second integral in the expression (4.4) disappears, and the upper limit of the integration in the first integral is $R$.

If the law $\sigma_{r r}{ }^{2} r^{-1}$ is correct, from the equation (4.4) we can receive the next expression in the range of $R_{0} \ll z \ll R$ :

$$
B_{z}(0,0, z)=\frac{\mu_{0} J P_{*} R_{0}}{2 A z}\left[\cos \Psi+\frac{R \sin \Psi \sin \gamma}{2 Z}\right] .
$$

As is obvious from this relation, the estimation (4.2) increases in $R / Z$ times. While the wave travels a distance of $R$ 10-20 km, the field increases up to the value of $B$, $20 \mathrm{nT}$. In fact, the formula (4.5) gives only rough estimation of the effect, because there isn't detail consideration of the complex distribution of the stresses near the surface. So, unlimited growth of $B$ when $R \rightarrow \infty$ is connected with the fact, that the influence of the free surface on the stresses wasn't taken into account in the equation (4.5), whereas, in average, $\sigma_{\mathrm{rr}}$ decreases with the distance faster than $r^{-1}$.

As to underground explosions, it's necessary to take into account the shock influence on the technical structures and constructions, made from magnetic materials. For example, the presence of the casing and some other massive metallic objects, which are magnetizable in the Earth's magnetic field after the shock, may result in the formation of static magnetic fields.

In order to estimate this effect let's consider the magnetic field, conditioned by the effect of the shock magnetization of the casing. We can imagine casing as an uniformly magnetized vertical bar with length of $l$ and radius $r_{0} \ll l$. If the distance to the bar $\rho$ (a polar radius is in range of $r$ 。 $\rho \ll l$, than the field far from its ends is determined as:

$$
\vec{B}=\frac{\mu_{0} r_{0}^{2}}{2 \rho^{2}}\left(\frac{2 \vec{\rho}\left(\vec{J}_{\tau} \vec{\rho}\right)}{\rho^{2}}-\vec{J}_{\tau}\right),
$$

where $\vec{J}_{\tau}$ is the component of the magnetization vector, perpendicular to the bar. In accordance with the conclusions of Kondorsky [1959], it is possible to achieve the magnetization of $J_{\sim} 0: 1 J_{s}$, where $J_{s}$ corresponds to the satiety state, by the repeated shaking of the bar in the Earth's magnetic field. For the iron-bar $\left(J_{s} 2 \cdot 10^{6} \mathrm{~A} / \mathrm{m}\right)$ with radius $r_{0}=0.2 \mathrm{~m}$ from 
the equation (4.6) $B$ is about $20 \mathrm{nT}$ at a distance of $\rho$. $500 \mathrm{~m}$.

At a great distance $(\rho \gg \tau)$ the bar field is determined by the formula (4.1), where $4 \pi \alpha_{m} / \mu_{0}$ is a magnetic moment of the bar. So, assuming that $l=100 \mathrm{~m}$ and $\rho=1 \mathrm{~km}$, we receive $B$. $0.3 \mathrm{nT}$, that corresponds to the experimental values.

These estimations show, that near the explosion centre the static magnetic fields might be connected with the magnetization of the rock as well as of the metallic constructions. But, at great distances the first effect is more significant because its value decreases with the distance slower.

4.2. Effects of the currents redistribution and change of the conductivity in fragmented media.

In this section we'll estimate the influence of the shock action on the magnetic fields, which are result of the natural Earth's currents and currents with artificial origin, conditioned by electro-chemical processes on the surface of the casing.

An appearance of the contact potential difference on the surface of the metallic tube is connected with the difference of the conductivity types: an electronic conductivity in the metal, and an ionic - in the medium around the tube. Analogous effect is known for are deposits [Semenov, 1974]. Contact electromotive force depends on the depth, because the mineral composition of the water, consisted in the rock, changes with the depth. In result, currents, which distort local electromagnetic field, arise in the casing and in medium around it. This field has a static character, and might be measured before the explosion realization.

The shock influence of the explosion results in the change of the conductivity in the fragmented volume of the rock. Redistribution of the currents in ones turm results in the additional disturbance of the field, which has an irreversible character.

To estimate the mentioned effect let's consider a thin metallic bar with length $l$, dipped in the homogeneous medium 
with conductivity $\sigma$ (Figure 4.3). Let's suppose, that the upper part of the bar is a negative electrode, whereas the lower part is a positive electrode, as it is usual for ore metallic bodies [Semenov, 1974]. The explosion action results in the formation of the spherical area of the fragmented rock with radius $R_{0}$ in the medium. The conductivity in this area is $\sigma_{p}$.

We assume, that the currents distribution is stationary and total current $I$ is known. The potential $\varphi$ of the electric field is determined by the Poisson's equation with regard for the fact, that $\varphi$ and its derivative by radius are continuous on the sphere boundary, and the normal component of the current density becomes equal to zero on the medium free surface. Solution of this task for the point electrode without regard for the free surface is:

$$
\begin{array}{ccc}
\delta \varphi=\frac{\delta I}{4 \pi \sigma}\left[-\frac{1}{R}+\left(\sigma-\sigma_{p}\right) \sum_{n=1}^{\infty} \frac{n \lambda_{n} P_{n}(\cos \theta)}{n \sigma_{p}+(n+1) \sigma}\right], & r_{0}>R ; \\
\lambda_{n}=\frac{r_{0}^{n}}{r^{n+1}}, \quad r_{0}>R ; \quad \lambda_{n}=\frac{R_{0}^{2 n+1}}{\left(r_{0} r\right)^{n+1}}, \quad r_{0}>R ;
\end{array}
$$

where $\delta \varphi$ and $\delta I$ are the potential and the current of the point electrode, correspondingly, $r_{0}$ is the distance from electrode to the sphere centre, and $P_{n}(\cos \theta)$ are the Legendre polynomials. Values of $R, r$ and $\theta$ are shown at Figure 4.3. The first item of the equation (4.7) describes non-disturbing solution, corresponding to the case of $\sigma=\sigma_{p}$.

Let the currents, generated by the bar parts, are:

$$
\delta I_{-}=-I \frac{d r_{0}}{l_{0}}, \quad \delta I_{+}=I \frac{d r_{0}}{l-l_{0}},
$$

where $l_{0}$ is the length of the negative electrode. Let's substitute the expression (4.8) into (4.7) and integrate this equation by $r$, than we'll receive the solution of the task for the electrode with finite length in the unlimited medium. Adding the potential, created by the mirror source to the solution, we can roughly take into account the influence of the free surface $z=0$ : 


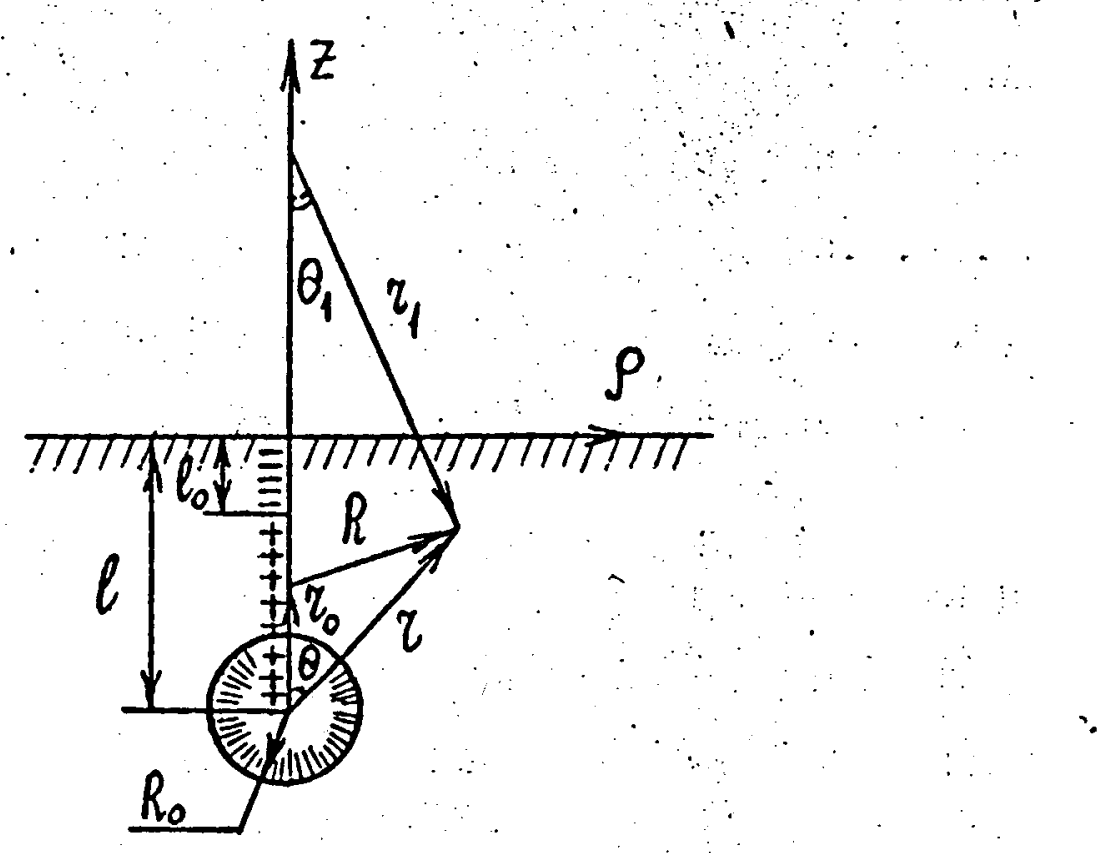

Figure 4.3. Schematic distribution of the contact potential difference on the surface of the metallic tube. $R_{0}$ - radius of the spherical area of the fragmented rock. 


$$
\begin{aligned}
& \varphi=\varphi_{0}(\rho, z)+\varphi_{0}(\rho,-z)+\varphi_{\mathrm{i}}(r, \theta)+\varphi_{\mathrm{i}}\left(r_{1}, \theta_{1}\right), \quad z \leq 0 ; \\
& \varphi_{0}=\frac{I}{4 \pi \sigma\left(l-l_{0}\right)}\left[\ln \frac{\left(\left(z+l_{0}\right)^{2}+\rho^{2}\right)^{1 / 2}-z-l_{0}}{\left((z+l)^{2}+\rho^{2}\right)^{1 / 2}-z-l}+\right. \\
& \left.\quad+\left(1-\frac{l_{l}}{l_{0}}\right) \ln \frac{\left(\left(z+l_{0}\right)^{2}+\rho^{2}\right)^{1 / 2}+z+l_{0}}{\left(z^{2}+\rho^{2}\right)^{1 / 2}+z}\right], \\
& \varphi_{i}=\frac{I\left(\sigma-\sigma_{p}\right)}{4 \pi \sigma\left(l-l_{0}\right)} \sum_{n=1}^{\infty} \frac{\Lambda_{n} P_{n}(\cos \theta)}{n \sigma+(n+1) \sigma}\left(\frac{R_{0}}{r}\right)^{n+1}, \quad r>R_{0}, \\
& \Lambda_{n}=\frac{2 n+1}{n+1}-\frac{R_{0}^{n}}{\left(l-l_{0}\right)^{n}}-\left(\frac{l}{l_{0}}-1\right)\left[\frac{R_{0}^{n}}{\left(l-l_{0}\right)^{n}}-\frac{R_{0}^{n}}{l^{n}}\right] .
\end{aligned}
$$

where index 1 corresponds to the mirror image (Figure 4.3), and $\rho$ is a polar radius.

In this approximation we have the follow equation for the air $(z>0)$ :

$$
\varphi=\left[\varphi_{0}(\rho, z)+\varphi_{i}(r, \theta)\right]
$$

Non-disturbed solution, marked by the zero index is exact, because the conditions on the sphere boundary after the mirror reflection are correct automatically if $\sigma=\sigma$.

In the case of $R_{0} \ll \tau$ and $R_{0} \ll\left(\tau-\tau_{0}\right)$, it is possible to omit all members of the row besides the first member in the expression for $\varphi_{i}$. Moreover, if $\rho \gg l$, the member $\varphi_{0}$ becomes simpler too. So, on the plane $z=0$ we have:

$\varphi_{0}=\frac{I l\left(l-l_{0}\right)}{8 \pi \sigma \rho^{3}}, \quad \varphi_{i}=\frac{3 I\left(\sigma-\sigma_{p}\right) l R_{0}^{2}}{8 \pi\left(l-l_{0}\right) \sigma\left(\sigma_{p}+2 \sigma\right)\left(l^{2}+\rho^{2}\right)^{3 / 2}}$,

After the measuring of the experimental dependencies $\varphi(\rho)$ and their comparison with the expression (4.11), we can estimate the parameters in the equation (4.11). In particular, the values of $R_{0}$ and $\sigma$ might be useful to the estimation of the rock permeability after its destruction.

In the case of $z \gg \tau, z \gg \tau_{0}, z \gg \rho$, formulas (4.9) and (4.10) are simplified to the follow type:

$\varphi_{0}=\frac{I l}{8 \pi \sigma z^{2}}$,

$$
\varphi_{i}=\frac{3 I\left(\sigma-\sigma_{p}\right) R_{0}^{2}}{8 \pi\left(l-l_{0}\right) \sigma\left(\sigma_{p}+2 \sigma\right) z^{2}},
$$

One can see, that the field in the air decreases with the height 
slower than in the horizontal direction.

The magnetic field generated by the currents has only one azimuth component $B_{\psi}$, due to the symmetry of the task. Using equation (4.9) we can receive the next expression:

$B_{\psi}=B_{0}(z, \rho)-B_{0}(-z, \rho)+B_{i}(r, \theta)-B_{i}\left(r_{1}, \theta_{1}\right)$;

$B_{0}=\frac{\mu_{0} I}{4 \pi \rho\left(\tau-l_{0}\right)}\left(\frac{\eta}{l_{0}}\left(\left(z+l_{0}\right)^{2}+\rho^{2}\right)^{1 / 2}-\left((z+l)^{2}+\rho^{2}\right)^{1 / 2}\right)$,

$B_{i}=\frac{\mu_{0} I\left(\sigma-\sigma_{p}\right)}{4 \pi \rho\left(l-l_{0}\right)} \sin \theta \sum_{n=1}^{\infty} \frac{\Lambda_{n} p^{\prime}(\cos \theta)}{(n \sigma+(n+1) \sigma) n}\left(\frac{R_{0}}{r}\right)^{n+1}$,

where the stroke represents the derivative with respect to $\cos \theta$.

At great distances $\left(\rho \gg l, l_{0}, z\right)$ this solution is simplified to the next form:

$B_{\Psi}=B_{\Psi_{0}}+B_{\Psi_{i}} ; \quad B_{\Psi_{0}}=\frac{\mu_{0} I l\left(\tau+l_{0}\right) z}{4 \pi \rho^{4}}$

$B_{\Psi_{i}}=\frac{3 \mu_{0} I\left(\sigma-\sigma_{p}\right) R_{0}^{2} \rho}{8 \pi\left(l-l_{0}\right)\left(\sigma_{p}+2 \sigma\right)}\left(\frac{1}{\left((z-l)^{2}+\rho^{2}\right)^{3 / 2}}-\frac{1}{\left((z+l)^{2}+\rho^{2}\right)^{3 / 2}}\right)$.

Under these approximations in the air $B_{\psi}$ is equal to 0 . But under the real conditions, the field near $z=0$ has a finite value, because of the disturbance of the task symmetry and the presence of some surface roughnesses.

In contrast to the electric field $\varphi_{i}$ (4.11), which is maximum when $\rho=0$, the magnetic field $B_{\Psi_{i}}$ achieves its extreme when $\rho=\tau / 2$. Using the equations (4.11) and (4.12) it's possible to find the relation between the amplitude values of the fields at a some depth $z$ :

$$
B_{0}=\frac{\mu_{0} z \sigma \varphi_{0}}{\rho} \text {. }
$$

So, assuming that $z / \rho=0.1, \sigma=0.1 \mathrm{~s} / \mathrm{m}, B_{*}=10 \mathrm{nT}$, we'Il receive that $\varphi_{0} \sim 1 \mathrm{~V}$.

In conclusion we'll give the estimation of the shock influence on the distribution on the natural Earth's currents. Irreversible medium deformations, connected with the medium destruction and the change of the permeability of capillaries, 
result in the change of conductivity. For simplicity assume that the density of the telluric currents $f_{0}$ is constant everywhere before the destruction. Formation of the spherical zone of the destruction with conductivity $\sigma$, which differs from the conductivity of the non-destroyed matter $\sigma$, creates re-distribution of the electric potential:

$$
\varphi=-\frac{(\vec{J} \vec{r})}{\sigma}\left[1+\frac{\left(\sigma-\sigma_{p}\right) R_{o}^{3}}{\left(2 \sigma_{p}+\sigma\right) r^{3}}\right] .
$$

Electric disturbances $\delta \varphi$, conditioned by the destruction zone, are described by the second term in the square brackets. It is necessary to add the potential of the electric surface charges to the expression (4.13). In this case $\delta \varphi$ on the Earth's surface practically doubles. If $j_{0}=1 \mathrm{~mA} / \mathrm{m}^{2}, R_{0}=100 \mathrm{~m}$, $\sigma=10^{-2} \mathrm{~s} / \mathrm{m}, \quad \sigma=0, \quad r=500 \mathrm{~m}$ we can receive the next estimation: $\delta \varphi \approx 0.4 \mathrm{~V}$.

In the case of the underground explosion, a heated gaseous products which contain in the underground cavity, result in the appearance of thermoelectric and thermogalvanomagnetic effects in the environment. If there is the decay of the radioactive elements in the explosion products, that the medium heating and the temperature gradient might be supported for a long time. These processes also might be cause of the stable changes of the local electromagnetic field of the Earth near the explosion. 
Conclusions

At the next step in advancing the field we can to provide the descriptive, quantitative information on following topics:

1.Disturbances of electric field generated by detonation of high explosive on the surface of the ground

-instrumentation observations of the electric field during the large-scale (see Table 3.1) and series of small-scale chemical explosions on the surface of the ground; -dependence of the magnitude of the effective electric charge of the dust cloud on the mass of the explosive charge;

-size distribution of fine particles of the fragmented soil;

-estimation of dust concentration in the dust cloud on the basis of electric field measurement;

-disturbances of the electric field generated by the air shock wave.

2.Results of the instrumentation observation of the electric field concermed with a cavity gas venting during the underground nuclear explosions.

3. Excitation of the ionosphere during the underground nuclear and large-scale chemical explosions

-excitation of the ionosphere during nuclear tests in the atmosphere (Novay Zemlay)

-estimation of the electric field in the ionosphere due to electrical charges of the dust cloud in the surface layer of the atmosphere;

-excitation of the ionosphere by the shock wave that is propagated in vertical direction. 
References.

Abert G., Brief physical reference book, (in mussian) Moscow, Fizmatgiz, 1963.

Afinogenov I.P. et al., Devices for investigations of the atmospheric layer, nearest to the Earth, (in russian), Leningrad, Gidrometeoizdat, $1977,319 \mathrm{p}$.

Akopijn .G., Nagapetijn .V., Pushkov .N., Rassanova G.V., Skovorodkin J.P. Magnetic researches during explosions at Kadjarsky quarry region, (in mussian), News of AS Arm. SSR. The sciences about the Earth. 1973. V. 26. No. 6. P. 90-92.

Alfven G., Felthammar K.-G. Cosmic electrodynamics., (in russian), Mir, Moscow, 1967.

Alpert Ya.I. Electromagnetic waves spreading and ionosphere, (in russian), Moscow, Nauka, 1972.

Anisimov C.V., Gohberg M.B., Ivanov E.A., Pedanov M.V., Rusakov N.I., Troitskaya V.A., Goncharov V.I. Short-periodical oscillations of the Earth's electromagnetic field from the industrial explosions, (in mussian), DAN USSR, 1985, V. 281 , 16 3, pp. 556-559.

Adushkin V.V., Suhotin A.P., About the fragmentation of solid medium by explosion, (in russian), PMTF, 4, 94-101.

Adushkin V.V., Soloviev S.P., Low-frequency electric field in the surface layer of the atmosphere generated by underground burst, (in russian), DAN USSR, 299, 840-844, . 1988.

Adushkin V.V., Solovier S.P., Disturbances of the atmospheric electric field in the zone close to the epicenter of underground burst, (in russiar), IzV. Acad. Soi. USSR, Fizika Zemli, 3, 51-59, 1989.

Adushkin V.V., Soloviev S.P., Syrkov V.V., Electric field generated by the expavation explosion, (in russian), The Physics of Combation and explosions, 4, 117-121, 1990.

Adushkin V.V., Soloviev S.P., An estimate technique of the dust poliution of the atmosphere on a basis of the electric field measurement, (in mussian), The Physics and Technology of Creation of Useful minerals, 4, 88-88, 1994.

Allison F.E., Shock induced polarization in plastics. 1. Theory, J.AppI.Phys., 36, 7, 2111-2113, 1965. 
Balbachjan M.J., and Parhomenko E.I, Electret effect during fragmentation of the rocks, (in russian), Izv. Acad.Sci. USSR, Fizika Zemli, 8, 104-108, 1983.

Balbachjan M.J., and Parhomenko E.I, Investigations ofinfluence of humidity on mechano-electret effect in the rocks, (in russian), Izv. Acad. Sci. USSR, Fizika Zemli, 12, 79-83, 1987.

Barri J. Globe-lightning and forked lightning, (in massian), Moscow, Mir, 1983.

Barsukov O.M., Skovorodkin J.P. Magnetic observation in the explosion region at Medeo, (in Mussian), News of AS USSR. The physics of the Earth. 1969. No. 5. p. 68-69.

Baum F.A.,Orlenko I.P.,Stanjukovich K.P., Chelyshev V.P., and Shehter B.I., Physics of explosion, (in russian), 704 pp., Moscow: Nauka, 1975.

Berdichevsky M.N. Electric prospect by the magneto-tellurium profiling, (in russian), Moscow, Nedra, 1968. Bevington P.R., Data reduction and error analysis for the physical sciences, 336 pp., N.Y.:McGraw-Hill, 1969.

Boev S.G., andGalanor A.N,. Charging of single crystalof LiF during cleaving, (in russian),PSB, 22, 10, 3069-3075, 1980.

Boronin A.P., Kapinos V.N., Mineev V.N. Physical mechanism of the electric field generation for explosions of the condensed explosives, (in missian), FGV, 1990, V. 26, 5 , pp. 110-116.

Boronin A.P., Kapinos V.N., Krener S.A., and Mineev V.N., On a physical mechanism of electromagnetic field generation by the explosion of condensed explosives charges, Literature review, (in mussian), The Physics of Combation and explosions, 26, 110-116, 1990 a.

Boronin A.P., Kapinos V.N., and Krenev S.A., On a physical mechanism of electromagnetic field generation by the explosion of condensed explosives charges, The results of the experimental studies, (in mussian), The Physics of Combation and explosions, 26, 117-123, $1990 \mathrm{~b}$.

Bricard J., Radiactivity and pollution influence on the electricity of the atmosphere, (in mussian), in book Atmospheric electricity problems, III International conference on electricity of the atmosphere and space, Leningrad, 
Gidrometeoizdat, 1969, pp. 68-104.

Brook M. et al. Artificial initiation of lighting discharges. J.G.R., vol.66, pp.3967-3969, 1961.

Cadastre of the rocks physical properties, mussian), Moscow, Nedra, 1975.

Caffin J.E., and Goodfellow T.L., Electrical effects associated with the mechanical deformation of single crystals of alkali halides , Nature, 176, 4488, 878-879, 1955.

Chalmers J.A., Atmospheric electricity, $2 d$ ed., 516 pp., Oxford, Pergamon press, 1967.

Chalmers J.A. Electrisity of the atmosphere, (in russian), Leningrad, Gidrometeoizdat, 1974.

Cherepanov G.P., About development of cracks in pressed bodies, (in mussian), PMM, 30, 1, 82-93.

Chernavsky E.A. Atmospheric electricity potential disturbances, (in mussian), Meteorologiya i gidrologiya $v$ Uzbekistane, Tashkent, FAN, 1955, $329 \mathrm{p}$.

Crawford D.A., and Schultz P.H., Laboratory observations of impact-generated magnetic fields, Nature, 336, 50-52, 1988.

Holzer R.E., Atmospheric electrical effects of nuclear explosions, J. Geophys. Res., 77, 5845-5855, 1972.

Davidson C., A study of recent earthquakes, London, 158, 1905.

Derjagin B.V., Krotova N.A., and Hrustalev J.A., Electrical properties of the surfaces, (in massian), Active surface of solid bodies, Moscow, 6-15,'1976.

Derjagin B.V., Krotova N.A., and Smilga V.G., Adhesionof solid bodies, (in russian), 279 pp. ,Moscow: Nauka, 1973.

Dorman I.I. Disturbance of magnetic field during the wave and stream motions in the conductive medium, (in russian), Magnetic hydrodynamics and dynamics of plasma, Riga, Latv. Pabl., 1962, p.63.

Eiby G.A. Earthquakes., New Zeland, Hinemann, 1980.

Eichelberger R.J., and Hauver G.E., Solid state transducers for recording of intense pressure pulses, In: Les ondes de detonation, Paris, 363-381, 1962.

Egorov P.V., Vasiljev O.B., Kormejchikov V.P., Kornejchikova N.M., and Nesterov N.N., Phenomen of the origin of solid charge in the rocks during theire mechanical 
stressing, (in russian), FTPRPI, 5, 101-104,1978.

Fnohovich A.S. Brief physical reference book, (in mussian) Moscow, 1976.

Frjanov J.S., Kurskeev A.K., Hasanbaev T.E., Bushuev A.V. Geomagnetic observation during MASSA experiment, (in russian) News of AS USSR. The physics of the Earth. 1985. No.11. p. 80-82.

Feynman R.P., Leighton R.B., Sands M. The Feynman Iectures on physics, V. 2, Addision-Wesler Publ. Company, Inc. Reading Massachusetts, Palo Alto, London, 1964.

Finkel V.M., The physical foundations of thedeceleration of fragmentation, (in mussian), 359 pp., Moscow:Metallurgia, 1977.

Finkel V.M., Golovin J.I., Sereda V.E., Kulikova G.P., and Zuev L.B., Electrical effects during the fragmentation of Lifcrystals in connection with the problem of control the crack, (in russian), PSB, 17, 3, 770-777, 1975.

Finkel V.M., Tjalin J.I., Golovin J.I., Muratova I.N., and Gorshener M.V., Electrification of alkali halides crystalsin spalling process, (in russian), PSB, 21,7 , 1943-1947, 1979.

Fishbach D.B., and Nowick A.C., Some transient electrical effects of plastic deformation in $\mathrm{NaCl}$ crystals, J.Phys.Chem.Solids, 5, 4, 302-315, 1958.

Fleman F., The Response of Magnetic Instruments to Earthquake waves., Journal of Geomagnetism and Geoelectricity, V. 18, 1966.

Frenkel Ya.I., Mechanism of the electrification of solid and liquid bodies during dispersion, (in russian), JETP, 18, 9,799-806, 1948.

Frencel Ya.I. Theory of the atmospheric electricity, (in russian), Moscow-Leningrad, GITTL, 1949.

Fux N.A. Mechanics of aerosols, (in russian), Moscow, AN USSR publish., 1955.

Gershenzon N.I., Zilpimiani D.O., Mandjgaladze P.V., Pohotelov O.A., and Chelidze Z.T., Electromagnetic emission ofcrack top during the fragmentation of ionic crystals, (in russian), Doklady AN SSSR, 288, 1, 75-78, 1986.

Gohberg M.B. et al. Electromagnetic radiation of rocks 
under the explosion loading, (in mussian), DAN USSR, 1987, V. 295, 2, pp. 321-325.

Goodenough J.B. A theory of main creation and coercive force in polycrystalline ferromagnetic, Phys. Rev., 1954, V. 95, Sr. II, No. 4, p. 917-932.

Gorbacher L.P., Surkov V.V. Disturbance of extermal magnetic field by the surface Rayleight wave, (in mussian), Magnitnaya gidrodinamika, 1987, J6 2, pp.3-12.

Gorskaya E.M., Scrynnikov R.G., Sokolov G.V. Variations of the magnetic field, induced by the sea-waves motion in shallow water, (in russian), Geomagnetizm \& aeronomia, 1972, 12, 수 1, 153-156.

Grigoriev V.G.,Nemirov A.S., and Sirotkin V.K., Structure of the shock waves in elastic-plastical relaxingmediums, (in russian), PMTF, 1, 153-160, 1979.

Gulielmi A.V. Generation of the electromagnetic field oscillations by the elastic waves in the conductive body, (in russian), Geomagnetizm \& aeronomia, 1986, V. XXVI, 佔 3, pp. 467-470.

Harris P., Mechanism for the shock polarization of dielectrics, J.Appl.Phys., 36, 5, Pt.1, 739-741, 1965.

Hasbrouk .P., Allen J.H. Quasi-static magnetic field changes associated with Cannikin nuclear explosions. Bull.Seism.Soc.Amer. 1972. V. 62. No. 6. P. 1479-1480.

Hoppel W.A. Theory of electrode effect. J.Atm.Terrest.Phys., 1967, v.29, No.4, p.709-721.

Imyanitor I.M. Devices and methods for atmospheric electricity development, (in mussian), Moscow, Gostechizdat, 1957, $483 \mathrm{p}$.

Imyanitor I.M., Devices and methods for studying atmospheric electricity, (in mssian), 1958.

Ivanov A.G., Lisitsyn J.V., and Novitskiy E.Z., Theproblem of polarization of dielectrics by the shock stressing, (in russian), JETP, 54, 1, 285-291, 1968.

Ivanov. A.G., and Novitskiy E.Z., Problem of double layerin presslyshocked dielectrics, (in mussian), PMTF, 5, 104-107, 1966.

Ivanov A.G., Mineev V.N., Novitsky E.Z., Janov V.A., and Bezrukov G.I., About anomalous polarization of sodium 
chloridecaused by the shock stressing, (in mussian), Letters to JETP, 2, 8, 353-356, 1965

Iranov B.A. and Basilevsky A.T., Estimation of maximum size and size-frequency distribution of fragments ejected from impact craters, (in missian), Abstracts of XIX all-Union Meteoritic Conference, Moscow, February 7-9, 1984, Vermadsky Institute, Moscow, p.115-117.

Jenkins G.M, and Watts D.G. Spectral analysis and its application. Holden-day, San Francisco, 1969.

Kamra A.K. Electrification in an Indian dust storm, Weather, 24, 145-146, 1969.

Kamra A.K., Measurement of the electrical properties of dust storms, J. Geophys. Res., 77, 5856-5869, 1972.

Kamra A.K., Experimental study of the electrification produced by the dispersion of dust into the air, J.Appl.Phys., 44, 125-131, 1973.

Kiljkeev R.S., and Jun R., Electrical effects associated with the deformation of the rocks, (in russian), FTPRPI, 2, 102-105, 1979 .

Kittel C., Introduction to physics of solid body, (in mussian), 791 pp., Moscow: Nauka, 1966.

Klein M.J., The structure of explosively shocked MgO crystals, Phil.Mag., 12, 18, 735-739, 1965.

KIein M.J., and Gager W.B., Generation of vacancies in MgO by deformation, J.Appl.Phys., 37, 11, 4112, 1966.

Klein M., Lonvet P., Morat P., Phys. Earth and Planet Inter., 1975, V. 10, 49.

Knopoff I.J., The interaction between elastic wave motions and a magnetic field in electrical conductors, J. Geophys. Res., 1955, V. 60, p. 441.

Kondorsky E.I. About gysterezis of ferromagnetics, (in mussian), JATF 1940. จ. 10. No. 4. p. 420-440.

Kondorsky E.I. To theory of stability of magnetic conditions in ferromagnetic materials on magnetization, (in mussian), JATF 1959. v. 37. No. 4(10). P. 1110-1115.

Kormfeljd M.I., About the origin of the surplus electrical charges in alkali halides crystals, (in missian), PSB, 12, 1, 318-319, 1970.

Kormfeljd M.I., Electricalcharges on the surface 
ofalkali halides crystals, (in mussian), PSB, 13, 2, 474-479, 1971.

Kornfeljd M.I., Electrification of ionic crystal byplastic deformation and splitting, (in musian), UFN, 116, $2,327-340,1975$.

Kozlov A.N., Fonarev G.A., Shumov L.A. Results of the observations of the sea-waves magnetic fields, (in russian), Geomagnetism \& Aeronomia, 1971, 11, 4, pp. 741-742.

Kozlov A.N., Pushkov A.N., Rahmatullin R.Sh., Skovorodkin J.P. Magnetic effects during explosions in rocks, (in russian), News of AS USSR. The physics of the Earth. 1974. No. 3. p. 66-71.

Krae A.P. Principles of geoelectrics, (in russian), p. 1, Moscow, 1951.

Krasnogorskaya N.V. Electricity of the atmospheric layers, nearest to the Earth and methods of its measurements, (in mussian), Leningrad, Gidrometizdat, 1972.

Krasnogorskaya N.V., Remizov V.P. Some results of the measurements of Earth's electric field variations. (in russian), DAN USSR, 1973, V. 212, t6 2, pp. 345-348.

\section{Krasnogorskaya N.V., MeInikov V.A., Rybin V.V.,}

Soloviev S.P., Methods of the electric field measurements, (in russian), in book Electric field in biosphere, Moscow, Nauka, 1984, v. 1.

Krasnogorskaya N.V., Soloviev S.P. Electric field of the Earth's atmosphere of IIF range, (in russian), In book Electromagnetic field in biosphere, Moscow, Nauka, 1984, V. 1.

Krotevich N.F., Semenov V.Yu., Fonarev G.A. Results of the experimental observations of the magnetic field from the sea-waves, (in russian), Geomagnetizm \& aeronomia, 1977, 17, N6 6, pp. 1088-1091.

Kuzmina N.V. et al. Seismic effect of the outcrop explosions in non-rock connected soils, (in mussian), Trudy IFZ AN USSR, 21 (188), 1962.

Landaw L.D., Lifshits E.M. Field theory, (in russian), Moscow, Fizmatgiz, 1960.

Landaw L.D., Lifshits E.M. Theory of elasticity, (in Mussian), Moscow, Nauka, 1965.

Landaw L.D., Lifshits E.M. Electrodynamics of continuous 
media, (in mussian), Moscow, Nauka, 1982.

Linde R.K.,Murri W.J., and Doran D.G. Shock-induced electrical polarization of alkali halides, J.Appl.Phys.,37, 7, 2527-2532, 1966.

Lisitsin J.V., Mineev V.N., and Novitsky E.Z., Someproblems of theory of polarizational transducer, (in russian), PMTF, 3, 56-60, 1970.

Loeb L.B., Static electrification, Springer-Verlag, Berlin, 1958.

Maclure K.C., Hafer R.A., Weaver J.T., Nature, 1964,204, 1290-1296.

Malkovich R.S., Socialized equations of diffusion in crystal, (in russian), FTT, 24, 2, 463-465,1982.

Mechanical effect of the underground explosion, (in russian), Moscow, Nedra, 1971.

Mineev V.N., and Ivanov A.G., Electromotive force caused by the shock pressing of the matter, (in russian), UFN, 119, 1, 75-109, 1976.

Mineev V.N., Ivanov A.G.,Lisitsin J.V.,Novitsky E.Z., andTjunjaev J.N., Electrical signals generated by the shock pressing of doped silicon, (in russian), JETP, 59, 10,1091-1102, 1970.

Mineer V.N., Ivanov A.G.,Novitsky E.Z.,Tjunjaev J.N. and Lisitsin J.V., Polarization of silicon and germanium by the shock stressing, (in russian), Letters to JETP , 5, 9,296-299, 1967.

Molotsky M.I., Dislocationalmechanism of the electrification of ionic crystals by splitting, (in russian), PSB, 18, 6, 1763-1764, 1976.

Molotskiy M.I., Ion-electronic mechanism of mechano-emission, (in russian), PSB, 19, 2, 642-644, 1977.

Molotsky M.I., Energy spectrum of mechanoelectrons, (in mussian), PSB, 25, 10, 2892-2895, 1983.

Morozov V.N. Physico-matematical modelling of the processes in the atmospheric layers, nearest to the Earth, (in russian), Trudy GGO, 1984, iss. 484, pp. 84-91.

Nabitovich I.D., Tsalj N.A., Shkribalo J.M., and Fechan V.T., Electrical charge on the surface of single crystal crack (in russian), Active surface of solid bodies,.Moscow: 16-21, 
1976.

Neilson F.W., Effects of strong shocks in ferroelectric materials, Bull.Amer.Phys.Soc., II, 2, 6, 302, 1957.

Neilson F.W., and Benedick W.B., Piezoelectric response of quarts beyond its Hugoniot elastic limit, Bull.Amer.Phys.Soc., II, 5, 7, 511, 1960.

Novatsky V. Electromagnetic effects in solids, (in russian), Nir, 1986.

Ogawa T., Analyses of measurement techniques of electric field and currents in the atmosphere, Contributions, Geophisical Institute, Kioto Uiversity, No 13, 111-137, 1973.

Parhomenko E.I., and Balbachjan M.J., Investigations ofeffect of electrification by friction in the rocks, (in russian), Izv. Acad. Sci. USSR, Fizika Zemli, 11, 100-107, 1981 .

Pierce C.B., Effect of pressure on the ionic conductivity in doped crystals of sodium chloride, potassium chloride and rubidium chloride, Phys.Rev., 123, 3, 744-754, 1961 .

Polivanov K.M. Ferromagnetics, (in mussian), Moscow-Leningrad, State energy Publishing House. 1957. 256 pages.

Pratt P.I. Point defects and mechanical properties of the ionic crystals. In: Vacincies and point defects, (in mussian), Moscow, Metallurgiya, 1961, $303 \mathrm{p}$.

Rodionov V.N., Adushkin. V.V., Kostjuchenko V.N., Nikolaevskiy V.N., Romashev A.N., and Tsvetkov V.M., Mechanical effect of underground burst, (in russian), 224 pp.,Moscow: Nedra, 1971.

Rulenko O.P., An electrification of the volcanic clouds. (in russian), Volcanology and seismology, 2, 71-83, 1985.

Rulenko O.P., Klimin N.N., Diakonova I.N., and Kirianov V.J., Study of the electrification of the cloud produced by the dispersion of volcanic ash, (in missian), Volcanology and seismology, 5, 17-29, 1986.

Semenov A.S. Electrical prospecting by natural electric field method, (in mussian), 200 pp., Moscow, Nedra, 1974.

Shapiro.A., Ivanov .A. The characteristics of dynamics magnetizement caused by strokes in natural ferromagnetics, (in russian), News of AS USSR. The physics of the Earth. 1969. No. 
5 p. 50-60.

Sheftel V.M., Chermyshev A.K. Atmospheric electricity reaction on isolated solar flash and flash series. (in mussian), Geomagnetism and aeronomia, 1992, v. 32,161 , pp. $111-117$.

Shertsov G.I., Migunov N.I., Sobolev G.A., and Kozlov E.V. , Electrification of feldspars by deformation and fragmentation, (in russian), Doklady AN SSSR, 225, 2, $313-315,1975$.

Shevtsova I.N. Charging of mobile dislocations and electrification of crystals under plastic deformation, (in russian), FTT, 25, 4, 1172-1179, 1983.

Sirotkin V.K., and Syrkov V.V., Mechanism of the origin of the solid charge under the shock pressing of ionic crystals, (in russian), PMTF, 4, 26-31, 1986.

Sobolev G.A., Demin V.A., Mechanical and electrical phenomena in the Earth, (in Mussian), Moscow, Nauka, 1980, $216 \mathrm{p}$.

Stacey F.D. The seismomagnetic effect. Pure and Appl. Geophys. 1964. V. 58. No.11. P. 5-23.

Stepanow A.W., Über den mechanismus der plastisen deformation, Zs.f.Phys, 61, 560-564, 1933.

Sweeney J.J. An investigation of the usefulness of extremely low-frequency electromagnetic measurements for treaty verification. Univ. Calif. Lawrence Livermore National Laboratory. Rept. UCRL-53899, 1989.

Syrkov V.V., Electromagnetic disturbances generated by underground burst, (in mussian), Theoretical questions of physics of explosions, Moscow, Energoatomizdat, 12-29, 1985.

Syrkov V.V., Electronic emission during the fragmentation of crystalline dielectrics, (in russian), JETP, $56,9,1818-1820,1986$.

Syrkov V.V., Eletromagnetic field generated by shock wave extending in condensed medium, (in russian), PMTF, 1, $29-36,1986$.

Syrkov V.V., Eletromagnetic effects of shock action onheterogeneous solid mediums, (in russian), Preprint MIFI 018-90, 20 pp., Moscow: MIFI, 1990.

Syrkov V.V., Eletromagnetic effects, produced by shock 
action on heterogeneous solid mediums, (in russian), Preprint MIFI, 32 pp., Moscow: MIFI, 1991.

Syrkov V.V., Electromagnetic field generated by the shockpressing and fragmentation of dielectrics and magneticmediums, (in russian), III Zababahinskie scientific readings, Reports, Cheljabinsk, 140-141, 1992.

Tamm I.E. The principles of the electricity theory, (in russian), Moscow, 1956.

Tammet H.F. Introduction to the linear theory of the specroscopy, (in russian), "Balgus", Tallinn, 1975.

Tjunjaev.J.N., and Mineev V.N., Characteristic property of polarization of LiF in shock waves, (in russian), FTT, 13, 2, 520-522, 1971.

Tjunjaev.J.N., and Mineev V.N., Mechanism for polarization of doped alkali halides crystals in shock waves, (in russian), FTT, 15, 5, 1901-1904, 1973.

Tjunjaev.J.N., Mineev V.N., and Ivanov A.G., and GutmansE.J., Influence of admixtures on polarization in $\mathrm{NaCl}$ crystalsin shock waves, (in russian), FTT, 11, 10, 3048-3050, 1969.

Tjunjaev.J.N., Umusovskaja A.A., Mineev V.N., and Ivanov A.G., Role of the dislocations in the shock polarization of alkalihalides crystals, (in russian), FTT, 10, 11, 3399-3405, 1968.

Tjapunina N.A., and Belozerova E.P., Charged dislocationsand characteristic of alkali halides crystals, (in mussian), UFN, 156, 4, 863-717,1988.

Tsal N.A., Vollbrandt I., Hrustalev J.A., Linke E., andDerjaginB.V., Generation of high energy electrons associated with fragmentation of solid bodies, (in russian), Doklady AN SSSR, 225, 2, 342-344, 1975.

Tsvetkov V.M., Sizov A.I., and Polikarpov A.D., About behaviour of brittle-failed medium during contained burst, (in Mussian), FTPRPI, 4, 36-42, 1977.

Tverskoy P.N. Atmospheric electricity, (in mussian), Leningrad, Gidrometeoizdat, 1949.

Uman M.A., Lightning, McGraw-Hill, New York, 1969.

Uman M.A., Natural and artificially initiated lightning and lightning test standards, Proceedings of the IEEE, vol. 76, 
N6 12, p.1548-1565, 1988.

Undzenkor B.A., Shapiro V.A. The seismomagnetic effect on the magnetite deposit. NewS of AS USSR. The physics of the Earth. 1967. No. 1. p. 121-126.

Urusovskaja A.A., Electrical effects of deformation in ionic crystals, (in russian), UFN, 96, 1, 39-60, 1968

Vorobjev A.A., Charging of surfaces associated with fragmentation, contact or friction of bodies, (in russian), 242 pp., Tomsk: VINITI, 1982.

Wait J.R., and Cambell L.I., The fields of an electric dipole in a semi-infinite conducting medium, J.Geophys.Res., $58 \mathrm{~A}, 1,21-28,1953$.

Wait J.R., The electromagnetic fields of a horizontal dipole in the presenseof a conducting half-space, Can.J.Phys., 39, 7, 1017-1028, 1961.

Warburton F., Caminiti R., The induced magnetic field of sea waves., J. Geophys. Res., 69, 4311-4318, 1964.

Weaver J.T., Magnetic Variations Associated with Ocean Waves and Swell., J. Geophys. Res., 70, J6 8, 1921-1929, 1965.

Willet J.C. An analysis of the electrode effect in the limit of strong turbulent mixing. J.G.R., 1978, No.C1,v.83 p. 402-408.

Williams E.R. et al. The role of electric space charge in nuclear lightning. Write in J.G.R. vol. 93, No.D2, p. 1679-1688. 1988.

Williams E.R. Storm clouds electrization, (in russian), $\mathrm{V}$ mire nauki, 1989, 佔 1, pp. 34-44.

Wong J.W.,Linde R.K., and White R.M., Electrical signals in dynamically stressed ionic crystals: A dislocation model, J.Appl.Phys., 40, 10, 4137-4145, 1969.

Yakushev V.V., Rozanov O.K., and Dremin A.N., Measurement of the relaxation time of polarization in shock wave, (in russian), JETP, 54, 2, 396-400, 1968.

Yerg D.G., and Johnson K.R., Short-period fluctuations in fair-weather electric field, J. Geophys. Res., 79, 2177-2184, 1974.

Zablocki C.J., Electrical transient observed during underground nuclear explosions, J.Geophys.Res., 71, $14,3523-3542,1966$. 
Zaborovsky A.I., Electroprospect, Moscow, 1963.

Zaidel R.M., Determination of the regime of the electricrelaxation caused by the shock stressing, (in mussian), JETP, 54, 4, 1253-1258, 1968.

Zakrevskiy V.A., Pahotin V.A., and Vaitkevich S.K., Electronic emission associated with the homo-axisal pressing stress of ionic crystals, (in missian), PSB, 21, 3, 723-729, 1979.

Zeljdovich J.B., Electromotive force caused by spread ofshock wave in dielectric, (in russian), JETP, 53, 1, 237-243, 1967.

Zybin K.Yu. et al., Vertical electric component of the geomagnetic field pulsations. (in russian), DAN USSR, 1974, V. 218 , N 4 .

*This work was performed under the auspices of the U.S. Department of Energy by Lawrence Livermore National Laboratory under contract No. W-7405-Eng-48. 

Technical Information Department . Lawrence Livermore National Laboratory University of California - Livermore, California 94551 